\title{
Z-Selective Cross-Metathesis and Homodimerization of 3E-1,3-Dienes: Reaction Optimization, Computational Analysis, and Synthetic Applications
}

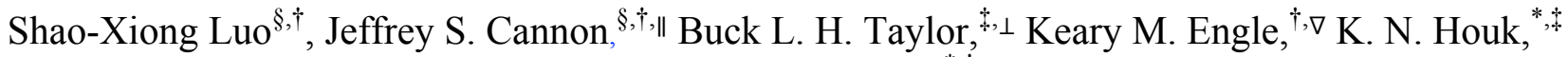 \\ and Robert H. Grubbs ${ }^{*} \dagger$ \\ $\dagger$ Arnold and Mabel Beckman Laboratories of Chemical Synthesis, California Institute of \\ Technology, Pasadena, CA 91125, United States \\ * Department of Chemistry and Biochemistry, University of California, Los Angeles, CA 90095 , \\ United States
}

\section{SUPPORTING INFORMATION}

\section{Table of Contents}

\section{Part 1: Experimental Data}

$\begin{array}{ll}\text { General experimental procedures } & \text { S2 }\end{array}$

General procedure for cross-metathesis between 3E-1,3-dienes and terminal olefins

Photographic guide for reaction setup

General Wittig olefination procedure for the synthesis of 3E-1,3-dienes

Characterization of new $3 E-1,3$-dienes

Characterization of new cross-metathesis products

Part 2: Computational Data

Computational details

Vinylcarbene conformations

Additional intermediates on diene-first Pathway A

Additional intermediates on alkene-first Pathway B

Reaction Pathway $\mathrm{C}$ starting from diastereomeric methylidene complex

Cartesian coordinates, energies, and thermal corrections for optimized structures

Part 3: References

References

Part 4: NMR Data

${ }^{1} \mathrm{H}$ and ${ }^{13} \mathrm{C}$ NMR spectra of new compounds 


\section{General experimental procedures}

All reactions were carried out in dry glassware under an argon atmosphere using standard Schlenk techniques or in a Vacuum Atmospheres Glovebox under a nitrogen atmosphere, unless otherwise specified. All solvents were purified by passage through solvent purification columns and further degassed by bubbling argon. NMR solvents were dried over $\mathrm{CaH}_{2}$ and vacuum transferred to a dry Schlenk flask and subsequently degassed with bubbling argon. $\mathrm{CDCl}_{3}$ was used as received. All $\alpha-$ olefins were filtered through a plug of neutral alumina prior to use. Ruthenium complexes $\mathbf{1}$ - $\mathbf{5}$ were obtained from Materia Inc. Ruthenium complex $\mathbf{6}^{1}$ and (2E)-7-(acetyloxy)-2-Heptenal ${ }^{2}$ were prepared according to their previously reported procedures. Other commercially available reagents and silica gel were used as received. Semi-preparative HPLC was performed using an Agilent 1200 series, a UV detector, and two ACE C18 columns (21.2mm x $250 \mathrm{~mm}, 5 \mu \mathrm{m})$ or an Agilent Eclipse XDB-C18 column $(9.4 \mathrm{~mm} \times 250 \mathrm{~mm}, 5 \mu \mathrm{m}) .{ }^{1} \mathrm{H}$ and ${ }^{13} \mathrm{C}$ NMR spectra were recorded on either a Bruker Ascend 400 spectrometer (400 MHz and $101 \mathrm{MHz}$, respectively) or a Varian Inova $500 \mathrm{MHz}$ (500 $\mathrm{MHz}$ and $126 \mathrm{MHz}$, respectively) and are internally referenced to $\mathrm{CDCl}_{3}$. High-resolution mass spectra (HRMS) were provided by the California Institute of Technology Mass Spectrometry Facility

using a JEOL JMS-600H High Resolution Mass Spectrometer. All HRMS were by positive-ion EI or FAB. 


\section{General procedure for cross-metathesis between $3 E$-1,3-dienes and terminal olefins:}

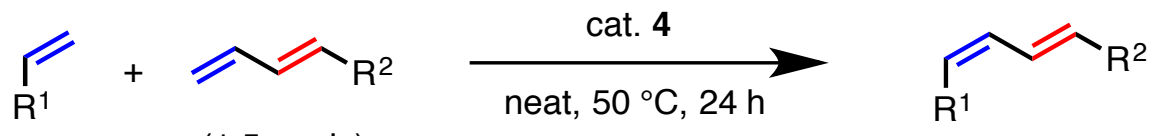

(1.5 equiv)

Scheme S1: General depiction of cross-metathesis between 3E-1,3-dienes and terminal olefins

In a nitrogen-filled glovebox, a $4 \mathrm{~mL}$ screw-top vial was charged with the appropriate terminal olefin $(0.50 \mathrm{mmol})$, the appropriate $3 E-1,3$-diene $(0.75 \mathrm{mmol})$, and catalyst $4(0.01-0.02 \mathrm{mmol})$. The vial was sealed with a septum cap, removed from the glovebox, wrapped at the interface with Teflon tape, and heated to $50{ }^{\circ} \mathrm{C}$. The reaction vessel was equipped with an argon inlet and a mineral oil bubbler outlet, both of which were connected via $18 \mathrm{G} \times 11 / 2$ " needles through the septum. The reaction mixture was kept under constant flow of argon (1-2 bubbles/s) for the duration of the reaction. The solution was maintained at $50{ }^{\circ} \mathrm{C}$ for $24 \mathrm{~h}$, cooled to ambient temperature and quenched with excess butyl or ethyl vinyl ether $(\sim 0.1 \mathrm{~mL})$. The solution was loaded onto a column loaded with silica gel. Elution first with hexanes $(50 \mathrm{~mL})$ to remove excess $3 E$-1,3-diene, followed by 20:1 hexanes:ethyl ether to elute the product, collected in a separate flask. The resulting solution was concentrated in vacuo to provide pure cross-metathesis product. 

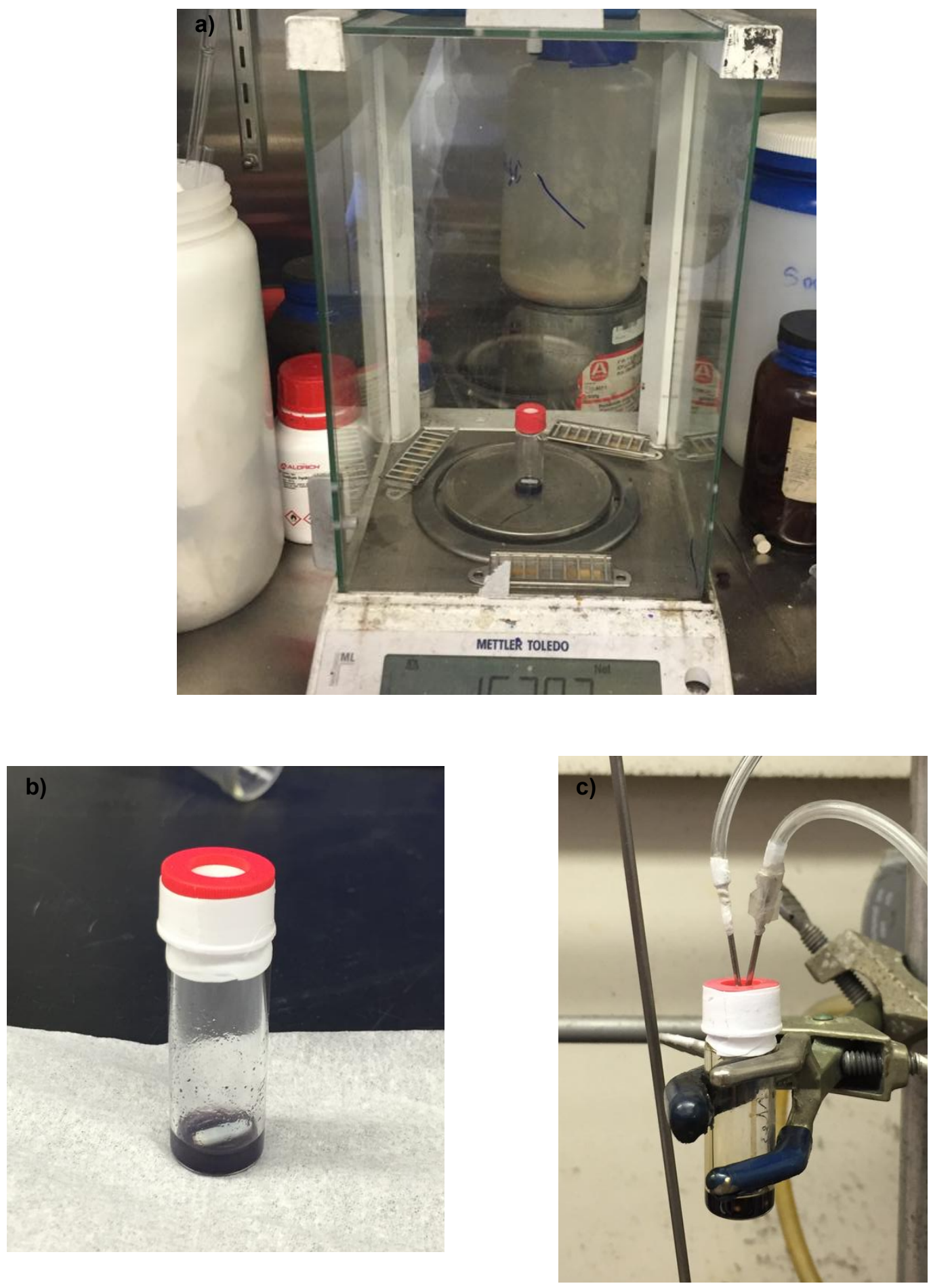

Figure S1: Photographic depiction of reaction setup following general procedure.

a) Weighing starting materials in glovebox. b) Sealing the vial with Teflon tape c) Connecting Ar inlet and outlet with needles 


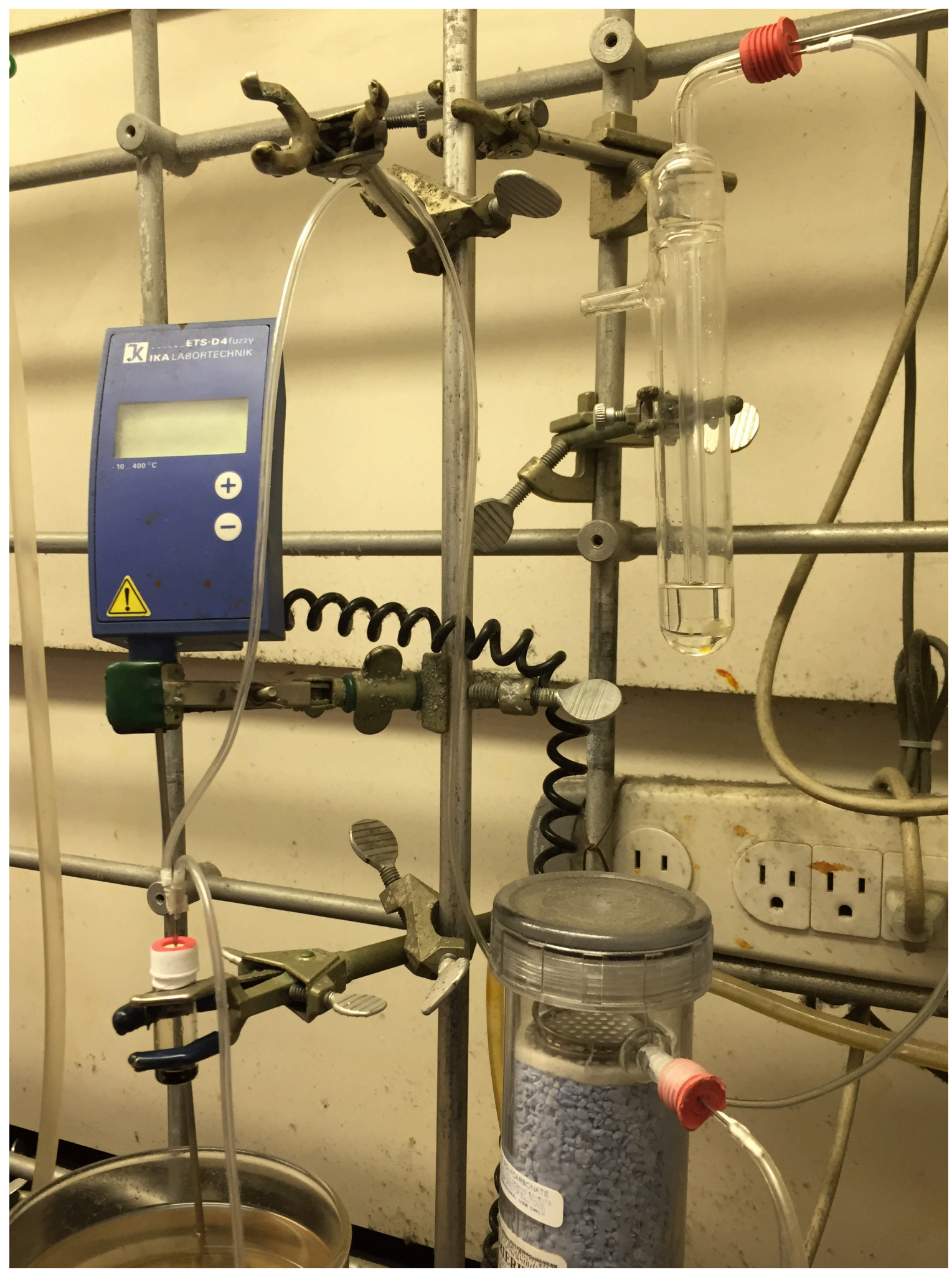

Figure S2: Photographic depiction of reaction setup following general procedure. 


\section{General Wittig olefination procedure for the synthesis of $3 E-1,3-d i e n e s:$}

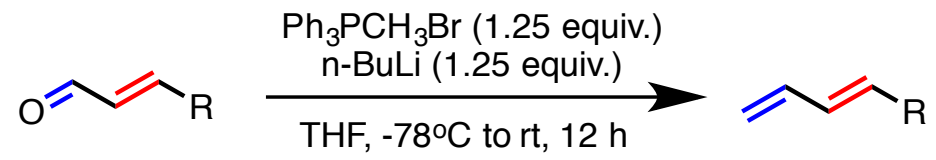

Scheme S2: General depiction of Wittig olefination for the synthesis of 3E-1,3-dienes

A flame-dried $100 \mathrm{~mL}$ Schlenk flask equipped with a Teflon-coated magnetic stir bar under Ar were charged with methyltriphenylphosphonium bromide (1.34 g, $3.75 \mathrm{mmol})$ and anhydrous THF (20 mL). $n$-Butyllithium solution $(2.5 \mathrm{M}$ in hexanes $)(1.5 \mathrm{~mL}, 3.75 \mathrm{mmol})$ was added at $0{ }^{\circ} \mathrm{C}$. The resulting yellow solution was allowed to warm to room temperature are stir until it became homogeneous (approximately $1 \mathrm{~h}$ ). The solution was cooled to $-78{ }^{\circ} \mathrm{C}$ in a dry ice/acetone bath, and the appropriate trans-enal was added $(3.0 \mathrm{mmol})$. The solution was allowed to warm to room temperature and stir overnight (approximately $12 \mathrm{~h}) \cdot \mathrm{Et}_{2} \mathrm{O}(30 \mathrm{~mL})$ was added, and the resulting heterogeneous solution was cooled to $-20{ }^{\circ} \mathrm{C}$ for $30 \mathrm{~min}$. The solution was filtered through a pad of Celite to remove the triphenylphosphine oxide precipitate, and the Celite was washed twice with $\mathrm{Et}_{2} \mathrm{O}$ that had been cooled to $0{ }^{\circ} \mathrm{C}$. The filtrate was concentrated in vacuo, and the resulting yellow oil was purified by silica gel column chromatography using a gradient solvent system $\left(100: 1\right.$ hexane: $\mathrm{Et}_{2} \mathrm{O} \rightarrow$ 10:1 hexane: $\left.\mathrm{Et}_{2} \mathrm{O}\right)$ as the eluent. The pure product was thus obtained as white solid or colorless oil. To prevent polymerization during prolonged storage, all 3E-1,3-dienes were kept under an $\mathrm{Ar}$ atmosphere at $-20^{\circ} \mathrm{C}$. 


\section{Characterization of $3 E-1,3$-dienes}

\section{(E)-tetradeca-1,3-diene (8a)}

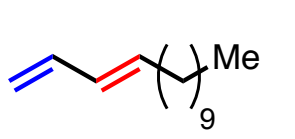

The title compound was prepared from $(E)$-tridec-2-enal $(1.96 \mathrm{~g}, 10.00 \mathrm{mmol})$ $8 \mathbf{a}$ according to the general Wittig procedure and was obtained as a colorless liquid (1.50 g, 77\% yield); Analytical data were in agreement with previously reported data. ${ }^{3}$

\section{(E)-4,8-dimethylnona-1,3,7-triene (8b)}

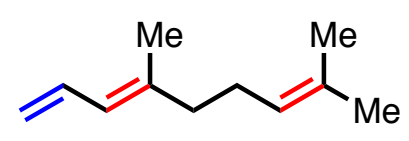

$8 b$

The title compound was prepared from (E)-3,7-dimethylocta-2,6-dienal (4.00 $\mathrm{g}, 26.30 \mathrm{mmol}$ ) according to the general Wittig procedure and was obtained as a colorless liquid (1.40 g, 35\% yield); Analytical data were in agreement with previously reported data. ${ }^{4}$

\section{(E)-octa-5,7-dien-1-yl acetate (8c)}

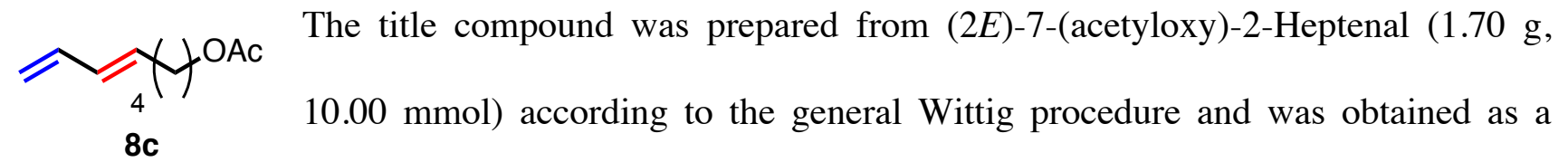
colorless liquid (927 mg, 55\% yield); ${ }^{1} \mathbf{H}$ NMR (400 MHz, Chloroform- $d$ ) $\delta 6.31$ (dt, $J=16.9,10.2$ $\mathrm{Hz}, 1 \mathrm{H}), 6.12-6.00(\mathrm{~m}, 1 \mathrm{H}), 5.68(\mathrm{dt}, J=14.6,7.0 \mathrm{~Hz}, 1 \mathrm{H}), 5.10(\mathrm{~d}, J=17.0 \mathrm{~Hz}, 1 \mathrm{H}), 4.97(\mathrm{~d}, J=$ $10.6 \mathrm{~Hz}, 1 \mathrm{H}), 4.09-4.03(\mathrm{~m}, 2 \mathrm{H}), 2.12(\mathrm{q}, J=6.5,5.8 \mathrm{~Hz}, 2 \mathrm{H}), 2.04(\mathrm{~d}, J=4.6 \mathrm{~Hz}, 3 \mathrm{H}), 1.68-1.60$ $(\mathrm{m}, 2 \mathrm{H}), 1.46(\mathrm{p}, J=7.1,6.6 \mathrm{~Hz}, 2 \mathrm{H}) ;{ }^{13} \mathbf{C}$ NMR $(101 \mathrm{MHz}$, Chloroform- $d) \delta$ 171.37, 137.27, 134.70, 131.56, 115.21, 64.52, 32.21, 28.24, 25.62, 21.17; HRMS (EI+) $m / z$ calcd for $\mathrm{C}_{10} \mathrm{H}_{16} \mathrm{O}\left[\mathrm{M}^{+}\right]$ 168.1150, found 168.1140. 


\section{(E)-1-(buta-1,3-dien-1-yl)-4-methoxybenzene (8e)}

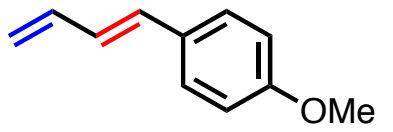

$8 e$

The title compound was prepared from trans-4-Methoxy-cinnamaldehyde

(972 $\mathrm{mg}, 6.00 \mathrm{mmol}$ ) according to the general Wittig procedure and was obtained as a white solid (815 mg, 81\% yield); ${ }^{1} \mathbf{H}$ NMR $(400 \mathrm{MHz}$, Chloroform- $d) \delta 7.39-7.32(\mathrm{~m}$, 2H), $6.90-6.84(\mathrm{~m}, 2 \mathrm{H}), 6.72-6.63(\mathrm{~m}, 1 \mathrm{H}), 6.56-6.43(\mathrm{~m}, 2 \mathrm{H}), 5.33-5.25(\mathrm{~m}, 1 \mathrm{H}), 5.15-5.10$ (m, 1H), $3.82(\mathrm{~s}, 3 \mathrm{H}) ;{ }^{13} \mathbf{C}$ NMR (101 MHz, Chloroform-d) $\delta 159.39,137.49,132.52,130.04,127.77$, 116.59, 114.19, 55.43; HRMS (FAB+) $m / z$ calcd for $\mathrm{C}_{11} \mathrm{H}_{12} \mathrm{O}[\mathrm{M}]^{+} 168.0888$, found 168.0887 .

\section{(E)-octa-5,7-dien-1-ol (8f)}

To a $500 \mathrm{~mL}$ round-bottom flask equipped with a Teflon-coated magnetic stir bar 8 were added (E)-octa-5,7-dien-1-yl acetate $(\mathbf{8 c})(252 \mathrm{mg}, 1.50 \mathrm{mmol})$, sodium hydroxide $(60.0 \mathrm{mg}, 1.50 \mathrm{mmol}), \mathrm{H}_{2} \mathrm{O}(50 \mathrm{~mL})$, and $\mathrm{MeOH}(50 \mathrm{~mL})$. The reaction mixture was stirred at $60{ }^{\circ} \mathrm{C}$ for $5 \mathrm{~h}$. The flask was allowed to cool to room temperature, and the reaction was extracted with ethyl acetate $(3 \times 150 \mathrm{~mL})$. The combined organic layers were washed with water $(100$ $\mathrm{mL})$ and brine $(2 \times 100 \mathrm{~mL})$, dried over $\mathrm{Na}_{2} \mathrm{SO}_{4}$, filtered, and concentrated in vacuo. Purification by silica gel column chromatography using a gradient solvent system (4:1 hexane:EtOAc $\rightarrow$ 1:1 hexane:EtOAc) as the eluent provided product as colorless liquid (157 mg, 82\% yield); ${ }^{1} \mathbf{H}$ NMR (400 MHz, Chloroform- $d) \delta 6.30(\mathrm{dt}, J=17.0,10.3 \mathrm{~Hz}, 1 \mathrm{H}), 6.13-6.02(\mathrm{~m}, 1 \mathrm{H}), 5.70(\mathrm{dt}, J=14.7,6.9$ $\mathrm{Hz}, 1 \mathrm{H}), 5.15-5.05(\mathrm{~m}, 1 \mathrm{H}), 5.01-4.93(\mathrm{~m}, 1 \mathrm{H}), 3.65(\mathrm{t}, J=6.5 \mathrm{~Hz}, 2 \mathrm{H}), 2.20-2.06(\mathrm{~m}, 2 \mathrm{H}), 1.65$ - $1.55(\mathrm{~m}, 2 \mathrm{H}), 1.48(\mathrm{dtd}, J=11.0,8.4,7.8,5.6 \mathrm{~Hz}, 2 \mathrm{H}) ;{ }^{13} \mathrm{C}$ NMR $(101 \mathrm{MHz}$, Chloroform- $d) \delta$ 137.33, 135.03, 131.41, 115.07, 62.96, 32.37, 25.43; HRMS (FAB+) m/z calcd for $\mathrm{C}_{8} \mathrm{H}_{15} \mathrm{O}[\mathrm{M}]^{+}$ 127.1123, found 127.1122. 


\section{Characterization of cross-metathesis products between $3 E$-1,3-dienes and terminal olefins}

(8Z,10E)-henicosa-8,10-dien-1-ol (9a)

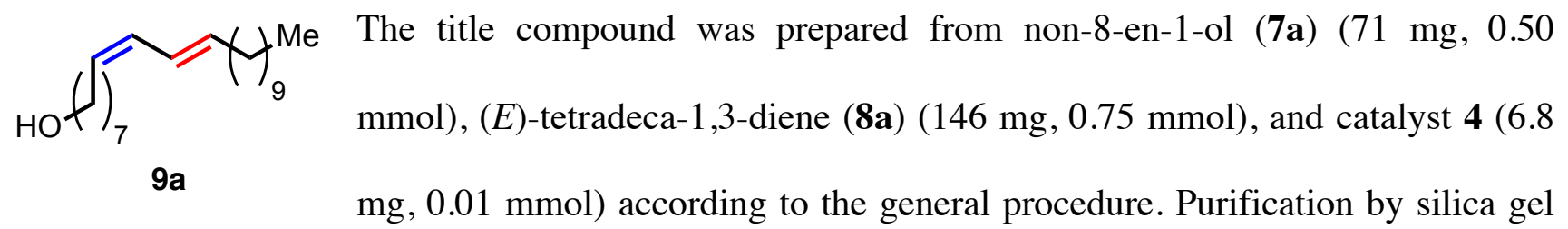

column chromatography using pure hexane (to elute unreacted $(E)$-tetradeca-1,3-diene $(\mathbf{8 a})$ ) followed by 10:1 hexane:Et ${ }_{2} \mathrm{O}$ provided the product as a colorless oil (130 mg, 84\% yield). ${ }^{1} \mathbf{H}$ NMR (500 MHz, Chloroform- $d) \delta 6.34-6.22(\mathrm{~m}, 2 \mathrm{H}), 5.93(\mathrm{t}, J=10.9 \mathrm{~Hz}, 1 \mathrm{H}), 5.64(\mathrm{dt}, J=14.6,7.0 \mathrm{~Hz}, 1 \mathrm{H})$, $5.28(\mathrm{dt}, J=10.7,7.6 \mathrm{~Hz}, 1 \mathrm{H}), 3.61(\mathrm{t}, J=6.7 \mathrm{~Hz}, 2 \mathrm{H}), 2.14(\mathrm{q}, J=6.8 \mathrm{~Hz}, 2 \mathrm{H}), 2.08(\mathrm{q}, J=7.1 \mathrm{~Hz}$, 2H), $1.55(\mathrm{p}, J=6.7 \mathrm{~Hz}, 2 \mathrm{H}), 1.40-1.24(\mathrm{~m}, 24 \mathrm{H}), 0.87(\mathrm{t}, J=6.9 \mathrm{~Hz}, 3 \mathrm{H}) ;{ }^{13} \mathbf{C} \mathbf{N M R}(126 \mathrm{MHz}$, Chloroform-d) $\delta 134.84,129.98,128.79,125.66,63.04,33.01,32.86,32.03,29.77,29.75,29.64$, 29.55, 29.46, 29.42, 29.38, 29.3, 27.77, 25.82, 22.80, 14.22; HRMS (FAB+) $\mathrm{m} / z$ Calcd for $\mathrm{C}_{21} \mathrm{H}_{40} \mathrm{O}$ $[\mathrm{M}]^{+}$308.3079, found 308.3077.

((E)-3-(((2Z,4E)-pentadeca-2,4-dien-1-yl)oxy)prop-1-en-1-yl)benzene (9b)

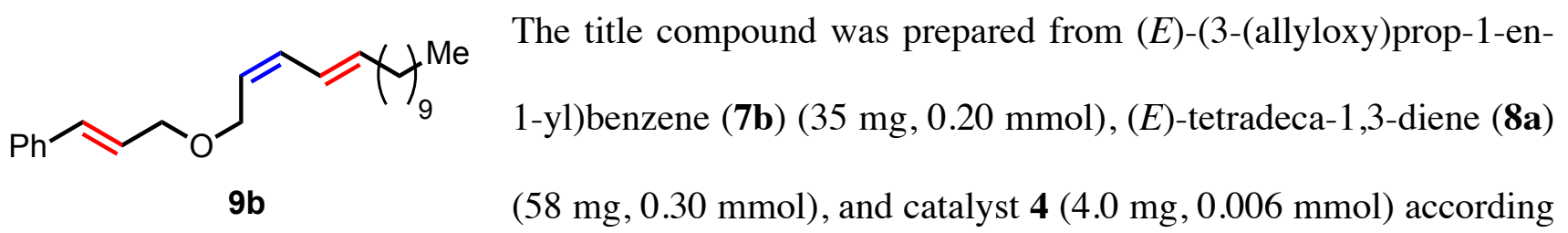

to the general procedure. Purification by silica gel column chromatography using pure hexane (to elute unreacted $(E)$-tetradeca-1,3-diene (8a)) followed by 20:1 hexane: $\mathrm{Et}_{2} \mathrm{O}$ provided the product as a colorless oil (56 mg, 82\% yield). ${ }^{1} \mathbf{H}$ NMR (500 MHz, Chloroform- $\left.d\right) \delta 7.43-7.39$ (m, 2H), 7.33 (td, $J=6.9,6.4,1.7 \mathrm{~Hz}, 2 \mathrm{H}), 7.28-7.24(\mathrm{~m}, 1 \mathrm{H}), 6.63(\mathrm{dd}, J=15.9,5.6 \mathrm{~Hz}, 1 \mathrm{H}), 6.36-6.29(\mathrm{~m}, 2 \mathrm{H})$, 
$6.16(\mathrm{t}, J=11.2 \mathrm{~Hz}, 1 \mathrm{H}), 5.78(\mathrm{dt}, J=14.5,7.0 \mathrm{~Hz}, 1 \mathrm{H}), 5.49(\mathrm{dt}, J=11.0,6.9 \mathrm{~Hz}, 1 \mathrm{H}), 4.22(\mathrm{dd}, J=$ $6.9,1.3 \mathrm{~Hz}, 2 \mathrm{H}), 4.18(\mathrm{dd}, J=6.1,1.4 \mathrm{~Hz}, 2 \mathrm{H}), 2.11(\mathrm{q}, J=7.3 \mathrm{~Hz}, 2 \mathrm{H}), 1.43-1.37(\mathrm{~m}, 2 \mathrm{H}), 1.28(\mathrm{~s}$, 14H), $0.91(\mathrm{t}, J=7.0 \mathrm{~Hz}, 3 \mathrm{H}) ;{ }^{13} \mathrm{C}$ NMR (126 MHz, Chloroform- $d$ ) $\delta 137.57,136.86,132.69$, $132.20,128.65,127.76,126.61,126.27,125.15,124.83,70.66,65.87,33.02,32.05,29.76,29.75$, 29.64, 29.48, 29.40, 29.36, 22.83, 14.27; HRMS (EI+) $\mathrm{m} / \mathrm{z}$ calcd for $\mathrm{C}_{24} \mathrm{H}_{36} \mathrm{O}[\mathrm{M}]^{+} 340.2766$, found 340.2768 .

\section{tert-butyl ((2Z,4E)-pentadeca-2,4-dien-1-yl)carbamate (9c)}

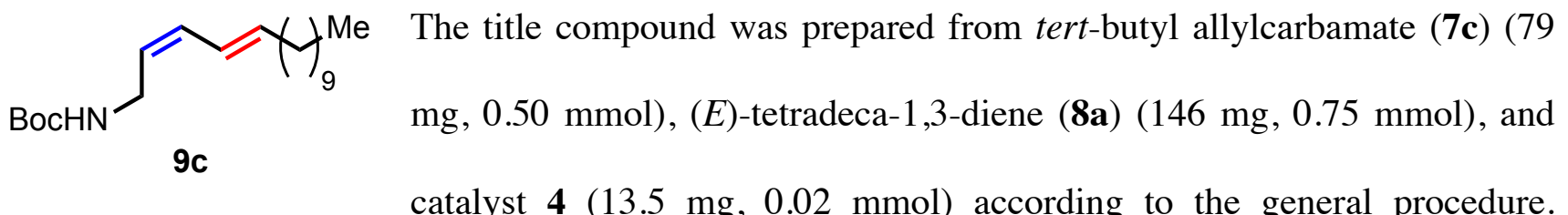

Purification by silica gel column chromatography using pure hexane (to elute unreacted $(E)$ tetradeca-1,3-diene (8a)) followed by 20:1 hexane:Et ${ }_{2} \mathrm{O}$ provided the product as a colorless oil (97 mg, 60\% yield). ${ }^{1}$ H NMR (400 MHz, Chloroform- $d$ ) $\delta 6.35-6.21(\mathrm{~m}, 1 \mathrm{H}), 6.04(\mathrm{t}, J=10.9 \mathrm{~Hz}, 1 \mathrm{H})$, $5.74(\mathrm{dt}, J=14.6,7.0 \mathrm{~Hz}, 1 \mathrm{H}), 5.29(\mathrm{dt}, J=10.5,7.2 \mathrm{~Hz}, 1 \mathrm{H}), 4.49(\mathrm{~s}, 1 \mathrm{H}), 3.87(\mathrm{~s}, 2 \mathrm{H}), 2.09(\mathrm{q}, J=$ $7.0 \mathrm{~Hz}, 2 \mathrm{H}), 1.45(\mathrm{~s}, 9 \mathrm{H}), 1.38(\mathrm{t}, J=7.1 \mathrm{~Hz}, 2 \mathrm{H}), 1.26(\mathrm{~s}, 14 \mathrm{H}), 0.88(\mathrm{t}, J=6.8 \mathrm{~Hz}, 3 \mathrm{H}) ;{ }^{13} \mathbf{C} \mathbf{N M R}$ (101 MHz, Chloroform-d) $\delta$ 155.94, 137.58, 131.57, 124.78, 124.73, 38.06, 33.03, 32.07, 29.78, 29.76, 29.66, 29.50, 29.41, 29.37, 28.57, 22.85, 14.27; HRMS (FAB+) m/z Calcd for $\mathrm{C}_{20} \mathrm{H}_{36} \mathrm{NO}_{2}$ $\left[(\mathrm{M}+\mathrm{H})-\mathrm{H}_{2}\right]^{+}$322.2746, found 322.2772. 
$(10 Z, 12 E)$-tricosa-10,12-dienal (9d)

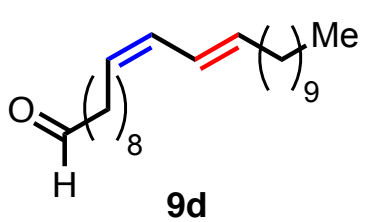

The title compound was prepared from undec-10-enal (7d) $(84 \mathrm{mg}, 0.50$ mmol), (E)-tetradeca-1,3-diene (8a) $(146 \mathrm{mg}, 0.75 \mathrm{mmol})$, and catalyst 4 (13.5 $\mathrm{mg}, 0.02 \mathrm{mmol}$ ) according to the general procedure. Purification by

silica gel column chromatography using pure hexane (to elute unreacted $(E)$-tetradeca-1,3-diene $(\mathbf{8 a})$ ) followed by 20:1 hexane:Et ${ }_{2} \mathrm{O}$ provided the product as a colorless oil (99 $\mathrm{mg}, 59 \%$ yield). ${ }^{1} \mathbf{H}$ NMR $(400 \mathrm{MHz}$, Chloroform- $d) \delta 9.76(\mathrm{t}, J=1.9 \mathrm{~Hz}, 1 \mathrm{H}), 6.35-6.23(\mathrm{~m}, 1 \mathrm{H}), 5.94(\mathrm{t}, J=11.1 \mathrm{~Hz}, 1 \mathrm{H})$, $5.65(\mathrm{dt}, J=14.6,7.0 \mathrm{~Hz}, 1 \mathrm{H}), 5.29(\mathrm{dt}, J=10.7,7.6 \mathrm{~Hz}, 1 \mathrm{H}), 2.41(\mathrm{td}, J=7.4,1.9 \mathrm{~Hz}, 2 \mathrm{H}), 2.15(\mathrm{q}$, $J=6.6 \mathrm{~Hz}, 2 \mathrm{H}), 2.09(\mathrm{q}, J=7.4 \mathrm{~Hz}, 2 \mathrm{H}), 1.62(\mathrm{p}, J=7.3 \mathrm{~Hz}, 2 \mathrm{H}), 1.37-1.24(\mathrm{~m}, 26 \mathrm{H}), 0.91-0.85$ (m, 3H); ${ }^{13} \mathbf{C}$ NMR (101 MHz, Chloroform-d) $\delta$ 203.03, 134.92, 130.09, 128.82, 125.71, 44.07, $33.06,32.07,29.85,29.79,29.69,29.59,29.50,29.46,29.45,29.42,29.32,29.31,27.82,22.85$, 22.24, 14.27; HRMS (EI+) $m / z$, Calcd for $\mathrm{C}_{23} \mathrm{H}_{42} \mathrm{O}[\mathrm{M}]^{+} 334.3236$, found 334.3246.

\section{4,4,5,5-tetramethyl-2-((2Z,4E)-pentadeca-2,4-dien-1-yl)-1,3,2-dioxaborolane (9e)}

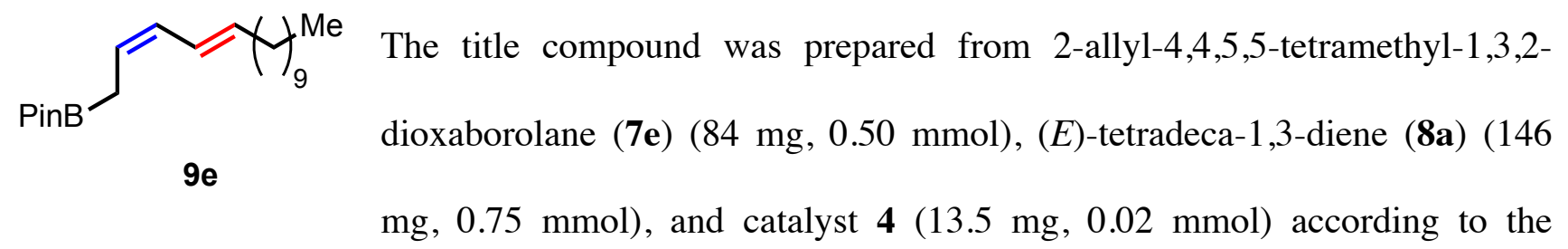
general procedure. ${ }^{1} \mathrm{H}$ NMR yield (60\%) was determined by characteristic olefin chemical shifts $(5.4$ - $6.3 \mathrm{ppm})$ with reference to nitrobenzene internal standard $(61.5 \mathrm{mg}, 0.50 \mathrm{mmol})$. Attempts were made to purify this product via silica gel chromatography, but were proved to be unsuccessful due to the instability of the compound on silica gel and its tendency to co-elute with the Hoveyda styrene generated in situ upon catalyst activation. In the interest of completeness, below we have included ${ }^{1} \mathrm{H}$ NMR spectra of the crude reaction mixture and of a $\sim 94 \%$ pure sample of the product. ${ }^{1} \mathbf{H}$ NMR (400 
MHz, Chloroform- $d) \delta 6.34-6.25(\mathrm{~m}, 1 \mathrm{H}), 5.96(\mathrm{t}, J=10.9 \mathrm{~Hz}, 1 \mathrm{H}), 5.64(\mathrm{dt}, J=14.6,7.0 \mathrm{~Hz}, 1 \mathrm{H})$, $5.47-5.38(\mathrm{~m}, 1 \mathrm{H}), 2.08(\mathrm{q}, J=7.0 \mathrm{~Hz}, 2 \mathrm{H}), 1.81(\mathrm{~d}, J=8.1 \mathrm{~Hz}, 2 \mathrm{H}), 1.41-1.37(\mathrm{~m}, 2 \mathrm{H}), 1.28(\mathrm{~d}, J$ $=15.5 \mathrm{~Hz}, 14 \mathrm{H}), 1.25(\mathrm{~s}, 12 \mathrm{H}), 0.88(\mathrm{t}, J=6.8 \mathrm{~Hz}, 3 \mathrm{H}) . \mathbf{H R M S}(\mathrm{FAB}+) \mathrm{m} / z$ Calcd for $\mathrm{C}_{21} \mathrm{H}_{39} \mathrm{BO}_{2}$ $[\mathrm{M}]^{+}$334.3043, found 334.3041.

\section{(5Z,7E)-octadeca-5,7-dien-1-yl acetate (9f)}

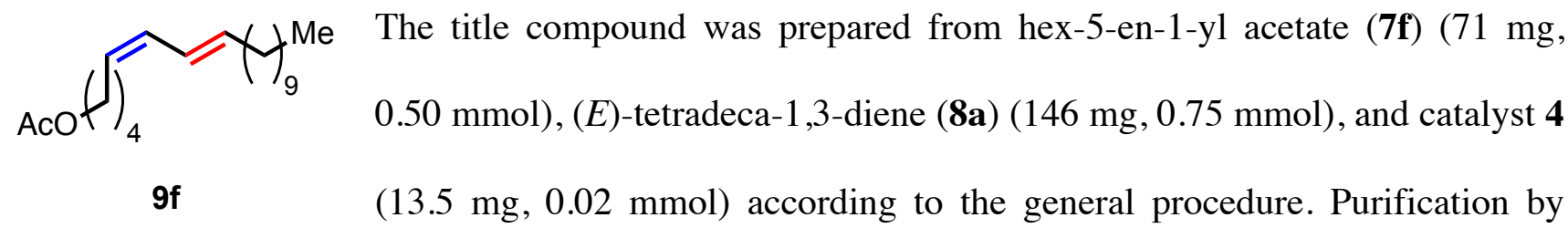

silica gel column chromatography using pure hexane (to elute unreacted $(E)$-tetradeca-1,3-diene $(\mathbf{8 a})$ ) followed by 20:1 hexane: $\mathrm{Et}_{2} \mathrm{O}$ provided the product as a colorless oil (107 $\mathrm{mg}, 69 \%$ yield). ${ }^{1} \mathbf{H}$ NMR (400 MHz, Chloroform- $d$ ) $\delta 6.34-6.21(\mathrm{~m}, 1 \mathrm{H}), 5.96(\mathrm{t}, J=11.0 \mathrm{~Hz}, 1 \mathrm{H}), 5.67(\mathrm{dt}, J=14.6,7.0 \mathrm{~Hz}$, 1H), $5.27(\mathrm{dt}, J=10.8,7.6 \mathrm{~Hz}, 1 \mathrm{H}), 4.06(\mathrm{t}, J=6.7 \mathrm{~Hz}, 2 \mathrm{H}), 2.19(\mathrm{qd}, J=7.5,1.3 \mathrm{~Hz}, 2 \mathrm{H}), 2.09(\mathrm{q}, J$ $=7.3 \mathrm{~Hz}, 2 \mathrm{H}), 2.04(\mathrm{~s}, 3 \mathrm{H}), 1.69-1.61(\mathrm{~m}, 2 \mathrm{H}), 1.49-1.42(\mathrm{~m}, 2 \mathrm{H}), 1.39-1.23(\mathrm{~m}, 16 \mathrm{H}), 0.93-$ $0.82(\mathrm{~m}, 3 \mathrm{H}) ;{ }^{13} \mathbf{C}$ NMR $(101 \mathrm{MHz}$, Chloroform-d) $\delta 171.31,135.37,129.37,129.11,125.51,64.57$, $33.05,32.06,29.78,29.77,29.67,29.55,29.49,29.41,28.32,27.36,26.17,22.84,21.14,14.26$; HRMS (EI+) $m / z$ Calcd for $\mathrm{C}_{20} \mathrm{H}_{36} \mathrm{O}_{2}[\mathrm{M}]^{+}$308.2715, found 308.2713.

\section{(5Z,7E)-octadeca-5,7-dien-2-one (9g)}

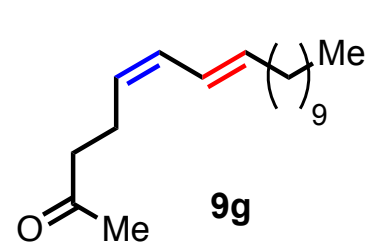

The title compound was prepared from hex-5-en-2-one (7g) (49 $\mathrm{mg}, 0.50$ mmol), (E)-tetradeca-1,3-diene (8a) (146 mg, $0.75 \mathrm{mmol})$, and catalyst 4 (13.5 $\mathrm{mg}, 0.02 \mathrm{mmol}$ ) according to the general procedure. Purification by silica gel column chromatography using pure hexane (to elute unreacted $(E)$-tetradeca-1,3-diene $(\mathbf{8 a})$ ) followed by 20:1 hexane:Et ${ }_{2} \mathrm{O}$ provided the product as a colorless oil (55 mg, $41 \%$ yield). ${ }^{1} \mathbf{H}$ NMR 
$(400 \mathrm{MHz}$, Chloroform- $d$ ) $\delta 6.29$ (ddq, $J=14.9,11.0,1.3 \mathrm{~Hz}, 1 \mathrm{H}), 5.96(\mathrm{t}, J=11.1 \mathrm{~Hz}, 1 \mathrm{H}), 5.68(\mathrm{dt}$, $J=14.6,7.0 \mathrm{~Hz}, 1 \mathrm{H}), 5.23(\mathrm{dt}, J=10.7,7.3 \mathrm{~Hz}, 1 \mathrm{H}), 2.54-2.48(\mathrm{~m}, 2 \mathrm{H}), 2.47-2.39$ (m, 2H), 2.14 (s, 3H), 2.09 (q, $J=6.9 \mathrm{~Hz}, 2 \mathrm{H}), 1.40-1.35(\mathrm{~m}, 2 \mathrm{H}), 1.26(\mathrm{~s}, 14 \mathrm{H}), 0.91-0.84(\mathrm{~m}, 3 \mathrm{H}) ;{ }^{13} \mathbf{C} \mathbf{N M R}$ (101 MHz, Chloroform- $d$ ) $\delta$ 208.42, 135.96, 129.89, 127.42, 125.23, 43.68, 33.04, 32.06, 30.09, 29.77, 29.77, 29.66, 29.49, 29.41, 22.84, 22.22, 14.26; HRMS (EI+) $\mathrm{m} / \mathrm{z}$ Calcd for $\mathrm{C}_{18} \mathrm{H}_{32} \mathrm{O}[\mathrm{M}]^{+}$ 264.2453, found 264.2442.

\section{Methyl-((2Z,4E)-pentadeca-2,4-dien-1-yl) carbonate (9h)}

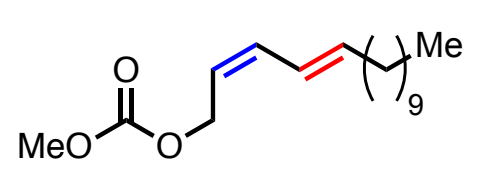

$9 \mathrm{~h}$

The title compound was prepared from allyl methyl carbonate (7h) $(58$ $\mathrm{mg}, 0.50 \mathrm{mmol}),(E)$-tetradeca-1,3-diene (8a) $(146 \mathrm{mg}, 0.75 \mathrm{mmol})$, and catalyst $4(13.5 \mathrm{mg}, 0.02 \mathrm{mmol})$ according to the general procedure.

Purification by silica gel column chromatography using pure hexane (to elute unreacted $(E)$ tetradeca-1,3-diene (8a)) followed by 20:1 hexane: $\mathrm{Et}_{2} \mathrm{O}$ provided the product as a colorless oil (60 $\mathrm{mg}, 42 \%$ yield). ${ }^{1} \mathbf{H}$ NMR (400 MHz, Chloroform- $d$ ) $\delta 6.35-6.27(\mathrm{~m}, 1 \mathrm{H}), 6.17(\mathrm{t}, J=11.1 \mathrm{~Hz}, 1 \mathrm{H})$, $5.80(\mathrm{dt}, J=14.5,7.0 \mathrm{~Hz}, 1 \mathrm{H}), 5.43(\mathrm{dt}, J=10.8,7.2 \mathrm{~Hz}, 1 \mathrm{H}), 4.79(\mathrm{dd}, J=7.2,1.1 \mathrm{~Hz}, 2 \mathrm{H}), 3.78(\mathrm{~s}$, 3H), $2.11(\mathrm{q}, J=7.0 \mathrm{~Hz}, 2 \mathrm{H}), 1.42-1.37(\mathrm{~m}, 2 \mathrm{H}), 1.26(\mathrm{~s}, 14 \mathrm{H}), 0.91-0.85(\mathrm{~m}, 3 \mathrm{H}),{ }^{13} \mathbf{C}$ NMR $(101$ MHz, Chloroform- $d$ ) $\delta$ 155.94, 139.07, 133.96, 124.57, 121.00, 64.00, 54.91, 33.03, 32.06, 29.76, 29.74, 29.64, 29.49, 29.39, 29.25, 22.84, 14.27; HRMS (FAB+) $\mathrm{m} / z$ Calcd for $\mathrm{C}_{17} \mathrm{H}_{29} \mathrm{O}_{3}\left[(\mathrm{M}+\mathrm{H})-\mathrm{H}_{2}\right]^{+}$ 281.2117 , found 281.2135 . 
(7Z,9E)-1-bromoicosa-7,9-diene (9i)

Br $\int_{6} \int_{9 \mathbf{i}}^{\mathrm{Me}}$ The title compound was prepared from 8-bromooct-1-ene (7i) (31 $\mathrm{mg}, 0.17$ $\mathrm{mg}, 0.007 \mathrm{mmol}$ ) according to the general procedure. Purification by silica gel

column chromatography using pure hexane (to elute unreacted $(E)$-tetradeca-1,3-diene $(\mathbf{8 a})$ ) followed by 20:1 hexane: $\mathrm{Et}_{2} \mathrm{O}$ provided the product as a colorless oil $\left(20 \mathrm{mg}, 34 \%\right.$ yield). ${ }^{1} \mathbf{H}$ NMR (400 MHz, Chloroform- $d) \delta 6.34-6.23(\mathrm{~m}, 1 \mathrm{H}), 5.95(\mathrm{t}, J=10.9 \mathrm{~Hz}, 1 \mathrm{H}), 5.66(\mathrm{dt}, J=14.5,7.0 \mathrm{~Hz}, 1 \mathrm{H}), 5.28$ $(\mathrm{dt}, J=10.7,7.6 \mathrm{~Hz}, 1 \mathrm{H}), 3.41(\mathrm{t}, J=6.8 \mathrm{~Hz}, 2 \mathrm{H}), 2.16(\mathrm{q}, J=7.1 \mathrm{~Hz}, 2 \mathrm{H}), 2.09$ (q, $J=7.1 \mathrm{~Hz}, 2 \mathrm{H})$, $1.86(\mathrm{p}, J=6.9 \mathrm{~Hz}, 2 \mathrm{H}), 1.48-1.33(\mathrm{~m}, 8 \mathrm{H}), 1.26(\mathrm{~s}, 14 \mathrm{H}), 0.88(\mathrm{t}, J=6.8 \mathrm{~Hz}, 3 \mathrm{H}) ;{ }^{13} \mathbf{C ~ N M R}(101$ $\mathrm{MHz}$, Chloroform-d) $\delta 135.11,129.77,128.99,125.61,34.16,33.06,32.92,32.07,29.78,29.68$, 29.63, 29.57, 29.50, 29.42, 28.48, 28.19, 27.67, 22.85, 14.29; HRMS (FAB+) $m / z$ Calcd for $\mathrm{C}_{20} \mathrm{H}_{37} \mathrm{Br}$ $[\mathrm{M}]^{+}$356.2079, found 356.2068.

(4Z,6E)-heptadeca-4,6-dienenitrile $(9 \mathbf{j})$

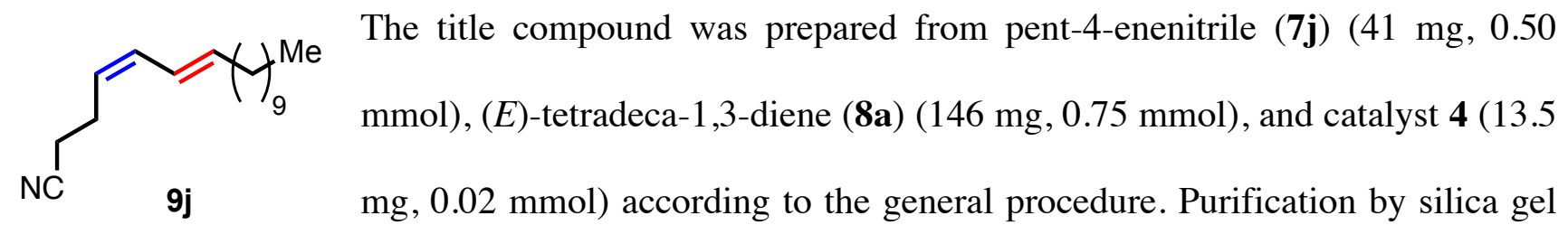
column chromatography using pure hexane (to elute unreacted $(E)$-tetradeca-1,3-diene $(\mathbf{8 a})$ ) followed by 20:1 hexane:Et ${ }_{2} \mathrm{O}$ provided the product as a colorless oil $\left(15 \mathrm{mg}, 12 \%\right.$ yield). ${ }^{1} \mathbf{H}$ NMR $(400 \mathrm{MHz}$, Chloroform- $d) \delta 6.28-6.19(\mathrm{~m}, 1 \mathrm{H}), 6.09(\mathrm{t}, J=10.9 \mathrm{~Hz}, 1 \mathrm{H}), 5.77(\mathrm{dt}, J=14.5,7.0 \mathrm{~Hz}, 1 \mathrm{H}), 5.27$ $(\mathrm{dt}, J=10.6,7.5 \mathrm{~Hz}, 1 \mathrm{H}), 2.53(\mathrm{q}, J=7.3 \mathrm{~Hz}, 2 \mathrm{H}), 2.43-2.37(\mathrm{~m}, 2 \mathrm{H}), 2.10(\mathrm{q}, J=7.1 \mathrm{~Hz}, 2 \mathrm{H})$, $1.43-1.35(\mathrm{~m}, 2 \mathrm{H}), 1.26(\mathrm{~s}, 14 \mathrm{H}), 0.90-0.86(\mathrm{~m}, 3 \mathrm{H}) ;{ }^{\mathbf{1 3}} \mathbf{C}$ NMR (101 MHz, Chloroform-d) $\delta$ 
$137.69,131.94,124.53,124.03,119.46,33.07,32.06,29.77,29.75,29.65,29.49,29.40,29.36,23.80$, 22.84, 17.71, 14.28. HRMS (FAB+) $\mathrm{m} / z$ Calcd for $\mathrm{C}_{17} \mathrm{H}_{30} \mathrm{~N}[\mathrm{M}+\mathrm{H}]^{+}$248.2378, found 248.2360.

\section{$(8 Z, 10 E)$-11,15-dimethylhexadeca-8,10,14-trien-1-ol (9k)}

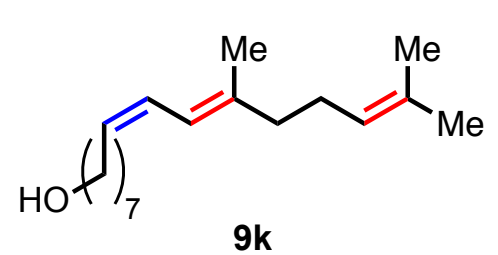

The title compound was prepared from non-8-en-1-ol (7a) $(28 \mathrm{mg}$, $0.20 \mathrm{mmol})$, (E)-4,8-dimethylnona-1,3,7-triene (8b) (45 $\mathrm{mg}, 0.3$ $\mathrm{mmol})$, and catalyst $4(2.7 \mathrm{mg}, 0.004 \mathrm{mmol})$ according to the general procedure. Purification by silica gel column chromatography using pure hexane ((E)-4,8dimethylnona-1,3,7-triene (8b)) followed by 20:1 hexane: $\mathrm{Et}_{2} \mathrm{O}$ provided the product as a colorless oil (53 mg, >95\% yield). ${ }^{1} \mathbf{H}$ NMR (500 MHz, Chloroform- $d$ ) $\delta 6.17(\mathrm{tt}, J=11.2,1.5 \mathrm{~Hz}, 1 \mathrm{H}), 6.06(\mathrm{dt}$, $J=11.4,1.1 \mathrm{~Hz}, 1 \mathrm{H}), 5.34(\mathrm{dt}, J=10.8,7.5 \mathrm{~Hz}, 1 \mathrm{H}), 5.16-5.06(\mathrm{~m}, 1 \mathrm{H}), 3.62(\mathrm{t}, J=6.7 \mathrm{~Hz}, 2 \mathrm{H})$, $2.18-2.14(\mathrm{~m}, 2 \mathrm{H}), 2.12-2.05(\mathrm{~m}, 4 \mathrm{H}), 1.74(\mathrm{~s}, 3 \mathrm{H}), 1.68(\mathrm{~s}, 3 \mathrm{H}), 1.61(\mathrm{~s}, 3 \mathrm{H}), 1.57-1.53(\mathrm{~m}, 2 \mathrm{H})$, $1.40-1.31(\mathrm{~m}, 8 \mathrm{H}) ;{ }^{13} \mathbf{C}$ NMR $(126 \mathrm{MHz}$, Chloroform- $d) \delta 138.59,131.72,130.08,124.79,124.23$, $120.04,63.14,40.40,32.89,29.79,29.44,29.37,27.63,26.81,25.82,17.84,16.57$; HRMS (FAB+) $m / z$ calcd for $\mathrm{C}_{18} \mathrm{H}_{31} \mathrm{O}\left[(\mathrm{M}+\mathrm{H})-\mathrm{H}_{2}\right]^{+} 263.2375$, found 263.2375.

\section{(5E,7Z)-15-hydroxypentadeca-5,7-dien-1-yl acetate (9l)}

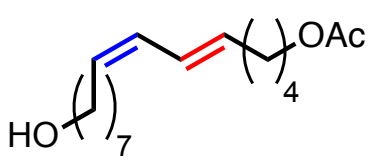

9l
The title compound was prepared from non-8-en-1-ol (7a) $(71 \mathrm{mg}, 0.50$ mmol), (E)-octa-5,7-dien-1-yl acetate (8c) (126 mg, $0.75 \mathrm{mmol})$, and catalyst $4(13.5 \mathrm{mg}, 0.02 \mathrm{mmol})$ according to the general procedure. Purification by

silica gel column chromatography using 5:1 hexane: $\mathrm{Et}_{2} \mathrm{O}$ provided the product as a colorless oil (105 $\mathrm{mg}, 75 \%$ yield). ${ }^{1} \mathbf{H}$ NMR (400 MHz, Chloroform- $d$ ) $\delta 6.29(\mathrm{ddt}, J=14.9,11.0,1.1 \mathrm{~Hz}, 1 \mathrm{H}), 5.92(\mathrm{t}$, $J=10.9 \mathrm{~Hz}, 1 \mathrm{H}), 5.61(\mathrm{dt}, J=14.5,7.0 \mathrm{~Hz}, 1 \mathrm{H}), 5.29(\mathrm{q}, J=7.8 \mathrm{~Hz}, 1 \mathrm{H}), 4.05(\mathrm{td}, J=6.6,1.3 \mathrm{~Hz}$, 
$2 \mathrm{H}), 3.61(\mathrm{td}, J=6.6,1.8 \mathrm{~Hz}, 2 \mathrm{H}), 2.16-2.10(\mathrm{~m}, 4 \mathrm{H}), 2.03(\mathrm{~d}, J=1.2 \mathrm{~Hz}, 3 \mathrm{H}), 1.67-1.41(\mathrm{~m}, 8 \mathrm{H})$, $1.33-1.28(\mathrm{~m}, 6 \mathrm{H}) ;{ }^{13} \mathbf{C}$ NMR (101 MHz, Chloroform- $d$ ) $\delta$ 171.41, 133.76, 130.51, 128.54, 126.26, $64.53,63.07,32.85,32.48,29.71,29.38,29.28,28.20,27.75,25.80,25.78,21.12 ;$ HRMS (FAB+) $m / z$ Calcd for $\mathrm{C}_{17} \mathrm{H}_{31} \mathrm{O}_{3}[\mathrm{M}+\mathrm{H}]^{+}$283.2273, found 283.2273.

\section{$(8 Z, 10 E)$-11-phenylundeca-8,10-dien-1-ol (9m)}

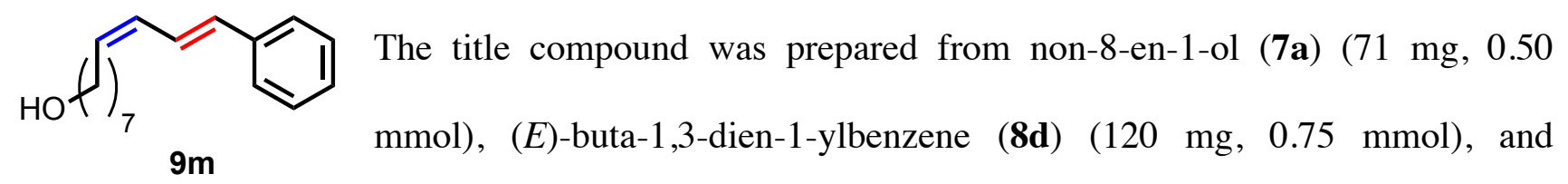

catalyst $4(13.5 \mathrm{mg}, 0.02 \mathrm{mmol})$ according to the general procedure. The crude mixture was purified by semi-preparative scale HPLC $(9.4 \mathrm{~mm} \times 250 \mathrm{~mm}, 5 \mu \mathrm{m}$ Agilent Eclipse XDB-C18 column, detection at $230 \mathrm{~nm}$, flow rate $3 \mathrm{~mL} / \mathrm{min}, \mathrm{H} 2 \mathrm{O} / \mathrm{MeCN}$, gradient: ramp 90-100\% $\mathrm{MeCN}$ over $4 \mathrm{~min}$, $100 \% \mathrm{MeCN}$ for $8 \mathrm{~min}, \mathrm{tR}(\mathrm{min})=8.4 \mathrm{~min}$ to afford a colorless liquid $\left(19 \mathrm{mg}, 15 \%\right.$ yield). ${ }^{1} \mathbf{H}$ NMR $(400 \mathrm{MHz}$, Chloroform- $d) \delta 7.41(\mathrm{dd}, J=8.4,1.1 \mathrm{~Hz}, 2 \mathrm{H}), 7.31(\mathrm{td}, J=7.0,6.6,1.6 \mathrm{~Hz}, 2 \mathrm{H}), 7.24-$ 7.19 (m, 1H), 7.06 (ddd, $J=15.6,11.1,1.1 \mathrm{~Hz}, 1 \mathrm{H}), 6.52(\mathrm{~d}, J=15.6 \mathrm{~Hz}, 1 \mathrm{H}), 6.20-6.11(\mathrm{~m}, 1 \mathrm{H})$, $5.53(\mathrm{dtt}, J=10.9,7.7,1.0 \mathrm{~Hz}, 1 \mathrm{H}), 3.64(\mathrm{q}, J=6.5 \mathrm{~Hz}, 2 \mathrm{H}), 2.33-2.24(\mathrm{~m}, 2 \mathrm{H}), 1.58(\mathrm{~d}, J=6.8 \mathrm{~Hz}$, 2H), $1.48-1.41(\mathrm{~m}, 2 \mathrm{H}), 1.42-1.33(\mathrm{~m}, 6 \mathrm{H}) ;{ }^{13} \mathrm{C}$ NMR (101 MHz, Chloroform- $d$ ) $\delta$ 137.79, $133.42,132.11,128.86,128.72,127.48,126.44,124.60,63.22,32.92,29.76,29.44,29.35,28.12$, 25.84; HRMS (FAB+) $m / z$ Calcd for $\mathrm{C}_{17} \mathrm{H}_{24} \mathrm{O}[\mathrm{M}]^{+} 244.1827$, found 244.1835 . 
(8Z,10E)-11-(4-methoxyphenyl)undeca-8,10-dien-1-ol (9n)

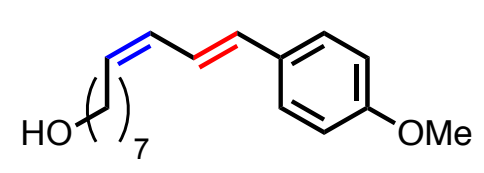

$9 n$

The title compound was prepared from non-8-en-1-ol (7a) (71 mg, 0.50 mmol), (E)-1-(buta-1,3-dien-1-yl)-4-methoxybenzene (8e) (120 mg, $0.75 \mathrm{mmol})$, and catalyst $4(13.5 \mathrm{mg}, 0.02 \mathrm{mmol})$ according to the general procedure. Purification by silica gel column chromatography using 5:1 hexane: $\mathrm{Et}_{2} \mathrm{O}$ provided the product as a white solid (34 mg, 25\% yield). ${ }^{1} \mathbf{H}$ NMR $(400 \mathrm{MHz}$, Chloroform- $d) \delta 7.35(\mathrm{~d}, J=$ $8.7 \mathrm{~Hz}, 2 \mathrm{H}), 6.96-6.88(\mathrm{~m}, 1 \mathrm{H}), 6.88-6.82(\mathrm{~m}, 2 \mathrm{H}), 6.47(\mathrm{~d}, J=15.5 \mathrm{~Hz}, 1 \mathrm{H}), 6.13(\mathrm{t}, J=10.9 \mathrm{~Hz}$, 1H), $5.46(\mathrm{dt}, J=10.6,7.7 \mathrm{~Hz}, 1 \mathrm{H}), 3.81(\mathrm{~s}, 3 \mathrm{H}), 3.64(\mathrm{t}, J=6.6 \mathrm{~Hz}, 2 \mathrm{H}), 2.27(\mathrm{q}, J=6.7 \mathrm{~Hz}, 2 \mathrm{H})$, $1.60-1.54(\mathrm{~m}, 2 \mathrm{H}), 1.45-1.40(\mathrm{~m}, 2 \mathrm{H}), 1.35(\mathrm{~s}, 6 \mathrm{H}) ;{ }^{13} \mathbf{C}$ NMR (101 MHz, Chloroform-d) $\delta$ $159.19,132.25,131.64,130.61,129.00,127.62,122.68,114.17,63.21,55.45,32.91,29.80,29.44$, 29.35, 28.08, 25.83; HRMS (FAB+) $m / z$ Calcd for $\mathrm{C}_{18} \mathrm{H}_{26} \mathrm{O}_{2}[\mathrm{M}]^{+}$274.1933, found 274.1948.

\section{(5E,7Z)-dodeca-5,7-dien-1-yl acetate (9o)}

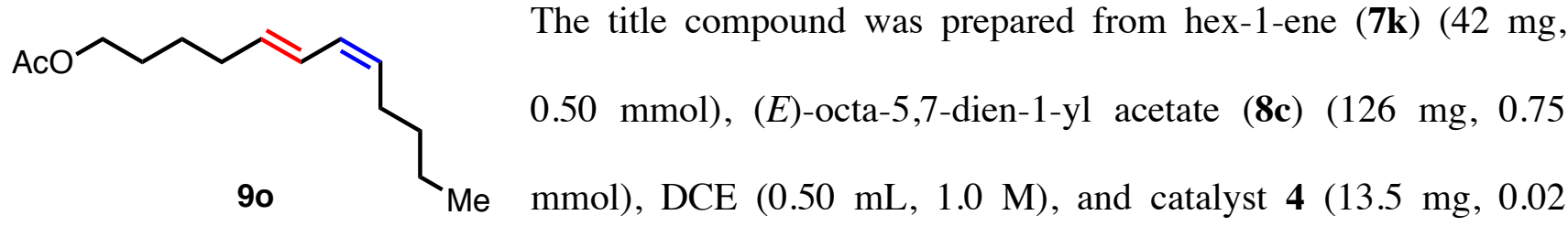

mmol) according to the general procedure. The crude mixture was purified by preparative scale HPLC $(21.2 \mathrm{~mm} \times 250 \mathrm{~mm}, 5 \mu \mathrm{m}$ ACE C18 column, detection at $230 \mathrm{~nm}$, flow rate $30 \mathrm{~mL} / \mathrm{min}$, H2O/MeCN, gradient: ramp 65-100\% MeCN over $12 \mathrm{~min}, 100 \% \mathrm{MeCN}$ for $13 \mathrm{~min}$, tR $(\min )=17.5$ min) to afford a colorless liquid (36 mg, 30\% yield). ${ }^{1} \mathbf{H}$ NMR (400 MHz, Chloroform- $d$ ) $\delta 6.37-$ $6.26(\mathrm{~m}, 1 \mathrm{H}), 5.94(\mathrm{t}, J=10.9 \mathrm{~Hz}, 1 \mathrm{H}), 5.62(\mathrm{dt}, J=14.6,7.0 \mathrm{~Hz}, 1 \mathrm{H}), 5.32(\mathrm{dt}, J=10.7,7.6 \mathrm{~Hz}$, $1 \mathrm{H}), 4.06(\mathrm{t}, J=6.7 \mathrm{~Hz}, 2 \mathrm{H}), 2.19-2.10(\mathrm{~m}, 4 \mathrm{H}), 2.04(\mathrm{~s}, 3 \mathrm{H}), 1.68-1.61(\mathrm{~m}, 2 \mathrm{H}), 1.46(\mathrm{p}, J=7.6$ Hz, 2H), $1.40-1.29(\mathrm{~m}, 4 \mathrm{H}), 0.90(\mathrm{t}, J=7.1 \mathrm{~Hz}, 3 \mathrm{H}) ;{ }^{13} \mathrm{C}$ NMR (101 MHz, Chloroform-d) $\delta$ 171.37, 
$133.73,130.67,128.50,126.32,64.56,32.53,32.02,28.25,27.55,25.84,22.47,21.16,14.12$; HRMS $(\mathrm{FAB}+) \mathrm{m} / \mathrm{z}$ Calcd for $\mathrm{C}_{14} \mathrm{H}_{24} \mathrm{O}_{2}[\mathrm{M}]^{+} 224.1776$, found 224.1783 .

\section{$(9 Z, 11 E)$-tetradeca-9,11-dien-1-yl acetate $(9 p)$}

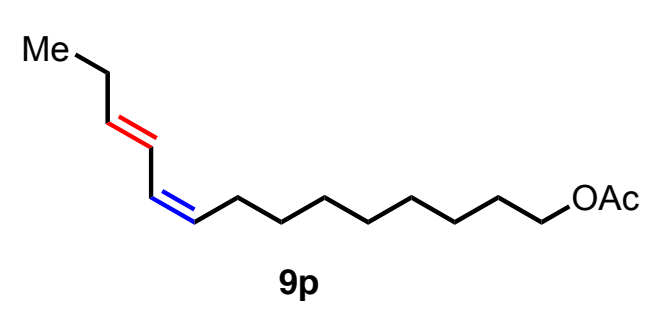

The title compound was prepared from dec-9-en-1-yl acetate (7l) $(99 \mathrm{mg}, 0.50 \mathrm{mmol}),(E)$-hexa-1,3-diene $(\mathbf{8 g})(62 \mathrm{mg}$, $0.75 \mathrm{mmol})$, DCE $(0.50 \mathrm{~mL}, 1.0 \mathrm{M})$, and catalyst $4(13.5 \mathrm{mg}$, $0.02 \mathrm{mmol}$ ) according to the general procedure. The crude mixture was purified by preparative scale HPLC $(21.2 \mathrm{~mm}$ x $250 \mathrm{~mm}, 5 \mu \mathrm{m}$ ACE C18 column, detection at $230 \mathrm{~nm}$, flow rate $50 \mathrm{~mL} / \mathrm{min}, \mathrm{H} 2 \mathrm{O} / \mathrm{MeCN}$, gradient: ramp 70-100\% MeCN over $6 \mathrm{~min}$, $100 \% \mathrm{MeCN}$ for $9 \mathrm{~min}$, tR $(\mathrm{min})=10.8 \mathrm{~min})$ to afford a colorless liquid $(70 \mathrm{mg}, 56 \%$ yield $) .{ }^{1} \mathbf{H}$ NMR (400 MHz, Chloroform- $d$ ) $\delta 6.29(\mathrm{ddq}, J=15.1,11.0,1.5 \mathrm{~Hz}, 1 \mathrm{H}), 5.94(\mathrm{t}, J=10.9 \mathrm{~Hz}, 1 \mathrm{H})$, $5.69(\mathrm{dt}, J=15.0,6.6 \mathrm{~Hz}, 1 \mathrm{H}), 5.29(\mathrm{dt}, J=10.7,7.6 \mathrm{~Hz}, 1 \mathrm{H}), 4.04(\mathrm{t}, J=6.8 \mathrm{~Hz}, 2 \mathrm{H}), 2.13(\mathrm{dq}, J=$ 15.0, 7.1 Hz, 4H), $2.04(\mathrm{~s}, 3 \mathrm{H}), 1.63-1.59(\mathrm{~m}, 2 \mathrm{H}), 1.39-1.27(\mathrm{~m}, 10 \mathrm{H}), 1.01(\mathrm{t}, J=7.5 \mathrm{~Hz}, 3 \mathrm{H})$; ${ }^{13}$ C NMR (101 MHz, Chloroform- $d$ ) $\delta$ 171.40, 136.30, 130.21, 128.74, 124.79, 64.79, 29.82, 29.51, $29.35,29.29,28.73,27.79,26.03,21.18,13.81$; HRMS (FAB+) $\mathrm{m} / \mathrm{z}$ Calcd for $\mathrm{C}_{16} \mathrm{H}_{28} \mathrm{O}_{2}[\mathrm{M}]^{+}$ 252.2089, found 252.2101.

\section{$(11 E, 13 Z, 15 E)$-hexacosa-11,13,15-triene (28a)}

Me ()$_{9}$ 28a $4_{9}$ me $\left.\mathrm{mg}, 0.75 \mathrm{mmol}\right), \mathrm{DCE}(0.50 \mathrm{~mL}, 1.0 \mathrm{M})$ and catalyst 4 (13.5 mg, 0.02 mmol) according to the general procedure. Purification by silica gel column chromatography using pure hexane provided a mixture of the starting $(E)$-tetradeca-1,3-diene $(\mathbf{8 a})$ and the desired product as a colorless liquid (62\% yield by ${ }^{1} \mathrm{H}$ NMR). The product identity was assigned based on analogy to the 
${ }^{1} \mathrm{H}$ NMR spectra of $\mathbf{2 8 b}$ and $\mathbf{2 8 c}$, and no attempt was made to purify this mixture further. ${ }^{\mathbf{1}} \mathbf{H}$ NMR $(500 \mathrm{MHz}$, Chloroform- $d) \delta 6.53-6.42(\mathrm{~m}, 2 \mathrm{H}), 5.88-5.81(\mathrm{~m}, 2 \mathrm{H}), 5.69(\mathrm{dt}, J=14.7,7.1 \mathrm{~Hz}, 2 \mathrm{H})$, $2.12(\mathrm{q}, J=6.9 \mathrm{~Hz}, 4 \mathrm{H}), 1.42-1.37(\mathrm{~m}, 4 \mathrm{H}), 1.27(\mathrm{~s}, 28 \mathrm{H}), 0.89(\mathrm{t}, J=6.9 \mathrm{~Hz}, 6 \mathrm{H})$.

\section{$(5 E, 7 Z, 9 E)$-tetradeca-5,7,9-triene-1,14-diyl diacetate (28b)}

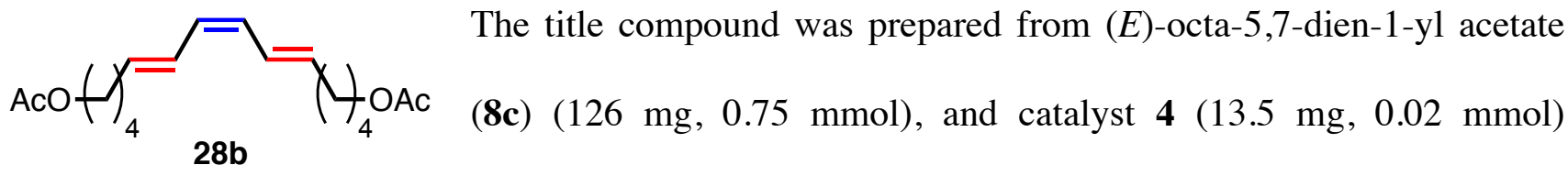

according to the general procedure. Purification by silica gel column chromatography using 1:1 hexane: $\mathrm{Et}_{2} \mathrm{O}$ provided the product as a colorless oil $\left(85 \mathrm{mg}, 74 \%\right.$ yield). ${ }^{1} \mathbf{H} \mathbf{~ N M R}(400 \mathrm{MHz}$, Chloroform- $d) \delta 6.55-6.41(\mathrm{~m}, 2 \mathrm{H}), 5.89-5.79(\mathrm{~m}, 2 \mathrm{H}), 5.66(\mathrm{dt}, J=14.6,7.1 \mathrm{~Hz}, 2 \mathrm{H}), 4.05(\mathrm{t}, J=$ $6.7 \mathrm{~Hz}, 4 \mathrm{H}), 2.15(\mathrm{qd}, J=7.4,1.1 \mathrm{~Hz}, 4 \mathrm{H}), 2.03(\mathrm{~s}, 6 \mathrm{H}), 1.65-1.59(\mathrm{~m}, 4 \mathrm{H}), 1.46(\mathrm{dd}, J=8.9,6.2$ $\mathrm{Hz}, 4 \mathrm{H}) ;{ }^{13} \mathrm{C}$ NMR (101 MHz, Chloroform-d) $\delta 171.34,134.80,127.84,126.34,64.48,32.57,28.22$, 25.75, 21.13; HRMS (FAB+) m/z calcd for $\mathrm{C}_{18} \mathrm{H}_{27} \mathrm{O}_{4}\left[(\mathrm{M}+\mathrm{H})-\mathrm{H}_{2}\right]^{+} 307.1909$, found 307.1900.

\section{(5E,7Z,9E)-tetradeca-5,7,9-triene-1,14-diol (28c)}

The title compound was prepared from (E)-octa-5,7-dien-1-ol (8f) (95 $\mathrm{HO}\left(\mathrm{T}_{4}{ }_{28 \mathrm{c}}\left(\mathrm{f}_{4} \mathrm{OH} \mathrm{mg}, 0.75 \mathrm{mmol}\right)\right.$, and catalyst $4(13.5 \mathrm{mg}, 0.02 \mathrm{mmol})$ according to the general procedure. Purification by silica gel column chromatography using 1:4 hexane: $\mathrm{Et}_{2} \mathrm{O}$ provided the product as a colorless oil (48 mg, 57\% yield). ${ }^{1} \mathbf{H}$ NMR (400 MHz, Chloroform- $d$ ) $\delta 6.57-6.42$ $(\mathrm{m}, 2 \mathrm{H}), 5.91-5.80(\mathrm{~m}, 2 \mathrm{H}), 5.69(\mathrm{dt}, J=14.6,7.0 \mathrm{~Hz}, 2 \mathrm{H}), 3.66(\mathrm{q}, J=6.2 \mathrm{~Hz}, 4 \mathrm{H}), 2.20-2.13(\mathrm{~m}$, 4H), $1.64-1.60(\mathrm{~m}, 2 \mathrm{H}), 1.59-1.53(\mathrm{~m}, 2 \mathrm{H}), 1.53-1.45(\mathrm{~m}, 4 \mathrm{H}), 1.26(\mathrm{t}, J=5.2 \mathrm{~Hz}, 2 \mathrm{H}) ;{ }^{13} \mathrm{C}$ NMR (101 MHz, Chloroform-d) $\delta$ 135.07, 127.84, 126.25, 63.00, 32.75, 32.41, 25.57; HRMS $(\mathrm{FAB}+) \mathrm{m} / z$ calcd for $\mathrm{C}_{14} \mathrm{H}_{24} \mathrm{O}_{2}[\mathrm{M}]^{+} 224.1776$, found 224.1766 . 
$(((((1 S, 2 R, 3 S, 5 R)-3-((1 Z, 3 E)$-tetradeca-1,3-dien-1-yl)-5-vinylcyclopentane-1,2diyl)bis(methylene))bis(oxy))bis(methylene))dibenzene (30a)

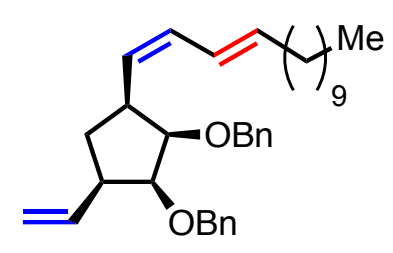

$( \pm)-30 a$

The title compound was prepared from $(5 R, 6 S)-5,6$-bis((benzyloxy)methyl)-bicyclo[2.2.1]hept-2-ene (29a) (33 mg, $0.10 \mathrm{mmol})$, (E)-tetradeca-1,3-diene (8a) (29 mg, $0.15 \mathrm{mmol})$, and catalyst 4 (1.4 mg, $0.002 \mathrm{mmol}$ ) according to the general procedure. Purification by silica gel column chromatography using pure hexane (to elute unreacted $(E)$-tetradeca-1,3-diene $(\mathbf{8 a})$ ) followed by 20:1 hexane: $\mathrm{Et}_{2} \mathrm{O}$ provided the product as a colorless oil $(53 \mathrm{mg},>95 \%$ yield $) .{ }^{1} \mathbf{H}$ NMR $(500$ $\mathrm{MHz}$, Chloroform- $d) \delta 7.40-7.25(\mathrm{~m}, 10 \mathrm{H}), 6.37-6.28(\mathrm{~m}, 1 \mathrm{H}), 5.99-5.85(\mathrm{~m}, 2 \mathrm{H}), 5.68(\mathrm{dt}, J=$ 14.5, 7.0 Hz, 1H), $5.36-5.26(\mathrm{~m}, 1 \mathrm{H}), 5.00(\mathrm{ddd}, J=17.1,2.0,1.0 \mathrm{~Hz}, 1 \mathrm{H}), 4.93(\mathrm{dd}, J=10.1,2.1$ $\mathrm{Hz}, 1 \mathrm{H}), 4.43-4.33(\mathrm{~m}, 4 \mathrm{H}), 3.54-3.44(\mathrm{~m}, 4 \mathrm{H}), 3.23(\mathrm{p}, J=9.0 \mathrm{~Hz}, 1 \mathrm{H}), 2.77$ (p, $J=8.5 \mathrm{~Hz}, 1 \mathrm{H})$, $2.48(\mathrm{dp}, J=13.3,6.9 \mathrm{~Hz}, 2 \mathrm{H}), 2.09(\mathrm{q}, J=7.0 \mathrm{~Hz}, 2 \mathrm{H}), 2.03(\mathrm{dt}, J=12.9,8.2 \mathrm{~Hz}, 1 \mathrm{H}), 1.62-1.55$ $(\mathrm{m}, 1 \mathrm{H}), 1.42-1.35(\mathrm{~m}, 2 \mathrm{H}), 1.28(\mathrm{~s}, 14 \mathrm{H}), 0.90(\mathrm{t}, J=7.0 \mathrm{~Hz}, 3 \mathrm{H}) ;{ }^{13} \mathbf{C} \mathbf{N M R}(126 \mathrm{MHz}$, Chloroform- $d) \delta 140.88,138.74,138.71,135.34,132.10,128.94,128.40,128.36,127.97,127.58$, $127.54,125.84,114.27,73.34,69.01,68.88,45.97,45.86,45.73,39.12,38.93,33.08,32.07,29.79$, 29.78, 29.69, 29.59, 29.50, 29.46, 22.85, 14.29; HRMS (FAB+) $m / z$ Calcd for $\mathrm{C}_{30} \mathrm{H}_{45} \mathrm{O}_{2}[\mathrm{M}-\mathrm{Bn}]^{+}$ 437.3420, found 437.3412 .

$((((3 R, 4 S, 5 Z, 7 E)$-octadeca-1,5,7-triene-3,4-diyl)bis(oxy))bis(methylene))dibenzene (30b)<smiles>C=C[C@@H](OCc1ccccc1)[C@H](/C=C\C=C\C(C)C)OCc1ccccc1</smiles>

$( \pm)-30 b$

The title compound was prepared from $(3 R, 4 S)-3,4-$ bis(benzyloxy)cyclobut-1-ene (29b) (27 mg, $0.10 \mathrm{mmol}),(E)$-tetradeca1,3-diene (8a) (29 mg, $0.15 \mathrm{mmol})$, and catalyst 4 (1.4 mg, $0.002 \mathrm{mmol})$ 
according to the general procedure. Purification by silica gel column chromatography using pure hexane (to elute unreacted (E)-tetradeca-1,3-diene (8a)) followed by 20:1 hexane:Et ${ }_{2} \mathrm{O}$ provided the product as a colorless oil (41 mg, 89\% yield). ${ }^{1} \mathbf{H}$ NMR $(500 \mathrm{MHz}$, Chloroform- $d$ ) $\delta 7.32$ (dt, $J=$ 10.6, $7.6 \mathrm{~Hz}, 8 \mathrm{H}), 7.29-7.24(\mathrm{~m}, 2 \mathrm{H}), 6.25(\mathrm{t}, J=11.0 \mathrm{~Hz}, 1 \mathrm{H}), 6.17-6.09(\mathrm{~m}, 1 \mathrm{H}), 5.88$ (ddd, $J=$ 17.3, 10.4, 7.7 Hz, 1H), $5.75(\mathrm{dt}, J=14.5,7.0 \mathrm{~Hz}, 1 \mathrm{H}), 5.35-5.25(\mathrm{~m}, 3 \mathrm{H}), 4.65(\mathrm{dd}, J=12.3,2.7$ $\mathrm{Hz}, 2 \mathrm{H}), 4.49-4.41(\mathrm{~m}, 2 \mathrm{H}), 4.36(\mathrm{dd}, J=9.4,4.5 \mathrm{~Hz}, 1 \mathrm{H}), 3.87$ (dd, $J=7.7,4.5 \mathrm{~Hz}, 1 \mathrm{H}), 2.06(\mathrm{q}, J$ $=7.0 \mathrm{~Hz}, 2 \mathrm{H}), 1.40-1.33(\mathrm{~m}, 2 \mathrm{H}), 1.28(\mathrm{~s}, 14 \mathrm{H}), 0.89(\mathrm{t}, J=7.0 \mathrm{~Hz}, 3 \mathrm{H}) ;{ }^{13} \mathbf{C}$ NMR $(126 \mathrm{MHz}$, Chloroform-d) $\delta 138.82,138.81,137.80,135.60,133.60,128.32,128.31,127.94,127.71,127.47$, $127.43,125.97,125.56,118.97,82.77,76.31,70.54,70.12,32.97,32.07,29.80,29.79,29.69,29.51$, 29.43, 29.37, 22.85, 14.28; HRMS (FAB+) $\mathrm{m} / \mathrm{z}$ Calcd for $\mathrm{C}_{32} \mathrm{H}_{45} \mathrm{O}_{2}[\mathrm{M}+\mathrm{H}]^{+} 461.3420$, found 461.3423. 


\section{Computational Details}

DFT calculations were performed with Gaussian 09. ${ }^{5}$ Computed structures are illustrated using CYLView. ${ }^{6}$

Geometries were optimized with the $\mathrm{B}^{2} \mathrm{LYP}^{7}$ functional in the gas phase, using a mixed basis set of LANL2DZ (with ECP) for Ru and 6-31G(d) for all other atoms. Thermal corrections were calculated from unscaled vibrational frequencies at the same level of theory using a standard state of $298.15 \mathrm{~K}$ and $1 \mathrm{~mol} / \mathrm{L}$.

Gibbs free energies in Gaussian were calculated at the default pressure of $1 \mathrm{~atm}$ and corrected to the standard state in solution, $1 \mathrm{~mol} / \mathrm{L}$. The correction was made by adding $R T \ln \left(c_{\text {soln }} / c_{\text {gas }}\right)$, or about 1.84 $\mathrm{kcal} / \mathrm{mol}$, to the free energy of all structures, where $c_{\text {soln }}$ is the standard molar concentration in solution $(1 \mathrm{~mol} / \mathrm{L})$ and $c_{\text {gas }}$ is the standard molar concentration in the gas phase $(0.0446 \mathrm{~mol} / \mathrm{L})$.

Electronic energies were obtained from single point energy calculations performed with the M06 ${ }^{8}$ functional and a mixed basis set of SDD for Ru and $6-311+\mathrm{G}(\mathrm{d}, \mathrm{p})$ for all other atoms. The $\mathrm{SMD}^{9}$ solvation model for n-octanol was used in M06 single point energy calculations. This solvent was chosen to model reactions run neat with alkenes containing a polar functional group.

IRC calculations were performed to link each transition state to the relevant intermediates. These calculations confirmed that the nitrate ligand is bidentate in alkylidene ruthenium complexes, but shifts to monodentate coordination upon metallacyclobutane formation. We also found stable olefin $\pi$-complexes in some cases; these complexes are shown below in the SI (highlighted in red), but omitted from main text. 


\section{Vinylcarbene Conformations}

Figure S3: Conformations of vinylcarbene 14 (Pathway A)

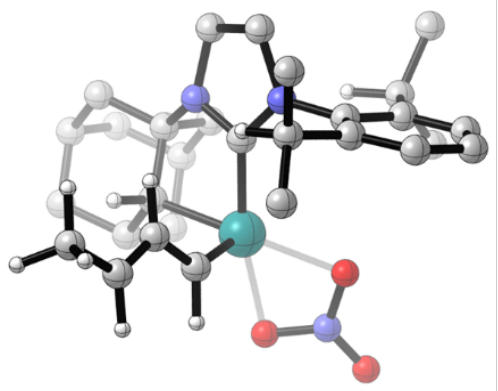

14

$G_{\text {rel }}=0.0$

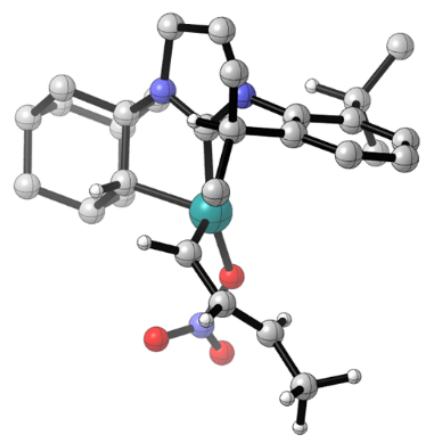

14d

$G_{\text {rel }}=4.7$

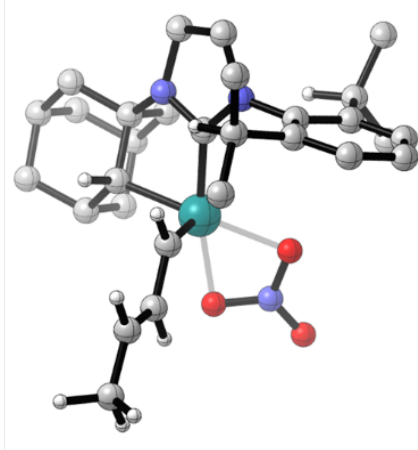

14b

$G_{\text {rel }}=0.4$

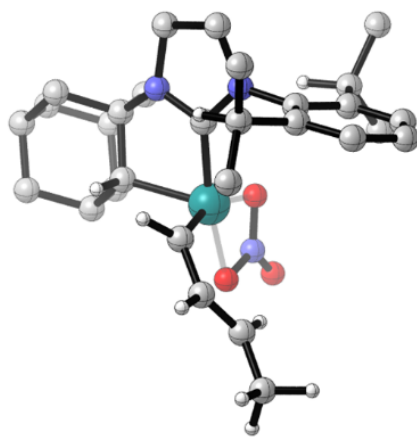

$14 \mathrm{e}$

$G_{\text {rel }}=4.8$

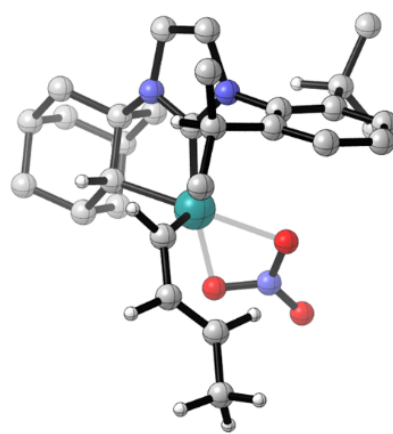

14c

$G_{\text {rel }}=3.9$

Conformational searches were performed and we were unable to locate an $\eta^{3}$-vinylcarbene complex as a local minimum. To estimate the relative energy of such a complex the $\mathrm{Ru}-\mathrm{C} 3$ distance was constrained to $2.56 \AA$ (the distance computed for $\eta^{3}$-vinylcarbene complex 31, see below), and all other coordinates were optimized. The resulting complex, 14-contrained, is $13.3 \mathrm{kcal} / \mathrm{mol}$ higher in energy than 14. 
Figure S4: S-cis conformations of non-chelated (SIMes) vinylcarbenes 31-34

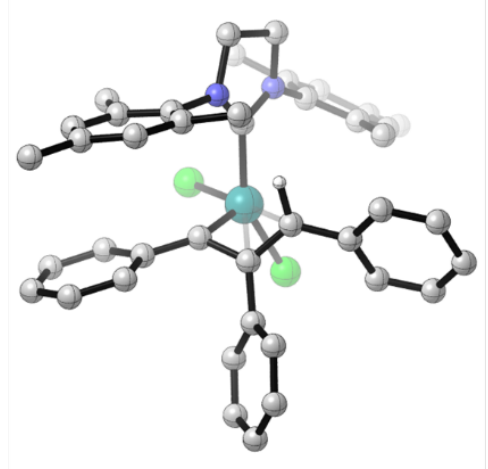

31

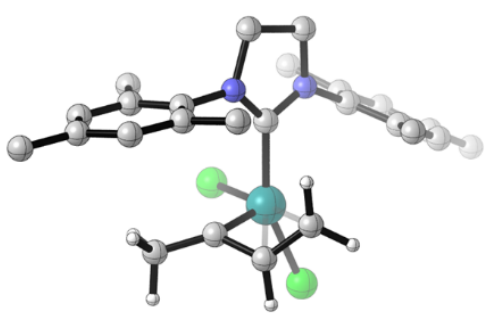

33

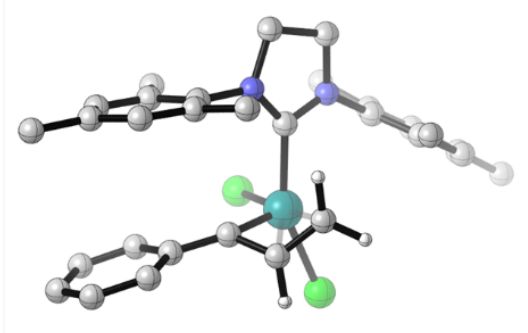

32

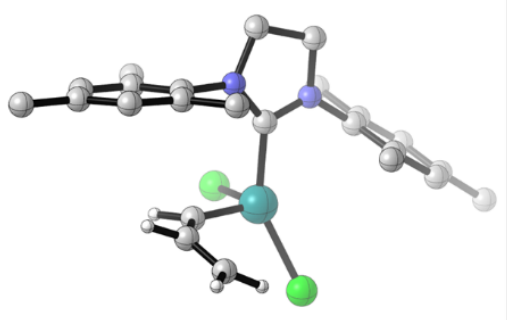

34

Vinylcarbene $\mathbf{3 1}$ is known to exist as an $\eta^{3}$-vinylcarbene complex based on an $\mathrm{x}$-ray crystal structure. Likewise, our calculations confirm the $\eta^{3}$-vinylcarbene structure to be the lowest-energy conformation for 31. Additionally, our calculations indicate that $\eta^{3}$-vinylcarbene complexes are favored for 1-phenyl-1-vinylcarbene 32 and 1-methyl-1-vinylcarbene 33. However, we were unable to locate an $\eta^{3}$ coordination mode for the parent vinylcarbene complex 34. Substitution at the 1position appears to be required for an $\eta^{3}$ coordination mode. 
Figure S5: Lowest-energy conformations of chelated 1-methyl-1-vinylcarbene 35

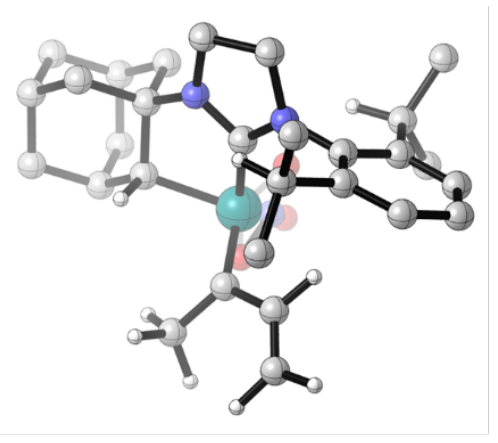

$35 \mathrm{a}$
$G_{\mathrm{rel}}=0.0$

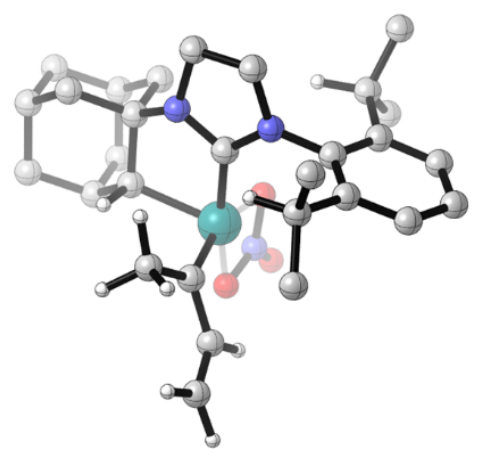

$35 \mathbf{b}$
$G_{\mathrm{rel}}=0.2$

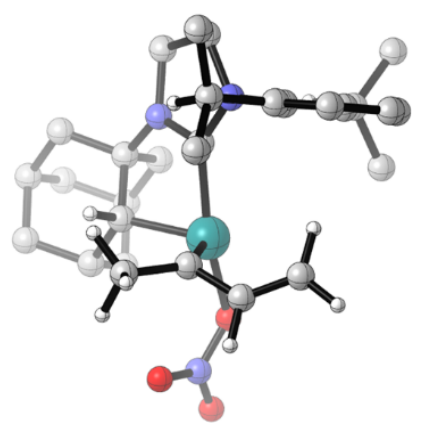

$35 \mathrm{c}$

$G_{\text {rel }}=0.3$

We also examined the conformations of 35, the 1-methyl analogue of vinylcarbene complex 14. Here also, we were unable to locate an $\eta^{3}$-vinylcarbene complex as a local minimum. Since an $\eta^{3}$ coordination mode would place the alkene ligand trans to the adamantly ligand, we speculate that $\eta^{3}$ coordination of chelated catalysts may be less favorable due to the strong trans effect of the chelating alkyl ligand.

\section{Additional Intermediates on Diene-First Pathway A}

Scheme S3: Complete Pathway A including olefin m-complexes

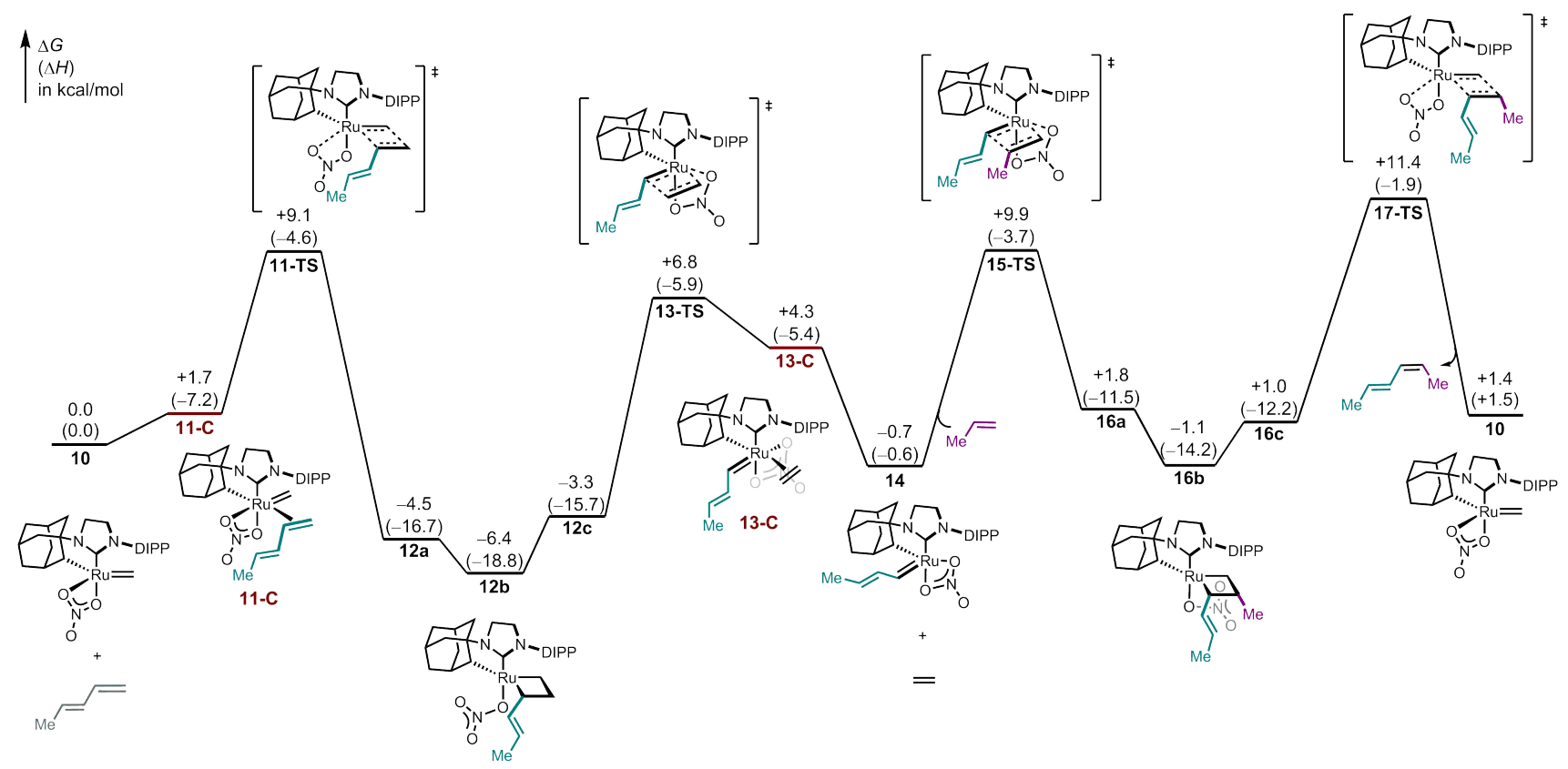


Figure S6: Conformations of 2-vinylmetallacyclobutane 12

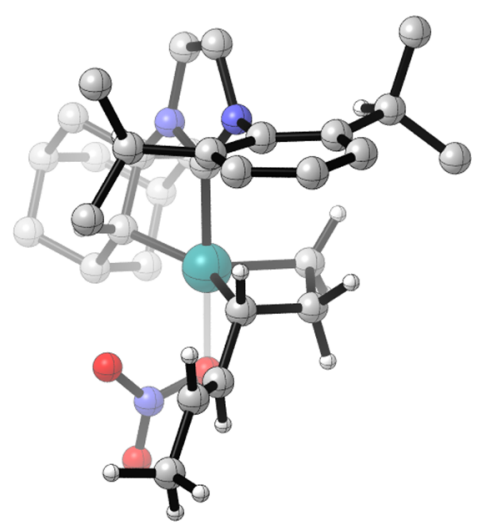

$12 a$

$\Delta G=-4.5$

(from methylidene 10)

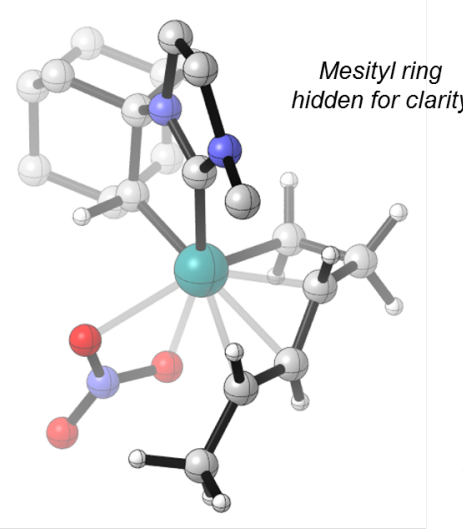

$12 d$

$\Delta G=-5.2$

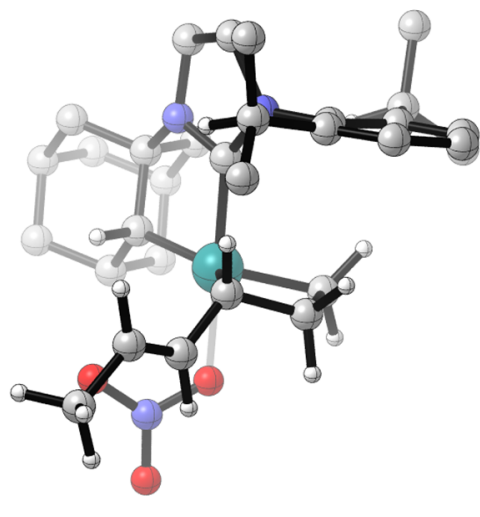

$12 \mathrm{~b}$

$\Delta G=-6.4$

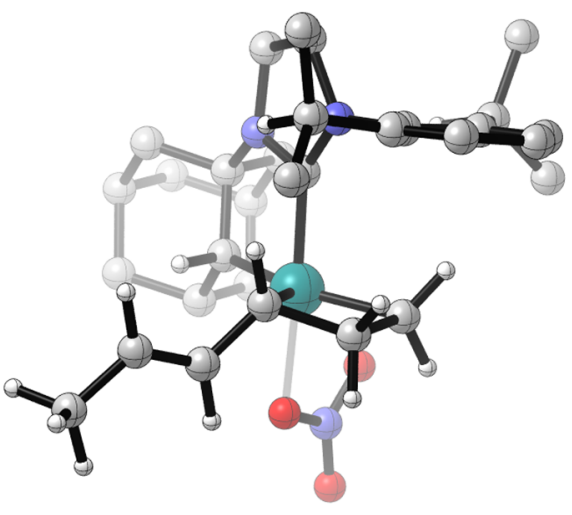

12c

$\Delta G=-3.3$

(leads to vinylcarbene 14)

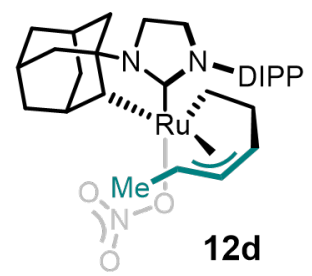

$\eta^{3}$-vinyImetallacyclobutane 
Figure S7: Conformations of 2-vinylmetallacyclobutane 16

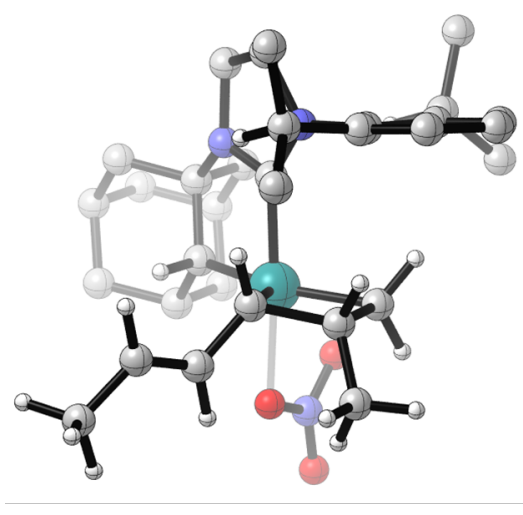

$16 a$

$\Delta G=+1.8$

(from vinylcarbene 14)

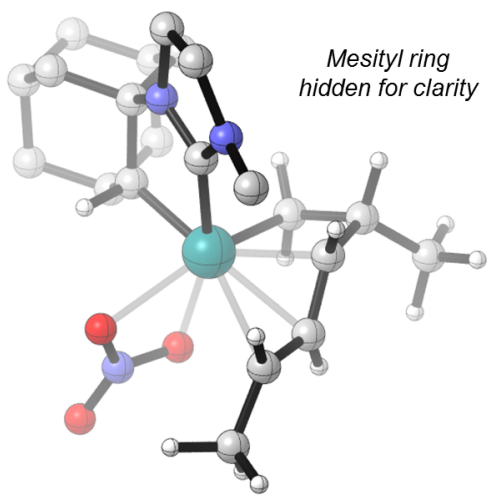

16d

$\Delta G=-2.8$

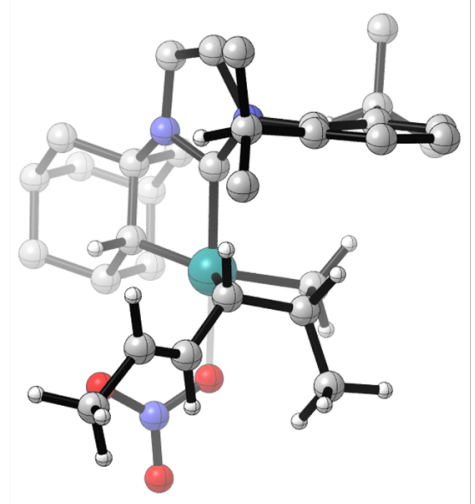

$16 \mathrm{~b}$

$\Delta G=-1.1$

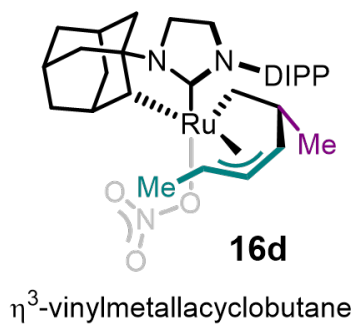

$\eta^{3}$-vinylmetallacyclobutane

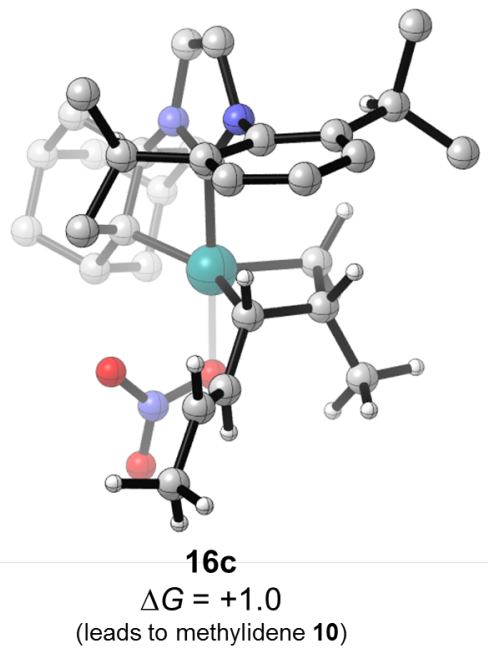

Although we were able to locate $\eta^{3}$-vinylmetallacyclobutane intermediates $\mathbf{1 2 d}$ and $\mathbf{1 6 d}$, they are similar in energy to other conformations of the metallacycles $\mathbf{1 2}$ and 16, and we do not believe they impact the overall reactivity. 


\section{Additional Intermediates on Alkene-First Pathway B}

Scheme S4: Complete Pathway B including olefin m-complexes

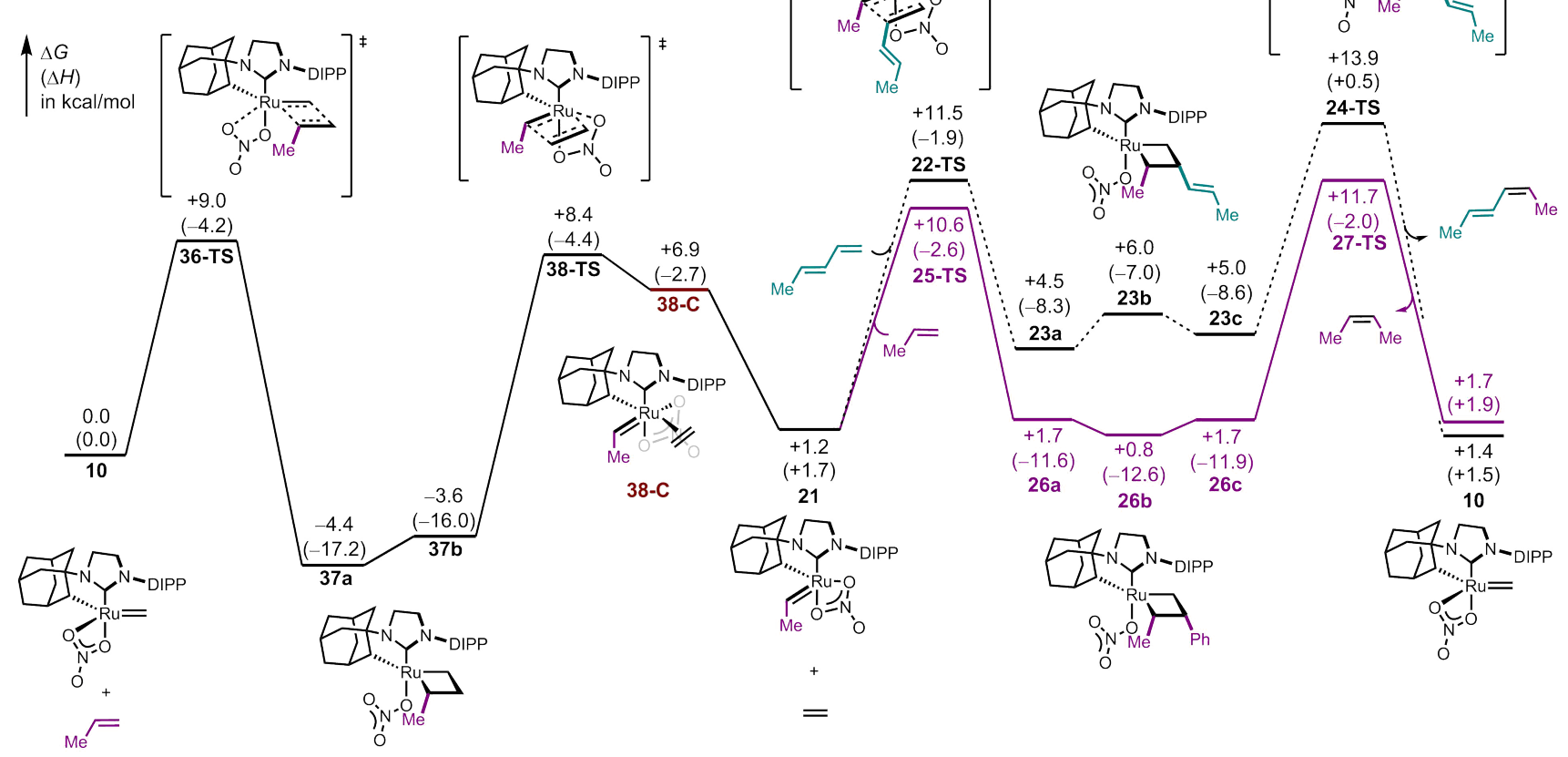




\section{Reaction Pathway C Starting from Diastereomeric Methylidene Complex 39}

Scheme S5: Diastereomeric Pathway C

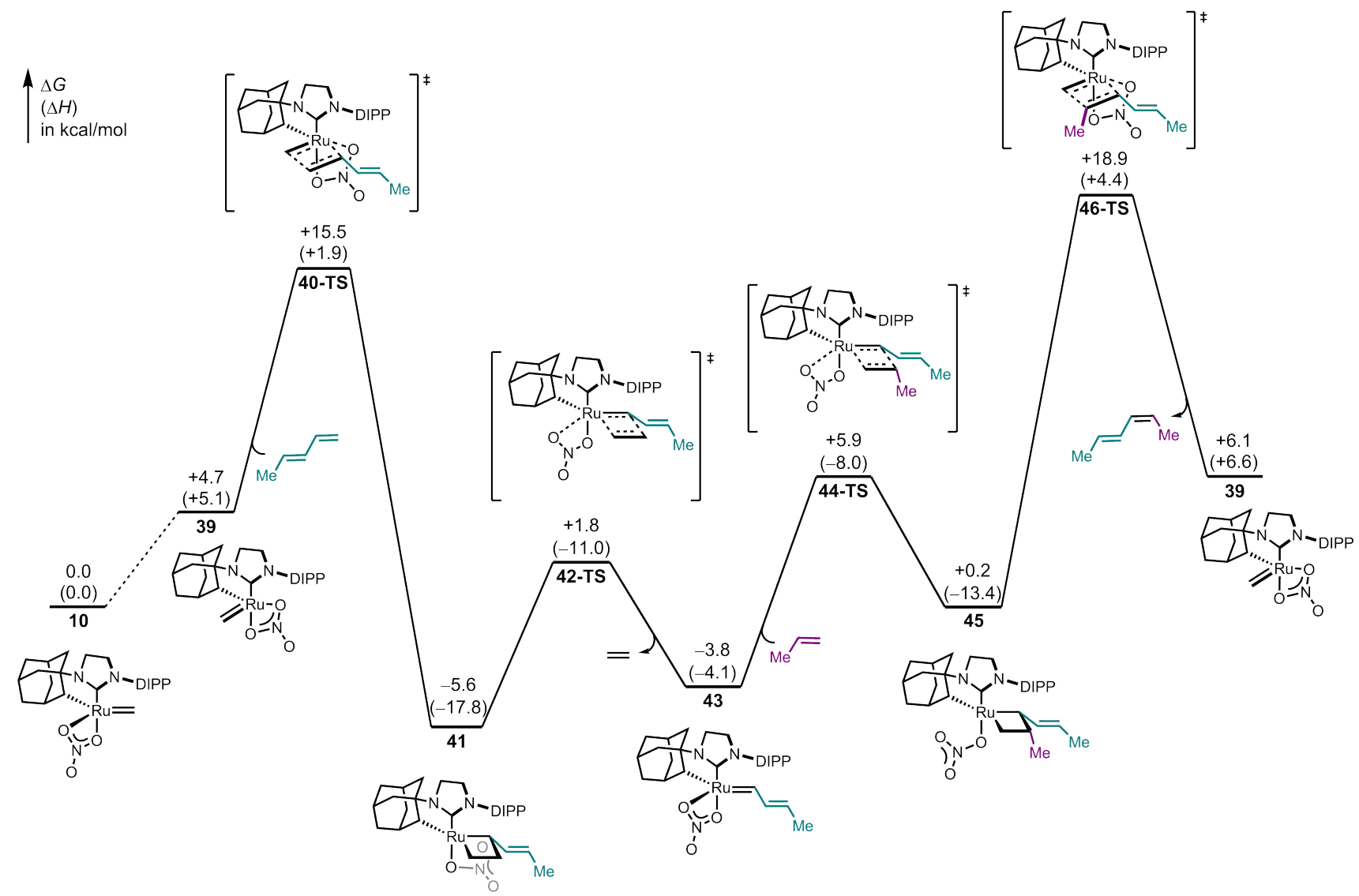

We also calculated the reaction of the diastereomer of methylidene complex 10. Methylidene 39 is about $5 \mathrm{kcal} / \mathrm{mol}$ less stable than 10, but may be accessible via non-productive metathesis pathways. Nevertheless, cross-metathesis starting from 39 is disfavored by about $7 \mathrm{kcal} / \mathrm{mol}$ in the rate-limiting transition state 46-TS (compared to 17-TS, Scheme S3). 


\section{Calculations using larger substrate models}

Scheme S6: Rate-limiting transition states using larger substrates 1-butene and E-1,3-hexadiene

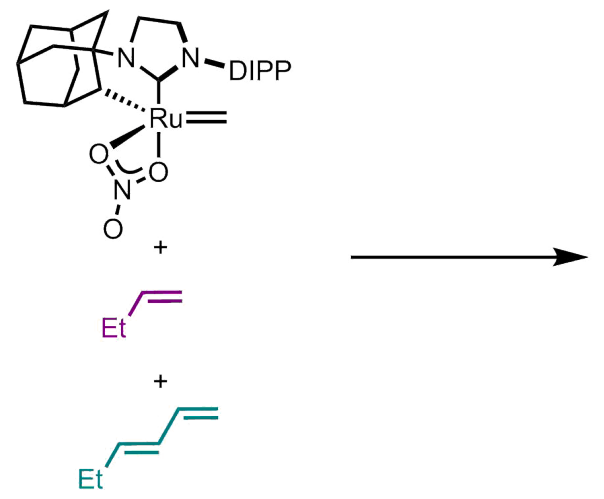

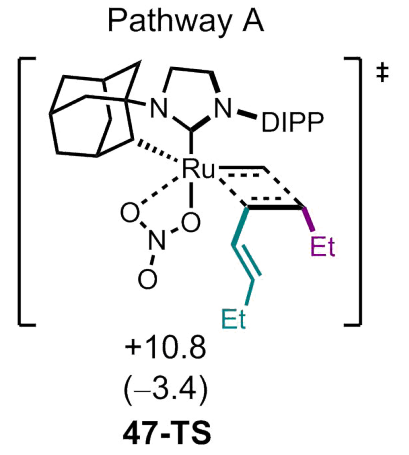

Alkene Homodimerization

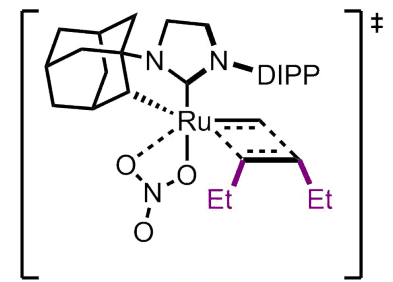

$+11.5$

$(-2.8)$

49-TS
Pathway B

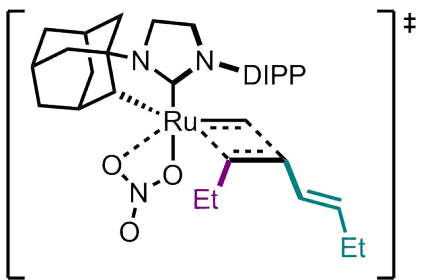

$+13.1$

$(-0.6)$

48-TS

Diene Homodimerization

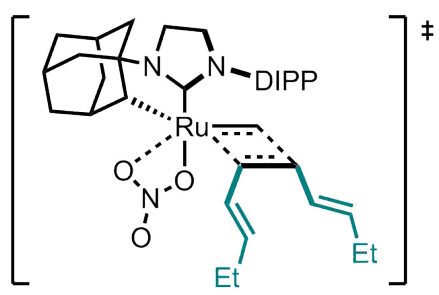

$+13.3$

$(-0.4)$

50-TS 


\section{Cartesian coordinates $(\AA)$, energies (hartree), and thermal corrections for optimized structures}

\section{Diene-First Pathway A}

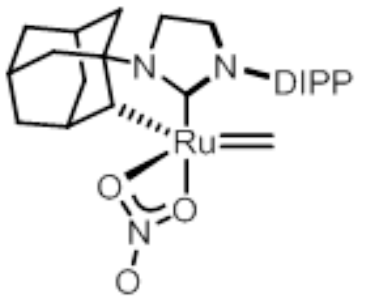

\section{0}

SCF Energy (M06, n-octanol): $\quad-1497.167761$

SCF Energy (B3LYP, gas-phase): $\quad-1496.738551$

Enthalpy Correction: $\quad 0.633713$

Free-Energy Correction: $\quad 0.542099$

\begin{tabular}{|c|c|}
\hline $\mathrm{Ru}$ & 0.49185500 \\
\hline 0 & -1.03909200 \\
\hline O & 0.78301500 \\
\hline $\mathrm{N}$ & 0.94790800 \\
\hline $\mathrm{N}$ & -1.21684100 \\
\hline C & -0.03544500 \\
\hline C & 0.47701800 \\
\hline $\mathrm{H}$ & 0.86780400 \\
\hline $\mathrm{H}$ & 0.78658400 \\
\hline C & -1.05071100 \\
\hline $\mathrm{H}$ & -1.59505700 \\
\hline $\mathrm{H}$ & -1.44201600 \\
\hline C & 2.33810800 \\
\hline C & 2.87725500 \\
\hline $\mathrm{H}$ & 2.19204600 \\
\hline $\mathrm{H}$ & 2.91303600 \\
\hline C & 4.28559600 \\
\hline $\mathrm{H}$ & 4.65338700 \\
\hline C & 5.23092000 \\
\hline $\mathrm{H}$ & 5.30623700 \\
\hline $\mathrm{H}$ & 6.24397200 \\
\hline C & 4.69743600 \\
\hline $\mathrm{H}$ & 5.36687300 \\
\hline $\mathrm{C}$ & 3.28696600 \\
\hline $\mathrm{H}$ & 2.90188600 \\
\hline $\mathrm{H}$ & 3.34399300 \\
\hline C & 4.63963700 \\
\hline $\mathrm{H}$ & 4.29887200 \\
\hline $\mathrm{H}$ & 5.64773800 \\
\hline C & 3.67851400 \\
\hline $\mathrm{H}$ & 3.63196700 \\
\hline C & 2.28965000 \\
\hline $\mathrm{H}$ & 2.03893100 \\
\hline C & 4.20502200 \\
\hline $\mathrm{H}$ & 3.54329700 \\
\hline $\mathrm{H}$ & 5.19844400 \\
\hline $\mathrm{C}$ & -2.53014400 \\
\hline C & -3.22684100 \\
\hline C & -4.52309200 \\
\hline $\mathrm{H}$ & -5.07778800 \\
\hline C & -5.11337900 \\
\hline C & -4.41588800 \\
\hline $\mathrm{H}$ & -4.88651900 \\
\hline C & -3.11827600 \\
\hline C & -2.62872000 \\
\hline $\mathrm{H}$ & -1.58422300 \\
\hline $\mathrm{C}$ & -2.41809000 \\
\hline
\end{tabular}

$$
\begin{aligned}
& 1.07399500-0.51155600 \\
& 2.59279600-0.18073100 \\
& 3.22779200-1.19104700 \\
& -1.44230800 \quad 0.83146400 \\
& -1.31659000 \quad 0.46622500 \\
& -0.66976300 \quad 0.30321100 \\
& -2.77490700 \quad 1.21970600 \\
& -3.07448600 \quad 2.19661300 \\
& -3.52759700 \quad 0.48178800 \\
& -2.57345300 \quad 1.22855600 \\
& -3.39241400 \quad 0.75016500 \\
& -2.44990200 \quad 2.24599300 \\
& -1.06350100 \quad 0.55687100 \\
& -1.81060600-0.68949400 \\
& -1.65547100-1.52979100 \\
& -2.89049000-0.48570600 \\
& -1.29482000-1.04425300 \\
& -1.83140900 \quad-1.92882900 \\
& -1.54830800 \quad 0.14719600 \\
& -2.62647600 \quad 0.34828700 \\
& -1.19690400 \quad-0.09094500 \\
& -0.80786800 \quad 1.39087600 \\
& -0.98587400 \quad 2.24260900 \\
& -1.34180500 \quad 1.74545100 \\
& -0.84750900 \quad 2.64722900 \\
& -2.41915600 \quad 1.96123800 \\
& 0.70550100 \quad 1.08474800 \\
& 1.26048700 \quad 1.96946500 \\
& 1.07273900 \quad 0.84667100 \\
& 0.96551000-0.10658800 \\
& 2.04164700-0.31326100 \\
& 0.45886200 \quad 0.30582900 \\
& 0.93761300 \quad 1.28216000 \\
& 0.21608900-1.34607800 \\
& 0.39529500-2.20193500 \\
& 0.59835200-1.61786800 \\
& \begin{array}{ll}
-0.76409400 & 0.25758800
\end{array} \\
& -1.09507700 \quad-0.92556400 \\
& -0.59232500 \quad-1.08967500 \\
& -0.83026700-1.99297300 \\
& 0.20751200-0.11613900 \\
& 0.51280400 \quad 1.04839100 \\
& 1.13520600 \quad 1.80383200 \\
& 0.03401000 \quad 1.26544400 \\
& -1.99201500-2.00438800 \\
& -2.18051600-1.73673700 \\
& 0.35784400 \quad 2.58341300
\end{aligned}
$$




$\begin{array}{lrrr}\mathrm{H} & -1.38435400 & 0.00325700 & 2.51768100 \\ \mathrm{C} & 0.58112200 & 0.37312500 & -2.19038700 \\ \mathrm{H} & 0.23554400 & -0.61167400 & -2.52170500 \\ \mathrm{H} & 0.96304600 & 1.02500800 & -2.98957700 \\ \mathrm{H} & -6.11910900 & 0.59184800 & -0.26417100 \\ \mathrm{C} & -3.34927900 & -3.35475700 & -2.06077200 \\ \mathrm{H} & -4.40262300 & -3.23502400 & -2.34059300 \\ \mathrm{H} & -2.87836600 & -4.00935900 & -2.80409200 \\ \mathrm{H} & -3.32272400 & -3.86656000 & -1.09158600 \\ \mathrm{C} & -2.63323700 & -1.31655600 & -3.38912100 \\ \mathrm{H} & -2.11918900 & -1.94999800 & -4.12227200 \\ \mathrm{H} & -3.65275000 & -1.15170600 & -3.75621600 \\ \mathrm{H} & -2.12714600 & -0.34654000 & -3.35730100 \\ \mathrm{C} & -3.09216700 & -0.38040800 & 3.75968700 \\ \mathrm{H} & -3.12092500 & -1.46485800 & 3.60100100 \\ \mathrm{H} & -2.55250400 & -0.18649100 & 4.69440900 \\ \mathrm{H} & -4.12605600 & -0.04158200 & 3.89474600 \\ \mathrm{C} & -2.35760500 & 1.87250800 & 2.86297600 \\ \mathrm{H} & -3.35720800 & 2.29043600 & 3.03087200 \\ \mathrm{H} & -1.76932800 & 2.06152500 & 3.76933200 \\ \mathrm{H} & -1.90273500 & 2.41230400 & 2.02825600 \\ \mathrm{~N} & -0.36240600 & 3.56682400 & -0.73695400 \\ \mathrm{O} & -0.80660900 & 4.69119600 & -0.79926900\end{array}$

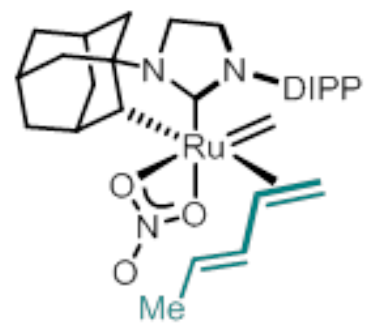

11-C

SCF Energy (M06, n-octanol): SCF Energy (B3LYP, gas-phase): Enthalpy Correction:

Free-Energy Correction:

-1692.379703
-1692.043911
0.757079
0.646719

$\mathrm{Ru}$
$\mathrm{O}$
$\mathrm{O}$
$\mathrm{N}$
$\mathrm{N}$
$\mathrm{C}$
$\mathrm{C}$
$\mathrm{H}$
$\mathrm{H}$
$\mathrm{C}$
$\mathrm{H}$
$\mathrm{H}$
$\mathrm{C}$
$\mathrm{C}$
$\mathrm{H}$
$\mathrm{H}$
$\mathrm{C}$
$\mathrm{H}$
$\mathrm{C}$
$\mathrm{H}$
$\mathrm{H}$
$\mathrm{C}$
$\mathrm{H}$
$\mathrm{C}$
$\mathrm{H}$
$\mathrm{H}$
$\mathrm{C}$

0.48882900
0.01362300
0.80676600
1.41178300
-0.73662400
0.28902000
1.21625100
1.69612300
1.62680000
-0.31655700
-0.71750700
-0.68265800
2.70330400
3.30086700
2.55951400
3.53149600
4.57938400
4.98828400
5.61367500
5.88311600
6.53745500
5.02443000
5.75644300
3.74563300
3.32810000
3.99713900
4.69517400

0.66171900

2.37823800

2.84151000

$-1.65296800$

$-1.97947000$

$-1.11279200$

$-3.03323200$

$-3.21805600$

$-3.74555000$

$-3.11568500$

$-4.05788400$

$-2.97759300$

$-1.05865600$

$-1.76722000$

$-1.77588400$

$-2.81349800$

$-1.04097600$

$-1.55185400$

$-1.06952600$

$-2.10794000$

$-0.56339700$

$-0.36772900$

$-0.38783800$

$-0.51660900$

0.93703500

$-1.03740600$

0.93005300

0.60813900

0.37735900

1.37960500

2.34503900

0.64993200

1. 45520200

1.07172300

2.48065600

0.56587000

$-0.67500000$

$-1.48026400$

$-0.42807400$

$-1.13500000$

$-2.01699600$

0.00821100

0.24886600

$-0.30291800$

1.24905600

$-1.11378900 \quad 1.70735300$

$-0.64784400 \quad 2.60956300$

$-2.15464900 \quad 1.96094000$

$1.09708900 \quad 0.88364200$

S32 


\begin{tabular}{|c|c|c|c|}
\hline $\mathrm{H}$ & 4.31434500 & 1.63457600 & 1.76214300 \\
\hline $\mathrm{H}$ & 5.61275100 & 1.61215500 & 0.56608700 \\
\hline $\mathrm{C}$ & 3.64129600 & 1.13083800 & -0.25667100 \\
\hline $\mathrm{H}$ & 3.39958400 & 2.16979500 & -0.50601600 \\
\hline $\mathrm{C}$ & 2.38863900 & 0.42275200 & 0.27955400 \\
\hline $\mathrm{H}$ & 2.12295000 & 0.89199200 & 1.24352900 \\
\hline C & 4.22293700 & 0.41654800 & -1.49268500 \\
\hline $\mathrm{H}$ & 3.49865700 & 0.43524400 & -2.31588300 \\
\hline $\mathrm{H}$ & 5.11896200 & 0.94910900 & -1.83983600 \\
\hline $\mathrm{C}$ & -2.13284100 & -1.73773500 & 0.37156200 \\
\hline C & -2.73833300 & -2.32181200 & -0.76547000 \\
\hline C & -4.10867900 & -2.11222500 & -0.96451600 \\
\hline $\mathrm{H}$ & -4.59521700 & -2.54729100 & -1.83299000 \\
\hline C & -4.85979600 & -1.36092200 & -0.06497500 \\
\hline $\mathrm{C}$ & -4.25257900 & -0.81776800 & 1.06368100 \\
\hline $\mathrm{H}$ & -4.85198000 & -0.25214300 & 1.77143700 \\
\hline C & -2.88520000 & -0.99717600 & 1.31270200 \\
\hline C & -1.96395500 & -3.20368500 & -1.74215900 \\
\hline $\mathrm{H}$ & -0.90443400 & -3.14282300 & -1.47496300 \\
\hline C & -2.28446800 & -0.45486400 & 2.60836000 \\
\hline $\mathrm{H}$ & -1.19962200 & -0.58853500 & 2.56410200 \\
\hline C & 0.73932000 & -0.12039100 & -2.16782600 \\
\hline $\mathrm{H}$ & 0.52280000 & -1.14229900 & -2.50995500 \\
\hline $\mathrm{H}$ & 1.07853500 & 0.52842500 & -2.99220300 \\
\hline $\mathrm{C}$ & -2.15747800 & 1.30840800 & -2.12054600 \\
\hline $\mathrm{H}$ & -1.58931400 & 1.68770600 & -2.96603100 \\
\hline $\mathrm{H}$ & -2.38552400 & 0.24930100 & -2.10846700 \\
\hline $\mathrm{C}$ & -2.63174100 & 2.13720400 & -1.16803300 \\
\hline $\mathrm{H}$ & -3.21687000 & 1.71695700 & -0.35075400 \\
\hline C & -2.42967300 & 3.57569400 & -1.15063400 \\
\hline $\mathrm{H}$ & -1.85026100 & 4.00135500 & -1.97001300 \\
\hline C & -2.88444400 & 4.38019800 & -0.17443500 \\
\hline $\mathrm{H}$ & -3.44654700 & 3.93389900 & 0.64788100 \\
\hline $\mathrm{C}$ & -2.64400500 & 5.85547200 & -0.09135300 \\
\hline $\mathrm{H}$ & -3.58460400 & 6.41033600 & 0.02861000 \\
\hline$H$ & -2.01718700 & 6.09014600 & 0.77939100 \\
\hline $\mathrm{H}$ & -2.12965300 & 6.23040300 & -0.98177100 \\
\hline $\mathrm{N}$ & 0.44073600 & 3.27753000 & 0.11357800 \\
\hline O & 0.49887900 & 4.45575300 & 0.41461500 \\
\hline $\mathrm{C}$ & -2.09444800 & -2.73364800 & -3.20341300 \\
\hline $\mathrm{H}$ & -1.48301900 & -3.36549000 & -3.85864100 \\
\hline $\mathrm{H}$ & -3.12861300 & -2.79488300 & -3.56121800 \\
\hline H & -1.75853400 & -1.69882700 & -3.32244700 \\
\hline C & -2.39765400 & -4.67896100 & -1.61181200 \\
\hline $\mathrm{H}$ & -3.44930600 & -4.80857700 & -1.89390600 \\
\hline $\mathrm{H}$ & -1.79413100 & -5.31700600 & -2.26845800 \\
\hline $\mathrm{H}$ & -2.28535600 & -5.04484400 & -0.58473900 \\
\hline $\mathrm{C}$ & -2.81005000 & -1.25597100 & 3.81966000 \\
\hline $\mathrm{H}$ & -2.32391600 & -0.91650800 & 4.74184500 \\
\hline $\mathrm{H}$ & -3.89122300 & -1.12073700 & 3.94280000 \\
\hline $\mathrm{H}$ & -2.62434600 & -2.33098100 & 3.71142000 \\
\hline C & -2.53887100 & 1.05097800 & 2.80955100 \\
\hline $\mathrm{H}$ & -3.61048000 & 1.27622000 & 2.87257300 \\
\hline $\mathrm{H}$ & -2.08015800 & 1.38119600 & 3.74883600 \\
\hline $\mathrm{H}$ & -2.09964500 & 1.64418600 & 2.00352000 \\
\hline $\mathrm{H}$ & -5.92178100 & -1.20787700 & -0.23799500 \\
\hline
\end{tabular}




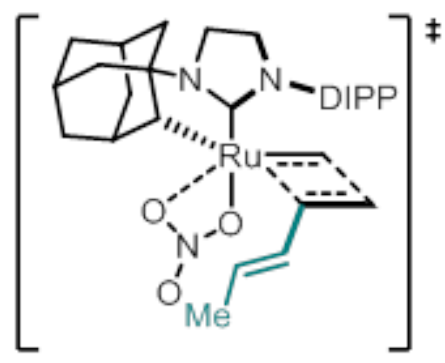

\section{1-TS}

SCF Energy (M06, n-octanol): $\quad-1692.376027$

SCF Energy (B3LYP, gas-phase): $\quad-1692.037055$

Enthalpy Correction:

0.757467

Free-Energy Correction:

0.654734

\begin{tabular}{|c|c|c|c|}
\hline $\mathrm{Ru}$ & 0.61246000 & 0.64556400 & -0.59162700 \\
\hline 0 & 1.71661300 & 2.52825500 & -1.06588500 \\
\hline 0 & 1.00811400 & 2.35860000 & 0.98283000 \\
\hline $\mathrm{N}$ & 0.81638900 & -1.95868700 & 0.64653000 \\
\hline $\mathrm{N}$ & -1.34193300 & -1.67266000 & 0.38548600 \\
\hline C & -0.11661300 & -1.07931000 & 0.19887300 \\
\hline $\mathrm{C}$ & 0.27188100 & -3.27738600 & 0.96896600 \\
\hline $\mathrm{H}$ & 0.70816400 & -3.68378100 & 1.88558800 \\
\hline $\mathrm{H}$ & 0.46307300 & -3.98840500 & 0.15242400 \\
\hline $\mathrm{C}$ & -1.21915200 & -2.95744900 & 1.11238500 \\
\hline $\mathrm{H}$ & -1.86747300 & -3.71978200 & 0.67435900 \\
\hline $\mathrm{H}$ & -1.50862600 & -2.82214600 & 2.16267600 \\
\hline C & 2.23453100 & -1.66914200 & 0.40920900 \\
\hline $\mathrm{C}$ & 2.71664700 & -2.30084200 & -0.92011600 \\
\hline $\mathrm{H}$ & 2.05301400 & -1.98734000 & -1.73234200 \\
\hline $\mathrm{H}$ & 2.66218500 & -3.39746500 & -0.85226200 \\
\hline $\mathrm{C}$ & 4.16449700 & -1.86048600 & -1.21193100 \\
\hline $\mathrm{H}$ & 4.49242500 & -2.30230100 & -2.16260500 \\
\hline $\mathrm{C}$ & 5.07877800 & -2.34924300 & -0.07033700 \\
\hline $\mathrm{H}$ & 5.06328700 & -3.44745100 & -0.01647300 \\
\hline $\mathrm{H}$ & 6.11884800 & -2.05383100 & -0.26431700 \\
\hline $\mathrm{C}$ & 4.60133200 & -1.74002600 & 1.26402200 \\
\hline $\mathrm{H}$ & 5.24449900 & -2.09202300 & 2.08161700 \\
\hline C & 3.14651400 & -2.19157300 & 1.54611600 \\
\hline $\mathrm{H}$ & 2.79938600 & -1.79262900 & 2.50830600 \\
\hline $\mathrm{H}$ & 3.11090700 & -3.28976200 & 1.61133900 \\
\hline $\mathrm{C}$ & 4.67696500 & -0.20117100 & 1.16582600 \\
\hline $\mathrm{H}$ & 4.38097500 & 0.25790800 & 2.11844700 \\
\hline $\mathrm{H}$ & 5.71537800 & 0.10407500 & 0.97259200 \\
\hline $\mathrm{C}$ & 3.74700300 & 0.29904200 & 0.02907800 \\
\hline $\mathrm{H}$ & 3.81030900 & 1.38962500 & -0.03685700 \\
\hline $\mathrm{C}$ & 2.31385800 & -0.13039700 & 0.38785300 \\
\hline $\mathrm{H}$ & 2.10774100 & 0.20958300 & 1.41290900 \\
\hline $\mathrm{C}$ & 4.21043600 & -0.32081200 & -1.30467800 \\
\hline $\mathrm{H}$ & 3.56732000 & 0.02822400 & -2.12192000 \\
\hline $\mathrm{H}$ & 5.23307300 & 0.00890500 & -1.53476700 \\
\hline $\mathrm{C}$ & -2.62483600 & -1.02298400 & 0.38418500 \\
\hline $\mathrm{C}$ & -3.54587400 & -1.33562000 & -0.64539500 \\
\hline $\mathrm{C}$ & -4.79934100 & -0.71169300 & -0.62324800 \\
\hline $\mathrm{H}$ & -5.51940300 & -0.93043700 & -1.40651700 \\
\hline $\mathrm{C}$ & -5.14243800 & 0.17947800 & 0.38966300 \\
\hline $\mathrm{C}$ & -4.24489500 & 0.43585100 & 1.42162700 \\
\hline $\mathrm{H}$ & -4.53592300 & 1.10479300 & 2.22641500 \\
\hline $\mathrm{C}$ & -2.97972100 & -0.16648300 & 1.45498300 \\
\hline $\mathrm{C}$ & -3.24132700 & -2.37762000 & -1.72139800 \\
\hline $\mathrm{H}$ & -2.16883700 & -2.59374100 & -1.67562000 \\
\hline $\mathrm{C}$ & -2.08641300 & 0.05208300 & 2.67729400 \\
\hline $\mathrm{H}$ & -1.11886400 & -0.41990300 & 2.48678200 \\
\hline $\mathrm{C}$ & 0.49000700 & -0.03119400 & -2.34833300 \\
\hline $\mathrm{H}$ & 0.01321900 & -0.94492200 & -2.72428100 \\
\hline $\mathrm{C}$ & -1.30790400 & 1.98580500 & -0.98064400 \\
\hline $\mathrm{H}$ & -1.90178100 & 1.68559600 & -0.12344500 \\
\hline
\end{tabular}

\section{S34}




$\begin{array}{rrr}-1.26963500 & 1.10363500 & -2.08420600 \\ -1.92059200 & 0.24150700 & -2.05875200 \\ 1.00811500 & 0.51964300 & -3.14593900 \\ 1.63811500 & 3.05191200 & 0.10930100 \\ 2.13533000 & 4.13722200 & 0.36453700 \\ -3.55893700 & -1.90479700 & -3.15317400 \\ -3.26233900 & -2.67572500 & -3.87385600 \\ -4.63037400 & -1.72278500 & -3.29449400 \\ -3.02842300 & -0.98330900 & -3.41209600 \\ -4.00455300 & -3.68923500 & -1.43190900 \\ -3.72632800 & -4.46493300 & -2.15532800 \\ -3.79699000 & -4.07068500 & -0.42636900 \\ -5.08753800 & -3.53416800 & -1.50573800 \\ -2.70894400 & -0.63343500 & 3.91420600 \\ -3.66008000 & -0.16276500 & 4.18959200 \\ -2.90815000 & -1.69647900 & 3.73556900 \\ -2.03430600 & -0.55197000 & 4.77442100 \\ -1.80369700 & 1.53619800 & 2.97807600 \\ -1.25131900 & 2.01684300 & 2.16666400 \\ -2.72767600 & 2.09858000 & 3.15927200 \\ -1.18874900 & 1.61865900 & 3.88165900 \\ -6.11775900 & 0.65884600 & 0.38514600 \\ -1.03346300 & 3.40936000 & -1.12905300 \\ -0.56638400 & 3.72430800 & -2.06168400 \\ -1.30555600 & 4.33091300 & -0.18869700 \\ -1.75069200 & 4.00277700 & 0.75090000 \\ -1.00042700 & 5.79217100 & -0.30769700 \\ -0.24103400 & 6.08214400 & 0.43094800 \\ -0.61338900 & 6.04373200 & -1.30056300 \\ -1.88955500 & 6.40784100 & -0.11475600 \\ -1.09835800 & 1.54194900 & -3.06537600\end{array}$

$12 a$

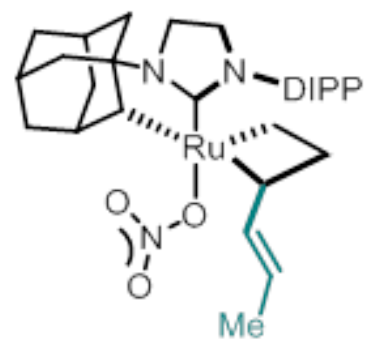

SCF Energy (M06, n-octanol):

$-1692.397781$

SCF Energy (B3LYP, gas-phase):

$-1692.051345$

Enthalpy Correction:

0.760023

Free-Energy Correction:

0.654882

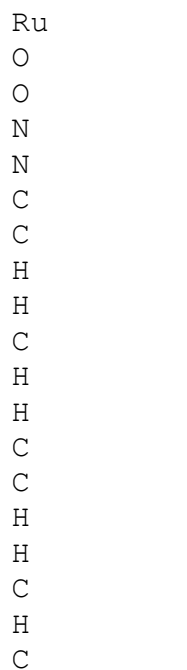

$$
\begin{array}{r}
0.64706100 \\
2.72939800 \\
2.28692700 \\
-1.99446700 \\
-1.64856800 \\
-1.06505000 \\
-3.33166100 \\
-3.82349500 \\
-3.96774100 \\
-3.00858900 \\
-3.70665300 \\
-2.98918300 \\
-1.70521500 \\
-2.20191400 \\
-1.77914100 \\
-3.29649100 \\
-1.78038900 \\
-2.13793300 \\
-2.40413100
\end{array}
$$

\section{S35}

$-0.60045700$

$-1.45913000$

0.56844900

0.21748700

0.14231900

0.73344900

1.63676200

$-0.12921600$

0.80223800

0.23272100

1.83569100

0.43040700

$-0.93617100$

$-1.74328700$

$-0.99467000$

$-1.10097200$

$-2.07056800$

0.03651400 


$$
\begin{aligned}
& \begin{array}{llll}
4.95068900 & -3.50145200 & -0.01784700
\end{array} \\
& 6.05013300-2.12083600-0.07137100 \\
& 4.45709900-1.91509100 \quad 1.39779100 \\
& 5.04875400 \quad-2.35820100 \quad 2.20973500 \\
& 2.98350400 \quad-2.35359100 \quad 1.55695900 \\
& 2.58995200 \quad-2.04093600 \quad 2.53323700 \\
& 2.91690300-3.45095700 \quad 1.51012100 \\
& 4.55589800 \quad-0.37729900 \quad 1.45756800 \\
& \begin{array}{lll}
4.20644500 & -0.00817500 & 2.43096200
\end{array} \\
& 5.60562100-0.06768400 \quad 1.35698800 \\
& \begin{array}{lll}
3.71166000 & 0.25529100 & 0.31792300
\end{array} \\
& \begin{array}{lll}
3.79836500 & 1.34115200 & 0.38460800
\end{array} \\
& 2.25091500-0.17141700 \quad 0.53948800 \\
& \begin{array}{lll}
1.93013400 & 0.13462800 & 1.54642300
\end{array} \\
& \begin{array}{llll}
4.24980300 & -0.24210900 & -1.03895000
\end{array} \\
& 3.67758400 \quad 0.21237200 \quad-1.85661500 \\
& \begin{array}{lll}
5.29363400 & 0.07671600 & -1.16377300
\end{array} \\
& \begin{array}{llr}
-2.68310000 & -0.96976100 & 0.28418900
\end{array} \\
& -3.58831300-1.09619400-0.79519500 \\
& \begin{array}{lll}
-4.81839000 & -0.43185900 & -0.70407500
\end{array} \\
& -5.52729900 \quad-0.50504500 \quad-1.52368400 \\
& \begin{array}{lll}
-5.15473800 & 0.31139800 & 0.42367400
\end{array} \\
& \begin{array}{lll}
-4.27575500 & 0.37489600 & 1.50154000
\end{array} \\
& \begin{array}{lll}
-4.56263200 & 0.92802200 & 2.39152200
\end{array} \\
& -3.03395900 \\
& -3.30150600 \\
& -2.24009500 \\
& -2.14746000 \\
& -1.20901000 \\
& 0.03252200 \\
& -1.01054400 \\
& -0.96565800 \\
& -0.47035100 \\
& 0.74274400 \\
& -1.97804000 \\
& -0.70833700 \\
& -1.84035600 \\
& -0.90785500 \\
& -0.17409100 \\
& -1.69502900 \\
& -2.44917700 \\
& -1.61712800 \\
& -2.57548200 \\
& -1.38614500 \\
& -0.84204200 \\
& -6.11172000 \\
& -2.82289900 \\
& -3.74901500 \\
& -3.07912900 \\
& -2.15488600 \\
& -1.77345300 \\
& -2.66144400 \\
& -1.14000900 \\
& -1.21958700 \\
& -4.12065500 \\
& -5.19583000 \\
& -3.86283000 \\
& -3.94093100 \\
& -3.56817900 \\
& -2.97855400 \\
& -3.30265800 \\
& -4.62494500 \\
& \text { 2. } 18803400
\end{aligned}
$$

\section{S36}




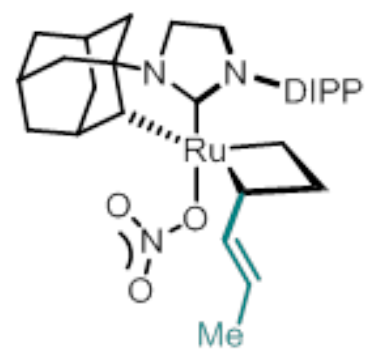

$12 b$

SCF Energy (M06, n-octanol): $-1692.400995$ SCF Energy (B3LYP, gas-phase): Enthalpy Correction:

Free-Energy Correction:
$-1692.048723$

0.759859

0.654990

$$
\begin{aligned}
& 0.61553500 \\
& \text { 2. } 77878000 \\
& 1.93113600 \\
& -1.50149600 \\
& -1.07298200 \\
& -0.74217800 \\
& -2.53521300 \\
& -2.63878600 \\
& -3.51083600 \\
& -1.97914200 \\
& -2.74532400 \\
& -1.40636800 \\
& -1.60091400 \\
& -2.72835500 \\
& -2.54585600 \\
& -3.69948000 \\
& -2.75636700 \\
& -3.56483000 \\
& -3.00454200 \\
& -3.98214400 \\
& -3.03466100 \\
& -1.87901500 \\
& -2.05283800 \\
& -1.87466600 \\
& -1.09856800 \\
& -2.84343900 \\
& -0.52567000 \\
& 0.29271500 \\
& -0.53113900 \\
& -0.26963200 \\
& 0.69711200 \\
& -0.24081900 \\
& 0.54200900 \\
& -1.39911000 \\
& -1.22948100 \\
& -1.39739000 \\
& -0.74303900 \\
& -1.47695400 \\
& -1.15488900 \\
& -1.68759300 \\
& -0.17408900 \\
& 0.48975100 \\
& \text { 1. } 23013400 \\
& 0.21795400 \\
& -2.65610800 \\
& -2.50366000 \\
& 0.90813200 \\
& 0.77910900
\end{aligned}
$$




$\begin{array}{rrr}-0.35008000 & 0.42306300 & -2.29451300 \\ -1.03344000 & -0.40713400 & -2.44019600 \\ -0.65540300 & 2.09758300 & -0.24039200 \\ -1.40670100 & 1.80190000 & 0.48796100 \\ -1.05832200 & 1.71094200 & -1.71709500 \\ -2.13309300 & 1.51466000 & -1.70379000 \\ 0.25319300 & 0.64258600 & -3.17855800 \\ -0.16069700 & 3.46386800 & -0.02200400 \\ 0.39303100 & 3.92772300 & -0.83568500 \\ -0.34742100 & 4.15523500 & 1.11557200 \\ -0.89958600 & 3.68655000 & 1.93238100 \\ 0.18136300 & 5.53352000 & 1.37537200 \\ 0.88004600 & 5.52980400 & 2.22343800 \\ 0.71470300 & 5.92848500 & 0.50518100 \\ -0.62608900 & 6.23226600 & 1.63554200 \\ -4.39514000 & 0.24149900 & 3.03121400 \\ -4.20219400 & 0.69354100 & 4.01141500 \\ -5.45953200 & 0.37349600 & 2.80389900 \\ -4.20800900 & -0.83450400 & 3.10901500 \\ -3.80924000 & 2.42186700 & 1.91363500 \\ -4.87355200 & 2.62869700 & 1.75577700 \\ -3.53377800 & 2.87167900 & 2.87457100 \\ -3.24634700 & 2.93382900 & 1.12780800 \\ -2.80687700 & -3.97743600 & -0.96661600 \\ -2.80737600 & -3.95840500 & 0.12751000 \\ -3.83470800 & -4.17453900 & -1.29357600 \\ -2.18286100 & -4.81968400 & -1.28851600 \\ -2.22754100 & -2.80824300 & -3.11003600 \\ -1.48290200 & -3.56731300 & -3.37435100 \\ -3.18590400 & -3.14121600 & -3.52483200 \\ -1.94998300 & -1.87668400 & -3.61203500 \\ -6.08811000 & 0.05835600 & -1.86857200 \\ -0.81596800 & 2.55643200 & -2.36329300 \\ 2.69118300 & 2.81279400 & -1.28088000 \\ 3.24677200 & 3.63468900 & -2.00877000\end{array}$

$12 \mathrm{C}$

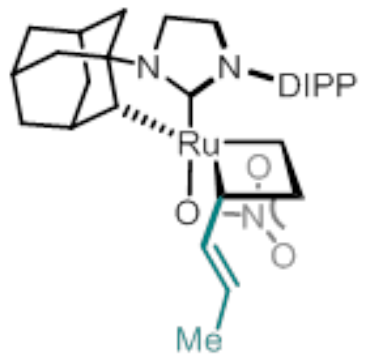

SCF Energy (M06, n-octanol): $\quad-1692.396138$

SCF Energy (B3LYP, gas-phase): $\quad-1692.052351$

Enthalpy Correction:

0.759864

Free-Energy Correction:

0.655095

$\begin{array}{lrrr}\text { Ru } & -0.60364000 & 0.73308600 & 0.62143300 \\ \mathrm{O} & -2.09621400 & -0.00380700 & 2.66298700 \\ \mathrm{O} & -1.82888700 & 2.02931500 & 1.91453000 \\ \mathrm{~N} & -0.44247100 & -1.18745200 & -1.54193100 \\ \mathrm{~N} & 1.64709900 & -0.91983200 & -0.93430500 \\ \mathrm{C} & 0.35969600 & -0.55178900 & -0.64456100 \\ \mathrm{C} & 0.25981800 & -2.16206300 & -2.38072000 \\ \mathrm{H} & -0.03191200 & -2.07110900 & -3.43076300 \\ \mathrm{H} & 0.04426100 & -3.18800600 & -2.05313000 \\ \mathrm{C} & 1.72367400 & -1.77334100 & -2.14241800 \\ \mathrm{H} & 2.37402200 & -2.63361800 & -1.96589500 \\ \mathrm{H} & 2.13533000 & -1.20045300 & -2.98195100 \\ \mathrm{C} & -1.88851900 & -1.21471200 & -1.31438400 \\ \mathrm{C} & -2.30044800 & -2.50887500 & -0.56324700\end{array}$

\section{S38}


$-1.72613900$

$-2.05377300$

$-3.81121600$

$-4.08509400$

$-4.60399600$

$-4.40977400$

$-5.68158800$

$-4.20103600$

$-4.76850100$

$-2.69275300$

$-2.38400600$

$-2.48507700$

$-4.49859300$

$-4.24609200$

$-5.57280400$

$-3.69004400$

$-3.90420800$

$-2.20703000$

$-2.00142000$

$-4.11276100$

$-3.58435900$

$-5.18761500$

2. 83177400

3.04726900

4.22393600

4.40411400

5.17300100

4.97236300

5.73384200

3.81183700

2.09401200

1.15906500

3.68839000

2.65318200

0.73418800

1. 50487600

0.54587500

1.26234200

1.18576000

2.26746200

0.26427200

$-0.15905500$

$-0.79997400$

$-0.08372400$

0.55982700

$-0.82390000$

$-1.48317300$

$-1.44042000$

$-0.13253300$

4.60428900

4.46510100

5.65931200

4.40156100

3. 99842000

5.04790700

3. 81368900

3. 37646400

2. 68945800

2. 92640800

3. 61651200

1. 98296700

1.73341500

1.02073700
$-2.57720800$

$-3.38846100$

$-2.48707500$

$-3.41722500$

$-2.36892300$

$-3.23865600$

$-2.36196200$

$-1.06944100$

$-0.97630900$

$-1.12170700$

$-0.22427800$

$-1.99022300$

0.14103000

1.07742200

0.18094100

0.01996300

0.88823600

0.01224400

0.93021600

$-1.27950400$

$-1.36559700$

$-1.23764100$

$-0.76395300$

$-1.65825500$

$-1.51829900$

$-2.18404300$

$-0.55160900$

0.27933900

1.00752000

0.18595300

$-2.81062800$

$-2.63910000$

1.04383900

0.97400600

0.68856700

$-0.07428500$

2.28067500

2.08870600

2.02001800

1.97471800

0.82512000

3.56487500

3.88772300

4.31621100

3.98308100

5.60334400

5.53989400

5.86435700

6.43637000

0.49589700

1.06702100

0.57527600

$-0.55864800$

2.53366400

2.69075000

3.10595700

2.95899600

$-4.14619300$

$-4.11718300$

$-4.38573700$

$-4.96741300$

$-2.92347100$

$-3.74326000$
0.36914000

$-1.17421800$

$-0.25732600$

0.25757200

$-1.57491700$

$-2.21897600$

$-1.36371700$

$-2.30146700$

$-3.23674700$

$-2.63479700$

$-3.18721600$

$-3.27716200$

$-1.39020400$

$-1.90614100$

$-1.16293200$

$-0.06721600$

0.56337900

$-0.45671300$

$-1.06155300$

0.64886400

1.60166600

0.87266000

$-0.13148500$

0.94814200

1.69419300

2. 53288800

1. 37418000

0.27688200

0.01386700

$-0.50337700$

1.27011800

0.73004400

$-1.76099100$

$-2.11167000$

2.14049700

2.15704900

0.02462200

$-0.77366300$

1.46964600

1.31611700

3. 11377100

$-0.15321300$

0.66693200

$-1.26489400$

$-2.08244600$

$-1.48606000$

$-2.36337100$

$-0.61944300$

$-1.67630300$

$-2.87820800$

$-3.80392500$

$-2.59048900$

$-3.09402500$

$-1.52030500$

$-1.24648900$

$-2.43687300$

$-0.72716500$

0.77146500

$-0.29820000$

1. 30578600

0.94052200

2.76359700

2.90937500 


$\begin{array}{rrrr}\mathrm{H} & 2.61080700 & -3.14185600 & 3.38340400 \\ \mathrm{H} & 1.26831400 & -2.00843800 & 3.13981100 \\ \mathrm{H} & 6.07797900 & -0.45936300 & 1.96874900 \\ \mathrm{H} & 0.91923100 & 2.86194900 & 2.11128700 \\ \mathrm{~N} & -2.37826100 & 1.22179200 & 2.77607600 \\ \mathrm{O} & -3.12741400 & 1.66446800 & 3.63676900\end{array}$

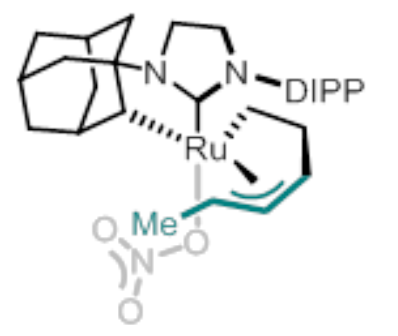

$12 d$

SCF Energy (M06, n-octanol):

$-1692.401982$

SCF Energy (B3LYP, gas-phase):

Enthalpy Correction:

$-1692.059214$

Free-Energy Correction:

0.760995

0.657941

$-0.92976900$

$-1.67555300$

$-0.75812200$

1.39588500

0.15281000

$-0.18919400$

$-0.61158700$

$-0.38241700$

1.29703200

1.94535600

1.60769500

$-2.18961500$

$-2.78313800$

$-2.19688300$

$-2.71948900$

$-4.25857300$

$-4.65934300$

$-5.05816600$

$-5.01363900$

$-6.11707200$

$-4.47575300$

$-5.03722600$

$-2.99535700$

$-2.56789800$

$-2.94166800$

$-4.59375100$

$-4.23895900$

$-5.65115700$

$-3.76621200$

$-3.84375800$

$-2.30570500$

$-2.11396900$

$-4.35414200$

$-3.84105000$

$-5.40809500$

2.66696800

3. 65111200

4.89395300

5.65723700

5.17216800

4.21595500

4.45424000

2. 95711500

3. 42732600
2.50152100

2.79193900

$-1.74368900$

$-1.42210400$

$-0.86340000$

$-3.03189300$

$-3.38976000$

$-3.79015900$

$-2.68908900$

$-3.45494300$

$-2.52629900$

$-1.51077000$

$-2.44412700$

$-2.37739000$

$-3.48753300$

$-2.07958200$

$-2.73363700$

$-2.28296400$

$-3.33627300$

$-2.04244600$

$-1.37221800$

$-1.51459100$

$-1.75723100$

$-1.15582600$

$-2.81284400$

0.10024900

0.77662600

0.34273100

0.31433200

1.35996800

$-0.01626400$

0.51208100

$-0.60570800$

$-0.45973800$

$-0.34188000$

$-0.74680400$

$-1.03811400$

$-0.40042300$

$-0.60136500$

0.47953300

0.71003100

1. 36926200

0.09487800

$-2.06695800$
$-1.06173300$

0.96135400

$-0.69075800$

$-0.42727500$

$-0.19710300$

$-1.08401800$

$-2.02742900$

$-0.31291700$

$-1.18854000$

$-0.75588600$

$-2.22769500$

$-0.51468400$

0.57193000

1. 49260700

0.23008200

0.83013700

1. 61607600

$-0.47233200$

$-0.78487500$

$-0.30777300$

$-1.57240200$

$-2.50524300$

$-1.82020800$

$-2.63252700$

$-2.12735800$

$-1.11527200$

$-1.90343000$

$-0.93680600$

0.18387200

0.50025700

$-0.18485000$

$-1.13269200$

1.27526100

2. 22966000

1. 44181100

$-0.49532600$

0.48139400

0.38239400

1.12829700

$-0.65904400$

$-1.64300600$

$-2.47266400$

$-1.59805800$

1.58961800 


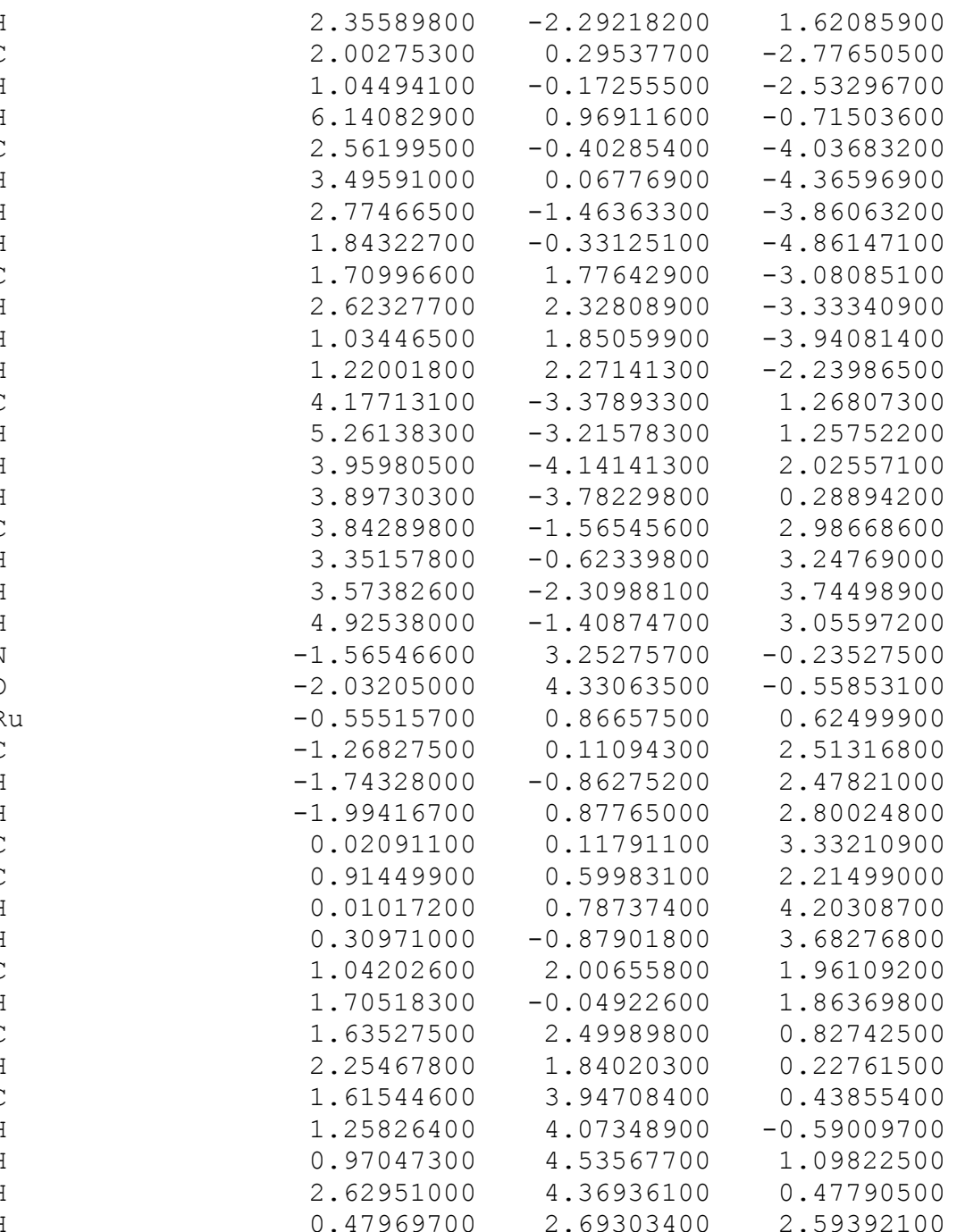

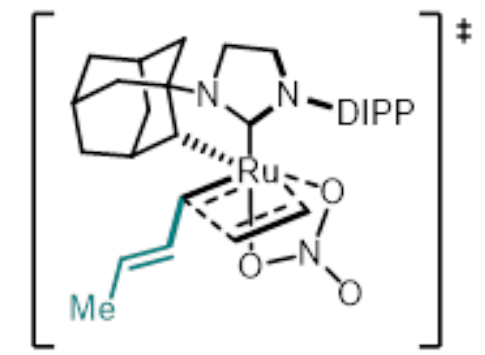

\section{3-TS}

SCF Energy (M06, n-octanol): $\quad-1692.378773$

SCF Energy (B3LYP, gas-phase): Enthalpy Correction:

Free-Energy Correction:

-1692.378773
-1692.040871
0.758122
0.653919

$\begin{array}{lr}\mathrm{Ru} & -0.55369000 \\ \mathrm{O} & -1.77180500 \\ \mathrm{O} & -1.81711900 \\ \mathrm{~N} & -0.48909000 \\ \mathrm{~N} & 1.61608300 \\ \mathrm{C} & 0.33597100 \\ \mathrm{C} & 0.18266400 \\ \mathrm{H} & -0.12029400 \\ \mathrm{H} & -0.05437300\end{array}$

0.72226900

$-0.29984000$

1.84549800

$-1.13030600$

$-0.90641900$

$-0.52989800$

$-2.09290100$

$-1.96149600$

$-3.12375400$
0.66808800

2. 46559100

2.11576900

$-1.56172300$

$-0.99017400$

$-0.66590300$

$-2.43812300$

$-3.48059800$

$-2.14184600$

\section{S41}




$\begin{array}{rrrr}\mathrm{H} & 3.01578400 & -4.15238500 & -0.44674100 \\ \mathrm{H} & 3.66283800 & -4.44111600 & 1.17180400 \\ \mathrm{H} & 2.05045300 & -5.04075300 & 0.74787700 \\ \mathrm{C} & 1.72252700 & -3.02899500 & 2.60164000 \\ \mathrm{H} & 1.05008900 & -3.88546300 & 2.72594700 \\ \mathrm{H} & 2.59449000 & -3.20275200 & 3.24293000 \\ \mathrm{H} & 1.19816900 & -2.14288200 & 2.96905300 \\ \mathrm{H} & 6.08733200 & -0.52920500 & 1.85898600 \\ \mathrm{H} & 0.97007200 & 2.83797300 & 2.08375800 \\ \mathrm{~N} & -2.23806500 & 0.83869800 & 2.80909300 \\ \mathrm{O} & -3.02952000 & 0.97998500 & 3.72708900\end{array}$

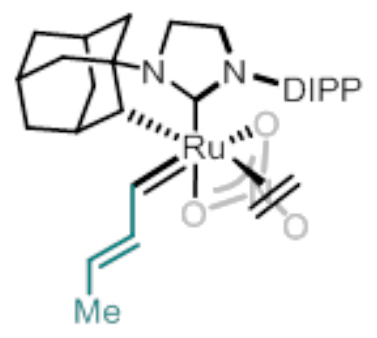

13-C

SCF Energy (M06, n-octanol):

$-1692.378784$

SCF Energy (B3LYP, gas-phase):

$-1692.045588$

Enthalpy Correction:

0.759023

Free-Energy Correction:

0.649855

Ru
O
O
N
N
C
C
H
H
C
H
H
C
C
H
H
C
H
C
H
H
C
H
C
H
H
C
H
H
C
H
C
H
C
H
H
C
C
C

-0.50754100
-1.39359700
-1.74909600
-0.55612600
1.55701900
0.30153100
0.07361400
-0.24005400
-0.18764100
1.56381100
2.17227600
1.98792100
-1.98935300
-2.35526800
-1.72397300
-2.15841400
-3.84222600
-4.08813300
-4.72166400
-4.57571200
-5.78309700
-4.35898000
-4.98896300
-2.87600100
-2.59727700
-2.73096600
-4.59227700
-4.38494400
-5.64987500
-3.68279300
-3.84976900
-2.22609200
-2.18121000
-4.07211600
-3.49200700
-5.13093300
2.78334200
3.16298800
4.37528900
0.68887000

$-0.48416700$

1.65258100

$-1.09664800$

$-0.96950300$

$-0.54124300$

$-2.09681100$

$-1.97813300$

$-3.11449400$

$-1.79302500$

$-2.69126000$

$-1.22047900$

$-1.08947100$

$-2.31575900$

$-2.32187000$

$-3.24510700$

$-2.25271500$

$-3.13491300$

$-2.23010200$

$-3.14968900$

$-2.19537600$

$-0.99532300$

$-0.96577700$

$-1.10349000$

$-0.26416900$

$-2.02950500$

0.28244900

1.17691700

0.33518500

0.26328400

1.17670000

0.23541300

1.01392200

$-0.97285000$

$-1.02669400$

$-0.89850300$

$-0.78610400$

$-1.76898100$

$-1.59500400$
0.63996800

2. 44933000

2.25855200

$-1.64918100$

$-1.08427300$

$-0.74981300$

$-2.51617300$

$-3.55705100$

$-2.19477600$

$-2.31074900$

$-2.17171500$

$-3.14613600$

$-1.32912300$

$-0.45244700$

0.44337200

$-1.00703500$

$-0.04953200$

0.55638400

$-1.31452500$

$-1.89995100$

$-1.03375100$

$-2.16427300$

$-3.06353000$

$-2.59523900$

$-3.24629800$

$-3.17245400$

$-1.32385200$

$-1.92689500$

$-1.02769700$

$-0.06356300$

0.51911700

$-0.58185800$

$-1.35129100$

0.77708700

1.70011400

1.06273000

$-0.36433400$

0.58110000

1. 26064600

\section{S43}




$\begin{array}{rrr}4.68126200 & -2.32875900 & 2.00134800 \\ 5.19464900 & -0.49852500 & 1.00443400 \\ 4.82494000 & 0.43465600 & 0.03985300 \\ 5.48414800 & 1.27147000 & -0.17279700 \\ 3.62467100 & 0.30596300 & -0.67147000 \\ 2.31910900 & -3.01106700 & 0.86179600 \\ 1.42989600 & -2.96495000 & 0.22626800 \\ 3.30030300 & 1.29119400 & -1.79000500 \\ 2.27021800 & 1.10406300 & -2.10804600 \\ -0.18556100 & 2.14672900 & -0.50402000 \\ 0.04913200 & 2.04969200 & -1.57657200 \\ 1.24523400 & 1.97273800 & 2.26695900 \\ 1.90772100 & 2.53209000 & 1.61370100 \\ 1.45798100 & 0.66661000 & 2.52284600 \\ 0.84798700 & 0.12283900 & 3.23632200 \\ 2.30175400 & 0.14047500 & 2.09592900 \\ -0.41101900 & 3.52411100 & -0.12338500 \\ -0.69591900 & 3.73221500 & 0.90792800 \\ -0.32706300 & 4.55517700 & -0.99800400 \\ -0.04903600 & 4.33587500 & -2.03057800 \\ -0.59081500 & 5.99135800 & -0.67170600 \\ 0.29174200 & 6.61159700 & -0.88274100 \\ -1.40416900 & 6.39036200 & -1.29373200 \\ -0.86458500 & 6.12568000 & 0.37935500 \\ 6.12853000 & -0.37891100 & 1.54731800 \\ 3.08947600 & -4.29655100 & 0.49434500 \\ 3.96855300 & -4.43329200 & 1.13481400 \\ 2.44646800 & -5.17517300 & 0.62222200 \\ 3.43838100 & -4.27781500 & -0.54485700 \\ 1.82159900 & -3.07397100 & 2.31913400 \\ 1.23822200 & -3.98858400 & 2.47747900 \\ 2.65549800 & -3.08518000 & 3.03101800 \\ 1.17720500 & -2.22346700 & 2.55838700 \\ 4.22139000 & 1.04868500 & -3.00525300 \\ 4.15596800 & 0.01462800 & -3.36213100 \\ 3.94821200 & 1.71327400 & -3.83368600 \\ 5.27013800 & 1.24348500 & -2.75091900 \\ 3.38060900 & 2.76133600 & -1.34209900 \\ 3.11173300 & 3.42293700 & -2.17418500 \\ 2.69155500 & 2.96367600 & -0.51723900 \\ 4.39160900 & 3.03648400 & -1.02023400 \\ -1.93800800 & 0.57538000 & 2.93666600 \\ 0.45621900 & 2.52831500 & 2.76181900\end{array}$

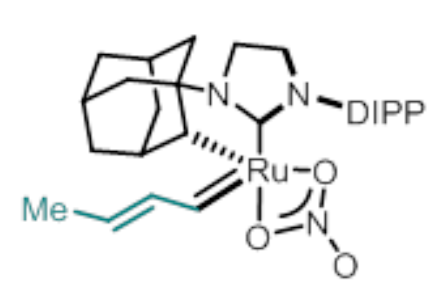

14

SCF Energy (M06, n-octanol): SCF Energy (B3LYP, gas-phase): Enthalpy Correction:

Free-Energy Correction:

$$
\begin{array}{r}
-1613.828636 \\
-1613.455204 \\
0.700146 \\
0.598624
\end{array}
$$

$$
\begin{array}{r}
-0.55034600 \\
0.64128500 \\
-1.11657800 \\
-0.85398500 \\
1.28538700 \\
0.08299400 \\
-0.33585800 \\
-0.66975000
\end{array}
$$

$$
\begin{array}{r}
1.09175700 \\
2.43791000 \\
3.24773800 \\
-1.78724600 \\
-1.37239900 \\
-0.79546100 \\
-3.07837600 \\
-3.90100000
\end{array}
$$


$-1.34330900$

$-0.23286000$

$-1.08983200$

0.68107500

$-0.02253100$

$-1.52622300$

$-1.95732800$

$-2.08219400$

$-1.68753100$

$-2.75446100$

$-1.09052300$

$-1.63630900$

$-1.20303400$

0.40312400

0.82934400

0.54924400

1. 60527200

0.01921100

1.14124200

2.21549200

1.03842800

0.55254900

1.08533500

0.75583800

1.81674500

$-0.94152700$

$-1.37456000$

$-1.05950500$

$-0.61489000$

$-1.98384000$

$-2.38912500$

$-3.43283300$

$-1.48255900$

$-0.14533900$

0.55453200

0.31520200

$-3.03782700$

$-2.52086900$

1.78063100

1.97034200

1. 98125800

2. 28513400

$-3.94884700$

$-4.51670300$

$-4.66943600$

$-3.37455800$

$-3.88204900$

$-4.55788900$

$-4.50486100$

$-3.24646400$

2.08316800

3.12647400

1.92455700

1.43865500

2.73369000

2.65321800

3.77327000

2.52205300

$-1.81944000$

3.05884000

2.83450200

4.33795900

4.54791000
$-3.28841300$

$-2.84772700$

$-3.25098000$

$-3.27849400$

$-1.32951300$

$-1.09296700$

$-0.42414300$

$-2.03980100$

$-0.47034200$

$-0.31389700$

$-1.43334500$

$-2.38764800$

$-1.01177000$

$-1.66821700$

$-2.35171400$

$-2.30679900$

$-2.50721400$

$-3.27143900$

$-0.31147900$

$-0.45428000$

0.12099200

0.65169000

1.60997600

0.00274400

$-0.25810000$

0.87977000

1.57496000

1.34866000

$-0.70941000$

$-0.38282600$

0.20582100

0.47362300

0.45156700

0.10446200

0.28989800

$-0.48143900$

$-0.69268000$

$-1.01787900$

$-0.88478200$

$-1.15456100$

1.33776700

2. 39388700

$-1.85378400$

$-1.58218500$

$-2.10515500$

$-2.75754800$

0.54012600

0.29060400

0.87406200

1. 37711300

$-2.12219200$

$-2.43692600$

$-1.90362400$

$-2.96940300$

0.27170900

0.55521400

$-0.02608900$

1.15657000

0.90987200

0.43480900

$-0.62787100$

0.84987400

1.91723000 


$\begin{array}{rrrr}\mathrm{C} & 0.62370500 & 5.51138200 & -0.03731200 \\ \mathrm{H} & -0.16463400 & 6.27069600 & 0.05930600 \\ \mathrm{H} & 1.56374500 & 6.00519600 & 0.24627100 \\ \mathrm{H} & 0.69177800 & 5.21923800 & -1.09022000 \\ \mathrm{~N} & -0.13866200 & -0.92689900 & 3.46008900 \\ \mathrm{O} & 0.04825600 & -1.47584100 & 4.52455300\end{array}$

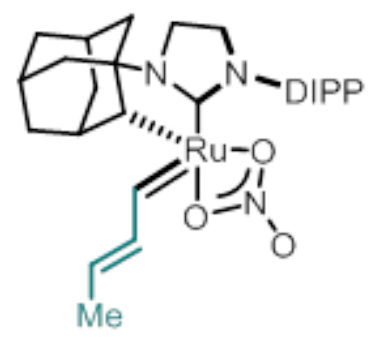

$14 \mathrm{~b}$

SCF Energy (M06, n-octanol):

$-1613.827824$

SCF Energy (B3LYP, gas-phase):

$-1613.459057$

Enthalpy Correction:

0.700296

Free-Energy Correction:

0.598456

\begin{tabular}{|c|c|}
\hline $\mathrm{Ru}$ & -0.58408400 \\
\hline 0 & 0.40601200 \\
\hline 0 & -1.30205800 \\
\hline $\mathrm{N}$ & -0.79588400 \\
\hline $\mathrm{N}$ & 1.34632700 \\
\hline C & 0.12260000 \\
\hline C & -0.23965100 \\
\hline $\mathrm{H}$ & -0.60144800 \\
\hline $\mathrm{H}$ & -0.50044200 \\
\hline $\mathrm{C}$ & 1.26863400 \\
\hline $\mathrm{H}$ & 1.87273100 \\
\hline $\mathrm{H}$ & 1.64161600 \\
\hline C & -2.20100600 \\
\hline C & -2.56857800 \\
\hline $\mathrm{H}$ & -1.84400100 \\
\hline $\mathrm{H}$ & -2.49699800 \\
\hline C & -3.99206400 \\
\hline $\mathrm{H}$ & -4.24080000 \\
\hline C & -4.98765900 \\
\hline $\mathrm{H}$ & -4.96668900 \\
\hline $\mathrm{H}$ & -6.01120500 \\
\hline C & -4.62219900 \\
\hline $\mathrm{H}$ & -5.33204400 \\
\hline C & -3.19550100 \\
\hline $\mathrm{H}$ & -2.92834100 \\
\hline $\mathrm{H}$ & -3.16151200 \\
\hline C & -4.69173100 \\
\hline $\mathrm{H}$ & -4.46963900 \\
\hline $\mathrm{H}$ & -5.71372700 \\
\hline C & -3.68088000 \\
\hline $\mathrm{H}$ & -3.73078200 \\
\hline C & -2.27329100 \\
\hline $\mathrm{H}$ & -2.12889700 \\
\hline C & -4.04449000 \\
\hline $\mathrm{H}$ & -3.35116200 \\
\hline $\mathrm{H}$ & -5.04973400 \\
\hline $\mathrm{C}$ & 2.59149600 \\
\hline $\mathrm{C}$ & 2.92077800 \\
\hline $\mathrm{C}$ & 4.15670000 \\
\hline $\mathrm{H}$ & 4.42780500 \\
\hline C & 5.04549000 \\
\hline C & 4.71578900 \\
\hline 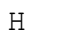 & 5.42239800 \\
\hline $\mathrm{C}$ & 3.49090600 \\
\hline
\end{tabular}

$$
\begin{array}{r}
0.70472700 \\
0.58083200 \\
1.91535100 \\
-1.36245100 \\
-1.20959200 \\
-0.68159700 \\
-2.57642800 \\
-2.72410100 \\
-3.46718600 \\
-2.27363800 \\
-3.13649200 \\
-1.89891400 \\
-1.15267600 \\
-2.01905700 \\
-1.83277700 \\
-3.08754700 \\
-1.67810400 \\
-2.30103200 \\
-1.96125000 \\
-3.02815300 \\
-1.73295700 \\
-1.09906900 \\
-1.29669300 \\
-1.46366700 \\
-0.88092000 \\
-2.52772900 \\
0.39233400 \\
1.02878700 \\
0.63757600 \\
0.68187000 \\
1.74279700 \\
0.34984500 \\
0.89928800 \\
-0.18671700 \\
0.01729600 \\
0.07093200 \\
-0.89757800 \\
-1.57055400 \\
-1.28670500 \\
-1.78343700 \\
-0.38442100 \\
0.24753000 \\
0.93909800 \\
0.00490100
\end{array}
$$

0.54396800

2.52604800

2.33908600

$-1.44363800$

$-0.96692700$

$-0.70191300$

$-2.04641600$

$-3.06773400$

$-1.45609800$

$-1.99229900$

$-1.70198900$

$-2.95404200$

$-1.06297300$

0.17188200

0.97953700

$-0.07804300$

0.65253600

1.52185100

$-0.49162800$

$-0.75606100$

$-0.16537400$

$-1.71778600$

$-2.53177100$

$-2.19899500$

$-3.09039900$

$-2.47913200$

$-1.31874300$

$-2.18595900$

$-0.99567100$

$-0.17797400$

0.09647200

$-0.70656300$

$-1.64319000$

1.04333800

1.87042200

1.40427100

$-0.31642500$

0.88412200

1.47757800

2.40428800

0.90178700

$-0.29292400$

$-0.74255200$

$-0.92677800$ 
2.01972900

1.03886000

3. 19512900

2.13657200

0.03663400

0.55423100

2.58239500

3.56086900

1. 90858900

2.71307200

1.79761700

1.08010700

2.72531400

1. 40665400

4.02448000

3.77319700

5.09787400

3. 84709200

3.43015600

4.49114400

3.10347000

2. 87822400

5.99899600

$-0.13835700$

$-0.62615400$

0.26546400

0.75715500

0.09018400

1. 06048400

$-0.50244900$

$-0.40884900$

$-0.39818700$

$-0.29274600$

$$
\begin{array}{r}
-2.63116700 \\
-2.56919000 \\
0.67512200 \\
0.51184600 \\
2.10143000 \\
1.94697100 \\
-4.04481900 \\
-4.17267200 \\
-4.80859000 \\
-4.24265000 \\
-2.41676100 \\
-3.15608300 \\
-2.54907100 \\
-1.41695900 \\
0.03191300 \\
0.48280100 \\
0.17767500 \\
-1.04714500 \\
2.19718600 \\
2.44116600 \\
2.64981800 \\
2.66979300 \\
-0.17893600 \\
3.48957000 \\
3.70629500 \\
4.51377600 \\
4.28143600 \\
5.96602100 \\
6.48130000 \\
6.47194700 \\
6.11095300 \\
1.42424200 \\
1.70811300
\end{array}
$$

\section{$14 \mathrm{C}$}

SCF Energy (M06, n-octanol): SCF Energy (B3LYP, gas-phase): Enthalpy Correction:

Free-Energy Correction:
1.51296800

1.03162100

$-2.26513900$

$-2.49405100$

$-0.49193400$

$-1.44658400$

1.24816400

1.72638100

1.65481900

0.17762000

3.02220000

3.39892000

3.59149000

3. 22689100

$-3.39732300$

$-4.36502600$

$-3.22749500$

$-3.46805800$

$-2.23169900$

$-2.10555100$

$-3.17593200$

$-1.41367900$

1.38118900

$-0.12320400$

0.82610400

$-0.91060700$

$-1.85688900$

$-0.59139300$

$-0.55835400$

$-1.36652900$

0.37183000

3.10515700

4.27918800

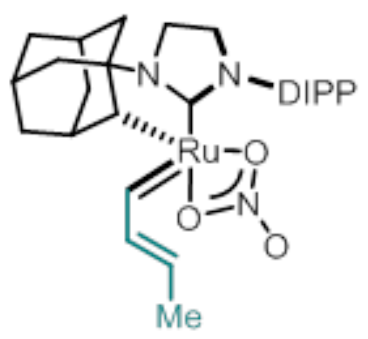

$\mathrm{Ru}$
$\mathrm{O}$
$\mathrm{O}$
$\mathrm{N}$
$\mathrm{N}$
$\mathrm{C}$
$\mathrm{C}$
$\mathrm{H}$
$\mathrm{H}$
$\mathrm{C}$
$\mathrm{H}$
$\mathrm{H}$
$\mathrm{C}$
$\mathrm{C}$
$\mathrm{H}$
$\mathrm{H}$
$\mathrm{C}$

$$
\begin{array}{r}
-0.56347000 \\
0.59129800 \\
-1.25537700 \\
-0.97413800 \\
1.18299100 \\
0.00654000 \\
-0.51884000 \\
-0.91124100 \\
-0.83465200 \\
1.00899100 \\
1.55137300 \\
1.39419300 \\
-2.35104400 \\
-2.77809300 \\
-2.03433500 \\
-2.79762100 \\
-4.16476700
\end{array}
$$

-1613.822199
-1613.452106
0.700186
0.598426

$$
\begin{array}{r}
0.89880700 \\
1.75529200 \\
2.71503700 \\
-1.85255000 \\
-1.64463500 \\
-0.96780100 \\
-3.24404600 \\
-3.83529400 \\
-3.72077000 \\
-3.06571300 \\
-3.71580900 \\
-3.24146800 \\
-1.38165700 \\
-1.53550500 \\
-1.04847300 \\
-2.59840200 \\
-0.90017000
\end{array}
$$
0.07759300
1.83723400
1.20258900
$-0.69860700$
$-0.33570200$
$-0.37568300$
$-0.63764400$
$-1.46964300$
0.30171200
$-0.70703000$
$-0.01593700$
$-1.71943900$
$-0.49800100$
0.98681000
1. 63593200
1. 26859300
1. 20478600 


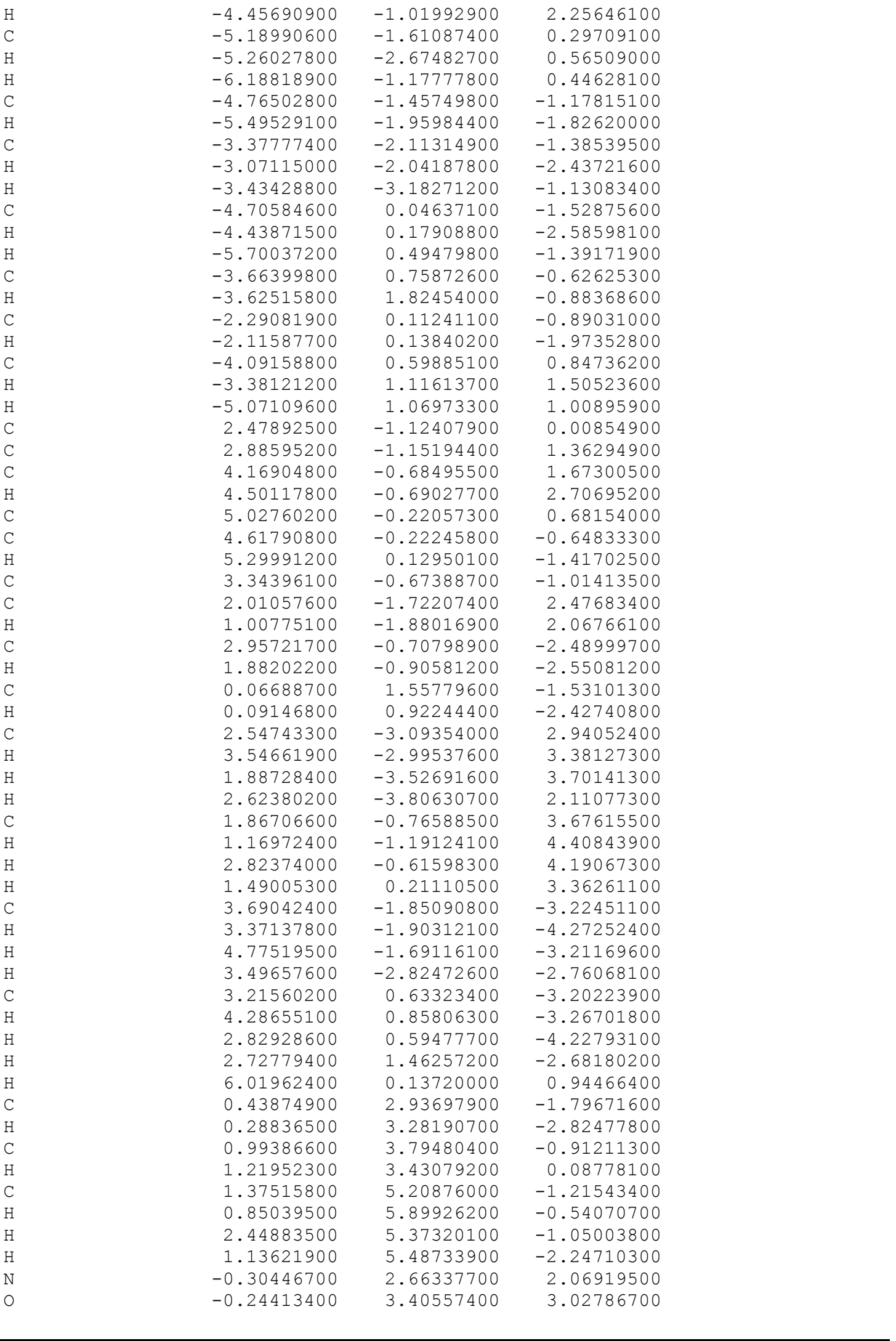




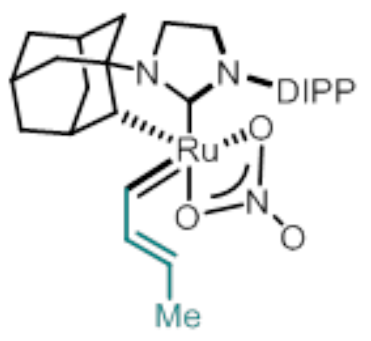

\section{$14 \mathrm{~d}$}

SCF Energy (M06, n-octanol):

$-1613.818996$

SCF Energy (B3LYP, gas-phase):

$-1613.435080$

Enthalpy Correction:

0.700151

Free-Energy Correction:

0.596442

$$
\begin{aligned}
& 0.78565600 \\
& 3.28988100 \\
& \text { 2. } 41915700 \\
& -2.09890600 \\
& -1.61623700 \\
& -1.08809300 \\
& -3.42500100 \\
& -4.10505600 \\
& -3.87375900 \\
& -3.09320500 \\
& -3.52921700 \\
& -3.42878800 \\
& -1.75973700 \\
& -1.82238500 \\
& -1.18571700 \\
& -2.84847500 \\
& -1.34621500 \\
& -1.40361500 \\
& -2.25657700 \\
& -3.29274800 \\
& -1.93507600 \\
& -2.18888300 \\
& -2.83426000 \\
& -2.68821000 \\
& -2.67772700 \\
& -3.72778000 \\
& -0.72898400 \\
& -0.66475200 \\
& -0.38717200 \\
& 0.18601100 \\
& \text { 1.21754200 } \\
& -0.31245700 \\
& -0.35679500 \\
& 0.10972500 \\
& 0.76435500 \\
& 0.47174300 \\
& -0.88468800 \\
& -0.45757600 \\
& 0.23395700 \\
& 0.57921500 \\
& 0.47501300 \\
& 0.02743200 \\
& 0.21464400 \\
& -0.65405700 \\
& -0.74576700 \\
& -1.29875300 \\
& -1.14061000 \\
& -1.48964700 \\
& 1.40066400 \\
& 0.87880000 \\
& -1.64054500 \\
& -1.13825500
\end{aligned}
$$

0.07295700

$-0.76895800$

0.96949000

$-0.30340900$

0.03009100

$-0.11673700$

$-0.10294500$

$-0.92140300$

0.83421700

$-0.05634100$

0.80985100

$-0.95979700$

$-0.26770300$

1.18760700

1.83234100

1.57554900

1.23483600

2.26868900

0.32969600

0.69709000

0.35916600

$-1.11782400$

$-1.76419300$

$-1.15221900$

$-2.17977400$

$-0.79336900$

$-1.61598000$

$-2.65787200$

$-1.60189300$

$-0.71341700$

$-1.07202800$

$-0.81373600$

$-1.87966900$

0.73189900

1. 38883100

0.76517500

0.28645200

1. 60458500

1.83190800

2.83548700

0.79967700

$-0.48795100$

$-1.28596700$

$-0.77360200$

2. 78119000

2.40159900

$-2.19574500$

$-2.24022200$

$-1.51647400$

$-2.46108500$

3. 83144400

4.28509400 


$\begin{array}{lrrr}\mathrm{H} & 2.05969000 & -1.89092000 & 4.63741500 \\ \mathrm{H} & 3.11770900 & -2.57682000 & 3.38787600 \\ \mathrm{C} & 1.53404700 & 0.54605700 & 3.42882100 \\ \mathrm{H} & 0.85159300 & 0.30482100 & 4.25235000 \\ \mathrm{H} & 2.34545600 & 1.15902600 & 3.83850000 \\ \mathrm{H} & 0.98347100 & 1.15980700 & 2.70603200 \\ \mathrm{C} & 4.27835400 & -2.32476500 & -2.56040400 \\ \mathrm{H} & 4.03999100 & -2.70432100 & -3.56121400 \\ \mathrm{H} & 5.33085900 & -2.01729600 & -2.56132600 \\ \mathrm{H} & 4.17972900 & -3.15290700 & -1.84946300 \\ \mathrm{C} & 3.49789300 & -0.01631100 & -3.23925300 \\ \mathrm{H} & 4.53033000 & 0.34470700 & -3.31217700 \\ \mathrm{H} & 3.20847400 & -0.38385600 & -4.23078800 \\ \mathrm{H} & 2.86058900 & 0.83657100 & -2.98925300 \\ \mathrm{H} & 6.02215200 & 1.00816100 & 0.99917300 \\ \mathrm{C} & 0.98175400 & 2.58617000 & -1.66993000 \\ \mathrm{H} & 1.44809700 & 2.73328200 & -2.64815500 \\ \mathrm{C} & 1.17952900 & 3.53983200 & -0.72933000 \\ \mathrm{H} & 0.67328200 & 3.44334800 & 0.23071600 \\ \mathrm{C} & 2.00786300 & 4.76834100 & -0.92628500 \\ \mathrm{H} & 1.39352500 & 5.67158700 & -0.80859000 \\ \mathrm{H} & 2.79852000 & 4.83225800 & -0.16560000 \\ \mathrm{H} & 2.47578800 & 4.79440300 & -1.91580800 \\ \mathrm{~N} & -2.29279200 & 3.32553400 & 0.44365900 \\ \mathrm{O} & -2.76203000 & 4.14758700 & 1.22429000\end{array}$

\section{$14 \mathrm{e}$}

SCF Energy (M06, n-octanol): SCF Energy (B3LYP, gas-phase): Enthalpy Correction:

Free-Energy Correction:

\author{
$-1613.820649$ \\ $-1613.452269$ \\ 0.700874 \\ 0.598247
}

-0.46121700
-1.21226600
-1.29693200
-0.76492100
1.34476700
0.16074800
-0.28153300
-0.54856300
-0.70667700
1.24115300
1.68621300
1.76894600
-2.17056900
-2.75753900
-2.12698700
-2.76375500
-4.19322000
-4.59872900
-5.07207100
-5.12077600
-6.10086200
-4.48596200
-5.11046900
-3.05668700
-2.62962500
$-0.09227500$

2.09981300

0.62928700

$-0.49107100$

0.02278800

$-0.22119700$

$-0.21135100$

$-1.01237800$

0.72722600

$-0.10351200$

0.76497200

$-1.00046700$

$-0.46733600$

0.96132900

1.66475900

1.27424100

0.98852000

2.00387100

$-0.00243400$

0.28972900

0.01647300

$-1.42246700$

$-2.13548900$

$-1.44136700$

$-2.45178100$ 


\begin{tabular}{|c|c|c|c|}
\hline $\mathrm{H}$ & -3.09441500 & -3.35940100 & -1.15105400 \\
\hline $\mathrm{C}$ & -4.45409400 & -0.20945300 & -1.81511100 \\
\hline $\mathrm{H}$ & -4.08143800 & -0.09290300 & -2.84173000 \\
\hline $\mathrm{H}$ & -5.47552700 & 0.19661100 & -1.79812900 \\
\hline C & -3.55072600 & 0.57985800 & -0.82576200 \\
\hline $\mathrm{H}$ & -3.52602900 & 1.63695600 & -1.11598600 \\
\hline $\mathrm{C}$ & -2.13710400 & -0.02075300 & -0.94682600 \\
\hline $\mathrm{H}$ & -1.89670400 & -0.05617300 & -2.01696800 \\
\hline C & -4.14885400 & 0.43799500 & 0.58932600 \\
\hline $\mathrm{H}$ & -3.56049000 & 0.99418100 & 1.32389700 \\
\hline $\mathrm{H}$ & -5.16325000 & 0.86013000 & 0.60199300 \\
\hline C & 2.59669700 & -0.94478600 & 0.32162200 \\
\hline C & 2.88337800 & -0.58459800 & 1.65911200 \\
\hline $\mathrm{C}$ & 4.13087600 & -0.00730100 & 1.93042000 \\
\hline $\mathrm{H}$ & 4.37344000 & 0.27946300 & 2.94944900 \\
\hline C & 5.06909300 & 0.19453700 & 0.92306600 \\
\hline $\mathrm{C}$ & 4.77525800 & -0.18081800 & -0.38467000 \\
\hline $\mathrm{H}$ & 5.51657200 & -0.02589500 & -1.16342800 \\
\hline $\mathrm{C}$ & 3.54136500 & -0.75433300 & -0.71350100 \\
\hline $\mathrm{C}$ & 1.91806500 & -0.85195300 & 2.81098600 \\
\hline $\mathrm{H}$ & 0.95461900 & -1.14570400 & 2.38495700 \\
\hline C & 3.27294200 & -1.17862800 & -2.15500700 \\
\hline H & 2.21441100 & -1.44703700 & -2.23383600 \\
\hline $\mathrm{C}$ & 0.17533700 & 1.33707700 & -1.81493800 \\
\hline $\mathrm{H}$ & 0.06214500 & 0.63398300 & -2.65622200 \\
\hline $\mathrm{C}$ & 2.42159700 & -2.02425900 & 3.68003600 \\
\hline 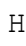 & 3.37928500 & -1.78135700 & 4.15589700 \\
\hline $\mathrm{H}$ & 1.69963100 & -2.24865700 & 4.47397900 \\
\hline $\mathrm{H}$ & 2.56999400 & -2.93482900 & 3.08748800 \\
\hline $\mathrm{C}$ & 1.66450700 & 0.39517700 & 3.67767700 \\
\hline $\mathrm{H}$ & 0.92376900 & 0.16769500 & 4.45206200 \\
\hline $\mathrm{H}$ & 2.57665300 & 0.73637800 & 4.18111800 \\
\hline $\mathrm{H}$ & 1.26784900 & 1.22029300 & 3.07978800 \\
\hline $\mathrm{C}$ & 4.11068700 & -2.41853700 & -2.53374500 \\
\hline $\mathrm{H}$ & 3.86863000 & -2.75203500 & -3.55004400 \\
\hline $\mathrm{H}$ & 5.18278400 & -2.19049500 & -2.50305200 \\
\hline $\mathrm{H}$ & 3.93468400 & -3.25602400 & -1.84932600 \\
\hline c & 3.52586000 & -0.03941800 & -3.16033600 \\
\hline $\mathrm{H}$ & 4.58739400 & 0.22925600 & -3.21074500 \\
\hline $\mathrm{H}$ & 3.21937100 & -0.34942400 & -4.16653300 \\
\hline $\mathrm{H}$ & 2.96361000 & 0.85882700 & -2.89019500 \\
\hline $\mathrm{H}$ & 6.03225400 & 0.64011500 & 1.15792700 \\
\hline c & 0.83622000 & 2.56383300 & -2.23524100 \\
\hline $\mathrm{H}$ & 1.21732800 & 2.59396800 & -3.26039300 \\
\hline C & 1.00285500 & 3.67762200 & -1.47997900 \\
\hline $\mathrm{H}$ & 0.59149200 & 3.69693700 & -0.47198700 \\
\hline C & 1.68405500 & 4.92356600 & -1.94661300 \\
\hline $\mathrm{H}$ & 0.99803400 & 5.78089000 & -1.90344600 \\
\hline $\mathrm{H}$ & 2.52847300 & 5.17467900 & -1.28928700 \\
\hline 4 & 2.05888700 & 4.82943100 & -2.97110800 \\
\hline $\mathrm{N}$ & -1.48487900 & 2.50333600 & 1.85383800 \\
\hline$\Omega$ & -1.89797400 & 3.26905100 & 2.70224500 \\
\hline
\end{tabular}

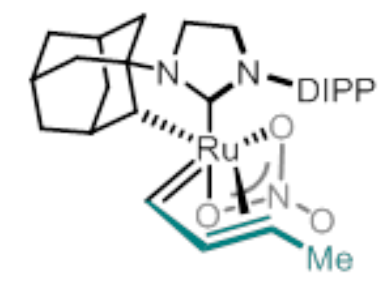

\section{4-constained}

SCF Energy (M06, n-octanol): $\quad-1613.807519$

SCF Energy (B3LYP, gas-phase): $\quad-1613.430740$ 


\begin{tabular}{|c|c|c|c|}
\hline $\mathrm{Ru}$ & -0.47726200 & 0.81070200 & -0.66921700 \\
\hline 0 & -1.09310500 & 2.12171800 & 1.16831100 \\
\hline 0 & -1.47699800 & 2.81376200 & -0.85328400 \\
\hline $\mathrm{N}$ & -0.81436800 & -1.88616900 & 0.37741100 \\
\hline $\mathrm{N}$ & 1.31581700 & -1.40734700 & 0.60936700 \\
\hline $\mathrm{C}$ & 0.12854500 & -0.95004700 & 0.13502900 \\
\hline $\mathrm{C}$ & -0.32889300 & -2.97489300 & 1.23309700 \\
\hline $\mathrm{H}$ & -0.63022200 & -3.95335400 & 0.84815500 \\
\hline $\mathrm{H}$ & -0.72546700 & -2.87088200 & 2.25148300 \\
\hline $\mathrm{C}$ & 1.19752100 & -2.76381900 & 1.18456900 \\
\hline $\mathrm{H}$ & 1.66992900 & -2.80830900 & 2.17032700 \\
\hline $\mathrm{H}$ & 1.69232700 & -3.49536000 & 0.53489200 \\
\hline $\mathrm{C}$ & -2.21951700 & -1.47683500 & 0.23724400 \\
\hline $\mathrm{C}$ & -2.75564200 & -0.91335400 & 1.57827500 \\
\hline $\mathrm{H}$ & -2.10204900 & -0.10445900 & 1.92237400 \\
\hline $\mathrm{H}$ & -2.74978100 & -1.70165300 & 2.34554500 \\
\hline $\mathrm{C}$ & -4.19038100 & -0.38380000 & 1.38576000 \\
\hline $\mathrm{H}$ & -4.56038300 & 0.00694700 & 2.34274600 \\
\hline $\mathrm{C}$ & -5.10084900 & -1.53069200 & 0.90471600 \\
\hline $\mathrm{H}$ & -5.13686400 & -2.33085600 & 1.65816300 \\
\hline $\mathrm{H}$ & -6.12918200 & -1.16748900 & 0.77380400 \\
\hline $\mathrm{C}$ & -4.56361300 & -2.08090500 & -0.43233800 \\
\hline $\mathrm{H}$ & -5.21045000 & -2.89569700 & -0.78429100 \\
\hline $\mathrm{C}$ & -3.13429500 & -2.63567800 & -0.21658100 \\
\hline $\mathrm{H}$ & -2.74319200 & -3.07065800 & -1.14590700 \\
\hline $\mathrm{H}$ & -3.16156600 & -3.43859200 & 0.53614200 \\
\hline $\mathrm{C}$ & -4.55008900 & -0.94102300 & -1.47629300 \\
\hline $\mathrm{H}$ & -4.21381600 & -1.32168000 & -2.45025000 \\
\hline $\mathrm{H}$ & -5.57261800 & -0.56222500 & -1.61695800 \\
\hline $\mathrm{C}$ & -3.61445400 & 0.20412600 & -0.99850000 \\
\hline $\mathrm{H}$ & -3.60477500 & 1.00601100 & -1.74615000 \\
\hline $\mathrm{C}$ & -2.20034700 & -0.39782100 & -0.86710000 \\
\hline $\mathrm{H}$ & -2.00330400 & -0.93477100 & -1.80734900 \\
\hline $\mathrm{C}$ & -4.16271500 & 0.74620500 & 0.33779700 \\
\hline $\mathrm{H}$ & -3.55111300 & 1.57284200 & 0.70799400 \\
\hline $\mathrm{H}$ & -5.17680100 & 1.14183300 & 0.18654600 \\
\hline $\mathrm{C}$ & 2.58626600 & -0.74234600 & 0.52325800 \\
\hline $\mathrm{C}$ & 2.97252500 & 0.12637700 & 1.57043200 \\
\hline $\mathrm{C}$ & 4.22316600 & 0.75157700 & 1.47664200 \\
\hline $\mathrm{H}$ & 4.54064300 & 1.42675900 & 2.26607900 \\
\hline $\mathrm{C}$ & 5.07037200 & 0.51275300 & 0.39880800 \\
\hline $\mathrm{C}$ & 4.68377300 & -0.36776500 & -0.60834800 \\
\hline $\mathrm{H}$ & 5.35824400 & -0.55779400 & -1.43818400 \\
\hline $\mathrm{C}$ & 3.44199000 & -1.01340800 & -0.56961100 \\
\hline $\mathrm{C}$ & 2.11130200 & 0.35788600 & 2.80959700 \\
\hline $\mathrm{H}$ & 1.15075000 & -0.14070400 & 2.65036400 \\
\hline $\mathrm{C}$ & 3.07572000 & -2.00974300 & -1.66686800 \\
\hline $\mathrm{H}$ & 2.01038200 & -2.24159000 & -1.56619300 \\
\hline $\mathrm{C}$ & 0.09552200 & 0.40147000 & -2.38944100 \\
\hline $\mathrm{H}$ & -0.15610500 & -0.38278600 & -3.11358100 \\
\hline $\mathrm{C}$ & 2.76918600 & -0.28151600 & 4.05111400 \\
\hline $\mathrm{H}$ & 3.72436000 & 0.20196300 & 4.28803600 \\
\hline $\mathrm{H}$ & 2.11602400 & -0.17674900 & 4.92528000 \\
\hline $\mathrm{H}$ & 2.96884000 & -1.34912300 & 3.90038600 \\
\hline $\mathrm{C}$ & 1.81058400 & 1.84777900 & 3.05941700 \\
\hline $\mathrm{H}$ & 1.19409100 & 1.95740800 & 3.95887500 \\
\hline $\mathrm{H}$ & 2.72793600 & 2.42792700 & 3.21539700 \\
\hline $\mathrm{H}$ & 1.25267000 & 2.28895100 & 2.22870800 \\
\hline $\mathrm{C}$ & 3.86946700 & -3.32365100 & -1.49996200 \\
\hline $\mathrm{H}$ & 3.56056200 & -4.05867700 & -2.25282300 \\
\hline $\mathrm{H}$ & 4.94469800 & -3.14815500 & -1.62417500 \\
\hline $\mathrm{H}$ & 3.72328400 & -3.76855100 & -0.50931000 \\
\hline $\mathrm{C}$ & 3.28415 & -1.44303700 & -3.08391700 \\
\hline
\end{tabular}

\section{S52}




$\begin{array}{rrrr}\mathrm{H} & 4.34561800 & -1.28058800 & -3.30452400 \\ \mathrm{H} & 2.90182900 & -2.14949100 & -3.83012400 \\ \mathrm{H} & 2.76258900 & -0.49025300 & -3.21230700 \\ \mathrm{H} & 6.03780000 & 1.00561400 & 0.34785800 \\ \mathrm{C} & 0.92139600 & 1.53705000 & -2.73123000 \\ \mathrm{H} & 0.96151800 & 1.98174000 & -3.72853700 \\ \mathrm{C} & 1.45648000 & 2.16793500 & -1.65519900 \\ \mathrm{H} & 1.53518200 & 1.63301100 & -0.69572900 \\ \mathrm{C} & 2.10204400 & 3.51956200 & -1.66836300 \\ \mathrm{H} & 1.54300100 & 4.20948700 & -1.02121200 \\ \mathrm{H} & 3.12817100 & 3.48066900 & -1.27974000 \\ \mathrm{H} & 2.12202500 & 3.94631600 & -2.67632600 \\ \mathrm{~N} & -1.52604300 & 3.06392200 & 0.40863400 \\ \mathrm{O} & -1.96094400 & 4.11388600 & 0.84624000\end{array}$

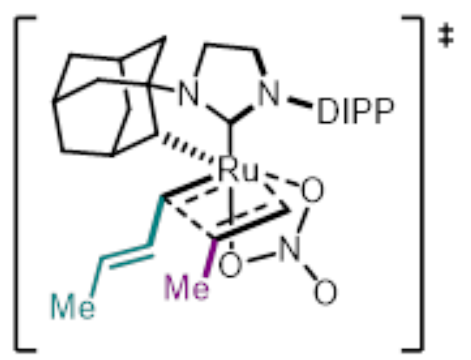

\section{5-TS}

SCF Energy (M06, n-octanol): $\quad-1731.672626$

SCF Energy (B3LYP, gas-phase): $\quad-1731.354204$

Enthalpy Correction:

0.787843

Free-Energy Correction:

0.681266

Ru
O
O
O
C
C
H
H
C
H
H
C
C
H
H
C
H
C
H
H
C
H
C
H
H
C
H
H
C
H
C
C

$$
\begin{array}{r}
0.74447600 \\
0.04675600 \\
2.10788300 \\
-1.39979000 \\
-1.13789400 \\
-0.69830900 \\
-2.48663500 \\
-2.51754800 \\
-3.45942600 \\
-2.10556500 \\
-2.95544600 \\
-1.62241600 \\
-1.32738600 \\
-2.46147600 \\
-2.39414100 \\
-3.44179100 \\
-2.33264200 \\
-3.15133400 \\
-2.41046900 \\
-3.38170300 \\
-2.32998300 \\
-1.26525800 \\
-1.30831500 \\
-1.43579300 \\
-0.65988300 \\
-2.41019200 \\
0.09099000 \\
0.91686500 \\
0.20378900 \\
0.16586800 \\
1.13335400 \\
0.04647100 \\
0.80046800 \\
-0.97847400
\end{array}
$$

0.52407200

2.47034200

1.77933700

$-1.42863700$

$-0.91863400$

$-0.62976200$

$-2.16831700$

$-3.20825300$

$-1.70811600$

$-2.04007400$

$-1.81404200$

$-2.95126900$

$-1.19758100$

$-0.24167300$

0.69292400

$-0.69307500$

0.04825900

0.71215200

$-1.27327400$

$-1.76356700$

$-1.07142900$

$-2.20368300$

$-3.14416000$

$-2.51725000$

$-3.21518200$

$-3.00288500$

$-1.50821000$

$-2.17498900$

$-1.29851000$

$-0.18468500$

0.29651400

$-0.56538200$

$-1.35084100$

0.73423600

\section{S53}




\begin{tabular}{|c|c|c|}
\hline-3.71623200 & -0.93876800 & 1.69551700 \\
\hline-5.30758700 & -0.86208100 & 0.93151200 \\
\hline 2.77043600 & -0.96028700 & -0.17181900 \\
\hline 3.00245100 & -1.77727400 & 0.96487200 \\
\hline 4.21325700 & -1.62394300 & 1.65145100 \\
\hline 4.40699100 & -2.23137800 & 2.53056800 \\
\hline 5.18043600 & -0.72046800 & 1.22016400 \\
\hline 4.96156200 & 0.03145800 & 0.07058600 \\
\hline 5.73593500 & 0.70824600 & -0.27855300 \\
\hline 3.76638300 & -0.07819000 & -0.65309400 \\
\hline 2.02616000 & -2.86826800 & 1.40969400 \\
\hline 1.07840600 & -2.70863600 & 0.88852100 \\
\hline 3.61702500 & 0.68675500 & -1.96683500 \\
\hline 2.56711800 & 0.62296400 & -2.27122100 \\
\hline 0.88996000 & 0.79252200 & 2.08609000 \\
\hline 1.51501000 & -0.09026400 & 2.06020500 \\
\hline 0.33498200 & 2.11876800 & -0.45514800 \\
\hline 1.16794500 & 1.92987500 & -1.13745800 \\
\hline 1.34991300 & 1.96082600 & 1.38754900 \\
\hline 2.27691500 & 1.82083900 & 0.83621200 \\
\hline 1.18441800 & 3.32002200 & 2.03797600 \\
\hline 1.91369300 & 3.41039200 & 2.85445100 \\
\hline 1.35986500 & 4.14144900 & 1.33757000 \\
\hline 0.18265000 & 3.42957300 & 2.46206900 \\
\hline 0.36682700 & 0.94914800 & 3.02704000 \\
\hline-0.25073900 & 3.44387800 & -0.60813200 \\
\hline-1.06394600 & 3.71357600 & 0.06442500 \\
\hline 0.11367300 & 4.30617100 & -1.57995600 \\
\hline 0.93029300 & 4.02725300 & -2.24886600 \\
\hline-0.53612700 & 5.63181900 & -1.84148500 \\
\hline-0.97501300 & 5.66769100 & -2.84847100 \\
\hline-1.33004400 & 5.84051200 & -1.11718200 \\
\hline 0.19750500 & 6.44868500 & -1.79344600 \\
\hline 4.47054900 & 0.02811800 & -3.07318600 \\
\hline 4.31424600 & 0.53469900 & -4.03313000 \\
\hline 5.53814600 & 0.08925600 & -2.83082600 \\
\hline 4.22309600 & -1.03097400 & -3.20278400 \\
\hline 3.97751400 & 2.17997500 & -1.85078400 \\
\hline 5.03865800 & 2.32634400 & -1.61941600 \\
\hline 3.78076300 & 2.68737600 & -2.80263600 \\
\hline 3.39322600 & 2.68335300 & -1.07419500 \\
\hline 2.56116200 & -4.25852300 & 0.99932300 \\
\hline 2.76278100 & -4.32066800 & -0.07603600 \\
\hline 3.49729100 & -4.48846000 & 1.52200300 \\
\hline 1.83417000 & -5.03868100 & 1.25406800 \\
\hline 1.71660600 & -2.85333200 & 2.91882300 \\
\hline 0.98355900 & -3.63399400 & 3.15164400 \\
\hline 2.60866300 & -3.05384600 & 3.52355100 \\
\hline 1.29459200 & -1.89720900 & 3.24016600 \\
\hline 6.11269500 & -0.61751700 & 1.76910200 \\
\hline-2.27817500 & 1.24289900 & 2.62607400 \\
\hline-3.05470900 & 1.57004100 & 3.50999400 \\
\hline
\end{tabular}




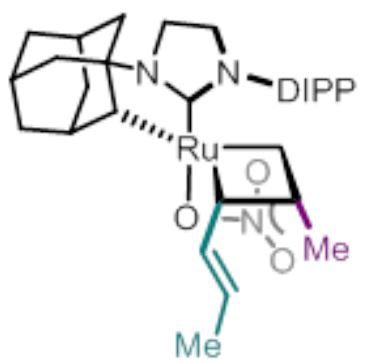

\section{$16 a$}

SCF Energy (M06, n-octanol):

$-1731.686644$

SCF Energy (B3LYP, gas-phase):

$-1731.363260$

Enthalpy Correction:

0.789495

Free-Energy Correction:

0.682258

-0.61400100
-2.12859600
-1.81078900
-0.52335700
1.58074300
0.30584700
0.14474600
-0.17346400
-0.07276300
1.61565600
2.27076500
1.99669900
-1.96567800

-1.96567800
-2.39494300

$-1.81382300$

$-2.17008900$

$-3.90246500$

$-4.18965400$

$-4.70408500$

$-4.53178300$

$-5.77958300$

$-4.28367200$

$-4.85803600$

$-2.78069400$

$-2.45986500$

$-2.59624200$

$-4.54675900$

$-4.27803600$

$-5.61756600$

$-3.72951700$

$-3.91953200$

$-2.24860900$

$-2.01926500$

$-4.17533800$

$-3.64509700$

$-5.24825900$

2.77946300

2. 96904800

4.16562400

4.32845500

5.15799600

4.97830800

5.77001100

3.79837200

1. 96568600

1.01931500

3. 68750100

2. 64379200

0.72121000

1. 43528800

0.58425700

1. 27480500

$$
\begin{aligned}
& 0.74768600 \\
& 0.36146100 \\
& 2.24634900
\end{aligned}
$$

$-1.44486000$

$-1.15195100$

$-0.71842400$

$-2.53797200$

$-2.59185100$

$-3.50477800$

$-2.13661100$

$-2.97475700$

$-1.66245200$

$-1.40660800$

$-2.56281400$

$-2.49919100$

$-3.53099000$

$-2.46278200$

$-3.29725700$

$-2.53218600$

$-3.49286200$

$-2.47250700$

$-1.36604200$

$-1.40433800$

$-1.49874200$

$-0.70130200$

$-2.45834700$

$-0.02426800$

0.81561000

0.07584100

0.04120700

0.99948000

$-0.06039600$

0.75552000

$-1.12520200$

$-1.07706100$

$-1.02800700$

$-0.95135400$

$-1.71404800$

$-1.54522600$

$-2.11065300$

$-0.67977000$

0.01947500

0.66933000

$-0.10569800$

$-2.76832700$

$-2.59277000$

0.61243600

0.55091300

0.85298400

0.04839700

2.16870100

1.85302200
0.47260900

2. 61940100

1.56409600

$-1.41513100$

$-0.87423200$

$-0.61622400$

$-2.12542700$

$-3.17011600$

$-1.65066200$

$-1.98087200$

$-1.73270400$

$-2.89368600$

$-1.17039100$

$-0.22719700$

0.70129700

$-0.69646100$

0.08049100

0.73356200

$-1.23469000$

$-1.74132500$

$-1.02107500$

$-2.15305000$

$-3.08803700$

$-2.48379900$

$-3.16734400$

$-2.98904700$

$-1.43721400$

$-2.09240500$

$-1.21254700$

$-0.11481500$

0.37805500

$-0.50073300$

$-1.23005000$

0.79185600

1.74579600

1.00756500

$-0.10316700$

1.07806500

1.78552100

2.69801400

1.33380600

0.14501400

$-0.21595000$

$-0.60075100$

1.54959000

1.03128500

$-1.94444900$

$-2.27036500$

1. 98619600

2.13168500

$-0.30941400$

$-1.09166600$ 


$\begin{array}{rrrr}\mathrm{C} & 1.28795500 & 2.04148600 & 1.14258800 \\ \mathrm{H} & 2.31508000 & 1.76154900 & 0.89323300 \\ 1.26336100 & 3.35218200 & 1.94042700 \\ \mathrm{C} & 1.88899100 & 3.25142600 & 2.83459500 \\ \mathrm{H} & 1.64698600 & 4.18741400 & 1.34395200 \\ \mathrm{H} & 0.24686400 & 3.59330700 & 2.26275000 \\ \mathrm{H} & 0.25649100 & 1.16381100 & 2.92266300 \\ \mathrm{C} & -0.09270200 & 3.43342800 & -0.65687900 \\ \mathrm{H} & -0.76005200 & 3.86274400 & 0.08763200 \\ \mathrm{H} & 0.03288800 & 4.04359500 & -1.84817600 \\ \mathrm{C} & 0.70128900 & 3.60920900 & -2.59512500 \\ \mathrm{H} & -0.68270500 & 5.30081800 & -2.24945900 \\ \mathrm{H} & -1.31307600 & 5.13783800 & -3.13506000 \\ \mathrm{H} & -1.32380900 & 5.67093300 & -1.44244400 \\ \mathrm{C} & 0.02609500 & 6.09796800 & -2.51466100 \\ \mathrm{H} & 4.55813100 & -0.09308400 & -3.00765500 \\ \mathrm{H} & 4.42875000 & 0.38161900 & -3.98766600 \\ \mathrm{H} & 5.62064200 & -0.03338200 & -2.74375100 \\ \mathrm{C} & 4.30365200 & -1.15393000 & -3.10658500 \\ \mathrm{H} & 4.06129400 & 2.10532800 & -1.87076700 \\ \mathrm{H} & 5.12026400 & 2.24786200 & -1.62725600 \\ \mathrm{H} & 3.88481800 & 2.58336400 & -2.84143300 \\ \mathrm{C} & 3.46911200 & 2.63818500 & -1.12089700 \\ \mathrm{H} & 2.45997600 & -4.18071400 & 1.16413500 \\ \mathrm{H} & 2.64620600 & -4.27278700 & 0.08815600 \\ \mathrm{H} & 3.39687900 & -4.42135200 & 1.68050800 \\ \mathrm{C} & 1.71733700 & -4.93671400 & 1.44556900 \\ \mathrm{H} & 1.66540300 & -2.71619000 & 3.05946200 \\ \mathrm{H} & 0.89505200 & -3.45548100 & 3.30662300 \\ \mathrm{H} & 2.54847700 & -2.95433900 & 3.66366700 \\ \mathrm{H} & -2.37663300 & 1.59563700 & 2.54149700 \\ \mathrm{~N} & -3.10790800 & 2.18848700 & 3.32429600\end{array}$

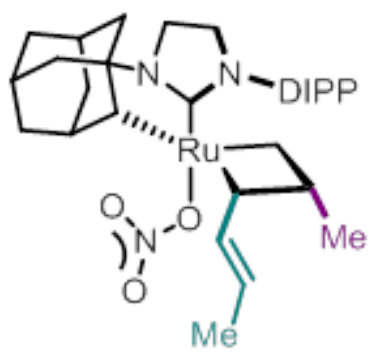

$16 b$

SCF Energy (M06, n-octanol): $-1731.691230$ SCF Energy (B3LYP, gas-phase): Enthalpy Correction:

Free-Energy Correction:

$-1731.359576$

0.789790

0.682336

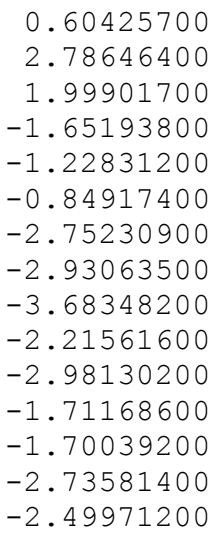

$$
\begin{array}{r}
-0.50757300 \\
0.16708700 \\
-1.66956700 \\
1.30453800 \\
0.81895500 \\
0.55099400 \\
1.95147700 \\
2.96695700 \\
1.37586700 \\
1.92336300 \\
1.73048200 \\
2.86450500 \\
0.95996300 \\
-0.17291600 \\
-1.01749900
\end{array}
$$


1. 92098000

3.65438000

3.80841200

4.55841900

4. 34600600

5.61328300

4.32194000

4.96592800

2.84436400

2.66379700

2. 61267600

4.65389300

4.52388800

5.70723700

3.73800300

3.97554200

2. 29007600

2. 17786700

3.97339900

3. 34435300

5.01731800

$-2.78902200$

$-3.08536800$

$-4.27833800$

$-4.51979400$

$-5.17240100$

$-4.89858100$

$-5.61779000$

$-3.71522800$

$-2.20758700$

$-1.18510400$

$-3.51088500$

$-2.45477500$

$-0.35021100$

$-1.00530200$

$-0.67281800$

$-1.41299100$

$-1.11711500$

$-2.15202700$

$-1.03558800$

$-1.48677400$

$-1.57591500$

0.00418200

0.23937100

$-0.18450900$

0.40467000

$-0.40996600$

$-0.99608200$

0.11611900

0.77301300

0.69283900

$-0.69816400$

$-4.35636500$

$-4.16605600$

$-5.42613100$

$-4.13941400$

$-3.84145400$

$-4.91237400$

$-3.57079600$

$-3.29936600$

$-2.68738800$

$-2.67930600$

$-3.71217600$
$-3.74384700$

$-2.69828300$

$-3.44107100$

$-3.03080700$

$-4.04656300$

$-3.01484500$

$-1.99941700$

$-2.23358300$

$-2.05822700$

$-1.35037600$

$-3.06538400$

$-0.59064900$

0.16223900

$-0.54986800$

$-0.25028900$

0.75385600

$-0.29075200$

0.42813200

$-1.28631000$

$-1.05355500$

$-1.23766900$

$-0.88685600$

$-1.55115300$

$-1.22152600$

$-1.70117000$

$-0.30152500$

0.29017700

0.98105000

0.00754500

$-2.67046600$

$-2.51523900$

0.61037100

0.49077800

0.49954700

$-0.34312100$

2.03347100

1.67236200

1.73300000

1.39523200

2.95710900

2.71572700

3.81006900

3.24639400

0.79309400

3.37260000

3. 91867700

3.94438800

3.39990600

5.28329600

5.18725500

5.75748500

5.96169900

$-0.15149100$

0.23976700

$-0.03391600$

$-1.22468800$

2.11391500

2.30056100

2.50537900

2.69284600

$-4.04399200$

$-4.09943300$

$-4.24610900$
0.17948800

$-0.62211900$

$-1.41615000$

0.58274200

0.94616000

0.27788500

1.70609200

2.56388500

2.16130800

2. 98100800

2.53901600

1.17157500

1.95960600

0.86087200

$-0.03528200$

$-0.38636000$

0.46923200

1. 30579100

$-1.15201000$

$-2.02127400$

$-1.49024600$

0.11681300

$-1.10310500$

$-1.75826300$

$-2.70121300$

$-1.21812400$

0.00968800

0.43874800

0.70550300

$-1.66919800$

$-1.31303200$

2.09463500

2. 36069700

$-2.18577500$

$-2.38368200$

$-0.04052500$

0.67063900

$-1.54003200$

$-1.43645500$

$-2.46254800$

$-3.43144100$

$-2.03683200$

$-2.63502800$

$-3.05880600$

0.31750600

$-0.41270700$

1. 51410800

2. 25683200

1. 93539700

2. 81107500

1.13527400

2. 22674200

3. 14039100

4.14694500

2. 93105100

3. 14508200

2.16761400

2.03065400

3.15509400

1. 41436500

$-1.14656700$

$-0.05365200$

$-1.47987100$ 


$\begin{array}{lrrr}\mathrm{H} & -2.04374200 & -4.84495200 & -1.52939300 \\ \mathrm{C} & -2.15709300 & -2.71583100 & -3.20881400 \\ \mathrm{H} & -1.39521800 & -3.43501600 & -3.52964900 \\ \mathrm{H} & -3.10995400 & -3.04472900 & -3.63941500 \\ \mathrm{H} & -1.90802500 & -1.74490900 & -3.64716500 \\ \mathrm{H} & -6.09152400 & -0.06155200 & -1.74596100 \\ \mathrm{~N} & 2.65061800 & 2.89296700 & -1.06135000 \\ \mathrm{O} & 3.12419800 & 3.79980900 & -1.74619800\end{array}$

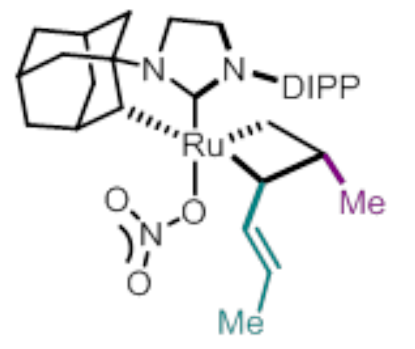

$16 \mathrm{c}$

SCF Energy (M06, n-octanol): SCF Energy (B3LYP, gas-phase): Enthalpy Correction:

Free-Energy Correction:

$$
\begin{array}{r}
-1731.687893 \\
-1731.362112 \\
0.789682 \\
0.682226
\end{array}
$$

$\mathrm{O}$

$\mathrm{N}$

$\mathrm{N}$

C

C

$\mathrm{H}$

$\mathrm{H}$

C

$\mathrm{H}$

C

H

C

$\mathrm{H}$

$\mathrm{H}$

C

$\mathrm{H}$

C

$\mathrm{H}$

$\mathrm{H}$

C

\begin{abstract}
0.68770100
1.76443700

1.82160400

0.77716100

$-1.37144600$

$-0.13850200$

0.22760200

0.63421900

0.44917900

$-1.26964300$

$-1.89739300$

$-1.60402800$

2. 19861900

2. 76591000

2.15283300

2. 70264400

4.23087600

4. 62016000

5.06922000

5.05399600

6.11821900

4. 50609100

5.09981000

3. 04272600

2. 63151900

3. 00400200

4.56439300

4.19142800

5.60692100

3. 71925000

3.77961300

2. 26683300

1. 92789800

4.28622800

3.71655500

5. 32493300

$-2.63433000$

$-3.61055600$

0.64202400

2.58033500

2. 38387100

$-2.11279300$

$-1.75780500$

$-1.15291500$

$-3.46771000$

$-4.04818000$

$-4.00451400$

$-3.17602400$

$-3.81387300$

$-3.28257700$

$-1.77745600$

$-2.08152100$

$-1.57187300$

$-3.16117100$

$-1.60848200$

$-1.82961600$

$-2.35461700$

$-3.43641200$

$-2.03445500$

$-2.05873400$

$-2.58991900$

$-2.54784300$

$-2.37603600$

$-3.63065100$

$-0.54029800$

$-0.31114800$

$-0.19528300$

0.21557200

1.28484500

$-0.26772500$

$-0.10007100$

$-0.09018900$

0.45534800

0.26160100

$-1.10324500$

$-1.13116600$
\end{abstract}

$-0.50640600$

0.99187300

$-1.18140500$

0.33470200

0.10483400

0.05533300

0.37218000

1. 20483400

$-0.56106600$

0.52504300

$-0.10174900$

1.56514300

0.24186200

$-1.17083900$

$-1.92435400$

$-1.37058800$

$-1.25922500$

$-2.26181200$

$-0.20096100$

$-0.39713800$

$-0.25794100$

1.20495600

1.96064800

1.28514500

2. 28878500

1.09321800

1.46567700

2.47285000

1.42414700

0.40419000

0.61350700

0.54605800

1.57913400

$-0.99728400$

$-1.75860400$

$-1.06244100$

0.32517500

$-0.69800600$

S58 


\begin{tabular}{|c|c|c|c|}
\hline $\mathrm{C}$ & -4.83453900 & -0.49281200 & -0.45878700 \\
\hline $\mathrm{H}$ & -5.59644300 & -0.49167600 & -1.23285400 \\
\hline C & -5.09663800 & 0.13140800 & 0.75743100 \\
\hline $\mathrm{C}$ & -4.14678600 & 0.09667200 & 1.77469800 \\
\hline $\mathrm{H}$ & -4.37424800 & 0.55499500 & 2.73318900 \\
\hline $\mathrm{C}$ & -2.90769200 & -0.53073500 & 1.59178000 \\
\hline C & -3.40195000 & -1.90240100 & -2.00024200 \\
\hline $\mathrm{H}$ & -2.33742400 & -2.14900500 & -2.06973100 \\
\hline $\mathrm{C}$ & -1.94031000 & -0.62231900 & 2.77350000 \\
\hline $\mathrm{H}$ & -1.02555700 & -1.11532900 & 2.43438300 \\
\hline C & 0.02624400 & 0.38802300 & -2.36585700 \\
\hline $\mathrm{C}$ & -1.02827600 & 1.80688900 & -0.36976100 \\
\hline C & -1.05414000 & 1.46678200 & -1.91979700 \\
\hline $\mathrm{H}$ & -0.42750100 & -0.54830600 & -2.69817700 \\
\hline $\mathrm{H}$ & 0.71615900 & 0.79256000 & -3.11374900 \\
\hline $\mathrm{H}$ & -2.01760200 & 0.96327100 & -2.03207600 \\
\hline $\mathrm{H}$ & -1.83380000 & 1.29793200 & 0.15228600 \\
\hline $\mathrm{C}$ & -0.93765700 & 3.21560800 & 0.03250800 \\
\hline $\mathrm{H}$ & -0.21236900 & 3.85137100 & -0.46704700 \\
\hline C & -1.72768000 & 3.76274100 & 0.97424400 \\
\hline $\mathrm{H}$ & -2.47275100 & 3.13454400 & 1.46447800 \\
\hline C & -1.66050200 & 5.19156300 & 1.42296400 \\
\hline $\mathrm{H}$ & -2.62497300 & 5.70068500 & 1.28661800 \\
\hline $\mathrm{H}$ & -1.42195700 & 5.25530800 & 2.49393100 \\
\hline $\mathrm{H}$ & -0.89505300 & 5.75001900 & 0.87472800 \\
\hline $\mathrm{H}$ & -6.05017700 & 0.62674900 & 0.92022600 \\
\hline C & -2.54384200 & -1.49048700 & 3.89851100 \\
\hline $\mathrm{H}$ & -3.43799100 & -1.02329100 & 4.32768400 \\
\hline $\mathrm{H}$ & -2.83386300 & -2.48345100 & 3.53544100 \\
\hline $\mathrm{H}$ & -1.81672700 & -1.62223300 & 4.70842400 \\
\hline $\mathrm{C}$ & -1.51951500 & 0.75415900 & 3.32222700 \\
\hline $\mathrm{H}$ & -2.38063300 & 1.32185500 & 3.69495400 \\
\hline $\mathrm{H}$ & -0.82519300 & 0.62413900 & 4.16068300 \\
\hline $\mathrm{H}$ & -1.01599500 & 1.35591000 & 2.56097500 \\
\hline C & -4.19719400 & -3.22683900 & -1.96767400 \\
\hline $\mathrm{H}$ & -5.27581600 & -3.03306500 & -1.92868900 \\
\hline $\mathrm{H}$ & -3.99461600 & -3.81996700 & -2.86741100 \\
\hline $\mathrm{H}$ & -3.94329600 & -3.83624400 & -1.09337000 \\
\hline $\mathrm{C}$ & -3.77677000 & -1.10515900 & -3.26322500 \\
\hline $\mathrm{H}$ & -3.22082300 & -0.16542500 & -3.33362000 \\
\hline $\mathrm{H}$ & -3.55070000 & -1.69674400 & -4.15791900 \\
\hline $\mathrm{H}$ & -4.84595800 & -0.86606000 & -3.29338500 \\
\hline $\mathrm{N}$ & 2.14740200 & 3.05257300 & -0.11022800 \\
\hline O & 2.78880600 & 4.09168500 & -0.21715200 \\
\hline $\mathrm{C}$ & -0.98283900 & 2.70189900 & -2.82711400 \\
\hline $\mathrm{H}$ & -1.15324400 & 2.40466500 & -3.86823200 \\
\hline $\mathrm{H}$ & -1.74275600 & 3.44107500 & -2.55090400 \\
\hline $\mathrm{H}$ & 0.00159100 & 3.17385900 & -2.76549600 \\
\hline
\end{tabular}

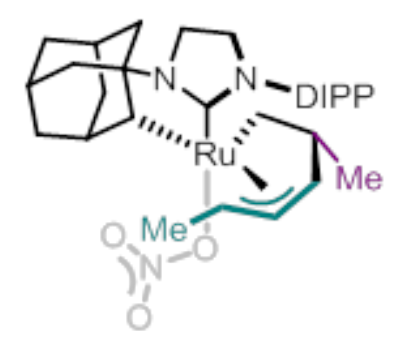

$16 d$

SCF Energy (M06, n-octanol): $\quad-1731.697313$

SCF Energy (B3LYP, gas-phase): $\quad-1731.374154$

Enthalpy Correction:

0.790833

Free-Energy Correction:

0.685616

O

0.92663900

2.39242800

1.32509800

1.69274000

2. 82068300

$-0.66543900$ 


\begin{tabular}{|c|c|c|}
\hline 0.75949500 & -1.79689400 & 0.70644900 \\
\hline-1.39491300 & -1.44199300 & 0.5002540 \\
\hline 0.15062600 & -0.88570700 & 0.27361300 \\
\hline 0.18384200 & -3.09587300 & 1.05063000 \\
\hline 0.62319600 & -3.50427500 & 1.9650850 \\
\hline 0.34829600 & -3.81772000 & 0.2386290 \\
\hline-1.29390000 & -2.73869600 & 1.20 \\
\hline-1.96453900 & -3.47865400 & 0.76602 \\
\hline-1.57323800 & -2.61108900 & 2.26205 \\
\hline 2.19033800 & -1.56006400 & 0.5311 \\
\hline 2.76928900 & -2.40727400 & -0.6306 \\
\hline 2.17660000 & -2.26225900 & -1.53854 \\
\hline 2.69976400 & -3.47481200 & -0.37468 \\
\hline 4.24734200 & -2.03299900 & -0.86 \\
\hline 4.6 & -2.62717100 & $-1 \cdot 7$ \\
\hline 5.0 & -2.3 & 0.4 \\
\hline 5.000 & -3.416128 & 0.6 \\
\hline 6.11 & -2.09815 & 0.25 \\
\hline 4.48 & -1.51448300 & 1.58 \\
\hline 5.0 & -1.7310 & 2. \\
\hline 3.0 & -1.90 & 1. \\
\hline 2.5 & -1 & 2 . \\
\hline 2.9 & -2.98 & 2 . \\
\hline 4.6 & -0.011295 & 1.2 \\
\hline 4.2 & 0.601185 & 2.08 \\
\hline 5.6 & 0.243 & \\
\hline 3.77 & 0.30 & -0 . \\
\hline 3.8 & 1.3 & -0 . \\
\hline 2.3 & -0 & \\
\hline 2.11971 & 0.412 & 1. \\
\hline 4.35886 & -0.5291 & $-1 \cdot 1$ \\
\hline $3.85785^{\circ}$ & -0.29883300 & -2.13 \\
\hline 5.416811 & -0.263 & $-1 \cdot 3$ \\
\hline-2.6 & -0.769 & 0.5 \\
\hline-3.6 & -1.04 & -0 \\
\hline-4.9 & -0.415 & -0.2 \\
\hline-5.67324 & -0.605681 & -0.9 \\
\hline-5.174509 & 0.44268000 & 0.80 \\
\hline-4.20860800 & 0.658920 & 1.7863 \\
\hline-4.4 & 1.3 & 2.6 \\
\hline-2.9 & 0.050 & 1.7 \\
\hline-3.4 & -2.046 & -1.5 \\
\hline-2.37075 & -2.275081 & -1.5 \\
\hline-1.98306400 & 0.23011100 & 2.89 \\
\hline-1.01841400 & -0.20794900 & 2.6192 \\
\hline-6.1 & 0.926580 & 0.8 \\
\hline-2.5 & -0.52 & 4.1 \\
\hline-3.4 & -0 . & 4.4 \\
\hline-2.701213 & -1.58209600 & 3.92 \\
\hline-1.78518100 & -0.46191300 & $4.95^{\circ}$ \\
\hline-1.71867 & 1.705673 & 3.2 \\
\hline-2.63749 & 2.224 & 3.5 \\
\hline-1.0 & 1.765 & 4.0 \\
\hline-1.26341 & 2.241535 & 2.40729 \\
\hline-4.19747200 & -3.36256700 & -1.224492 \\
\hline-5.28097200 & -3.19487900 & -1.21145 \\
\hline-3.98253100 & -4.10650100 & -2.00084 \\
\hline-3.92139700 & -3.7921000 & -0.255467 \\
\hline-3.85516200 & -1.5052330 & -2.893836 \\
\hline & -0.5623210 & -3.132357 \\
\hline & -2.2329030 & -3.67170 \\
\hline-4 . & 47450 & -2.9557 \\
\hline 1.57759300 & 9 & 0.560602 \\
\hline
\end{tabular}




$\begin{array}{lrrr}\mathrm{O} & 2.05311500 & 4.24062300 & 0.96514700 \\ \mathrm{Ru} & 0.55924800 & 0.88136100 & -0.46815400 \\ \mathrm{C} & 1.25194700 & 0.25632300 & -2.40917300 \\ \mathrm{H} & 1.89337800 & -0.61641700 & -2.43471300 \\ \mathrm{H} & 1.81252900 & 1.14397700 & -2.72697000 \\ \mathrm{C} & -0.07046000 & 0.07077800 & -3.14783200 \\ \mathrm{C} & -0.93812000 & 0.63702000 & -2.04420500 \\ \mathrm{H} & -0.29257000 & -1.00141900 & -3.22885100 \\ \mathrm{C} & -0.99991200 & 2.04810100 & -1.79321500 \\ \mathrm{H} & -1.76354100 & 0.04157500 & -1.67657200 \\ \mathrm{C} & -1.57631800 & 2.54462900 & -0.64987200 \\ \mathrm{H} & -2.22282600 & 1.89675500 & -0.06479700 \\ \mathrm{C} & -1.51137100 & 3.98453500 & -0.23943800 \\ \mathrm{H} & -1.17440700 & 4.08500300 & 0.79876600 \\ \mathrm{H} & -0.83113400 & 4.55744100 & -0.87679800 \\ \mathrm{H} & -2.50841200 & 4.44417000 & -0.29545600 \\ \mathrm{H} & -0.41645800 & 2.72510400 & -2.41464700 \\ \mathrm{C} & -0.19161900 & 0.69829300 & -4.54117000 \\ \mathrm{H} & 0.51558200 & 0.22642800 & -5.23429100 \\ \mathrm{H} & -1.20215500 & 0.57669400 & -4.95254000 \\ \mathrm{H} & 0.03700700 & 1.77027800 & -4.52036100 \\ & & \end{array}$

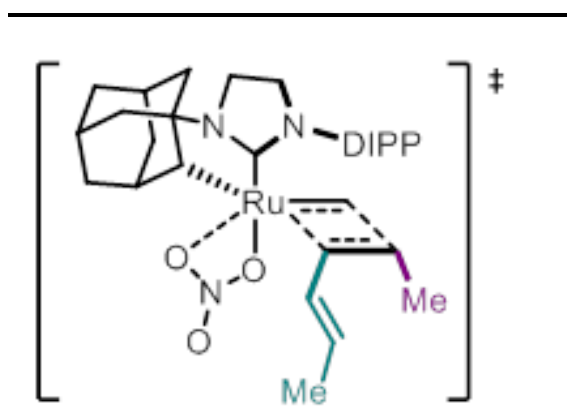

\section{7-TS}

SCF Energy (M06, n-octanol):

$-1731.669122$

SCF Energy (B3LYP, gas-phase): Enthalpy Correction:

Free-Energy Correction:
$-1731.347965$
0.787243
0.680175 


\begin{tabular}{|c|c|c|c|}
\hline & 5.76607200 & 0.29309300 & 0.96188800 \\
\hline & 3.77231700 & 0.43275700 & 0.06335000 \\
\hline & 3.78487000 & 1.52680300 & 0.036892 \\
\hline & 2.36733800 & -0.07565100 & 0.432924 \\
\hline & 2.16421800 & 0.21933100 & 1.471995 \\
\hline & 4.23892000 & -0.11744600 & -1.299495 \\
\hline & 3.56853000 & 0.23102300 & -2.094674 \\
\hline & 5.24209000 & 0.26586100 & -1.532462 \\
\hline & -2.52230800 & -1.21018500 & 0.437385 \\
\hline & -3.42633500 & -1.49817400 & -0.613484 \\
\hline & -4.70527800 & -0.93010000 & -0.558243 \\
\hline & -5.41394300 & -1.12975800 & -1.356869 \\
\hline & -5.08911400 & -0.12207500 & 0.508386 \\
\hline & -4.20691000 & 0.10396100 & 1.560960 \\
\hline & -4.52848100 & 0.70617600 & 2.405968 \\
\hline & -2.91671700 & -0.44310900 & 1.560654 \\
\hline & -3.07807700 & -2.46823800 & -1.742370 \\
\hline & -1.99723500 & -2.63981500 & -1.706376 \\
\hline & -2.03065900 & -0.26085400 & 2.794256 \\
\hline & -1.04769300 & -0.68834000 & 2.579207 \\
\hline & 0.45412100 & 0.01851900 & -2.268465 \\
\hline & -0.01450800 & -0.89989100 & -2.644332 \\
\hline & -1.36620100 & 1.88159100 & -0.776055 \\
\hline & -1.96486300 & 1.40912500 & -0.004739 \\
\hline & -1.28134300 & 1.19317000 & -2.022582 \\
\hline & -1.89641300 & 0.30143400 & -2.063982 \\
\hline & -1.17901800 & 1.97107300 & -3.326228 \\
\hline & -1.10442400 & 1.30008800 & -4.186930 \\
\hline & -2.07681200 & 2.59061100 & -3.455475 \\
\hline & -0.31083300 & 2.63569500 & -3.338400 \\
\hline & 0.96626200 & 0.57401900 & -3.064577 \\
\hline & 1.56368700 & 3.08966500 & 0.228166 \\
\hline & 2.01233600 & 4.19539100 & 0.485898 \\
\hline & -3.41792200 & -1.93924400 & -3.148715 \\
\hline & -3.09618200 & -2.66328900 & -3.906346 \\
\hline & -4.49561100 & -1.78904700 & -3.279887 \\
\hline & -2.92095700 & -0.98830400 & -3.364063 \\
\hline & -3.78310500 & -3.82454300 & -1.518381 \\
\hline & -3.47328300 & -4.55075400 & -2.279370 \\
\hline & -3.55599000 & -4.24567900 & -0.533118 \\
\hline & -4.87201600 & -3.71325000 & -1.583693 \\
\hline & -2.62587400 & -1.03236000 & 3.993058 \\
\hline & -3.59403600 & -0.61394800 & 4.292661 \\
\hline & -2.78424400 & -2.09163100 & 3.759282 \\
\hline & -1.95380300 & -0.97028200 & 4.856921 \\
\hline & -1.79572700 & 1.21383000 & 3.171271 \\
\hline & -1.26311900 & 1.75310700 & 2.383842 \\
\hline & -2.73765700 & 1.73537300 & 3.380833 \\
\hline & -1.18236600 & 1.27045500 & 4.078003 \\
\hline & -6.08420000 & 0.31434300 & 0.529338 \\
\hline & -1.23699100 & 3.33248100 & -0.676835 \\
\hline & -0.71418900 & 3.85072100 & -1.476633 \\
\hline & -1.71582800 & 4.05750700 & 0.349831 \\
\hline & -2.22440200 & 3.54182200 & 1.164670 \\
\hline & -1.57985800 & 5.54324800 & 0.483903 \\
\hline & -0.98352000 & 5.79715800 & 1.370865 \\
\hline & -1.08517100 & 5.98328500 & -0.387943 \\
\hline & -2.55823300 & 6.02759900 & 0.609645 \\
\hline
\end{tabular}




\section{Diene Homodimerization}

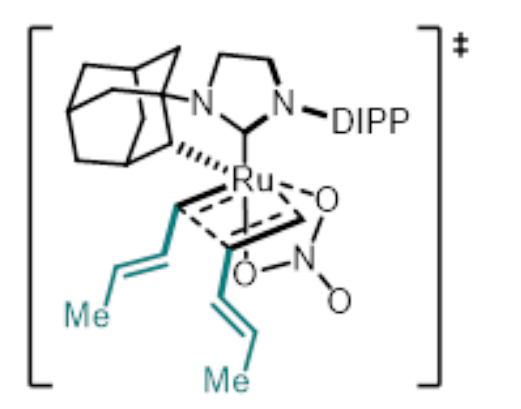

\section{8-TS}

SCF Energy (M06, n-octanol): $\quad-1809.032243$

SCF Energy (B3LYP, gas-phase): $\quad-1808.752596$

Enthalpy Correction:

0.823264

Free-Energy Correction:

0.710459

0.51751000

1.83017000

1.34455300

1.05589800

$-1.09197100$

0.04057200

0.69227300

1. 07714600

1.09315000

$-0.83727300$

$-1.29956500$

$-1.26195800$

2. 43952000

3. 06762400

2. 44043400

3.09456000

4.49579300

4.92958000

5.35988700

5.43508900

6.38158800

4.73529400

5.35175900

3. 32058200

2. 86404400

3.39241400

4. 65691100

4.24673700

5.66826800

3.77098100

3.71838600

2. 36871600

2.06784300

4. 42144500

3. 84590200

5.43261400

$-2.35174800$

$-2.45153100$

$-3.69672200$

$-3.79305300$

$-4.81673100$

$-4.71502200$

$-5.60157300$

$-3.49300500$

$-1.28265600$

$-0.36946900$
0.73213600

0.75681400

2.55614200

$-1.67406400$

$-1.79158800$

$-1.04801000$

$-2.98217900$

$-3.11089700$

$-3.79344600$

$-2.91612900$

$-3.83475100$

$-2.69165800$

$-1.26074100$

$-2.12114600$

$-2.04898700$

$-3.17732300$

$-1.62814300$

$-2.25846300$

$-1.72010800$

$-2.76337600$

$-1.37974000$

$-0.84492900$

$-0.89863400$

$-1.37865900$

$-0.80187200$

$-2.42400500$

0.61839400

1.26120900

0.98840900

0.70347800

1.74655800

0.21145800

0.79272400

$-0.16716500$

$-0.10023600$

0.20749200

$-1.75892900$

$-2.38223100$

$-2.37778900$

$-2.83797600$

$-1.80815900$

$-1.25415800$

$-0.84427600$

$-1.22661500$

$-3.12623700$

$-2.86046600$
$-0.33376200$

$-2.42321700$

$-1.30238800$

1.19900600

0.77535500

0.54837500

1. 74895600

2.76415900

1.12577800

1.70555700

1.33634100

2. 69185600

0.94919100

$-0.17913500$

$-1.07620300$

0.12622200

$-0.48775900$

$-1.27535300$

0.78560600

1.12527400

0.56976700

1.89162000

2. 79890100

2.21637900

3. 03187300

2. 55292000

1. 40249800

2.19344700

1.18201400

0.12778200

$-0.20343000$

0.52811700

1. 42123100

$-0.96869500$

$-1.89480300$

$-1.18067400$

0.08214800

$-1.18848000$

$-1.82918100$

$-2.80809600$

$-1.23024100$

0.04160900

0.51650000

0.72718900

$-1.83706300$

$-1.29790400$ 


\begin{tabular}{|c|c|c|}
\hline-3.45503200 & -0.70567800 & 2.16268900 \\
\hline-2.40390400 & -0.59176900 & 2.44789000 \\
\hline-0.99606900 & 0.73749000 & -1.80207700 \\
\hline-1.46448600 & -0.22713500 & -1.94932600 \\
\hline-0.66482300 & 1.66033700 & 0.87953100 \\
\hline-1.37566000 & 1.14280400 & 1.52761200 \\
\hline-1.66445300 & 1.65196000 & -0.87930900 \\
\hline-2.54421800 & 1.22608400 & -0.40091800 \\
\hline-0.59593100 & 1.17636200 & -2.71417600 \\
\hline-0.36707100 & 3.01978400 & 1.31862600 \\
\hline 0.28932300 & 3.61971700 & 0.69030300 \\
\hline-0.80648000 & 3.52854200 & 2.48636600 \\
\hline-1.46860200 & 2.92168000 & 3.10720700 \\
\hline-0.44420400 & 4.88007400 & 3.02616800 \\
\hline 0.06777100 & 4.79882000 & 3.99529800 \\
\hline 0.21291400 & 5.42363300 & 2.33958700 \\
\hline-1.34123500 & 5.49127800 & 3.19856400 \\
\hline-4.09727300 & -1.73106800 & 3.12327900 \\
\hline-4.01159500 & -1.39141700 & 4.16241000 \\
\hline-5.16269600 & -1.86129400 & 2.89932300 \\
\hline-3.62276000 & -2.71523000 & 3.04613800 \\
\hline-4.13450700 & 0.66608800 & 2.33700700 \\
\hline-5.21026100 & 0.61610000 & 2.13399100 \\
\hline-4.01535900 & 1.01314900 & 3.37015600 \\
\hline-3.70301200 & 1.42456700 & 1.67648200 \\
\hline-1.48493200 & -4.65184600 & -1.69981100 \\
\hline-1.62062700 & -4.95640800 & -0.65578500 \\
\hline-2.37121700 & -4.98094000 & -2.25532500 \\
\hline-0.61822400 & -5.19044400 & -2.10087200 \\
\hline-1.05106900 & -2.76120600 & -3.31588800 \\
\hline-0.17864700 & -3.30457100 & -3.69625800 \\
\hline-1.90511700 & -3.03550100 & -3.94584600 \\
\hline-0.85887000 & -1.69301400 & -3.44771600 \\
\hline-5.77278300 & -1.81434400 & -1.74686600 \\
\hline-1.80026400 & 3.07081800 & -1.27677700 \\
\hline-0.95721700 & 3.49440800 & -1.82001400 \\
\hline-2.88155900 & 3.81942500 & -1.02496200 \\
\hline-3.71428900 & 3.37801500 & -0.47317100 \\
\hline-3.05641800 & 5.24627500 & -1.45821600 \\
\hline-3.93292100 & 5.36279000 & -2.11060000 \\
\hline-3.21883900 & 5.90657300 & -0.59492100 \\
\hline-2.17830500 & 5.60983700 & -2.00223600 \\
\hline 1.94153100 & 2.01920300 & -2.31995000 \\
\hline 2.5664920 & 2.70193600 & -3.11722800 \\
\hline
\end{tabular}

2.70193600

$-3.11722800$

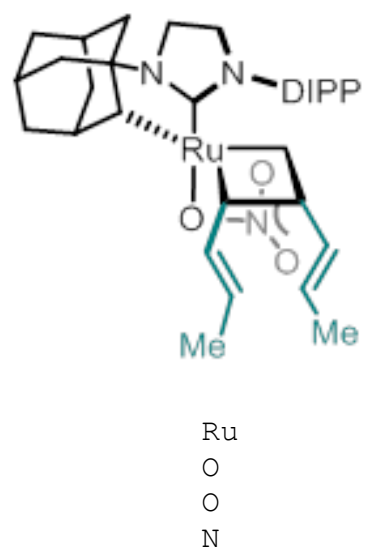

\section{$19 a$}

SCF Energy (M06, n-octanol):

$-1809.041065$

SCF Energy (B3LYP, gas-phase):

Enthalpy Correction:

Free-Energy Correction:
$-1808.758160$

0.824779

0.710981
0.55200400
0.73982300
$-0.27040500$
1. 91898300
1.07530100
2.64016200
1. 33111900
$-1.75903400$
$-2.48933700$
$-1.08557700$
1.10372600 


\begin{tabular}{|c|c|c|}
\hline-1.09368200 & -1.82350700 & 0.70657000 \\
\hline 700 & -1.07973600 & 0.5115280 \\
\hline 0.68918800 & -3.09475000 & 1.57696700 \\
\hline 1.08656100 & -3.29153100 & 2.57650400 \\
\hline 1.06944500 & -3.87040300 & 0.89831900 \\
\hline-0.84023300 & -3.00769100 & 1.5600110 \\
\hline-1.31885300 & -3.89571700 & 1.14057 \\
\hline-1.24806600 & -2.84081000 & 2.5 \\
\hline 2.44232500 & -1.35785800 & 0.8 \\
\hline 3.03911000 & -2.19162200 & -0.33 \\
\hline 2.39278000 & -2.08777600 & $-1 \cdot 21$ \\
\hline 3.06033300 & -3.25544700 & $-0.0567 \varepsilon$ \\
\hline 4.46522900 & -1.70377600 & -0.657455 \\
\hline 4.87593 & -2.31727400 & $-1 \cdot 4$ \\
\hline 5.3 & -1.836 & 0.5 \\
\hline & -2.88 & \\
\hline 6.37453 & -1.49972200 & 0.36 \\
\hline 4.7626 & -0.98685200 & 1.73 \\
\hline 5.39761 & -1.07110400 & 2.62988 \\
\hline 3.34 & -1.511 & 2.0 \\
\hline 2.9 & -0.945 & 2.9 \\
\hline & -2 . & \\
\hline 4. & 0.489 & 1.2 \\
\hline 4.2 & 1.112 & 2.1 \\
\hline 5.692 & 0.86308700 & 1.06 \\
\hline 3.7 & 0.616732 & 0.03 \\
\hline 3.72 & 1.668 & -0.2 \\
\hline 2.3 & 0.12 & 0 . \\
\hline 2.0 & 0.7 & \\
\hline 4.3 & -0.23066 & -1 \\
\hline 3.798 & -0.12473700 & -2.0 \\
\hline 5.40426 & 0.14173600 & $-1 \cdot 32$ \\
\hline-2.37311 & -1.714 & 0. \\
\hline-2.5 & -2.235 & $-1 \cdot 2$ \\
\hline 3. & -2.161 & -1 \\
\hline-3.914 & -2.54225600 & -2.8 \\
\hline-4.87422 & -1.62340200 & -1.1 \\
\hline-4.726225 & -1.16895200 & 0.1 \\
\hline-5.59105 & -0.77965200 & 0.6 \\
\hline-3.484 & -1.212 & 0.7 \\
\hline-1.38 & -2.94 & -1.9 \\
\hline-0.4 & -2.712 & -1 \\
\hline-3.39729 & -0.79058200 & 2.2 \\
\hline-2.33697 & -0.70402700 & 2.49913 \\
\hline-0.90892800 & 0.85431400 & -1.661704 \\
\hline-1. & -0.03130800 & $-1 \cdot 9$ \\
\hline$-0 . \varepsilon$ & 1.67 & 0 . \\
\hline-1 & 1.07 & 1. \\
\hline-1.612667 & 1.72276300 & -0.56383 \\
\hline-2.55691 & 1.22021600 & -0.337538 \\
\hline-0.60245 & 1.424917 & -2.5 \\
\hline-4.02240 & -1.871105 & 3. \\
\hline-3.9046 & -1.599721 & 4.2 \\
\hline-5.094 & -1.976879 & 2.94764 \\
\hline-3.56152400 & -2.85284200 & 2.99582 \\
\hline-4.05706 & 0.57262900 & 2.522933 \\
\hline-5.14191 & 0.536620 & 2.372458 \\
\hline-3.8844 & 0.860533 & 3.566502 \\
\hline-3.65474300 & 1.3649580 & 1.884731 \\
\hline-1.58792800 & -4.4734970 & -1.941206 \\
\hline-1.67519 & 4.840091 & \\
\hline 023 & -4.7648500 & \\
\hline 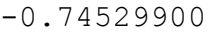 & - & -2.415416 \\
\hline
\end{tabular}




$\begin{array}{lrrr}\mathrm{C} & -1.21473900 & -2.49264600 & -3.46021700 \\ \mathrm{H} & -0.35375500 & -3.00406000 & -3.90555600 \\ \mathrm{H} & -2.09077700 & -2.74125200 & -4.07026300 \\ \mathrm{H} & -1.03861900 & -1.41648400 & -3.53862200 \\ \mathrm{H} & -5.84460000 & -1.57535100 & -1.66865300 \\ \mathrm{~N} & 1.92930000 & 2.30239300 & -2.19296500 \\ \mathrm{O} & 2.46504300 & 3.15920900 & -2.88338300 \\ \mathrm{C} & -1.85910600 & 3.12481500 & -1.05334900 \\ \mathrm{H} & -0.98033300 & 3.67018700 & -1.39384500 \\ \mathrm{C} & -3.06718200 & 3.69178500 & -1.10870100 \\ \mathrm{H} & -3.93557700 & 3.12207400 & -0.76985600 \\ \mathrm{C} & -3.34270700 & 5.08147800 & -1.61045700 \\ \mathrm{H} & -3.81038000 & 5.70078200 & -0.83246300 \\ \mathrm{H} & -2.42225400 & 5.58027100 & -1.93193400 \\ \mathrm{H} & -4.03724700 & 5.06913200 & -2.46174900 \\ \mathrm{C} & -0.37998100 & 2.95486300 & 1.45683400 \\ \mathrm{H} & 0.10819700 & 3.67179000 & 0.79986900 \\ \mathrm{C} & -0.49761500 & 3.24254700 & 2.76434700 \\ \mathrm{H} & -0.98688100 & 2.51930400 & 3.42075600 \\ \mathrm{C} & -0.00599000 & 4.50225500 & 3.41637000 \\ \mathrm{H} & -0.82759900 & 5.05037800 & 3.89870600 \\ \mathrm{H} & 0.72835400 & 4.28489700 & 4.20492400 \\ \mathrm{H} & 0.46713600 & 5.17079100 & 2.68942400 \\ & & \end{array}$

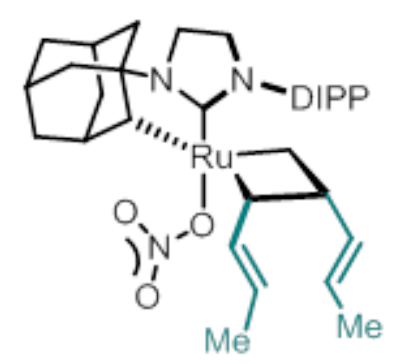

$19 \mathrm{~b}$

SCF Energy (M06, n-octanol): $-1809.045689$

SCF Energy (B3LYP, gas-phase):

$-1808.754534$

Enthalpy Correction:

0.825099

Free-Energy Correction:

0.711503

$$
\begin{array}{r}
0.64175300 \\
2.34637400 \\
1.36474000 \\
1.10980600 \\
-1.06220700 \\
0.08896300 \\
0.70516600 \\
1.17624100 \\
0.97109500 \\
-0.80828900 \\
-1.38549600 \\
-1.09452200 \\
2.47412400 \\
2.83762700 \\
2.07011200 \\
2.83470800 \\
4.22046300 \\
4.46475400 \\
5.27738700 \\
5.32746700 \\
6.27171700 \\
4.91806300 \\
5.67075300 \\
3.53611600 \\
3.27564800 \\
3.56792000
\end{array}
$$

$$
\begin{array}{r}
0.65756400 \\
3.12095900 \\
2.48247600 \\
-1.84133700 \\
-1.84014000 \\
-1.14225300 \\
-3.15631500 \\
-3.38323700 \\
-3.94997200 \\
-2.96750300 \\
-3.84856000 \\
-2.68398000 \\
-1.51454600 \\
-2.30765100 \\
-2.11824400 \\
-3.38607100 \\
-1.87483400 \\
-2.45272400 \\
-2.14287700 \\
-3.21841600 \\
-1.84404400 \\
-1.34822200 \\
-1.53568100 \\
-1.80782400 \\
-1.27449000 \\
-2.88166000
\end{array}
$$

$$
\begin{array}{r}
-0.32618200 \\
0.61112000 \\
-1.25331600 \\
1.06950500 \\
0.76245000 \\
0.49734900 \\
1.57324300 \\
2.53313100 \\
0.86011900 \\
1.69091000 \\
1.40446500 \\
2.71126200 \\
0.64706400 \\
-0.63787900 \\
-1.39950600 \\
-0.42507100 \\
-1.15758900 \\
-2.05885800 \\
-0.06618700 \\
0.15621400 \\
-0.42388200 \\
1.20673400 \\
1.98366700 \\
1.73088600 \\
2.65452300 \\
1.96878900
\end{array}
$$




\begin{tabular}{|c|c|c|}
\hline 4.88445000 & 0.15631200 & 0.86435300 \\
\hline 4.66319900 & 0.75326300 & 1.75829000 \\
\hline 5.87187900 & 0.47579900 & 0.50289200 \\
\hline 3.81242700 & 0.42950500 & -0.2 \\
\hline 3.78631900 & 1.49809900 & -0.44068800 \\
\hline 2.46290500 & -0.01022200 & 0.35611700 \\
\hline 2.28407700 & 0.54401700 & 1.30078800 \\
\hline 4.17100000 & -0.37125300 & -1.49280800 \\
\hline 3.43263900 & -0.18229400 & -2.2 \\
\hline & -0.03849900 & -1.8 \\
\hline 601750 & -1.70409600 & 0.15397 \\
\hline & -2.219872 & $-1 \cdot 1$ \\
\hline .840061 & -2.10540800 & -1.71 \\
\hline .01 & -2.47534400 & -2.72025 \\
\hline & -1.53872800 & -1.0 \\
\hline & -1.09355100 & 0.2 \\
\hline & -0.6 & 0.8 \\
\hline & -1.172052 & 0.9 \\
\hline 1. & -2.96935200 & -1.92 \\
\hline .500029 & -2.60784100 & -1.5743360 \\
\hline 皮 & -0.75278500 & 2.3 \\
\hline-2. & -0.6 & 2.5 \\
\hline & 0.6 & -1.9 \\
\hline-1 . & -0.284579 & -2.22 \\
\hline-0. & 1.610980 & 0.53 \\
\hline-1.51136 & 0.95508700 & 1.19 \\
\hline 1. 48295200 & 1.52602200 & -0.96 \\
\hline-2.4 & 0.9751 & $-0 . \varepsilon$ \\
\hline-0 & 1.19 & -2 . \\
\hline-3 & -1.836963 & 3.2 \\
\hline & -1.566002 & 4.3 \\
\hline-4.96529 & -1.944349 & 3.12 \\
\hline 3.429428 & -2.81750000 & 3.127 \\
\hline-3 & $0.60^{\circ}$ & 2.6 \\
\hline-5 . & 0.5 & 2.5 \\
\hline-3 & 0.8950 & 3.7 \\
\hline 8017101 & 1.40220200 & 2.024 \\
\hline-1.547702 & -4.48428500 & -1.62 \\
\hline-1.44850600 & -4.70504100 & -0.5592 \\
\hline-2 & -4.895097 & -1.960 \\
\hline D. & -5.0 & -2. \\
\hline 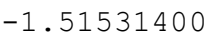 & -2.75703700 & -3.4 \\
\hline-0 & -3.19821000 & -3.90558 \\
\hline-2.38364 & -3.24576100 & -3.9058 \\
\hline-1.542692 & -1.69788500 & -3.72206 \\
\hline-5. & -1.460986 & -1.4650180 \\
\hline 1 & 3.41 & -0 . \\
\hline 2. & 4.52 & -1.03 \\
\hline-1.7 & 2.871138 & -1.60752200 \\
\hline-0.82109 & 3.449333 & -1.8361820 \\
\hline-2.9260270 & 3.33747700 & -1.92160100 \\
\hline-3.80468 & 2.730166 & -1.6911440 \\
\hline 9087800 & 4.659370 & -2.58590 \\
\hline 543 & 4.527448 & -3.542454 \\
\hline-3.83164 & 5.29789800 & -1.96219000 \\
\hline-2.2599080 & 5.20158800 & -2.78163000 \\
\hline-0.7184120 & $2.930170 \mathrm{C}$ & 1.14297500 \\
\hline 63974 & 3.736526 & 0.5083680 \\
\hline-0.91711200 & 3.178863 & 2.4494720 \\
\hline 1.27525100 & 2.37143900 & 3.0913870 \\
\hline 65839600 & 4.49478300 & 3.1191340 \\
\hline 0.10176700 & 4.39176900 & 3.9059460 \\
\hline-0.30246500 & 5.24404 & 2 \\
\hline
\end{tabular}




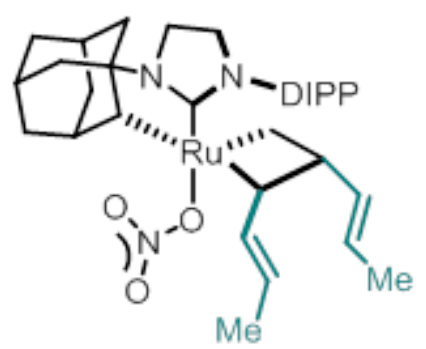

$19 \mathrm{c}$

SCF Energy (M06, n-octanol): SCF Energy (B3LYP, gas-phase): Enthalpy Correction:

Free-Energy Correction:

\author{
$-1809.042001$ \\ $-1808.756902$ \\ 0.825033 \\ 0.711698
}

$$
\begin{aligned}
& 0.66333300 \\
& 1.52521000 \\
& \text { 1. } 49659200 \\
& 1.19761900 \\
& -0.99139100 \\
& 0.13984300 \\
& 0.83893600 \\
& \text { 1. } 39595300 \\
& 1.03880700 \\
& -0.66165700 \\
& -1.25600400 \\
& -0.87808100 \\
& \text { 2. } 54607600 \\
& 3.01213700 \\
& \text { 2. } 26628700 \\
& \text { 3. } 08381100 \\
& \text { 4. } 38026200 \\
& 4.69623800 \\
& 5.41359200 \\
& 5.53051900 \\
& 6.39726700 \\
& \text { 4. } 95377600 \\
& 5.68553100 \\
& \text { 3. } 58557700 \\
& 3.25603700 \\
& \text { 3. } 67626300 \\
& \text { 4. } 82807200 \\
& 4.52909800 \\
& 5.80388400 \\
& \text { 3. } 78571500 \\
& \text { 3. } 72074100 \\
& 2.43508800 \\
& \text { 2. } 18240500 \\
& \text { 4. } 24660900 \\
& \text { 3. } 53133700 \\
& 5.21298200 \\
& -2.30456400 \\
& -3.36039800 \\
& -4.63044200 \\
& -5.45543800 \\
& -4.85723800 \\
& -3.81965200 \\
& -4.01515500 \\
& -2.53062100 \\
& -3.17344800 \\
& -2.09672800 \\
& -1.45303000 \\
& -0.51378400 \\
& -0.08570000 \\
& 0.67091500 \\
& 2.15555200 \\
& \text { 2. } 69298900 \\
& -2.16008400 \\
& -2.08909400 \\
& -1.31396000 \\
& -3.52877900 \\
& -4.26758700 \\
& -3.70902600 \\
& -3.53418100 \\
& -4.04811200 \\
& -3.99296900 \\
& -1.59275800 \\
& -1.36420400 \\
& -0.76136000 \\
& -2.33080500 \\
& -0.65280500 \\
& -0.49362800 \\
& -1.53712200 \\
& -2.49573700 \\
& -1.04869500 \\
& -1.77644600 \\
& -2.40828800 \\
& -2.49600600 \\
& -2.70279500 \\
& -3.46213900 \\
& -0.41921600 \\
& -0.56778400 \\
& 0.08597000 \\
& 0.47488500 \\
& 1.42677900 \\
& -0.25759500 \\
& -0.47069900 \\
& 0.70330200 \\
& \text { 1. } 35069000 \\
& 1.22582500 \\
& -1.73558900 \\
& -1.61783400 \\
& -1.27444800 \\
& -1.16658800 \\
& -1.07815700 \\
& -1.25870800 \\
& -1.13812300 \\
& -1.60575200 \\
& -1.93587700 \\
& -1.98959100 \\
& -1.88349300 \\
& -2.09857300 \\
& 0.83599100
\end{aligned}
$$

\section{S68}


$-1.19153300$

$-1.25731900$

$-0.45556300$

0.48745600

$-2.17127000$

$-1.88747800$

$-1.28454100$

$-0.70202700$

$-2.07417600$

$-2.67867000$

$-2.18970700$

$-3.22652900$

$-1.88950100$

$-1.55669300$

$-5.84834300$

$-1.82086500$

$-2.73255300$

$-1.99259200$

$-1.01359600$

$-1.18089700$

$-2.07444400$

$-0.39961800$

$-0.84033000$

$-3.78589400$

$-4.87339300$

$-3.58473400$

$-3.38348100$

$-3.76104600$

$-3.32982900$

$-3.55434600$

$-4.84891900$

1.78142700

2. 26537400

$-1.34090700$

$-0.43860100$

$-2.42118800$

$-3.31432600$

$-2.51841200$

$-2.74409000$

$-3.32635900$

$-1.58449400$
1.45576700
1.60408000
$-0.02429400$
1. 51706600
1.05886500
0.69557400
2.67613100
3.54113400
2. 78056000
1. 92146100
4.00325100
4.36513500
3.78550900
4.81612900
$-0.80449400$
$-3.13097700$
$-2.96515000$
$-4.00814300$
$-3.37061400$
$-0.68135600$
$-0.39755200$
$-0.93941300$
0.19251000
$-3.31592900$
$-3.30307600$
$-3.58792000$
$-4.10693900$
$-0.87217200$
0.11761700
$-1.14481200$
$-0.78885500$
3.01722000
4.11564700
3. 02879600
3.62501700
3.55602600
2.93828000
4.96326800
4.97824200
5.51661200
5.51310700
0.32262700
$-1.26268500$
$-2.59249200$
$-2.66724800$
$-1.51121700$
0.66513600
1.13117800
0.82723600
2. 21495200
2. 50932400
3.07451400
3.11883700
4.10912200
2.70470000
1.46976900
3.48566700
4.07143500
2.85085300
4.18766800
3.57750000
4. 14653500
4.30203500
3.01566100
$-2.53470000$
$-2.39481800$
$-3.57776400$
$-1.89230200$
$-3.15129000$
$-2.97414400$
$-4.19270000$
$-3.04700900$
0.90832600
1.15273900
$-1.74220800$
$-1.61432700$
$-2.32446300$
$-2.44402800$
$-2.84312900$
$-3.91845500$
$-2.34504900$
$-2.68549500$ 


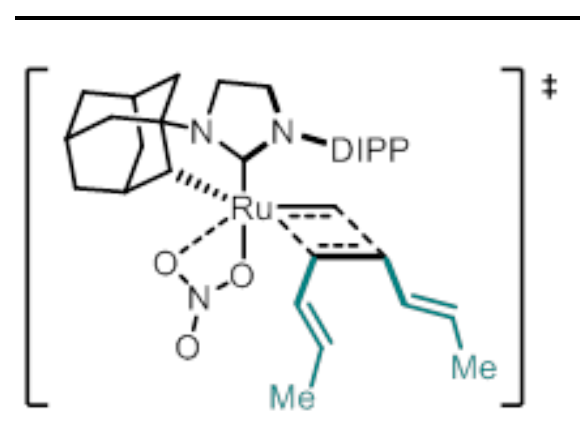

\section{0-TS}

SCF Energy (M06, n-octanol): $\quad-1809.026229$

SCF Energy (B3LYP, gas-phase): $\quad-1808.746274$

Enthalpy Correction:

0.823052

Free-Energy Correction:

0.711103

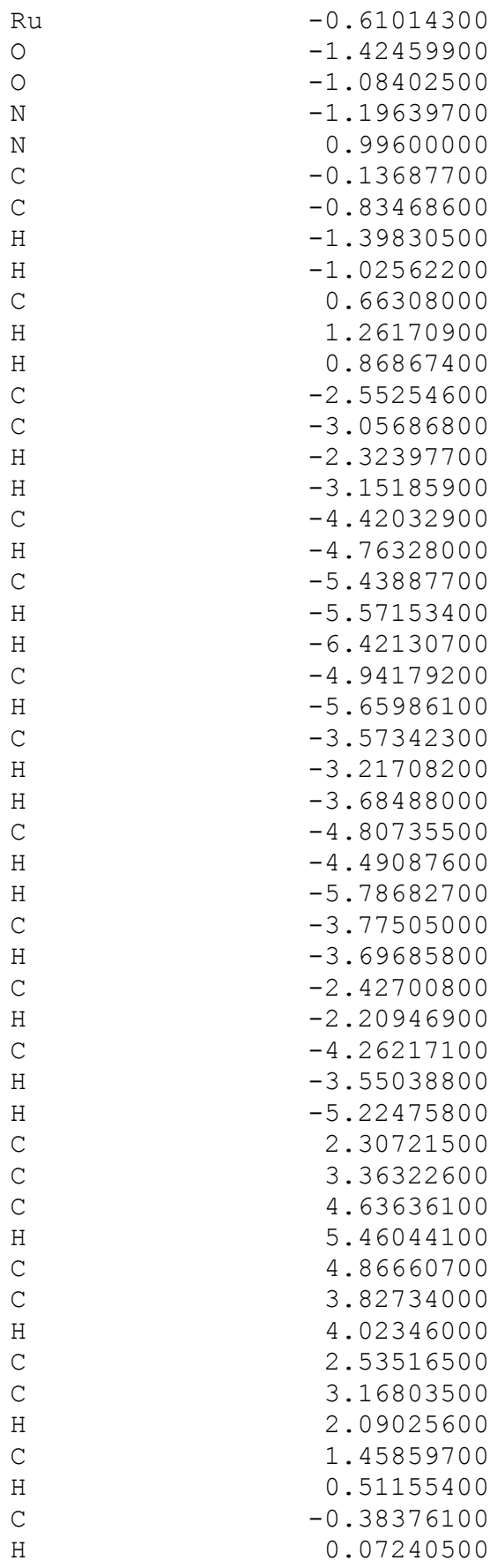

$$
\begin{aligned}
& 0.69661300 \quad 0.12091100 \\
& 2.74746600-0.14055000 \\
& 1.69034900-2.00908500 \\
& -2.12649200 \quad 0.29747400 \\
& -2.05994500 \quad 0.30177600 \\
& -1.28472200 \quad 0.20503900 \\
& -3.49413600 \quad 0.66617000 \\
& -4.23328800 \quad 0.09011100 \\
& -3.67250300 \quad 1.73394400 \\
& -3.50398500 \quad 0.34251000 \\
& -4.02223800 \quad 1.09483700 \\
& -3.96133300-0.63405900 \\
& \begin{array}{ll}
-1.56914300 & 0.32853300
\end{array} \\
& -1.37431600 \quad 1.77988400 \\
& -0.78843800 \quad 2.34412600 \\
& -2.35189700 \quad 2.27528800 \\
& -0.65416400 \quad 1.76329900 \\
& -0.50770300 \quad 2.79639400 \\
& -1.52279100 \quad 0.99755400 \\
& -2.48860900 \quad 1.50616800 \\
& -1.03158000 \quad 0.98378100 \\
& -1.74102600 \quad-0.44638500 \\
& -2.36604300 \quad-0.99388200 \\
& -2.46469100 \quad-0.41625300 \\
& -2.65690100-1.43691800 \\
& -3.43890200 \quad 0.08359100 \\
& -0.36938100 \quad-1.14177400 \\
& -0.49877300 \quad-2.18529100 \\
& 0.12926800-1.16361100 \\
& 0.50664300-0.38348300 \\
& 1.47633300-0.88416100 \\
& -0.23256700-0.42784500 \\
& -0.47877000 \quad-1.47716100 \\
& 0.71278000 \quad 1.06455800 \\
& 1.34199000 \quad 1.61228900 \\
& 1.24284100 \quad 1.06323800 \\
& -1.70411900 \quad-0.17219000 \\
& -1.57931200 \quad 0.76199200 \\
& -1.24896600 \quad 0.28056700 \\
& -1.13837300 \quad 0.97918900 \\
& -1.06430100 \quad-1.07973200 \\
& -1.23844100-1.98907200 \\
& -1.12222900-3.05130800 \\
& -1.57558700 \quad-1.56448800 \\
& -1.85946100 \quad 2.25071200 \\
& -1.85977900 \quad 2.44594000 \\
& -1.84361400 \quad-2.61684800 \\
& -2.03258000 \quad-2.10479300 \\
& 0.83032700 \quad 1.99276500 \\
& 0.11919200 \quad 2.69262400
\end{aligned}
$$




\begin{tabular}{|c|c|c|c|}
\hline C & 1.47290400 & 1.63145500 & -0.18843000 \\
\hline $\mathrm{H}$ & 2.08190700 & 0.79484700 & -0.51124800 \\
\hline C & 1.32896800 & 1.76313300 & 1.23968200 \\
\hline $\mathrm{H}$ & 1.93193400 & 1.05311700 & 1.79882200 \\
\hline $\mathrm{H}$ & -0.85064400 & 1.67730300 & 2.50819300 \\
\hline N & -1.49522400 & 2.74999200 & -1.43254700 \\
\hline O & -1.92503200 & 3.71639200 & -2.04355300 \\
\hline C & 3.81132500 & -0.80233500 & 3.16801200 \\
\hline $\mathrm{H}$ & 3.54598100 & -1.00251700 & 4.21256500 \\
\hline $\mathrm{H}$ & 4.90522900 & -0.81748300 & 3.10274600 \\
\hline $\mathrm{H}$ & 3.48068400 & 0.21197600 & 2.92351400 \\
\hline $\mathrm{C}$ & 3.71965800 & -3.25595500 & 2.61563100 \\
\hline $\mathrm{H}$ & 3.52039900 & -3.48636400 & 3.66908400 \\
\hline $\mathrm{H}$ & 3.27339700 & -4.04738200 & 2.00410800 \\
\hline $\mathrm{H}$ & 4.80462000 & -3.29677800 & 2.46176000 \\
\hline C & 1.80607800 & -3.10768300 & -3.43297000 \\
\hline $\mathrm{H}$ & 2.72533700 & -2.96652500 & -4.01356600 \\
\hline $\mathrm{H}$ & 1.95501800 & -3.98207300 & -2.78851500 \\
\hline $\mathrm{H}$ & 0.99897000 & -3.33745500 & -4.13839800 \\
\hline $\mathrm{C}$ & 1.22098300 & -0.64534400 & -3.55549900 \\
\hline $\mathrm{H}$ & 0.90362800 & 0.24473700 & -3.00609900 \\
\hline $\mathrm{H}$ & 2.12215100 & -0.39698800 & -4.12947900 \\
\hline $\mathrm{H}$ & 0.43164900 & -0.88984800 & -4.27578900 \\
\hline $\mathrm{H}$ & 5.86019600 & -0.80078100 & -1.43248400 \\
\hline C & 1.51191600 & 2.78735700 & -1.08556000 \\
\hline $\mathrm{H}$ & 0.93863800 & 3.66917900 & -0.81114000 \\
\hline $\mathrm{C}$ & 2.23619100 & 2.81608100 & -2.21739800 \\
\hline $\mathrm{H}$ & 2.81616500 & 1.93412600 & -2.49215000 \\
\hline C & 2.30768200 & 3.98223900 & -3.15652400 \\
\hline $\mathrm{H}$ & 1.95503800 & 3.70024100 & -4.15827400 \\
\hline $\mathrm{H}$ & 1.69244500 & 4.81789500 & -2.80773000 \\
\hline $\mathrm{H}$ & 3.34020600 & 4.33957700 & -3.27601900 \\
\hline C & 1.17164200 & 3.08698700 & 1.88397500 \\
\hline $\mathrm{H}$ & 0.50484100 & 3.79267300 & 1.39311700 \\
\hline C & 1.79853000 & 3.43792600 & 3.01490300 \\
\hline $\mathrm{H}$ & 2.45116800 & 2.71216700 & 3.50502600 \\
\hline C & 1.68396300 & 4.77913300 & 3.67914700 \\
\hline $\mathrm{H}$ & 2.66246900 & 5.27326500 & 3.75228200 \\
\hline $\mathrm{H}$ & 1.00888400 & 5.44289800 & 3.12895500 \\
\hline $\mathrm{H}$ & 1.30460600 & 4.68286400 & 4.70599400 \\
\hline
\end{tabular}

\section{Alkene-First Pathway B}

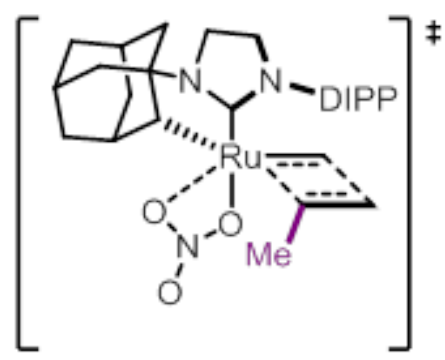
36-TS
SCF Energy (M06, n-octanol):
SCF Energy (B3LYP, gas-phase):
Enthalpy Correction:
Free-Energy Correction:

$-1615.013610$

$-1614.634107$

0.721613

0.623964

$\begin{array}{lrrr}\mathrm{Ru} & 0.61699400 & 0.83955000 & -0.63388500 \\ \mathrm{O} & 1.87044700 & 2.57518200 & -1.21720100 \\ \mathrm{O} & 1.24257100 & 2.59584100 & 0.86606200 \\ \mathrm{~N} & 0.62189900 & -1.75827000 & 0.63963700 \\ \mathrm{~N} & -1.50979900 & -1.31840400 & 0.38056900\end{array}$




$\begin{array}{rrrr}\mathrm{H} & -2.03787400 & -0.05342700 & 4.77832000 \\ \mathrm{C} & -1.67529500 & 1.94866600 & 2.90327700 \\ \mathrm{H} & -1.10048300 & 2.34922900 & 2.06431900 \\ \mathrm{H} & -2.54577600 & 2.59376500 & 3.07133500 \\ \mathrm{H} & -1.04026700 & 2.01432000 & 3.79415800 \\ \mathrm{H} & -6.10780900 & 1.34711400 & 0.43710200 \\ \mathrm{H} & -0.96578300 & 1.95493100 & -3.04629200 \\ \mathrm{C} & -0.92254600 & 3.74152100 & -0.97724300 \\ \mathrm{H} & -1.86036100 & 4.28024100 & -1.17659000 \\ \mathrm{H} & -0.53587200 & 4.10527600 & -0.02221600 \\ \mathrm{H} & -0.20930500 & 3.99863900 & -1.76479000\end{array}$

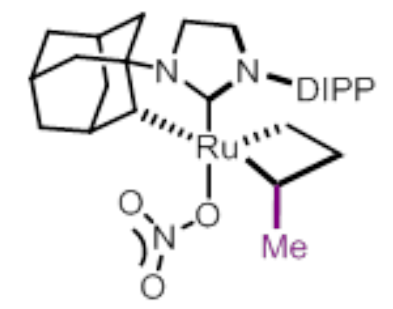

\section{$37 a$}

SCF Energy (M06, n-octanol): $\quad$-1615.037139

SCF Energy (B3LYP, gas-phase): $\quad-1614.650609$

Enthalpy Correction: $\quad 0.724438$

Free-Energy Correction: $\quad 0.626095$

\begin{tabular}{|c|c|c|c|}
\hline $\mathrm{Ru}$ & 0.69815300 & 0.88115700 & -0.43060500 \\
\hline O & 2.14320300 & 2.72654900 & 1.04008100 \\
\hline O & 2.00018800 & 2.47568600 & -1.12864900 \\
\hline $\mathrm{N}$ & 0.47054800 & -1.85324300 & 0.47556100 \\
\hline $\mathrm{N}$ & -1.62053700 & -1.27828200 & 0.18486200 \\
\hline C & -0.33203400 & -0.81067000 & 0.14043000 \\
\hline C & -0.22602300 & -3.13896900 & 0.54282100 \\
\hline $\mathrm{H}$ & 0.10051000 & -3.73264000 & 1.40097500 \\
\hline $\mathrm{H}$ & -0.04829700 & -3.72659800 & -0.36883200 \\
\hline C & -1.68337300 & -2.68165900 & 0.65625800 \\
\hline $\mathrm{H}$ & -2.36949400 & -3.26784200 & 0.03995200 \\
\hline $\mathrm{H}$ & -2.04308200 & -2.71216400 & 1.69230600 \\
\hline C & 1.91720100 & -1.70048000 & 0.30935300 \\
\hline $\mathrm{C}$ & 2.35791100 & -2.10982400 & -1.12205300 \\
\hline $\mathrm{H}$ & 1.77064000 & -1.54531700 & -1.85748500 \\
\hline $\mathrm{H}$ & 2.14740800 & -3.17688300 & -1.28399500 \\
\hline $\mathrm{C}$ & 3.86207300 & -1.83208500 & -1.30981400 \\
\hline $\mathrm{H}$ & 4.15929300 & -2.12580700 & -2.32518300 \\
\hline $\mathrm{C}$ & 4.65874900 & -2.65500000 & -0.27650100 \\
\hline $\mathrm{H}$ & 4.49264000 & -3.72992100 & -0.43740500 \\
\hline $\mathrm{H}$ & 5.73503000 & -2.47597900 & -0.40087600 \\
\hline $\mathrm{C}$ & 4.22282800 & -2.25511300 & 1.14846000 \\
\hline $\mathrm{H}$ & 4.78765400 & -2.84049500 & 1.88595100 \\
\hline $\mathrm{C}$ & 2.71556700 & -2.54561700 & 1.32931900 \\
\hline $\mathrm{H}$ & 2.39310000 & -2.29351300 & 2.34830600 \\
\hline $\mathrm{H}$ & 2.52454400 & -3.61864900 & 1.17877400 \\
\hline $\mathrm{C}$ & 4.49124000 & -0.75121000 & 1.35697100 \\
\hline $\mathrm{H}$ & 4.21454600 & -0.44958400 & 2.37601300 \\
\hline $\mathrm{H}$ & 5.56465500 & -0.54488700 & 1.24356600 \\
\hline $\mathrm{C}$ & 3.68699600 & 0.08153200 & 0.32207200 \\
\hline $\mathrm{H}$ & 3.89417600 & 1.13837600 & 0.49442800 \\
\hline C & 2.19499900 & -0.20552900 & 0.55497600 \\
\hline $\mathrm{H}$ & 1.92816300 & 0.04327500 & 1.59382800 \\
\hline $\mathrm{C}$ & 4.12356800 & -0.32681300 & -1.09921900 \\
\hline $\mathrm{H}$ & 3.57852800 & 0.26857900 & -1.84168100 \\
\hline $\mathrm{H}$ & 5.19145800 & -0.10951400 & -1.23702200 \\
\hline $\mathrm{C}$ & -2.83924700 & -0.51407800 & 0.23665600 \\
\hline $\mathrm{C}$ & -3.68511900 & -0.49665700 & -0.89734200 \\
\hline $\mathrm{C}$ & -4.87775600 & 0.23458500 & -0.81928700 \\
\hline $\mathrm{H}$ & -5.53903600 & 0.27143600 & -1.68021300 \\
\hline
\end{tabular}




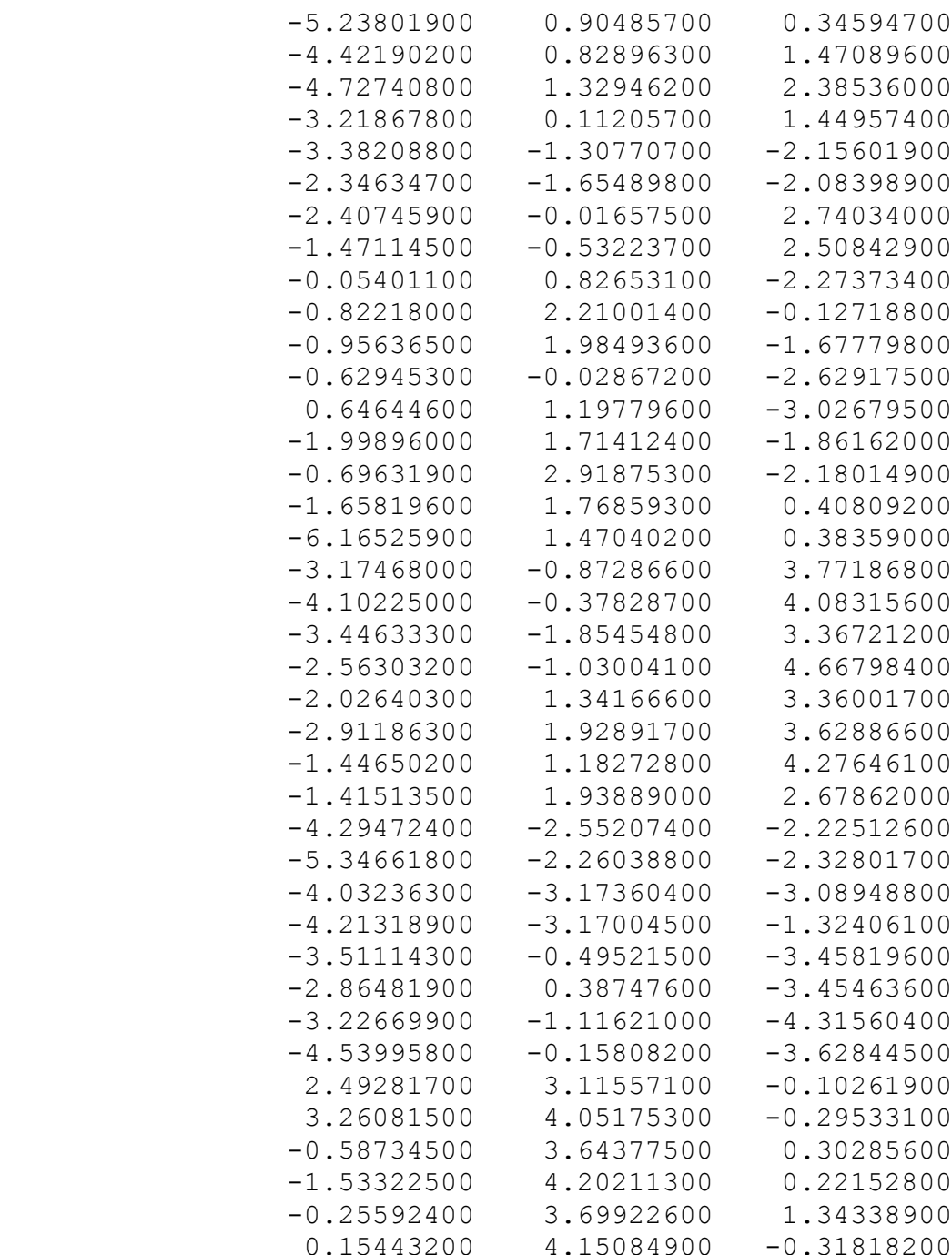

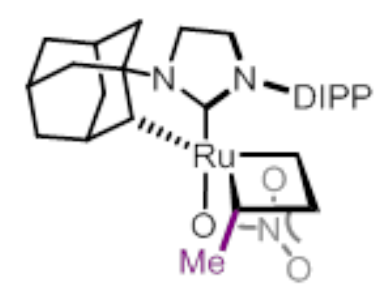

$37 b$

SCF Energy (M06, n-octanol): SCF Energy (B3LYP, gas-phase): Enthalpy Correction:

Free-Energy Correction:

-1615.034796
-1614.650622
0.723936
0.625105

$\begin{array}{lrrr}\mathrm{Ru} & -0.60430700 & 1.12534700 & -0.07794400 \\ \mathrm{O} & -2.15107300 & 1.67384600 & 1.99556400 \\ \mathrm{O} & -1.80214100 & 2.94312000 & 0.25407700 \\ \mathrm{~N} & -0.48655700 & -1.66816300 & -0.83560500 \\ \mathrm{~N} & 1.60677000 & -1.15385500 & -0.43614400 \\ \mathrm{C} & 0.32828600 & -0.66077700 & -0.41793700 \\ \mathrm{C} & 0.19309700 & -2.95711200 & -0.98562100 \\ \mathrm{H} & -0.09927100 & -3.45973700 & -1.91161300 \\ \mathrm{H} & -0.04255200 & -3.62372800 & -0.14478800 \\ \mathrm{C} & 1.66356200 & -2.52620900 & -0.99147400 \\ \mathrm{H} & 2.30347600 & -3.16481700 & -0.37813800 \\ \mathrm{H} & 2.07600000 & -2.50280400 & -2.00734000\end{array}$




$\begin{array}{lrrr}\mathrm{C} & -0.01975500 & 3.13473300 & -2.27392900 \\ \mathrm{H} & 0.75211800 & 3.83762700 & -2.62492100 \\ \mathrm{H} & -0.46757200 & 2.67565000 & -3.16431900 \\ \mathrm{H} & -0.79309000 & 3.71127700 & -1.75796000\end{array}$

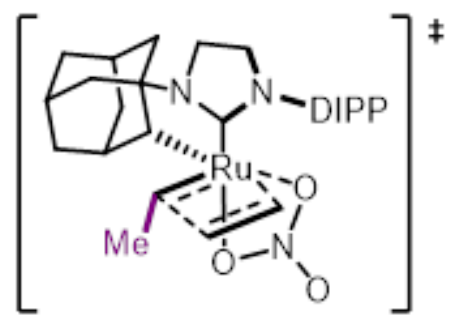

38-TS

SCF Energy (M06, n-octanol):

$-1615.014318$

SCF Energy (B3LYP, gas-phase):

$-1614.635548$

Enthalpy Correction:

0.721930

Free-Energy Correction:

0.623669

\begin{tabular}{|c|c|}
\hline $\mathrm{Ru}$ & -0.54237200 \\
\hline 0 & -1.72189600 \\
\hline 0 & -1.79089400 \\
\hline $\mathrm{N}$ & -0.53311700 \\
\hline $\mathrm{N}$ & 1.58048500 \\
\hline $\mathrm{C}$ & 0.31065900 \\
\hline $\mathrm{C}$ & 0.11067300 \\
\hline $\mathrm{H}$ & -0.19575800 \\
\hline $\mathrm{H}$ & -0.14916700 \\
\hline $\mathrm{C}$ & 1.59666100 \\
\hline $\mathrm{H}$ & 2.20431600 \\
\hline $\mathrm{H}$ & 2.02715300 \\
\hline $\mathrm{C}$ & -1.98218400 \\
\hline $\mathrm{C}$ & -2.44266200 \\
\hline $\mathrm{H}$ & -1.87362800 \\
\hline $\mathrm{H}$ & -2.23181500 \\
\hline $\mathrm{C}$ & -3.95280800 \\
\hline $\mathrm{H}$ & -4.26529800 \\
\hline $\mathrm{C}$ & -4.73893700 \\
\hline $\mathrm{H}$ & -4.57773200 \\
\hline $\mathrm{H}$ & -5.81604100 \\
\hline $\mathrm{C}$ & -4.28403800 \\
\hline $\mathrm{H}$ & -4.84909200 \\
\hline $\mathrm{C}$ & -2.77992800 \\
\hline $\mathrm{H}$ & -2.43257900 \\
\hline $\mathrm{H}$ & -2.62370300 \\
\hline $\mathrm{C}$ & -4.53547100 \\
\hline $\mathrm{H}$ & -4.25280100 \\
\hline $\mathrm{H}$ & -5.60920400 \\
\hline $\mathrm{C}$ & -3.72117200 \\
\hline $\mathrm{H}$ & -3.90168000 \\
\hline $\mathrm{C}$ & -2.23040000 \\
\hline $\mathrm{H}$ & -2.07844000 \\
\hline $\mathrm{C}$ & -4.20936900 \\
\hline $\mathrm{H}$ & -3.70125400 \\
\hline $\mathrm{H}$ & -5.28490800 \\
\hline $\mathrm{C}$ & 2.78068800 \\
\hline $\mathrm{C}$ & 3.01203800 \\
\hline $\mathrm{C}$ & 4.20020800 \\
\hline $\mathrm{H}$ & 4.39309500 \\
\hline $\mathrm{C}$ & 5.14574800 \\
\hline $\mathrm{C}$ & 4.93171400 \\
\hline $\mathrm{H}$ & 5.69311100 \\
\hline $\mathrm{C}$ & 3.75882300 \\
\hline $\mathrm{C}$ & 2.06497800 \\
\hline 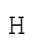 & 1.16016100 \\
\hline
\end{tabular}

$$
\begin{array}{r}
1.16438300 \\
1.30250900 \\
2.91849100 \\
-1.63108200 \\
-1.16288400 \\
-0.64173500 \\
-2.93776600 \\
-3.42643500 \\
-3.60234600 \\
-2.55521100 \\
-3.19215500 \\
-2.58586400 \\
-1.45099100 \\
-1.97780900 \\
-1.46415500 \\
-3.05443600 \\
-1.72350900 \\
-2.11940000 \\
-2.42859400 \\
-3.51583600 \\
-2.26014600 \\
-1.87479900 \\
-2.36415200 \\
-2.18180100 \\
-1.84306900 \\
-3.27046900 \\
-0.34976300 \\
0.05270300 \\
-0.15216500 \\
0.35261700 \\
1.43325900 \\
0.06209900 \\
0.30966500 \\
-0.20563200 \\
0.28511000 \\
-0.00679400 \\
-0.63154100 \\
-0.82812100 \\
-0.32694500 \\
-0.45443100 \\
0.31756600 \\
0.44577800 \\
0.91591500 \\
-0.03298600 \\
-1.63568400 \\
-1.85112200
\end{array}
$$

$-0.06039900$

1.99593900

0.54272400

$-0.81978800$

$-0.46995200$

$-0.43156000$

$-0.97166400$

$-1.90062200$

$-0.13634500$

$-0.97298400$

$-0.32466000$

$-1.98140800$

$-0.66206000$

0.72193100

1.50611700

0.80390200

0.90876700

1.88419300

$-0.21398400$

$-0.17325000$

$-0.08004100$

$-1.57980800$

$-2.38455400$

$-1.76704900$

$-2.75259600$

$-1.72068200$

$-1.62191500$

$-2.60458200$

$-1.49267700$

$-0.49973800$

$-0.54257900$

$-0.78451400$

$-1.84510600$

0.85518300

1.68709000

0.96483400

0.11353900

1.49827100

2. 04535100

3. 10680100

1.25286200

$-0.11627500$

$-0.73200800$

$-0.71523900$

2.38791100

1.81308700 


$\begin{array}{lrrr}\mathrm{C} & 3.61215300 & 0.02439600 & -2.23450800 \\ \mathrm{H} & 2.57029000 & -0.21125000 & -2.47551800 \\ \mathrm{C} & 0.99355000 & 2.07176100 & 1.33636200 \\ \mathrm{H} & 1.61951500 & 1.28687400 & 1.73744800 \\ \mathrm{C} & 0.20058000 & 1.88831000 & -1.63904800 \\ \mathrm{H} & 1.04428800 & 1.44879000 & -2.18584200 \\ \mathrm{C} & 1.35218700 & 2.69074700 & 0.13720700 \\ \mathrm{H} & 2.25764000 & 2.37903700 & -0.37140200 \\ \mathrm{H} & 0.38270700 & 2.61141100 & 2.05130200 \\ \mathrm{C} & 4.50329000 & -1.04716400 & -2.90088600 \\ \mathrm{H} & 4.35191600 & -1.05254900 & -3.98696600 \\ \mathrm{H} & 5.56427000 & -0.84640100 & -2.71032800 \\ \mathrm{H} & 4.28499300 & -2.05124800 & -2.52156800 \\ \mathrm{C} & 3.92683500 & 1.40809800 & -2.83346500 \\ \mathrm{H} & 4.97820500 & 1.68350400 & -2.69458700 \\ \mathrm{H} & 3.73254500 & 1.40229400 & -3.91227800 \\ \mathrm{H} & 3.31339800 & 2.19694000 & -2.38655100 \\ \mathrm{C} & 2.71454900 & -2.98563300 & 2.76494300 \\ \mathrm{H} & 3.02643700 & -3.55135500 & 1.87934400 \\ \mathrm{H} & 3.60393800 & -2.83501100 & 3.38826400 \\ \mathrm{H} & 2.00900400 & -3.60349000 & 3.33273500 \\ \mathrm{C} & 1.61893700 & -0.89021700 & 3.66066000 \\ \mathrm{H} & 0.95981400 & -1.53414000 & 4.25411600 \\ \mathrm{H} & 2.47019000 & -0.61860000 & 4.29578000 \\ \mathrm{H} & 1.06258500 & 0.02081000 & 3.42445100 \\ \mathrm{H} & 6.05975900 & 0.70037400 & 1.69902400 \\ \mathrm{H} & 0.99513100 & 3.69421200 & -0.07328900 \\ \mathrm{~N} & -2.19463600 & 2.44839300 & 1.67504000 \\ \mathrm{O} & -2.97622500 & 3.05985500 & 2.38455300 \\ \mathrm{C} & -0.37696000 & 3.08633700 & -2.33964800 \\ \mathrm{H} & -39563600 & 3.80612300 & -2.64840600 \\ \mathrm{H} & -1.13522700 & 3.59993800 & -1.74249900 \\ \mathrm{H} & & & \\ & & & \\ & & & \\ & & & \end{array}$

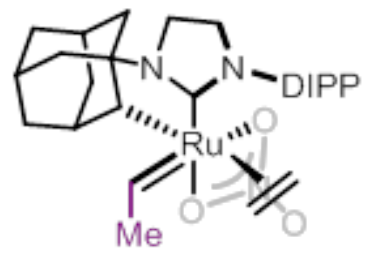

38-C

SCF Energy (M06, n-octanol):

$-1615.012338$

SCF Energy (B3LYP, gas-phase):

$-1614.637651$

Enthalpy Correction:

0.722785

Free-Energy Correction:

0.619274

$$
\begin{array}{r}
-0.52728400 \\
-1.51145400 \\
-1.81219200 \\
-0.57100900 \\
1.54334500 \\
0.28996600 \\
0.04977900 \\
-0.27520600 \\
-0.20829700 \\
1.53926100 \\
2.15445200 \\
1.94918100 \\
-2.00955400 \\
-2.42755700 \\
-1.82269100 \\
-2.23495300 \\
-3.92478100 \\
-4.20819800 \\
-4.76576700
\end{array}
$$

$$
\begin{array}{r}
0.02240700 \\
2.15270700 \\
0.81311400 \\
-0.93345500 \\
-0.52947300 \\
-0.47615600 \\
-1.13192100 \\
-2.07002700 \\
-0.31058900 \\
-1.13664900 \\
-0.55393800 \\
-2.15416600 \\
-0.71541100 \\
0.67981400 \\
1.44964000 \\
0.74179200 \\
0.92412500 \\
1.90837200 \\
-0.17231000
\end{array}
$$




\begin{tabular}{|c|c|c|c|}
\hline $\mathrm{H}$ & -4.62342800 & -3.43562100 & -0.14472800 \\
\hline $\mathrm{H}$ & -5.83392800 & -2.15824900 & 0.00264000 \\
\hline C & -4.35083100 & -1.78963400 & -1.54967600 \\
\hline $\mathrm{H}$ & -4.95358700 & -2.26218700 & -2.33687300 \\
\hline $\mathrm{C}$ & -2.86022000 & -2.12245400 & -1.79347500 \\
\hline $\mathrm{H}$ & -2.54296500 & -1.78681200 & -2.78993300 \\
\hline $\mathrm{H}$ & -2.72135700 & -3.21369300 & -1.75522700 \\
\hline C & -4.57625400 & -0.25979700 & -1.57136100 \\
\hline $\mathrm{H}$ & -4.32684900 & 0.14496900 & -2.56183000 \\
\hline $\mathrm{H}$ & -5.64009600 & -0.04471400 & -1.39658500 \\
\hline $\mathrm{C}$ & -3.70555700 & 0.42152800 & -0.47794400 \\
\hline $\mathrm{H}$ & -3.86911800 & 1.50523000 & -0.50470900 \\
\hline C & -2.23311900 & 0.10631000 & -0.83277600 \\
\hline $\mathrm{H}$ & -2.14343400 & 0.32834600 & -1.90222800 \\
\hline C & -4.15386800 & -0.14031000 & 0.88975300 \\
\hline $\mathrm{H}$ & -3.61222300 & 0.32670000 & 1.71339200 \\
\hline $\mathrm{H}$ & -5.22057400 & 0.07868100 & 1.03889300 \\
\hline $\mathrm{C}$ & 2.78251100 & -0.68668900 & -0.03035100 \\
\hline C & 3.18729200 & -1.05199500 & 1.27677400 \\
\hline C & 4.41703100 & -0.57226000 & 1.74439400 \\
\hline $\mathrm{H}$ & 4.74295200 & -0.82846000 & 2.74881300 \\
\hline C & 5.23064300 & 0.22633700 & 0.94507400 \\
\hline $\mathrm{C}$ & 4.83504000 & 0.54143500 & -0.35153200 \\
\hline $\mathrm{H}$ & 5.48989100 & 1.14218200 & -0.97631700 \\
\hline C & 3.61606400 & 0.08479100 & -0.87033600 \\
\hline C & 2.35138500 & -1.96290500 & 2.17450100 \\
\hline $\mathrm{H}$ & 1.42872000 & -2.20857100 & 1.64082000 \\
\hline $\mathrm{C}$ & 3.26245000 & 0.37199600 & -2.32603700 \\
\hline $\mathrm{H}$ & 2.22003800 & 0.07738300 & -2.47799100 \\
\hline $\mathrm{C}$ & -0.16373700 & 1.75677800 & -1.69084100 \\
\hline $\mathrm{H}$ & 0.06086100 & 1.09931000 & -2.54780000 \\
\hline C & 1.16544800 & 3.01596700 & 0.81240100 \\
\hline $\mathrm{H}$ & 1.83566100 & 3.20651200 & -0.02050700 \\
\hline C & 1.38125100 & 1.98118700 & 1.65309800 \\
\hline $\mathrm{H}$ & 0.77475600 & 1.84282800 & 2.54196400 \\
\hline $\mathrm{H}$ & 2.23195200 & 1.32503800 & 1.52645500 \\
\hline $\mathrm{H}$ & 6.17948400 & 0.59210900 & 1.32849500 \\
\hline C & 3.09272800 & -3.28538100 & 2.46183800 \\
\hline $\mathrm{H}$ & 4.00286800 & -3.11369100 & 3.04831300 \\
\hline $\mathrm{H}$ & 2.45153200 & -3.96468100 & 3.03560100 \\
\hline $\mathrm{H}$ & 3.38839100 & -3.79530800 & 1.53743900 \\
\hline C & 1.93454900 & -1.27853000 & 3.49067000 \\
\hline $\mathrm{H}$ & 1.35151800 & -1.97260300 & 4.10714400 \\
\hline $\mathrm{H}$ & 2.80510800 & -0.96383200 & 4.07795600 \\
\hline $\mathrm{H}$ & 1.31334600 & -0.39848300 & 3.30212200 \\
\hline $\mathrm{C}$ & 4.13613100 & -0.47675900 & -3.27439300 \\
\hline $\mathrm{H}$ & 4.04473400 & -1.54710100 & -3.05830500 \\
\hline $\mathrm{H}$ & 3.84187500 & -0.31345600 & -4.31804800 \\
\hline $\mathrm{H}$ & 5.19554800 & -0.21050900 & -3.17861000 \\
\hline $\mathrm{C}$ & 3.37665400 & 1.86388600 & -2.68709000 \\
\hline $\mathrm{H}$ & 3.03672400 & 2.03078200 & -3.71602600 \\
\hline $\mathrm{H}$ & 2.76459000 & 2.48057500 & -2.02205100 \\
\hline $\mathrm{H}$ & 4.41040900 & 2.22210600 & -2.62294500 \\
\hline $\mathrm{N}$ & -2.04965400 & 2.27227400 & 1.96070500 \\
\hline 0 & -2.74472600 & 2.81837300 & 2.79873900 \\
\hline $\mathrm{H}$ & 0.38199200 & 3.74440500 & 0.98832300 \\
\hline C & -0.35819300 & 3.17705000 & -2.13820600 \\
\hline $\mathrm{H}$ & -0.62008700 & 3.85766000 & -1.32416000 \\
\hline $\mathrm{H}$ & 0.54181900 & 3.55349400 & -2.64910300 \\
\hline $\mathrm{H}$ & -1.16515500 & 3.20681100 & -2.88816900 \\
\hline
\end{tabular}




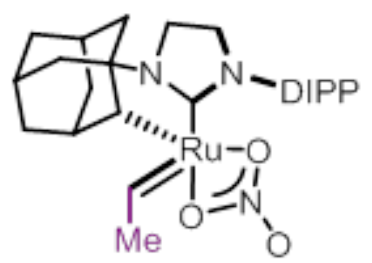

\section{1}

SCF Energy (M06, n-octanol): $\quad-1536.462676$

SCF Energy (B3LYP, gas-phase): $\quad-1536.047585$

Enthalpy Correction:

0.663652

Free-Energy Correction:

0.567398

$$
\begin{aligned}
& 0.83277400-0.85476700 \\
& 2.57262900-0.18989200 \\
& 2.95537500-1.38565500 \\
& -1.75319300 \quad 0.41222200 \\
& -1.26669200 \quad 0.45821500 \\
& -0.83615000 \quad 0.02555700 \\
& -2.74113300 \quad 1.35018400 \\
& -3.74919000 \quad 1.12800900 \\
& -2.49693800 \quad 2.38571500 \\
& -2.59179600 \quad 1.11025500 \\
& -2.61423700 \quad 2.03069400 \\
& -3.36989300 \quad 0.43752800 \\
& -1.25898400 \quad 0.41149600 \\
& -0.39018200 \quad 1.67070700 \\
& 0.39718900 \quad 1.74535400 \\
& -1.00020500 \quad 2.58187100 \\
& 0.24516300 \quad 1.58304100 \\
& 0.85196300 \quad 2.48031300 \\
& -0.87856500 \quad 1.49713300 \\
& -1.49920700 \quad 2.40395400 \\
& -0.44673800 \quad 1.44324300 \\
& \begin{array}{ll}
-1.74248200 & 0.24674700
\end{array} \\
& -2.54091300 \quad 0.18215500 \\
& -2.38465600 \quad 0.35308700 \\
& -3.02583700 \quad-0.51599200 \\
& -3.02176300 \quad 1.24950800 \\
& -0.84499400-1.00770400 \\
& -1.44436400 \quad-1.91517400 \\
& -0.40972700-1.07914800 \\
& 0.27749800 \quad-0.92863900 \\
& 0.90757200-1.82467400 \\
& -0.39332700-0.86285700 \\
& -1.08830500 \quad-1.70802900 \\
& 1.13471400 \quad 0.32494500 \\
& 1.94878900 \quad 0.38987200 \\
& 1.60768100 \quad 0.25587400 \\
& -0.50890800 \quad 0.51749400 \\
& 0.35264000 \quad 1.62049300 \\
& 1.03628600 \quad 1.69397300 \\
& 1.70943800 \quad 2.52654300 \\
& 0.86807100 \quad 0.72500600 \\
& -0.00080700-0.33872800 \\
& -0.13666100 \quad-1.08474000 \\
& -0.70559100 \quad-0.46388200 \\
& 0.52161500 \quad 2.75484900 \\
& 0.04589000 \quad 2.45075900 \\
& -1.68213700-1.62054500 \\
& -1.98506300-1.64050700 \\
& 0.34353100-2.57893900 \\
& 1.19488300-3.27118900 \\
& -0.19398400 \quad 4.03255900 \\
& 0.25493300 \quad 4.40265400 \\
& -0.11416900 \quad 4.82856200 \\
& -1.25824300 \quad 3.85754900
\end{aligned}
$$




$\begin{array}{rrrr}\mathrm{C} & 1.42882800 & 1.99878200 & 3.05479000 \\ \mathrm{H} & 0.63196200 & 2.06182800 & 3.80607200 \\ \mathrm{H} & 2.29917700 & 2.52706200 & 3.46140100 \\ \mathrm{H} & 1.10551400 & 2.52348800 & 2.15190100 \\ \mathrm{C} & 4.21425900 & -2.95174300 & -1.40836600 \\ \mathrm{H} & 4.04751500 & -3.66968000 & -2.22065300 \\ \mathrm{H} & 5.28357500 & -2.70973500 & -1.38899600 \\ \mathrm{H} & 3.97209800 & -3.44747900 & -0.46134400 \\ \mathrm{C} & 3.68534300 & -1.03943400 & -2.98258400 \\ \mathrm{H} & 4.74931800 & -0.79259800 & -3.07267700 \\ \mathrm{H} & 3.44225300 & -1.73226400 & -3.79704000 \\ \mathrm{H} & 3.11502500 & -0.11690600 & -3.13056800 \\ \mathrm{H} & 5.90002200 & 1.41011900 & 0.80238900 \\ \mathrm{~N} & -0.11733200 & 3.45052100 & -0.74516500 \\ \mathrm{O} & 0.08560700 & 4.64153300 & -0.65128800 \\ \mathrm{C} & 0.05720600 & -0.95380400 & -3.26698000 \\ \mathrm{H} & -0.82258900 & -1.20638000 & -3.88297900 \\ \mathrm{H} & 0.90425800 & -0.87252200 & -3.96154500 \\ \mathrm{H} & 0.23422200 & -1.78236700 & -2.57770000\end{array}$

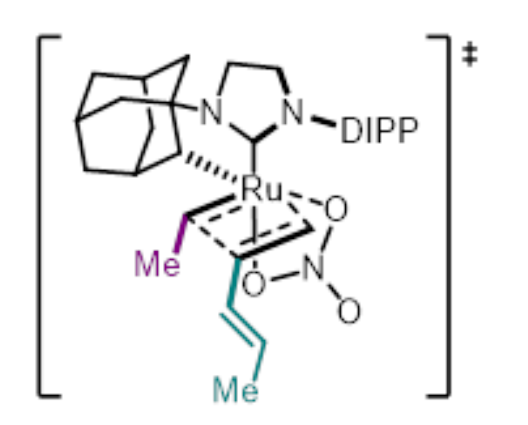

\section{2-TS}

SCF Energy (M06, n-octanol): $\quad-1731.668852$

SCF Energy (B3LYP, gas-phase): $\quad-1731.349296$

Enthalpy Correction: $\quad 0.787022$

Free-Energy Correction: $\quad 0.679921$

$\begin{array}{lr}\mathrm{Ru} & -0.50567800 \\ \mathrm{O} & -1.75878400 \\ \mathrm{O} & -1.39138500 \\ \mathrm{~N} & -0.99016700 \\ \mathrm{~N} & 1.16251400 \\ \mathrm{C} & 0.01168400 \\ \mathrm{C} & -0.59418900 \\ \mathrm{H} & -0.97268800 \\ \mathrm{H} & -0.97897300 \\ \mathrm{C} & 0.93426700 \\ \mathrm{H} & 1.41305300 \\ \mathrm{H} & 1.35917500 \\ \mathrm{C} & -2.38377400 \\ \mathrm{C} & -2.95526100 \\ \mathrm{H} & -2.31494800 \\ \mathrm{H} & -2.94346600 \\ \mathrm{C} & -4.39579900 \\ \mathrm{H} & -4.79075300 \\ \mathrm{C} & -5.27918100 \\ \mathrm{H} & -5.31905800 \\ \mathrm{H} & -6.30945900 \\ \mathrm{C} & -4.70969400 \\ \mathrm{H} & -5.34116200 \\ \mathrm{C} & -3.28303100 \\ \mathrm{H} & -2.86436100 \\ \mathrm{H} & -3.32314800 \\ \mathrm{C} & -4.67838400 \\ \mathrm{H} & -4.30975100 \\ \mathrm{H} & -5.69938200 \\ & \end{array}$

$$
\begin{array}{rr}
0.92899700 & -0.01253400 \\
1.35199000 & 2.04197900 \\
2.89701800 & 0.55596000 \\
-1.78111400 & -0.92961400 \\
-1.74150100 & -0.51424000 \\
-0.99371900 & -0.45787400 \\
-3.17184200 & -1.16294000 \\
-3.53966800 & -2.12049700 \\
-3.82697000 & -0.36941100 \\
-3.06434200 & -1.13938300 \\
-3.85484000 & -0.55606700 \\
-3.08041000 & -2.15052600 \\
-1.35877600 & -0.75402200 \\
-1.92926700 & 0.57077900 \\
-1.60800200 & 1.40152300 \\
-3.02852100 & 0.54321800 \\
-1.42433900 & 0.78656100 \\
-1.85275700 & 1.71735000 \\
-1.85752500 & -0.40037000 \\
-2.95443200 & -0.47001500 \\
-1.50931600 & -0.24739600 \\
-1.26371600 & -1.70532700 \\
-1.56021100 & -2.55354100 \\
-1.81699800 & -1.92654000 \\
-1.44855100 & -2.87289300 \\
-2.91498100 & -1.99117800 \\
0.27731200 & -1.59433500 \\
0.71771700 & -2.53104300 \\
0.65562400 & -1.44355100
\end{array}
$$




\begin{tabular}{|c|c|c|c|}
\hline $\mathrm{C}$ & -3.77115300 & 0.70405300 & -0.40604400 \\
\hline $\mathrm{H}$ & -3.75144900 & 1.79780100 & -0.34306800 \\
\hline C & -2.35696000 & 0.17420300 & -0.71417000 \\
\hline $\mathrm{H}$ & -2.10950100 & 0.51663500 & -1.73544300 \\
\hline $\mathrm{C}$ & -4.36793100 & 0.11176700 & 0.88879000 \\
\hline $\mathrm{H}$ & -3.77972600 & 0.42216100 & 1.75524900 \\
\hline $\mathrm{H}$ & -5.38852000 & 0.49491600 & 1.02926300 \\
\hline C & 2.42536900 & -1.50648800 & 0.13195200 \\
\hline C & 2.55387800 & -1.80358100 & 1.51323000 \\
\hline $\mathrm{C}$ & 3.80271700 & -1.60685800 & 2.11563500 \\
\hline $\mathrm{H}$ & 3.92185500 & -1.81486900 & 3.17485900 \\
\hline C & 4.89866400 & -1.16202400 & 1.38174500 \\
\hline $\mathrm{C}$ & 4.76974300 & -0.93024200 & 0.01620500 \\
\hline $\mathrm{H}$ & 5.63881500 & -0.61624500 & -0.55454400 \\
\hline C & 3.54274500 & -1.10654900 & -0.63801000 \\
\hline C & 1.41382300 & -2.40007500 & 2.34113700 \\
\hline $\mathrm{H}$ & 0.48921400 & -2.30509800 & 1.76564000 \\
\hline C & 3.47519200 & -0.94508500 & -2.15558000 \\
\hline $\mathrm{H}$ & 2.41849600 & -0.93964600 & -2.44327300 \\
\hline C & 1.05015800 & 1.34716900 & 1.38896600 \\
\hline $\mathrm{H}$ & 1.54399800 & 0.45208700 & 1.74248800 \\
\hline C & 0.56254500 & 1.56750200 & -1.45964700 \\
\hline $\mathrm{H}$ & 1.31481900 & 0.95909000 & -1.97088700 \\
\hline C & 1.65786800 & 2.03356100 & 0.26926700 \\
\hline $\mathrm{H}$ & 2.52814100 & 1.53395500 & -0.15067600 \\
\hline $\mathrm{H}$ & 0.63000200 & 1.96770800 & 2.17739500 \\
\hline C & 4.14955200 & -2.14666000 & -2.85426700 \\
\hline $\mathrm{H}$ & 4.04166000 & -2.06945500 & -3.94280200 \\
\hline $\mathrm{H}$ & 5.22118400 & -2.18015400 & -2.62457100 \\
\hline $\mathrm{H}$ & 3.71526800 & -3.10029900 & -2.53537700 \\
\hline C & 4.10029700 & 0.36847400 & -2.66248200 \\
\hline $\mathrm{H}$ & 5.17851600 & 0.40695500 & -2.47074000 \\
\hline $\mathrm{H}$ & 3.95923700 & 0.45623100 & -3.74602900 \\
\hline $\mathrm{H}$ & 3.64506100 & 1.24616400 & -2.19289900 \\
\hline C & 1.66658300 & -3.90455800 & 2.58457600 \\
\hline $\mathrm{H}$ & 1.80666000 & -4.45483800 & 1.64720700 \\
\hline $\mathrm{H}$ & 2.56660400 & -4.05664600 & 3.19214100 \\
\hline $\mathrm{H}$ & 0.82095500 & -4.35306700 & 3.11915200 \\
\hline $\mathrm{C}$ & 1.17911000 & -1.68554000 & 3.68591800 \\
\hline $\mathrm{H}$ & 0.32577700 & -2.14168200 & 4.20054000 \\
\hline $\mathrm{H}$ & 2.04474900 & -1.77038000 & 4.35304300 \\
\hline $\mathrm{H}$ & 0.95465500 & -0.62415700 & 3.55086800 \\
\hline $\mathrm{H}$ & 5.85769700 & -1.01441500 & 1.87110100 \\
\hline $\mathrm{N}$ & -1.93769100 & 2.56027300 & 1.68076900 \\
\hline 0 & -2.57946400 & 3.36391000 & 2.33965400 \\
\hline $\mathrm{C}$ & 1.72218900 & 3.50750300 & 0.27932600 \\
\hline $\mathrm{H}$ & 0.87938800 & 4.01779700 & 0.74249100 \\
\hline C & 2.74676600 & 4.21628700 & -0.21623500 \\
\hline $\mathrm{H}$ & 3.58164300 & 3.68557300 & -0.67945600 \\
\hline C & 2.85911000 & 5.71269400 & -0.16527500 \\
\hline $\mathrm{H}$ & 1.97886600 & 6.16423200 & 0.30402000 \\
\hline $\mathrm{H}$ & 3.74613500 & 6.02759800 & 0.40165700 \\
\hline $\mathrm{H}$ & 2.96457500 & 6.13939700 & -1.17235600 \\
\hline C & 0.21095500 & 2.81541600 & -2.22346000 \\
\hline $\mathrm{H}$ & 1.09640700 & 3.41875600 & -2.46627900 \\
\hline $\mathrm{H}$ & -0.24233600 & 2.50213700 & -3.17696000 \\
\hline $\mathrm{H}$ & -0.50675400 & 3.44771100 & -1.69416200 \\
\hline
\end{tabular}




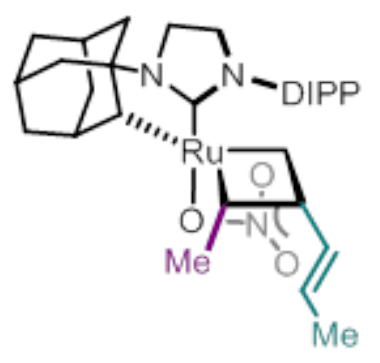

$23 a$

SCF Energy (M06, n-octanol):

$-1731.681022$

SCF Energy (B3LYP, gas-phase):

$-1731.357253$

Enthalpy Correction:

0.788990

Free-Energy Correction:

0.680973

$$
\begin{aligned}
& 0.91370800 \\
& \text { 1. } 79435600 \\
& 2.95101400 \\
& -1.85677700 \\
& -1.79920400 \\
& -1.04105000 \\
& -3.26560500 \\
& -3.72218300 \\
& -3.84106900 \\
& -3.15635800 \\
& -3.91175200 \\
& -3.22700700 \\
& -1.41244900 \\
& -1.92652200 \\
& -1.59439700 \\
& -3.02552700 \\
& -1.38612900 \\
& -1.77470500 \\
& -1.84565100 \\
& -2.94321400 \\
& -1.47302800 \\
& -1.31099500 \\
& -1.62726200 \\
& -1.88928500 \\
& -1.55597400 \\
& -2.98783500 \\
& 0.23084500 \\
& 0.62917800 \\
& 0.63929500 \\
& 0.68681800 \\
& 1.78019700 \\
& 0.11765400 \\
& 0.46309400 \\
& 0.15136700 \\
& 0.49253200 \\
& 0.55914100 \\
& -1.52218500 \\
& -1.67582600 \\
& -1.45697700 \\
& -1.55671100 \\
& -1.12739900 \\
& -1.03087700 \\
& -0.80157200 \\
& -1.23277400 \\
& -2.14827200 \\
& -2.00280200 \\
& -1.20178400 \\
& -1.19152300 \\
& 1.39139400 \\
& 0.60786800 \\
& 1.52582000 \\
& 0.75022900
\end{aligned}
$$




$\begin{array}{rrr}1.60501600 & 1.95511000 & -0.08819800 \\ 2.56098600 & 1.43007700 & -0.17122500 \\ 0.60343300 & 2.16441400 & 1.90638900 \\ 4.05710100 & -2.47422300 & -2.75509500 \\ 3.93852400 & -2.48349500 & -3.84518600 \\ 5.13010600 & -2.51694500 & -2.53389500 \\ 3.60273500 & -3.38695900 & -2.35460700 \\ 4.07247100 & 0.05023500 & -2.77204500 \\ 5.15656600 & 0.06632000 & -2.61223000 \\ 3.90325000 & 0.06153500 & -3.85500900 \\ 3.65800200 & 0.97357900 & -2.35624000 \\ 1.54366600 & -3.65712000 & 2.79715500 \\ 1.58968700 & -4.25719000 & 1.88151500 \\ 2.46163600 & -3.85292300 & 3.36396400 \\ 0.69658800 & -4.01473700 & 3.39444400 \\ 1.27634300 & -1.36851800 & 3.82209800 \\ 0.39324100 & -1.71268000 & 4.37214500 \\ 2.14449100 & -1.52642300 & 4.47239700 \\ 1.16545500 & -0.29354200 & 3.65510200 \\ 5.87220500 & -0.96186300 & 1.82490100 \\ -1.94213700 & 2.90306100 & 1.34642600 \\ -2.49264900 & 3.90082500 & 1.79246000 \\ 1.81879900 & 3.44092900 & 0.01686500 \\ 0.92372600 & 4.03661200 & 0.18919900 \\ 3.01389900 & 4.03199100 & -0.07078200 \\ 3.89992100 & 3.41483200 & -0.23769600 \\ 3.25219600 & 5.51127100 & 0.05116200 \\ 3.72749200 & 5.91566400 & -0.85331500 \\ 2.31532300 & 6.05465500 & 0.21325400 \\ 3.92612300 & 5.73976500 & 0.88833600 \\ 0.41907200 & 2.63785600 & -2.40138600 \\ 1.30842400 & 3.17060400 & -2.76778300 \\ -0.09075100 & 2.20854600 & -3.27320200 \\ -0.24625000 & 3.37514000 & -1.94456600\end{array}$

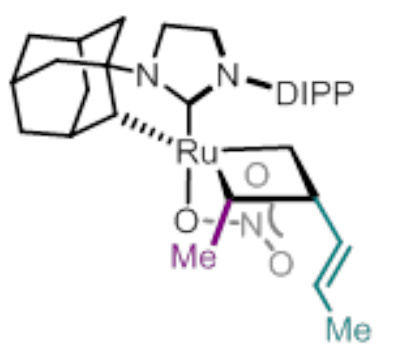

$23 \mathrm{~b}$

SCF Energy (M06, n-octanol): $\quad-1731.679373$

SCF Energy (B3LYP, gas-phase): $\quad-1731.355041$

Enthalpy Correction:

0.789370

Free-Energy Correction: $\quad 0.681747$

$\mathrm{Ru}$
$\mathrm{O}$
$\mathrm{O}$
$\mathrm{N}$
$\mathrm{N}$
$\mathrm{C}$
$\mathrm{C}$
$\mathrm{H}$
$\mathrm{H}$
$\mathrm{C}$
$\mathrm{H}$
$\mathrm{H}$
$\mathrm{C}$
$\mathrm{C}$
$\mathrm{H}$
$\mathrm{H}$

-0.63350600
-2.35346200
-1.54066000
-0.96957800
1.20243800
0.02395900
-0.53505900
-0.96023900
-0.84148100
0.98770400
1.52527300
1.35800800
-2.37004200
-3.04311000
-2.46896600
-3.02957900

0.77904500

2.61875400

2.72204800

$-2.02959800$

$-1.76566500$

$-1.12313800$

$-3.28196100$

$-4.15348800$

$-3.31561300$

$-3.18990400$

$-3.44602600$

$-3.83632800$

$-1.62970600$

$-1.40157900$

$-0.65490900$

$-2.33708700$
-0.18845200
1.41704500
-0.62215200
0.41822400
0.53472900
0.24572300
1.03976500
0.53385700
2.09348900
0.88587400
1.80243600
0.08296600
0.26984900
1.64873000
2.21174400
2.22612900

\section{S83}




$\begin{array}{rrrr}\mathrm{H} & 3.47114300 & 5.89059300 & -1.23544800 \\ \mathrm{H} & 1.87654100 & 6.12542400 & -0.49140400 \\ \mathrm{H} & 3.33924900 & 6.07062500 & 0.51218200 \\ \mathrm{C} & 0.58748600 & 2.13273300 & -2.63541100 \\ \mathrm{H} & 1.49814900 & 2.66158200 & -2.95190000 \\ \mathrm{H} & 0.26956200 & 1.49725500 & -3.47156700 \\ \mathrm{H} & -0.19188500 & 2.87410200 & -2.45076000\end{array}$

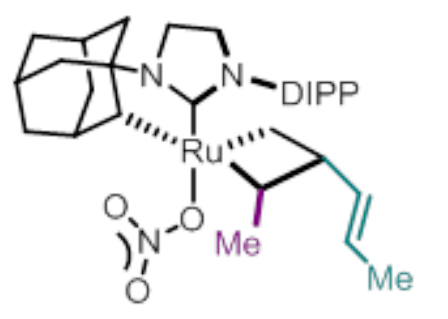

$23 c$

SCF Energy (M06, n-octanol): $\quad-1731.682031$

SCF Energy (B3LYP, gas-phase): $\quad-1731.356683$

Enthalpy Correction:

0.789553

Free-Energy Correction:

0.682847

\begin{tabular}{|c|c|}
\hline $\mathrm{Ru}$ & 0.69147100 \\
\hline 0 & 1.97336300 \\
\hline 0 & 1.71114700 \\
\hline $\mathrm{N}$ & 0.91140200 \\
\hline $\mathrm{N}$ & -1.25556300 \\
\hline $\mathrm{C}$ & -0.05037000 \\
\hline C & 0.40362900 \\
\hline $\mathrm{H}$ & 0.88203600 \\
\hline $\mathrm{H}$ & 0.57719000 \\
\hline C & -1.08521000 \\
\hline $\mathrm{H}$ & -1.73795700 \\
\hline $\mathrm{H}$ & -1.34266800 \\
\hline C & 2.30373900 \\
\hline C & 2.66837200 \\
\hline $\mathrm{H}$ & 1.94836500 \\
\hline $\mathrm{H}$ & 2.58831400 \\
\hline $\mathrm{C}$ & 4.10004700 \\
\hline $\mathrm{H}$ & 4.34395500 \\
\hline C & 5.08681700 \\
\hline $\mathrm{H}$ & 5.05276600 \\
\hline $\mathrm{H}$ & 6.11414600 \\
\hline $\mathrm{C}$ & 4.72723700 \\
\hline $\mathrm{H}$ & 5.42773700 \\
\hline $\mathrm{C}$ & 3.29442800 \\
\hline $\mathrm{H}$ & 3.03064900 \\
\hline $\mathrm{H}$ & 3.23767600 \\
\hline C & 4.80951700 \\
\hline $\mathrm{H}$ & 4.58558500 \\
\hline $\mathrm{H}$ & 5.83153800 \\
\hline C & 3.81325900 \\
\hline $\mathrm{H}$ & 3.89206000 \\
\hline C & 2.39962900 \\
\hline $\mathrm{H}$ & 2.19846800 \\
\hline $\mathrm{C}$ & 4.17661200 \\
\hline $\mathrm{H}$ & 3.49705900 \\
\hline $\mathrm{H}$ & 5.19068400 \\
\hline C & -2.55931800 \\
\hline C & -3.50717200 \\
\hline C & -4.78078700 \\
\hline $\mathrm{H}$ & -5.52240700 \\
\hline $\mathrm{C}$ & -5.11862000 \\
\hline $\mathrm{C}$ & -4.19177500 \\
\hline $\mathrm{H}$ & -4.47430300 \\
\hline . & -2.9048330 \\
\hline
\end{tabular}

0.76042300

1.99360800

2.68576900

$-2.10170600$

$-1.79905300$

$-1.15828700$

$-3.38240300$

$-4.22844700$

$-3.47942600$

$-3.25908800$

$-3.62548800$

$-3.78918800$

$-1.66644300$

$-1.36054600$

$-0.63982800$

$-2.27919400$

$-0.79714600$

$-0.58701300$

$-1.83996000$

$-2.75948600$

$-1.45650900$

$-2.14902700$

$-2.89208300$

$-2.72387800$

$-2.97862300$

$-3.64902000$

$-0.84933700$

$-1.05106300$

$-0.44724600$

0.20129500

1.10727200

$-0.39145700$

$-0.65020700$

0.50122900

1. 26030900

0.92014300

$-1.34712100$

$-0.99424700$

$-0.58942700$

$-0.30634700$

$-0.55663600$

$-0.95994300$

$-0.96082900$

$-1.37598100$
0.05520200

2.13018300

0.07307700

$-0.29793900$

$-0.26851200$

$-0.12731800$

$-0.79161400$

$-0.29095400$

$-1.87263700$

$-0.45840800$

$-1.25436900$

0.46699300

$-0.41381900$

$-1.89221800$

$-2.30061800$

$-2.49126500$

$-1.97618700$

$-3.02592700$

$-1.41214800$

$-2.01385600$

$-1.47131900$

0.05646300

0.45988900

0.12724300

1. 16221800

$-0.46564400$

0.88192000

1. 93785500

0.84311300

0.32064200

0.92303800

0.44378100

1.49441500

$-1.14816800$

$-1.55404600$

$-1.20147100$

0.14336500

$-0.84664000$

$-0.42536600$

$-1.16663500$

0.92417900

1.88103600

2. 92990600

1. 51703800

\section{S85}




\begin{tabular}{|c|c|c|c|}
\hline $\mathrm{C}$ & -3.21735100 & -1.12033900 & -2.34155100 \\
\hline $\mathrm{H}$ & -2.13988400 & -1.27503800 & -2.45811200 \\
\hline $\mathrm{C}$ & -1.95901600 & -1.89741200 & 2.60019800 \\
\hline $\mathrm{H}$ & -0.98400300 & -2.08797500 & 2.14291800 \\
\hline $\mathrm{C}$ & -0.21728100 & 1.32380100 & -1.61887200 \\
\hline $\mathrm{C}$ & -0.97012600 & 1.56848200 & 0.92465500 \\
\hline $\mathrm{C}$ & -1.24161000 & 1.99223900 & -0.58556200 \\
\hline $\mathrm{H}$ & -0.70476400 & 0.61121800 & -2.28543800 \\
\hline $\mathrm{H}$ & 0.34817500 & 2.07968100 & -2.17249700 \\
\hline $\mathrm{H}$ & -2.21107500 & 1.53909100 & -0.81017300 \\
\hline $\mathrm{H}$ & -1.68374300 & 0.80525200 & 1.22328300 \\
\hline $\mathrm{H}$ & -6.11094800 & -0.23517600 & 1.22904400 \\
\hline $\mathrm{C}$ & -2.48105600 & -3.23382600 & 3.17256700 \\
\hline $\mathrm{H}$ & -3.43277700 & -3.09298000 & 3.69834200 \\
\hline $\mathrm{H}$ & -2.64821000 & -3.97939100 & 2.38677400 \\
\hline $\mathrm{H}$ & -1.76240400 & -3.64847300 & 3.88916500 \\
\hline $\mathrm{C}$ & -1.72738700 & -0.88941300 & 3.74206700 \\
\hline $\mathrm{H}$ & -2.65942500 & -0.64727900 & 4.26548600 \\
\hline $\mathrm{H}$ & -1.03674400 & -1.31418100 & 4.47965500 \\
\hline $\mathrm{H}$ & -1.29122600 & 0.04311500 & 3.37489500 \\
\hline $\mathrm{C}$ & -3.93858100 & -2.35593300 & -2.92463200 \\
\hline $\mathrm{H}$ & -5.02703700 & -2.23602100 & -2.86832800 \\
\hline $\mathrm{H}$ & -3.67029700 & -2.49583100 & -3.97854600 \\
\hline $\mathrm{H}$ & -3.68224600 & -3.27344400 & -2.38345000 \\
\hline $\mathrm{C}$ & -3.59857000 & 0.13270900 & -3.15261000 \\
\hline $\mathrm{H}$ & -3.09087700 & 1.02975800 & -2.78615700 \\
\hline $\mathrm{H}$ & -3.31901900 & -0.00435800 & -4.20367600 \\
\hline $\mathrm{H}$ & -4.67789900 & 0.32168500 & -3.12592700 \\
\hline $\mathrm{N}$ & 2.18177000 & 2.89015200 & 1.27396800 \\
\hline $\mathrm{O}$ & 2.79385100 & 3.92563600 & 1.50972700 \\
\hline $\mathrm{C}$ & -1.28335500 & 3.48158700 & -0.79978500 \\
\hline $\mathrm{H}$ & -0.33316000 & 4.00224400 & -0.69282400 \\
\hline $\mathrm{C}$ & -2.38951700 & 4.15489200 & -1.12827900 \\
\hline $\mathrm{H}$ & -3.33022700 & 3.60923400 & -1.23380100 \\
\hline $\mathrm{C}$ & -2.45456000 & 5.63665200 & -1.37217600 \\
\hline $\mathrm{H}$ & -3.15964200 & 6.12315200 & -0.68423700 \\
\hline $\mathrm{H}$ & -1.47455900 & 6.10727800 & -1.24055800 \\
\hline $\mathrm{H}$ & -2.80479400 & 5.85928200 & -2.38960300 \\
\hline $\mathrm{C}$ & -0.90828900 & 2.66852700 & 1.96428200 \\
\hline $\mathrm{H}$ & -1.92204000 & 3.06559500 & 2.12479900 \\
\hline $\mathrm{H}$ & -0.54741800 & 2.27359000 & 2.91822900 \\
\hline $\mathrm{H}$ & -0.27051400 & 3.50641900 & 1.68217600 \\
\hline
\end{tabular}

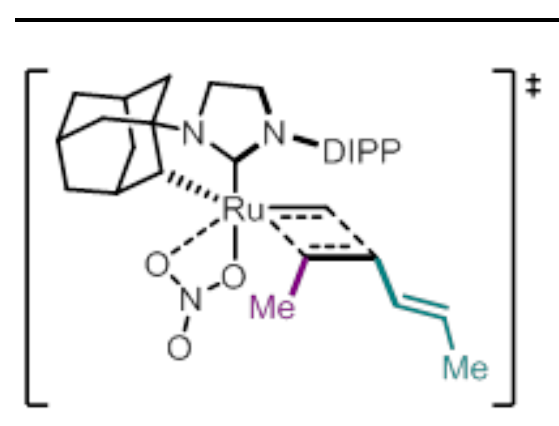

\section{4-TS}

SCF Energy (M06, n-octanol):

SCF Energy (B3LYP, gas-phase):

Enthalpy Correction:

Free-Energy Correction:

-1731.665291
-1731.345432
0.787282
0.680299

Ru
O
O
N
N
C
C

0.63090800
1.68863900
1.44157200
0.91935900
-1.24983500
-0.04653800
0.40403100

$$
\begin{array}{r}
0.78158500 \\
2.65915800 \\
1.33080900 \\
-2.02374400 \\
-1.75690300 \\
-1.11141100 \\
-3.27233700
\end{array}
$$

$$
\begin{array}{r}
0.17293500 \\
0.68321700 \\
2.38753300 \\
-0.49237100 \\
-0.36387300 \\
-0.20937600 \\
-1.05420200
\end{array}
$$




$$
\begin{aligned}
& \begin{array}{lll}
0.91898800 & -4.14482200 & -0.64328000
\end{array} \\
& 0.52284000-3.28523900-2.14707100 \\
& -1.06710100 \quad-3.20017700 \quad-0.63910700 \\
& -1.75402800 \quad-3.53957400 \quad-1.41779600 \\
& \begin{array}{lll}
-1.26488000 & -3.78186300 & 0.27090300
\end{array} \\
& 2.31120200-1.57688900-0.60242900 \\
& 2.66616500-1.20426200 \quad-2.06454200 \\
& 1.93692100-0.48068000-2.44483300 \\
& 2.60766800 \quad-2.09899600 \quad-2.70186000 \\
& 4.08714700-0.61025900-2.11893000 \\
& 4.32475000-0.33715800-3.15594300 \\
& 5.09397700-1.66532200-1.61739900 \\
& 5.07174300-2.55043000-2.26942700 \\
& 6.11543700 \quad-1.26347700 \quad-1.65840800 \\
& \begin{array}{lll}
4.74357800 & -2.05807600 & -0.16710600
\end{array} \\
& \begin{array}{lll}
5.45336000 & -2.81508200 & 0.19227800
\end{array} \\
& 3.31587000 \quad-2.65415900-0.12483100 \\
& \begin{array}{lll}
3.05922700 & -2.96777600 & 0.89563900
\end{array} \\
& 3.27320500-3.54846700 \quad-0.76505500 \\
& \begin{array}{lll}
4.82488700 & -0.80098300 & 0.72483100
\end{array} \\
& \begin{array}{lll}
4.61842400 & -1.06050000 & 1.77155500
\end{array} \\
& \begin{array}{llll}
5.84451100 & -0.39128200 & 0.69155100
\end{array} \\
& 3.80408600 \quad 0.26000900 \quad 0.23420100 \\
& \begin{array}{lll}
3.88103500 & 1.14590900 & 0.87135000
\end{array} \\
& 2.40217100-0.36560400 \\
& 2.27661500-0.74722100 \\
& 4.14258500 \quad 0.64307300 \\
& 3.43655400 \quad 1.40135200 \\
& 5.14558600 \quad 1.08953800 \\
& -2.53665600-1.33625200 \\
& -3.52524200-0.95513200 \\
& -4.77981600-0.56439400 \\
& -5.55284900-0.26052600 \\
& -5.05895900 \\
& -4.09325900 \\
& -4.33111700 \\
& -2.82313100 \\
& -3.29376200 \\
& -2.22128700 \\
& -1.83306300 \\
& -0.88671500 \\
& 0.19540200 \\
& -0.40253500 \\
& -1.24198300 \\
& -1.85998400 \\
& -1.35401900 \\
& -2.07665900 \\
& 0.65984500 \\
& 1.91797300 \\
& \text { 2. } 55013900 \\
& -3.70365600 \\
& -3.47904300 \\
& -4.77731600 \\
& -3.16683200 \\
& -4.03861400 \\
& -3.81126600 \\
& -3.76344700 \\
& -5.12389400 \\
& -2.34998800 \\
& -3.27022200 \\
& -2.56985500 \\
& -1.60204500 \\
& -1.52041500
\end{aligned}
$$




$\mathrm{H}$
$\mathrm{H}$
$\mathrm{H}$
$\mathrm{H}$
$\mathrm{C}$
$\mathrm{H}$
$\mathrm{C}$
$\mathrm{H}$
$\mathrm{C}$
$\mathrm{H}$
$\mathrm{H}$
$\mathrm{H}$
$\mathrm{C}$
$\mathrm{H}$
$\mathrm{H}$
$\mathrm{H}$

$-1.05012300$

$-2.42346300$

$-0.06909100$

$-0.70590800$

$-1.44541600$

$\begin{array}{ll}-0.82465400 & -1.44541600 \\ -6.03670500 & -0.25805200\end{array}$

$-1.21177000$

$-0.42841600$

$-1.99555000$

$-2.77318300$

$-1.90076200$

$-2.83925800$

$-1.09531200$

$-1.71129900$

$-1.12173200$

$-2.10503300$

$-0.81869700$

$-0.41236200$

3.56186800

4.13137900

4.15900800

3.57327800

5.60306100

6.13640400

6.11530600

5.70599700

2.74985100

3.21637600

2.27353500

3.55033400

3.29755000

4.22729600

4.37971000

1.38579600

$-0.80094800$

$-0.30545800$

$-1.70931600$

$-2.20394100$

$-2.10792500$

$-1.90339300$

$-1.57137000$

$-3.18544600$

2. 08713200

2.24928800

3.02157600

1. 87054300

S88 


\section{Alkene Homodimerization}

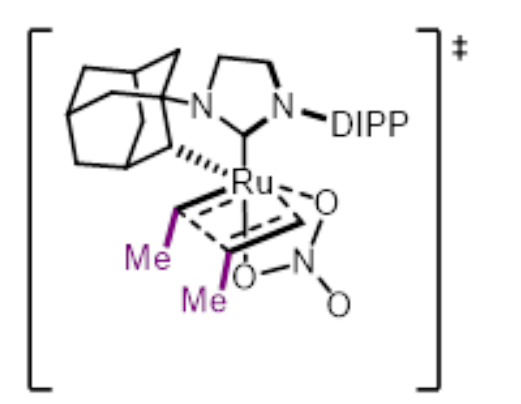

\section{5-TS}

SCF Energy (M06, n-octanol): $\quad-1654.308711$

SCF Energy (B3LYP, gas-phase): $\quad-1653.948443$

Enthalpy Correction:

0.751638

Free-Energy Correction:

0.650159

$$
\begin{array}{r}
-0.56225200 \\
-1.82372800 \\
-1.79192300 \\
-0.58975600 \\
1.53130400 \\
0.26942700 \\
0.03331300 \\
-0.28974800 \\
-0.22674200 \\
1.52256100 \\
2.13203900 \\
1.93690900 \\
-2.03491900 \\
-2.50127300 \\
-1.92239000 \\
-2.30559400 \\
-4.00678000 \\
-4.32281600 \\
-4.80375700 \\
-4.65723100 \\
-5.87835600 \\
-4.34317600 \\
-4.91560200 \\
-2.84345000 \\
-2.49364000 \\
-2.69907900 \\
-4.57336100 \\
-4.28438200 \\
-5.64392400 \\
-3.75027400 \\
-3.91695600 \\
-2.26443100 \\
-2.09124100 \\
-4.24173600 \\
-3.71999000 \\
-5.31358600 \\
2.74503300 \\
2.96291800 \\
4.16468900 \\
4.34717800 \\
5.13624900 \\
4.93389600 \\
5.71376500 \\
3.74865300 \\
1.98229300 \\
1.06298400
\end{array}
$$

$$
\begin{array}{r}
1.07978100 \\
1.32945000 \\
2.87021400 \\
-1.71405200 \\
-1.28779500 \\
-0.74569700 \\
-3.02660700 \\
-3.49223000 \\
-3.70360500 \\
-2.66314800 \\
-3.32845200 \\
-2.66450200 \\
-1.51948000 \\
-2.08454700 \\
-1.60510100 \\
-3.16602300 \\
-1.81464600 \\
-2.23636300 \\
-2.47475600 \\
-3.56481200 \\
-2.29609100 \\
-1.88649300 \\
-2.34407600 \\
-2.20505900 \\
-1.83930100 \\
-3.29622300 \\
-0.35784600 \\
0.06985800 \\
-0.14814100 \\
0.29939600 \\
1.38271200 \\
-0.00244500 \\
0.29491300 \\
-0.29250300 \\
0.17033300 \\
-0.08110400 \\
-0.80652100 \\
-1.04127300 \\
-0.59438400 \\
-0.75213500 \\
0.03451200 \\
0.20002100 \\
0.65771600 \\
-0.22498000 \\
-1.83139400 \\
-1.97925900
\end{array}
$$

$-0.05384200$

1.99864300

0.46489200

$-0.83502200$

$-0.48071900$

$-0.42750200$

$-1.01998900$

$-1.95499100$

$-0.19466600$

$-1.03006900$

$-0.41318700$

$-2.04573000$

$-0.67097300$

0.69633800

1.49502100

0.74257500

0.89482500

1. 85822800

$-0.24752600$

$-0.23960300$

$-0.10705800$

$-1.59698800$

$-2.41504900$

$-1.79719300$

$-2.77210300$

$-1.78490500$

$-1.59347800$

$-2.56356800$

$-1.45793200$

$-0.45027300$

$-0.46137200$

$-0.73841700$

$-1.78640000$

0.88850800

1. 72859400

1.00970700

0.12030400

1.50183400

2. 06528000

3.12443300

1.29196700

$-0.07461100$

$-0.67637400$

$-0.69006500$

2.37052600

1.79742700 


$\begin{array}{lrrr}\mathrm{C} & 3.61666900 & -0.12753100 & -2.20871500 \\ \mathrm{H} & 2.56931400 & -0.31953800 & -2.46409200 \\ \mathrm{C} & 0.94502700 & 1.83946700 & 1.32645700 \\ \mathrm{H} & 1.55957800 & 1.02555100 & 1.68559500 \\ \mathrm{C} & 0.30471800 & 1.80966000 & -1.57315600 \\ \mathrm{H} & 1.15792000 & 1.33856600 & -2.07199100 \\ \mathrm{C} & 1.38260800 & 2.56403700 & 0.18259200 \\ \mathrm{H} & 2.28970900 & 2.18800000 & -0.28339500 \\ \mathrm{H} & 0.40716200 & 2.38555200 & 2.09771800 \\ \mathrm{C} & 4.47550500 & -1.21339600 & -2.89401500 \\ \mathrm{H} & 4.33467400 & -1.18585700 & -3.98116800 \\ \mathrm{H} & 5.54099500 & -1.05552900 & -2.68897200 \\ \mathrm{H} & 4.21823800 & -2.21840400 & -2.54264200 \\ \mathrm{C} & 3.98493400 & 1.25842700 & -2.77110400 \\ \mathrm{H} & 5.04617400 & 1.48889100 & -2.62531200 \\ \mathrm{H} & 3.79146400 & 1.28862200 & -3.84966000 \\ \mathrm{H} & 3.40205600 & 2.05779500 & -2.30266200 \\ \mathrm{C} & 2.56438400 & -3.22582900 & 2.69271100 \\ \mathrm{H} & 2.83263500 & -3.77777300 & 1.78450100 \\ \mathrm{H} & 3.46989700 & -3.14234000 & 3.30517800 \\ \mathrm{H} & 1.83568100 & -3.82441700 & 3.25187200 \\ \mathrm{C} & 1.58732100 & -1.11140900 & 3.67432300 \\ \mathrm{H} & 0.88048900 & -1.73039100 & 4.23868500 \\ \mathrm{H} & 2.45397300 & -0.93232200 & 4.32111500 \\ \mathrm{H} & 1.10229400 & -0.15135100 & 3.47834500 \\ \mathrm{H} & 6.06053500 & 0.37545000 & 1.75078100 \\ \mathrm{~N} & -2.24210800 & 2.46969600 & 1.60987100 \\ \mathrm{O} & -3.01770900 & 3.15010000 & 2.26271900 \\ \mathrm{C} & -0.28073700 & 2.93997200 & -2.37510800 \\ \mathrm{H} & 0.48177500 & 3.62801500 & -2.76675700 \\ \mathrm{H} & -0.77403600 & 2.48928700 & -3.25183200 \\ \mathrm{H} & -1.03503600 & 3.51038900 & -1.82714600 \\ \mathrm{C} & 1.17720800 & 4.06282100 & 0.11944800 \\ \mathrm{H} & 1.36619900 & 4.47653100 & -0.87487700 \\ \mathrm{H} & 0.16307300 & 4.33081200 & 0.42647300 \\ \mathrm{H} & 1.88244900 & 4.54270800 & 0.81172800 \\ & & & \\ & & & \\ & & & \\ & & & \end{array}$

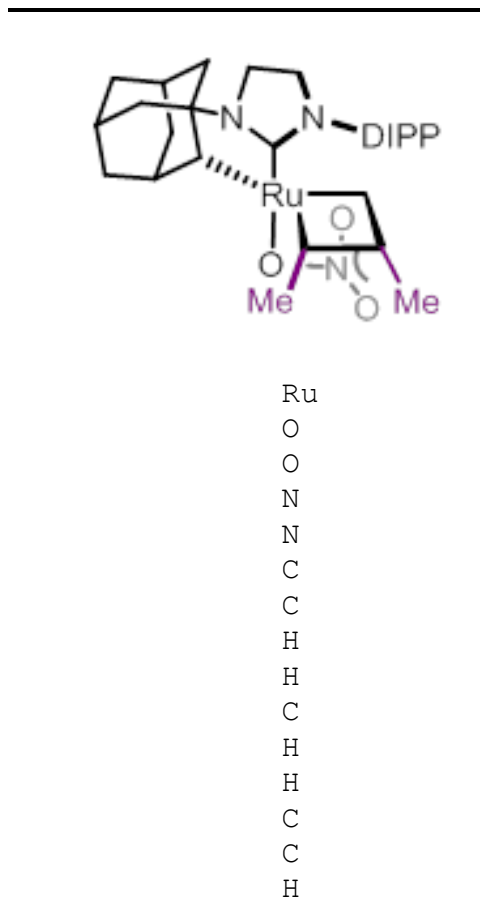

\section{$26 a$}

SCF Energy (M06, n-octanol): SCF Energy (B3LYP, gas-phase): Enthalpy Correction:

Free-Energy Correction:

$$
\begin{array}{r}
-1654.325170 \\
-1653.961117 \\
0.753715 \\
0.652423
\end{array}
$$

$$
\begin{array}{rrr}
-0.60825300 & 1.03891700 & -0.09977800 \\
-2.14195300 & 1.65602900 & 1.97265200 \\
-1.78279300 & 2.88134800 & 0.20178000 \\
-0.56228300 & -1.76795800 & -0.81113400 \\
1.54445300 & -1.30597800 & -0.42009200 \\
0.28014500 & -0.77593800 & -0.40862700 \\
0.08228200 & -3.07781700 & -0.93366600 \\
-0.23285300 & -3.59765000 & -1.84238400 \\
-0.15919600 & -3.71517400 & -0.07184500 \\
1.56117800 & -2.68329800 & -0.96601200 \\
2.19631000 & -3.33226500 & -0.35894600 \\
1.95621300 & -2.67594000 & -1.98912900 \\
-2.00556000 & -1.59492700 & -0.63882200 \\
-2.47727700 & -2.20734900 & 0.70768600 \\
-1.90066400 & -1.75607600 & 1.52487000
\end{array}
$$




$\begin{array}{rrrr}\mathrm{C} & 1.26633900 & 4.00212200 & 0.09385800 \\ \mathrm{H} & 1.84491700 & 4.30359900 & 0.97436100 \\ \mathrm{H} & 1.69995600 & 4.50129200 & -0.77986000 \\ \mathrm{H} & 0.23983700 & 4.35495200 & 0.22261000\end{array}$

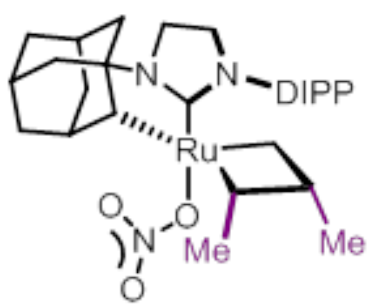

$26 \mathrm{~b}$

SCF Energy (M06, n-octanol):

$-1654.327210$

SCF Energy (B3LYP, gas-phase):

$-1653.960872$

Enthalpy Correction:

0.754214

Free-Energy Correction:

0.652989

\begin{tabular}{l} 
Ru \\
O \\
O \\
N \\
N \\
C \\
C \\
H \\
H \\
C \\
H \\
H \\
C \\
C \\
H \\
H \\
C \\
H \\
C \\
H \\
H \\
C \\
H \\
C \\
H \\
H \\
C \\
H \\
H \\
C \\
H \\
C \\
H \\
C \\
H \\
H \\
C \\
C \\
C \\
H \\
C \\
C \\
H \\
C \\
C \\
H \\
\hline
\end{tabular}

0.71900300
2.19449500
1.99770800
0.53100000
-1.56780400
-0.28688600
-0.14587300
0.18813900
0.04252400
-1.60916800
-2.28485400
-1.97010500
1.97131400
2.36841500
1.75846700
2.15275800
3.86481600
4.13132700
4.69533100
4.52581200
5.76678000
4.30424800
4.89414600
2.80397000
2.51221000
2.61353300
4.57623400
4.33532100
5.64455900
3.73383100
3.94349600
2.25160400
2.02593300
4.12736500
3.55817100
5.18972600
-2.81478500
-3.61392100
-4.84444700
-5.46985700
-5.28648500
-4.51315500
-4.88077500
-3.27575000
-3.22105200
-2.16769000
-2.51313000
0.86789800

2.49947900

2.56062600

$-1.96562600$

$-1.39378100$

$-0.90183600$

$-3.26088200$

$-3.94370500$

$-3.73812900$

$-2.84248800$

$-3.36274900$

$-2.99931300$

$-1.78515300$

$-2.04252700$

$-1.40459100$

$-3.08706800$

$-1.74274300$

$-1.93194200$

$-2.66004900$

$-3.71442700$

$-2.46440800$

$-2.40477800$

$-3.05642800$

$-2.72257300$

$-2.57416300$

$-3.77701400$

$-0.92716500$

$-0.72872300$

$-0.70471300$

$-0.00153400$

1.03452400

$-0.32421600$

$-0.19085800$

$-0.26474300$

0.39613900

$-0.02692500$

$-0.67846600$

$-0.54726600$

0.11302600

0.23447400

0.60540300

0.41964400

0.78043600

$-0.23382400$

$-1.16351400$

$-1.45378600$

$-0.49548900$
$-0.23159000$

1. 47112100

$-0.70761700$

0.34052400

0.11002000

0.12115800

0.25740000

1. 04337500

$-0.71464100$

0.41616700

$-0.26687500$

1. 43964900

0.15691100

$-1.32137200$

$-1.97385800$

$-1.58864700$

$-1.52646600$

$-2.57474100$

$-0.60556600$

$-0.86743600$

$-0.74602100$

0.86504200

1. 52299300

1.06254100

2. 11068300

0.81308400

1. 21131100

2.26394800

1.08060600

0.29219900

0.56069200

0.54904000

1.61851000

$-1.17604500$

$-1.84120800$

$-1.32252100$

0.19781000

$-0.96314400$

$-0.84650400$

$-1.72604900$

0.37741600

1. 51949600

2. 47580600

1. 46193300

$-2.30490900$

$-2.24085700$

2.76183400 


$\begin{array}{lrrr}\mathrm{H} & -1.53258300 & -0.90810800 & 2.50635100 \\ \mathrm{C} & -0.07022400 & 1.08532700 & -2.03866900 \\ \mathrm{H} & -0.62883900 & 0.28482000 & -2.52389300 \\ \mathrm{C} & -0.82264900 & 2.10139000 & 0.29822100 \\ \mathrm{H} & -1.63908900 & 1.55231800 & 0.75989900 \\ \mathrm{C} & -1.01213400 & 2.12727000 & -1.27564000 \\ \mathrm{H} & -2.02479500 & 1.73962300 & -1.41995000 \\ \mathrm{H} & 0.61154600 & 1.58906300 & -2.73145700 \\ \mathrm{C} & -3.25845600 & -1.54006000 & 3.62248200 \\ \mathrm{H} & -2.67264800 & -1.78741500 & 4.51559100 \\ \mathrm{H} & -4.22894700 & -1.15405100 & 3.95591900 \\ \mathrm{H} & -3.44801300 & -2.46829100 & 3.07172300 \\ \mathrm{C} & -2.26170600 & 0.77925800 & 3.58985800 \\ \mathrm{H} & -3.19929800 & 1.24274800 & 3.91749000 \\ \mathrm{H} & -1.68691600 & 0.53107900 & 4.48949600 \\ \mathrm{H} & -1.69439700 & 1.52372700 & 3.02564200 \\ \mathrm{C} & -4.04679400 & -2.44099100 & -2.57590700 \\ \mathrm{H} & -3.95010900 & -3.17308300 & -1.76661100 \\ \mathrm{H} & -5.11244300 & -2.20351100 & -2.67711800 \\ \mathrm{H} & -3.71907900 & -2.91836000 & -3.50698100 \\ \mathrm{C} & -3.36685200 & -0.19330800 & -3.49275500 \\ \mathrm{H} & -2.99288300 & -0.66592700 & -4.40840200 \\ \mathrm{H} & -4.41376200 & 0.07665200 & -3.67184800 \\ \mathrm{H} & -2.80329000 & 0.73122800 & -3.33704600 \\ \mathrm{H} & -6.24284900 & 1.11711300 & 0.44485700 \\ \mathrm{~N} & 2.50509200 & 3.05672000 & 0.38977900 \\ \mathrm{O} & 3.24946600 & 4.02865700 & 0.31472100 \\ \mathrm{C} & -0.59951900 & 3.41477800 & 1.02038300 \\ \mathrm{H} & -1.54953700 & 3.97020700 & 1.06861600 \\ \mathrm{H} & -0.27204100 & 3.23447500 & 2.04820100 \\ \mathrm{H} & -1.86732900 & 3.52057900 & -1.90169800 \\ \mathrm{C} & -1.12353100 & 3.47500800 & -2.96623700 \\ \mathrm{H} & -.16118800 & 3.88040100 & -1.81464600 \\ \mathrm{H} & & & \\ \mathrm{H} & & & \\ & & & \end{array}$

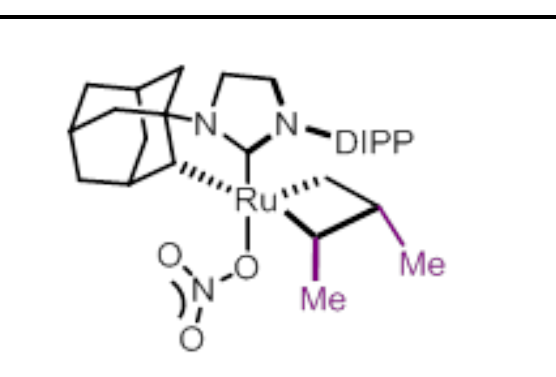

$26 c$

SCF Energy (M06, n-octanol): SCF Energy (B3LYP, gas-phase): Enthalpy Correction:

Free-Energy Correction:

\author{
$-1654.326015$ \\ $-1653.960799$ \\ 0.754060 \\ 0.653245
}

$\begin{array}{lrrr}\text { Ru } & 0.70396800 & 0.86762100 & -0.25366800 \\ \mathrm{O} & 2.04019000 & 2.38957300 & 1.52627100 \\ \mathrm{O} & 2.00105400 & 2.57534700 & -0.64999400 \\ \mathrm{~N} & 0.52404400 & -1.99119000 & 0.09688300 \\ \mathrm{~N} & -1.58035300 & -1.40228400 & -0.01758800 \\ \mathrm{C} & -0.29779900 & -0.91439000 & 0.00740800 \\ \mathrm{C} & -0.15581100 & -3.27243900 & -0.09436800 \\ \mathrm{H} & 0.20484100 & -4.03299300 & 0.60329500 \\ \mathrm{H} & -0.00497200 & -3.64404000 & -1.11797700 \\ \mathrm{C} & -1.61140800 & -2.87336400 & 0.16175700 \\ \mathrm{H} & -2.31478300 & -3.33524000 & -0.53506500 \\ \mathrm{H} & -1.92993700 & -3.11786700 & 1.18310400 \\ \mathrm{C} & 1.96792400 & -1.78327400 & -0.01753400 \\ \mathrm{C} & 2.44171000 & -1.87986700 & -1.49358200 \\ \mathrm{H} & 1.85554100 & -1.18724100 & -2.11032100 \\ \mathrm{H} & 2.25731300 & -2.89481400 & -1.87478200\end{array}$

S93 


$$
\begin{aligned}
& 3.94303900-1.54141500-1.58645300 \\
& 4.26320900-1.61240900-2.63434300 \\
& 4.74246200 \quad-2.54477700 \quad-0.73016000 \\
& 4.60672800-3.56603100-1.11414400 \\
& 5.81583600-2.32071100 \quad-0.79176100 \\
& 4.27264300 \quad-2.45844800 \quad 0.73692700 \\
& \begin{array}{lll}
4.83737400 & -3.17396600 & 1.34910500
\end{array} \\
& 2.76987300 \quad-2.80791000 \quad 0.81960100 \\
& 2.42365500 \quad-2.78198000 \quad 1.86134900 \\
& 2.60659100 \quad-3.82857600 \quad 0.44242000 \\
& 4.50240600 \quad-1.02615000 \quad 1.25941100 \\
& 4.19945000-0.94949700 \quad 2.31220400 \\
& 5.57251600-0.78000500 \quad 1.21594100 \\
& 3.69772900 \quad-0.01165800 \quad 0.40177300 \\
& \begin{array}{rrr}
3.88008000 & 0.99037500 & 0.79295200
\end{array} \\
& \begin{array}{lll}
2.20911500 & -0.36764700 & 0.54008600
\end{array} \\
& \begin{array}{lll}
1.92419900 & -0.34975200 & 1.60243400
\end{array} \\
& 4.16937100 \quad-0.10851200-1.06317900 \\
& 3.62832000 \quad 0.61958400 \quad-1.67920200 \\
& 5.23548700 \quad 0.14832100 \quad-1.12810700 \\
& \begin{array}{lll}
-2.80250300 & -0.69138800 & 0.25327600
\end{array} \\
& -3.72498800 \quad-0.49233600 \quad-0.80109400 \\
& \begin{array}{lll}
-4.92423500 & 0.17215400 & -0.51257000
\end{array} \\
& \begin{array}{lll}
-5.64354500 & 0.34411000 & -1.30799400
\end{array} \\
& -5.21739900 \\
& -4.32270500 \\
& -4.57371100 \\
& -3.10904100 \\
& -3.49313200 \\
& -2.45142300 \\
& -2.20461100 \\
& -1.26943700 \\
& -0.05604700 \\
& -0.85410100 \\
& -1.00386500 \\
& -0.61776000 \\
& 0.63538800 \\
& -2.01635900 \\
& -1.67915700 \\
& -6.15186500 \\
& -2.86380000 \\
& -3.78553000 \\
& -3.12503000 \\
& -2.18492400 \\
& -1.82770100 \\
& -2.70895800 \\
& -1.15869600 \\
& -1.30934000 \\
& -4.39675300 \\
& -5.45556100 \\
& -4.17634700 \\
& -4.26141800 \\
& -3.71011200 \\
& -3.06510500 \\
& -3.48335100 \\
& -4.74819800 \\
& 2.43596600 \\
& \text { 3. } 19334300 \\
& -0.65462400 \\
& -1.60449500 \\
& -0.36921200 \\
& 0.10262700 \\
& -0.83420900
\end{aligned}
$$




$\begin{array}{lrrr}\mathrm{H} & -1.06188700 & 3.44712400 & -3.05998200 \\ \mathrm{H} & -1.50925200 & 4.24254900 & -1.54035000 \\ \mathrm{H} & 0.19366800 & 3.86168700 & -1.87964700\end{array}$

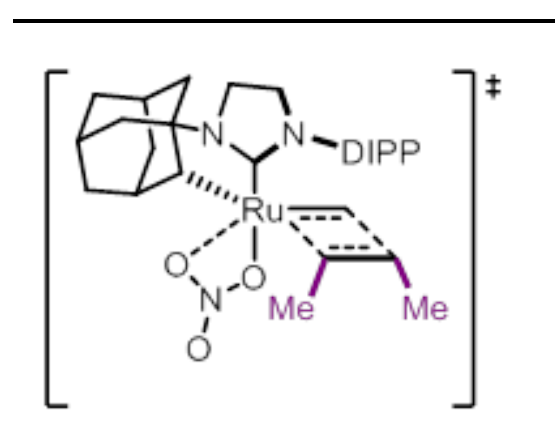

\section{7-TS}

SCF Energy (M06, n-octanol): $\quad-1654.307801$

SCF Energy (B3LYP, gas-phase): $\quad-1653.946927$

Enthalpy Correction:

0.751656

Free-Energy Correction:

0.651000

0.87519800

2.69855800

2.36623300

$-1.90270900$

$-1.46362100$

$-0.91042800$

$-3.22729800$

$-3.79818200$

$-3.80528400$

$-2.86110500$

$-3.49672800$

$-2.90121200$

$-1.66795700$

$-2.10817700$

$-1.62233100$

$-3.19498400$

$-1.72599500$

$-2.03211200$

$-2.45017700$

$-3.53948800$

$-2.19842300$

$-2.03031300$

$-2.54881000$

$-2.42263800$

$-2.15731000$

$-3.51254600$

$-0.50359200$

$-0.18231000$

$-0.23902700$

0.23113000

1.31052000

$-0.15395900$

0.03872900

$-0.19770000$

0.32086600

0.09380500

$-0.76742300$

$-0.81848900$

$-0.14725900$

$-0.16410700$

0.53142100

0.52418300

1.03058900

$-0.13276800$

$-1.64718600$

$-1.93524300$
$-0.36822200$

$-0.71347300$

1. 37804100

0.45906600

0.25083700

0.16914800

0.55749700

1.39909800

$-0.36170300$

0.73689900

0.15963100

1. 78991700

0.23611500

$-1.18934200$

$-1.92402700$

$-1.29799400$

$-1.44543700$

$-2.46203000$

$-0.41911800$

$-0.53811100$

$-0.59122000$

1.00670200

1. 74136300

1.25288300

2.27422900

1.14793900

1.14998200

2.17008100

0.97965900

0.12992100

0.23767700

0.45076000

1.51897500

$-1.29555800$

$-2.03386700$

$-1.49203800$

0.34802800

$-0.74583100$

$-0.62662200$

$-1.45511000$

0.54064500

1. 62651700

2.54371400

1.56360200

$-1.99746400$

$-1.96619700$ 
$-0.18649200$

$-0.69386600$ 0.48614700

$-0.29959900$

2.25603900

1. 67263500

1.92947700

1.16533700

1.07183000

3.07598600

4.05199000

$-0.88675600$

$-1.52591000$

$-0.59630200$

0.02154100

$-2.94055300$

$-3.57451500$

$-3.52375900$

$-2.70865700$

$-1.01214000$

$-0.52629900$

$-2.01648700$

$-1.11717700$

1.20799200

1.76459400

1.80337500

1.10666600

1.04959300

3.67478700

4.23208800

3.70298000

4.20782900

2.97974300

3.45868500

2.55202600

3. 76132800
2.81338800

2.55625600

$-2.18821600$

$-2.65421400$

$-0.32288900$

0.27814400

$-1.70356900$

$-1.99985400$

$-2.92356400$

0.51693200

0.81977200

$-3.31630000$

$-4.16703200$

$-3.44037500$

$-3.38111900$

$-1.98915800$

$-2.84583500$

$-1.07493600$

$-2.05251900$

3. 92103100

4.26485500

3.57283400

4.78653500

3.34264300

2. 61089200

3.60214900

4. 24905100

0.61087000

0.16145200

0.01926500

1. 22536700

$-0.38144700$

$-2.75173000$

$-2.56036900$

$-3.75822700$

$-2.73338300$ 


\section{Diasteromeric Pathway C}

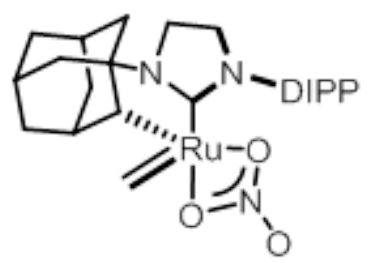

\section{9}

SCF Energy (M06, n-octanol): $\quad-1497.159821$

SCF Energy (B3LYP, gas-phase): $\quad-1496.729610$

Enthalpy Correction:

0.633925

Free-Energy Correction:

0.541623

\begin{abstract}
$-0.59213000$
0.23766000

$-1.30120900$

$-0.76711200$

1.37430500

0.14340700

$-0.19308100$

$-0.55656000$

$-0.43495300$

1.30973100

1.93105400

1. 66981700

$-2.17463900$

$-2.53034800$

$-1.80759000$

$-2.44508300$

$-3.95810800$

$-4.19807300$

$-4.95065900$

$-4.91703500$

$-5.97655400$

$-4.59815400$

$-5.30538300$

$-3.16708700$

$-2.90819600$

$-3.11842100$

$-4.68584800$

$-4.47011400$

$-5.70983600$

$-3.67937000$

$-3.74177000$

$-2.26931800$

$-2.11764900$

$-4.03366300$

$-3.34940500$

$-5.04472800$

2. 61362700

2. 95541700

4.18494400

4.46570800

5.05569100

4.71471400

5.40814000

3.49558800

2. 07756800

1.10349700

3. 18873200

2. 12797200

0.05592500

0.70051100
\end{abstract}

0.78999100

2.60608000

2.86397800

$-1.71220800$

$-1.20537900$

$-0.80170400$

$-2.64645800$

$-3.66595800$

$-2.34572300$

$-2.50363000$

$-2.48604500$

$-3.30757600$

$-1.29074000$

$-0.34713100$

0.48267800

$-0.88616300$

0.20498300

0.86448100

$-0.97490900$

$-1.52742400$

$-0.60029700$

$-1.91041200$

$-2.74959100$

$-2.46977900$

$-3.16000300$

$-3.03765000$

$-1.11145900$

$-1.76592800$

$-0.73505600$

0.06877100

0.62872400

$-0.52633000$

$-1.26175900$

0.99685700

1.85553100

1.40530600

$-0.48062300$

0.43623000

1.09881700

1.81311000

0.85538500

$-0.07051000$

$-0.26546400$

$-0.75812100$

0.67509200

0.21232100

$-1.80934100$

$-2.06863500$

0.31329300

$-0.53726600$
$-0.89605300$

$-0.02367900$

$-1.54242400$

0.49630400

0.45674300

0.05195700

1. 46783600

1. 31306000

2.49785200

1.16555800

2.06391300

0.51119000

0.42174500

1.60320300

1.64387700

2. 55781800

1. 42745700

2. 27164800

1.39155800

2. 34131200

1. 27643600

0.21558000

0.18680900

0.41170600

$-0.40195100$

1.35333400

$-1.10447200$

$-1.95969900$

$-1.23914800$

$-1.07488900$

$-2.01591400$

$-0.91924700$

$-1.71396900$

0.10539000

0.13091600

$-0.02783800$

0.34814100

1. 37079900

1.27349000

2.04163600

0.21615800

$-0.76483000$

$-1.57756700$

$-0.72018600$

2.59736900

2.41095900

$-1.78244400$

$-1.69897200$

$-2.52288100$

$-2.75917000$ 


$\begin{array}{rrrr}\mathrm{C} & 2.69053000 & -0.00632200 & 3.84066700 \\ \mathrm{H} & 3.65989900 & 0.43982300 & 4.09244200 \\ \mathrm{H} & 2.03121100 & 0.11407800 & 4.70866300 \\ \mathrm{H} & 2.85479600 & -1.07911500 & 3.68448800 \\ \mathrm{C} & 1.82400000 & 2.16865100 & 2.87703300 \\ \mathrm{H} & 1.12975600 & 2.27405500 & 3.71981000 \\ \mathrm{H} & 2.74763100 & 2.69112500 & 3.15326000 \\ \mathrm{H} & 1.39076100 & 2.66834300 & 2.00717100 \\ \mathrm{C} & 4.00657200 & -3.09540000 & -1.53529400 \\ \mathrm{H} & 3.74627100 & -3.86596000 & -2.27099000 \\ \mathrm{H} & 5.08144400 & -2.89705500 & -1.62238400 \\ \mathrm{H} & 3.82825200 & -3.50533900 & -0.53484700 \\ \mathrm{C} & 3.42997400 & -1.29708200 & -3.21550200 \\ \mathrm{H} & 4.49517600 & -1.13035200 & -3.41213100 \\ \mathrm{H} & 3.07727000 & -2.03578200 & -3.94496900 \\ \mathrm{H} & 2.90520200 & -0.35451600 & -3.39991500 \\ \mathrm{H} & 6.00466000 & 1.38224200 & 0.16075700 \\ \mathrm{~N} & -0.49934600 & 3.43094900 & -0.72583200 \\ \mathrm{O} & -0.41239800 & 4.63021900 & -0.58618100 \\ \mathrm{H} & -0.18818900 & 0.97271900 & -3.36921300\end{array}$

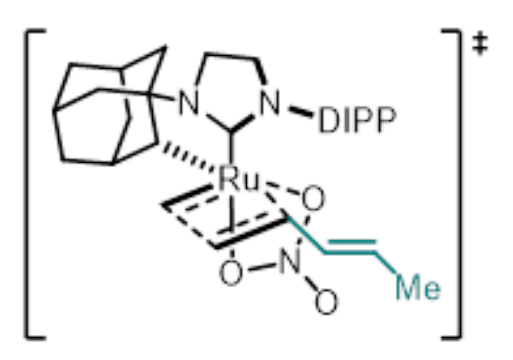

40-TS

SCF Energy (M06, n-octanol):

SCF Energy (B3LYP, gas-phase):

Enthalpy Correction:

$-1692.365370$

Free-Energy Correction:

$-1692.027744$

0.757100

0.654337

$$
\begin{array}{rr}
0.52168200 & -0.89645800 \\
1.87149800 & 0.56117300 \\
2.16737000 & -1.58714300 \\
-2.06308000 & 0.40552900 \\
-1.44224700 & 0.40724200 \\
-1.05120600 & 0.04245100 \\
-3.08385000 & 1.23209600 \\
-4.09020300 & 0.96098500 \\
-2.92333800 & 2.29743300 \\
-2.83723400 & 0.90332700 \\
-2.93871000 & 1.76299300 \\
-3.51429100 & 0.11487400 \\
-1.86466300 & 0.35026800 \\
-1.34829400 & 1.71018900 \\
-0.42362100 & 1.97729900 \\
-2.08925500 & 2.49755400 \\
-1.08609200 & 1.61401100 \\
-0.73886600 & 2.58923100 \\
-2.38434300 & 1.21669100 \\
-3.16185900 & 1.97947500 \\
-2.20250900 & 1.15858900 \\
-2.86849300 & -0.14774000 \\
-3.78860700 & -0.44238900 \\
-3.17270400 & -0.01245900 \\
-3.57257500 & -0.95242200 \\
-3.94216400 & 0.76116300 \\
-1.77150000 & -1.21006200 \\
-2.11947800 & -2.19660100 \\
-1.57198200 & -1.29151100 \\
-0.47357700 & -0.81193300
\end{array}
$$




\begin{tabular}{|c|c|c|}
\hline-3.88833900 & 0.29541000 & -1.57312300 \\
\hline-2.21329600 & -0.83976200 & -0.77234400 \\
\hline-2.01259800 & -1.36981900 & -1.71116400 \\
\hline-4.27840700 & -0.00194700 & 0.54779000 \\
\hline-3.82126300 & 0.93663700 & 0.86445700 \\
\hline-5.35781500 & 0.18018700 & 0.44784200 \\
\hline 2.80382100 & -0.69746800 & 0.41212000 \\
\hline 3.06739200 & 0.19217800 & 1.48602800 \\
\hline 4.30679300 & 0.84384100 & 1.51159800 \\
\hline 4.52757500 & 1.53830800 & 2.31562600 \\
\hline 5.26904100 & 0.61285900 & 0.53223700 \\
\hline 5.00728600 & -0.28447300 & -0.49694100 \\
\hline 5.76805200 & -0.46948100 & -1.24984800 \\
\hline 3.78002800 & -0.95605200 & -0.57981900 \\
\hline 2.07559800 & 0.40782000 & 2.63166600 \\
\hline 1.06873700 & 0.22520400 & 2.24435500 \\
\hline 3.55947900 & -1.96024400 & -1.71025000 \\
\hline 2.50187700 & -2.24378000 & -1.70492800 \\
\hline 1.01107400 & 2.34502900 & -0.56456700 \\
\hline 1.36236900 & 2.02461600 & 0.41007800 \\
\hline 0.22238700 & 0.09094100 & -2.54544300 \\
\hline 1.01816200 & -0.62285200 & -2.78612100 \\
\hline 1.51479200 & 1.68328000 & -1.69002000 \\
\hline 2.32787800 & 0.98145600 & -1.56908900 \\
\hline 4.39851200 & -3.23682300 & -1.48499000 \\
\hline 4.18825500 & -3.97830500 & -2.26495000 \\
\hline 5.47081900 & -3.01024100 & -1.51887600 \\
\hline 4.19079300 & -3.69667600 & -0.51291800 \\
\hline 3.86807600 & -1.37330000 & -3.10198300 \\
\hline 4.93024100 & -1.12827400 & -3.21347900 \\
\hline 3.61786400 & -2.10308200 & -3.88079200 \\
\hline 3.29315400 & -0.46248400 & -3.29765600 \\
\hline 2.33071200 & -0.59724500 & 3.77682000 \\
\hline 2.24877600 & -1.63618800 & 3.44368200 \\
\hline 3.33528400 & -0.46073200 & 4.19498700 \\
\hline 1.60512600 & -0.44648700 & 4.58486400 \\
\hline 2.09240500 & 1.83708400 & 3.20667400 \\
\hline 1.24545400 & 1.96822700 & 3.88885400 \\
\hline 3.00446900 & 2.03673100 & 3.78147900 \\
\hline 2.01618900 & 2.60023100 & 2.42595200 \\
\hline 6.22496700 & 1.12786900 & 0.57674800 \\
\hline 1.45235800 & 2.19086900 & -2.64980500 \\
\hline-2.29254700 & 2.52114400 & -0.41142200 \\
\hline-3.11147900 & 3.40832300 & -0.23313600 \\
\hline 0.40865300 & 3.66910900 & -0.65103200 \\
\hline 0.21400800 & 4.05325800 & -1.65200000 \\
\hline 0.08098600 & 4.42038600 & 0.41446200 \\
\hline 0.24602700 & 4.01338500 & 1.41226000 \\
\hline-0.55887700 & 5.77247800 & 0.34484100 \\
\hline 0.00069000 & 6.51507800 & 0.92975300 \\
\hline-0.63504500 & 6.13014400 & -0.68725400 \\
\hline-1.57423100 & 5.73076800 & 0.76121900 \\
\hline-0.16158000 & 0.60082600 & -3.44135700 \\
\hline
\end{tabular}

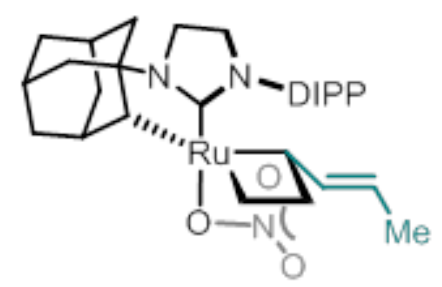

41

SCF Energy (M06, n-octanol): $\quad$-1692.399534

SCF Energy (B3LYP, gas-phase): $\quad-1692.055053$

Enthalpy Correction:

0.759883

Free-Energy Correction:

0.654916 


\begin{tabular}{|c|c|c|c|}
\hline $\mathrm{Ru}$ & -0.61833900 & 0.34531500 & -0.81261100 \\
\hline 0 & -1.75546900 & -0.71983300 & -3.07023900 \\
\hline 0 & -1.96772500 & 1.29690600 & -2.24980600 \\
\hline $\mathrm{N}$ & -0.26279500 & -1.26677300 & 1.55376800 \\
\hline $\mathrm{N}$ & 1.78205300 & -0.64685000 & 1.07615700 \\
\hline $\mathrm{C}$ & 0.48186900 & -0.58493400 & 0.64839700 \\
\hline $\mathrm{C}$ & 0.46972600 & -1.62932000 & 2.76730900 \\
\hline $\mathrm{H}$ & 0.22949400 & -2.64279300 & 3.10012800 \\
\hline $\mathrm{H}$ & 0.23538900 & -0.93422800 & 3.58598200 \\
\hline $\mathrm{C}$ & 1.92011400 & -1.48678600 & 2.29003800 \\
\hline $\mathrm{H}$ & 2.57021900 & -1.00419400 & 3.02392000 \\
\hline $\mathrm{H}$ & 2.36170300 & -2.45587900 & 2.02552000 \\
\hline $\mathrm{C}$ & -1.72050300 & -1.22822800 & 1.42058600 \\
\hline $\mathrm{C}$ & -2.32567300 & -0.02195500 & 2.18740600 \\
\hline $\mathrm{H}$ & -1.83602300 & 0.90340800 & 1.85863200 \\
\hline $\mathrm{H}$ & -2.13168600 & -0.13124500 & 3.26442900 \\
\hline $\mathrm{C}$ & -3.84287000 & 0.05861800 & 1.92471500 \\
\hline $\mathrm{H}$ & -4.25792800 & 0.91275300 & 2.47576800 \\
\hline $\mathrm{C}$ & -4.51050000 & -1.24540700 & 2.40670200 \\
\hline $\mathrm{H}$ & -4.36404100 & -1.36977000 & 3.48933900 \\
\hline $\mathrm{H}$ & -5.59417300 & -1.20023000 & 2.23431800 \\
\hline $\mathrm{C}$ & -3.90857200 & -2.44463900 & 1.64486000 \\
\hline $\mathrm{H}$ & -4.38235500 & -3.37468800 & 1.98538900 \\
\hline $\mathrm{C}$ & -2.39080100 & -2.52528700 & 1.92736700 \\
\hline $\mathrm{H}$ & -1.94785000 & -3.39091300 & 1.41706900 \\
\hline $\mathrm{H}$ & -2.22151000 & -2.65602000 & 3.00676500 \\
\hline $\mathrm{C}$ & -4.15244600 & -2.25756100 & 0.13277000 \\
\hline $\mathrm{H}$ & -3.75694300 & -3.11523700 & -0.42741100 \\
\hline $\mathrm{H}$ & -5.23227700 & -2.21545000 & -0.06694700 \\
\hline $\mathrm{C}$ & -3.47710200 & -0.94794500 & -0.35762000 \\
\hline $\mathrm{H}$ & -3.65598000 & -0.83933300 & -1.42928700 \\
\hline $\mathrm{C}$ & -1.97010500 & -1.08408300 & -0.09253200 \\
\hline $\mathrm{H}$ & -1.58420800 & -1.97661000 & -0.60699800 \\
\hline $\mathrm{C}$ & -4.07996100 & 0.24532300 & 0.41221100 \\
\hline $\mathrm{H}$ & -3.62985100 & 1.18329100 & 0.06559600 \\
\hline $\mathrm{H}$ & -5.15719500 & 0.31330900 & 0.20771200 \\
\hline $\mathrm{C}$ & 2.96717000 & -0.42013400 & 0.29049700 \\
\hline $\mathrm{C}$ & 3.76974100 & 0.70906300 & 0.57433600 \\
\hline $\mathrm{C}$ & 4.93166600 & 0.90116900 & -0.18463000 \\
\hline $\mathrm{H}$ & 5.55894700 & 1.76620700 & 0.00995100 \\
\hline $\mathrm{C}$ & 5.30186700 & -0.00196400 & -1.17649400 \\
\hline $\mathrm{C}$ & 4.52753500 & -1.13553300 & -1.40693200 \\
\hline $\mathrm{H}$ & 4.84026000 & -1.84979200 & -2.16323400 \\
\hline $\mathrm{C}$ & 3.35766400 & -1.37958700 & -0.67613000 \\
\hline $\mathrm{C}$ & 3.45335700 & 1.67037300 & 1.71825200 \\
\hline $\mathrm{H}$ & 2.43948100 & 1.44779600 & 2.06667700 \\
\hline $\mathrm{C}$ & 2.59773400 & -2.68700800 & -0.90718300 \\
\hline $\mathrm{H}$ & 1.68035900 & -2.66282900 & -0.31243000 \\
\hline $\mathrm{C}$ & -0.00282800 & 2.16289600 & -0.19391300 \\
\hline $\mathrm{H}$ & 0.65836000 & 2.18880400 & 0.67454400 \\
\hline $\mathrm{C}$ & 0.85392100 & 0.59750100 & -2.17018200 \\
\hline $\mathrm{H}$ & 1.79475600 & 0.07181200 & -2.04338000 \\
\hline $\mathrm{C}$ & 0.83685900 & 2.02648000 & -1.55991600 \\
\hline $\mathrm{H}$ & 1.86457500 & 2.29414300 & -1.30171000 \\
\hline $\mathrm{C}$ & 3.43935100 & -3.88829600 & -0.42241300 \\
\hline $\mathrm{H}$ & 2.86524900 & -4.81771400 & -0.51474300 \\
\hline $\mathrm{H}$ & 4.35148200 & -3.99880500 & -1.02042400 \\
\hline $\mathrm{H}$ & 3.74444000 & -3.77804900 & 0.62468800 \\
\hline $\mathrm{C}$ & 2.17082200 & -2.89610000 & -2.37326500 \\
\hline $\mathrm{H}$ & 3.03454600 & -2.93374200 & -3.04739900 \\
\hline $\mathrm{H}$ & 1.63688300 & -3.84840100 & -2.47111200 \\
\hline $\mathrm{H}$ & 1.50112000 & -2.10367200 & -2.71738600 \\
\hline
\end{tabular}




$\begin{array}{rrrr}\mathrm{C} & 4.42018400 & 1.43908300 & 2.90064300 \\ \mathrm{H} & 4.40680900 & 0.39799500 & 3.24230200 \\ \mathrm{H} & 5.45208600 & 1.67676200 & 2.61597300 \\ \mathrm{H} & 4.15036300 & 2.07954800 & 3.74883100 \\ \mathrm{C} & 3.48546800 & 3.15209000 & 1.29888500 \\ \mathrm{H} & 3.20124100 & 3.78736700 & 2.14586700 \\ \mathrm{H} & 4.48662700 & 3.46360600 & 0.98009700 \\ \mathrm{H} & 2.79211000 & 3.35830900 & 0.47799400 \\ \mathrm{H} & 6.20398600 & 0.16781800 & -1.75819200 \\ \mathrm{H} & 0.41620400 & 2.72936500 & -2.28044000 \\ \mathrm{~N} & -2.25608500 & 0.42779100 & -3.18256100 \\ \mathrm{O} & -2.99268000 & 0.76157300 & -4.10295000 \\ \mathrm{C} & -1.01296400 & 3.23795100 & -0.16320200 \\ \mathrm{H} & -1.64086100 & 3.34187500 & -1.04711300 \\ \mathrm{C} & -1.21360500 & 4.04543300 & 0.89240800 \\ \mathrm{H} & -0.57676900 & 3.92923200 & 1.77248400 \\ \mathrm{C} & -2.25810700 & 5.12094700 & 0.96704700 \\ \mathrm{H} & -2.94844200 & 4.95028000 & 1.80515600 \\ \mathrm{H} & -1.80487300 & 6.10824600 & 1.13360800 \\ \mathrm{H} & -2.84942100 & 5.16909200 & 0.04668300 \\ \mathrm{H} & 0.47332100 & 0.54339200 & -3.19004000 \\ & & \end{array}$

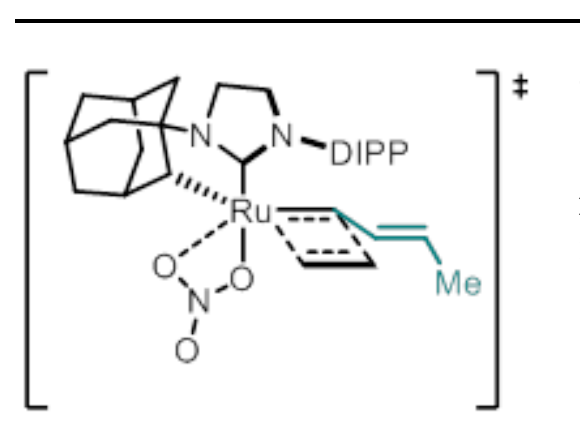

\section{2-TS}

SCF Energy (M06, n-octanol): SCF Energy (B3LYP, gas-phase): Enthalpy Correction: Free-Energy Correction:

\author{
$-1692.386772$ \\ $-1692.046883$ \\ 0.758093 \\ 0.653890
}

\begin{abstract}
0.56254300
1.88428900

1.25242600

0.31641500

$-1.74445100$

$-0.45331500$

$-0.39602000$

$-0.13904900$

$-0.15519500$

$-1.85538200$

$-2.49803700$

$-2.29237700$

1.77588700

2.36840800

1.86565000

2.18800400

3.88226600

4.29152700

4.56928400

4.42860700

5.65181100

3.97777400

4.46096700

2.46022500

2.02379800

2. 30101800

4.22573900

3. 84756100
\end{abstract}

$$
\begin{array}{r}
0.27158700 \\
0.89585300 \\
-1.17473300 \\
-1.11538300 \\
-0.55152200 \\
-0.51699400 \\
-1.40856700 \\
-2.39720400 \\
-0.66279500 \\
-1.31041900 \\
-0.78714800 \\
-2.29844200 \\
-1.06819000 \\
0.15935600 \\
1.06884600 \\
0.06974000 \\
0.25025000 \\
1.12651700 \\
-1.02909700 \\
-1.12109900 \\
-0.97642400 \\
-2.25750600 \\
-3.17149500 \\
-2.34268100 \\
-3.22841600 \\
-2.44311800 \\
-2.11417300 \\
-2.99540800
\end{array}
$$

0.88669100

2.54854400

2.77189700

$-1.64321600$

$-1.16142000$

$-0.69988200$

$-2.88734700$

$-3.27760000$

$-3.65802400$

$-2.42832200$

$-3.14075000$

$-2.23457600$

$-1.50474200$

$-2.24198600$

$-1.89637500$

$-3.32346300$

$-1.96621800$

$-2.48685300$

$-2.48492500$

$-3.57168800$

$-2.30628100$

$-1.76251900$

$-2.13267100$

$-2.05436200$

$-1.57407900$

$-3.13888500$

$-0.24500200$

0.28968700

\section{S101}




\begin{tabular}{|c|c|c|c|}
\hline $\mathrm{H}$ & 5.30699700 & -2.06220100 & -0.05286400 \\
\hline $\mathrm{C}$ & 3.52403400 & -0.83519600 & 0.28437000 \\
\hline $\mathrm{H}$ & 3.71088600 & -0.74392100 & 1.35869300 \\
\hline C & 2.01741000 & -0.99074100 & 0.01411200 \\
\hline $\mathrm{H}$ & 1.69155800 & -1.95047500 & 0.44176300 \\
\hline C & 4.10748800 & 0.39145500 & -0.44655100 \\
\hline $\mathrm{H}$ & 3.63549200 & 1.30982300 & -0.07798400 \\
\hline $\mathrm{H}$ & 5.18277400 & 0.47500200 & -0.23547300 \\
\hline C & -2.94586500 & -0.35936400 & -0.39516300 \\
\hline C & -3.72872200 & 0.79429200 & -0.63383900 \\
\hline C & -4.90756200 & 0.95774600 & 0.10500500 \\
\hline $\mathrm{H}$ & -5.52059300 & 1.83992500 & -0.05588500 \\
\hline C & -5.31350500 & 0.00323900 & 1.03306500 \\
\hline C & -4.55815600 & -1.15151200 & 1.21694200 \\
\hline $\mathrm{H}$ & -4.89838200 & -1.90442700 & 1.92220200 \\
\hline C & -3.37168700 & -1.36770500 & 0.50390100 \\
\hline C & -3.37344500 & 1.80922200 & -1.71918600 \\
\hline $\mathrm{H}$ & -2.35667900 & 1.58668400 & -2.05911900 \\
\hline $\mathrm{C}$ & -2.62945800 & -2.69371800 & 0.67930400 \\
\hline $\mathrm{H}$ & -1.70149000 & -2.64824800 & 0.10259600 \\
\hline $\mathrm{C}$ & 0.37029400 & 2.03983300 & 0.15583200 \\
\hline $\mathrm{H}$ & -0.31062000 & 2.27908200 & -0.66980500 \\
\hline C & -1.05610500 & 0.58762000 & 2.28502700 \\
\hline $\mathrm{H}$ & -1.93491000 & 0.00338100 & 2.04417300 \\
\hline C & -0.94215600 & 1.90158700 & 1.73224600 \\
\hline $\mathrm{H}$ & -1.76584700 & 2.25543900 & 1.12095800 \\
\hline $\mathrm{N}$ & 1.94484000 & -0.20893300 & 3.22472600 \\
\hline 0 & 2.63482500 & -0.29378000 & 4.22921700 \\
\hline $\mathrm{C}$ & -3.38911000 & 3.26909500 & -1.22883000 \\
\hline $\mathrm{H}$ & -3.09840300 & 3.94116200 & -2.04481700 \\
\hline $\mathrm{H}$ & -4.38575900 & 3.57674300 & -0.89279100 \\
\hline $\mathrm{H}$ & -2.69278900 & 3.42998200 & -0.39987900 \\
\hline C & -4.32147900 & 1.65546500 & -2.92917100 \\
\hline $\mathrm{H}$ & -4.02172900 & 2.32838800 & -3.74147100 \\
\hline $\mathrm{H}$ & -4.32354200 & 0.63133500 & -3.31843100 \\
\hline $\mathrm{H}$ & -5.35332500 & 1.90252200 & -2.65235300 \\
\hline C & -3.47337800 & -3.85794900 & 0.11523600 \\
\hline $\mathrm{H}$ & -4.39893400 & -3.99143600 & 0.68761000 \\
\hline $\mathrm{H}$ & -3.75532500 & -3.68829600 & -0.93052600 \\
\hline $\mathrm{H}$ & -2.90994400 & -4.79682900 & 0.16871100 \\
\hline C & -2.22913200 & -2.98262100 & 2.13949200 \\
\hline $\mathrm{H}$ & -1.54004000 & -2.22830900 & 2.52838700 \\
\hline $\mathrm{H}$ & -3.10317500 & -3.02826100 & 2.79996900 \\
\hline $\mathrm{H}$ & -1.72219000 & -3.95264200 & 2.19829100 \\
\hline $\mathrm{H}$ & -6.22864900 & 0.15039700 & 1.60041600 \\
\hline $\mathrm{H}$ & -0.44855300 & 2.65899000 & 2.33348500 \\
\hline $\mathrm{H}$ & -0.64057900 & 0.41392200 & 3.27346200 \\
\hline $\mathrm{C}$ & 1.29958500 & 3.12574900 & 0.46175100 \\
\hline $\mathrm{H}$ & 1.97387700 & 2.98207800 & 1.30531000 \\
\hline C & 1.39046000 & 4.24586500 & -0.28221500 \\
\hline $\mathrm{H}$ & 0.70447500 & 4.37313400 & -1.12229300 \\
\hline C & 2.38476800 & 5.34638300 & -0.06397900 \\
\hline $\mathrm{H}$ & 3.03131500 & 5.47661300 & -0.94301100 \\
\hline $\mathrm{H}$ & 1.87961500 & 6.30912000 & 0.09561100 \\
\hline $\mathrm{H}$ & 3.02347300 & 5.14574500 & 0.80203100 \\
\hline
\end{tabular}

\section{S102}




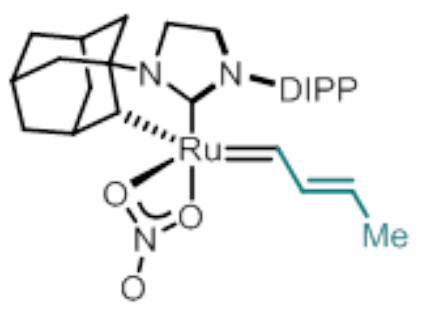

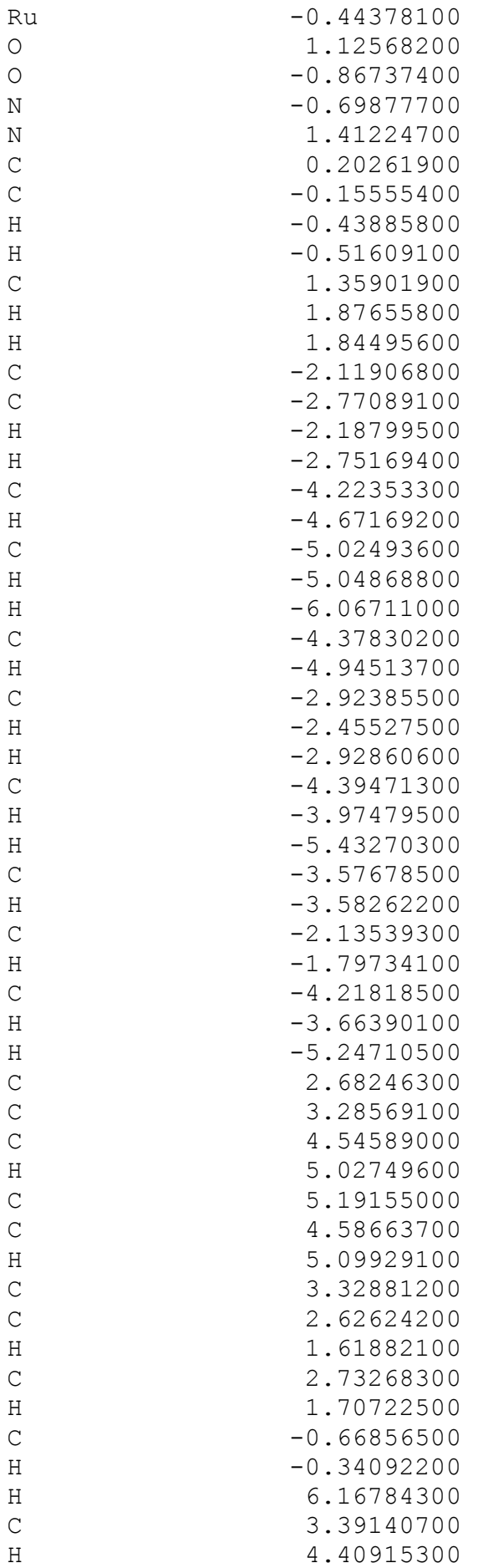

43

SCF Energy (M06, n-octanol): $\quad-1613.834302$

SCF Energy (B3LYP, gas-phase): $\quad-1613.466877$

Enthalpy Correction:

0.700216

Free-Energy Correction:

$$
\begin{aligned}
& 0.48414000 \\
& 1.02448200 \\
& 1.84131100 \\
& -1.74436900 \\
& -1.13324500 \\
& -0.86177400 \\
& -2.55380300 \\
& -3.60634800 \\
& -2.18797500 \\
& -2.32918100 \\
& -2.14606100 \\
& -3.17727800 \\
& -1.50089500 \\
& -0.69511400 \\
& 0.21377800 \\
& -1.28963200 \\
& -0.33418000 \\
& 0.23573200 \\
& -1.63109700 \\
& -2.23115700 \\
& -1.39275100 \\
& -2.43600800 \\
& -3.36118500 \\
& -2.80445700 \\
& -3.40421600 \\
& -3.41554000 \\
& -1.57750800 \\
& -2.14357300 \\
& -1.33259900 \\
& -0.27660000 \\
& 0.32419100 \\
& -0.68867800 \\
& -1.38269100 \\
& 0.52160100 \\
& 1.45191400 \\
& 0.79949300 \\
& -0.60395600 \\
& 0.41275400 \\
& 0.87981700 \\
& 1.66416300 \\
& 0.35679700 \\
& -0.65269200 \\
& -1.05645000 \\
& -1.15835600 \\
& 0.99203900 \\
& 0.56923400 \\
& -2.30119400 \\
& -2.46850700 \\
& \text { 1. } 76408400 \\
& 1.60612500 \\
& 0.73530000 \\
& 0.58413400 \\
& 0.99201200
\end{aligned}
$$

\section{S103}




$\begin{array}{rrrr}\mathrm{H} & 2.88009900 & 0.96156800 & 4.76937100 \\ \mathrm{H} & 3.47405800 & -0.50543600 & 3.96559400 \\ \mathrm{C} & 2.47761100 & 2.52314500 & 2.51899900 \\ \mathrm{H} & 1.93436200 & 2.89778600 & 3.39536600 \\ \mathrm{H} & 3.45263200 & 3.02384300 & 2.49961600 \\ \mathrm{H} & 1.92889700 & 2.82143600 & 1.62024800 \\ \mathrm{C} & 3.52095100 & -3.60943000 & -1.14392000 \\ \mathrm{H} & 3.56615400 & -3.88519800 & -0.08376600 \\ \mathrm{H} & 3.05431000 & -4.43684700 & -1.69190800 \\ \mathrm{H} & 4.55282300 & -3.51243100 & -1.50153600 \\ \mathrm{C} & 2.65363300 & -1.97166700 & -2.87114600 \\ \mathrm{H} & 3.65160100 & -1.87777300 & -3.31527800 \\ \mathrm{H} & 2.13472200 & -2.77837600 & -3.40337600 \\ \mathrm{H} & 2.11949300 & -1.03350300 & -3.04337800 \\ \mathrm{~N} & 0.34189300 & 1.76314300 & -3.07057900 \\ \mathrm{O} & 0.73972700 & 2.32130700 & -4.07028200 \\ \mathrm{C} & -1.23668800 & 3.07582400 & 0.20318600 \\ \mathrm{H} & -1.55931900 & 3.31125500 & -0.80997700 \\ \mathrm{C} & -1.37149100 & 4.00755100 & 1.17455000 \\ \mathrm{H} & -1.03251500 & 3.76147400 & 2.18276800 \\ \mathrm{C} & -1.95235500 & 5.37483600 & 0.98450000 \\ \mathrm{H} & -2.82443600 & 5.52855400 & 1.63567300 \\ \mathrm{H} & -1.22441200 & 6.15317300 & 1.25349600 \\ \mathrm{H} & -2.26409500 & 5.54089800 & -0.05149700 \\ & & & \end{array}$

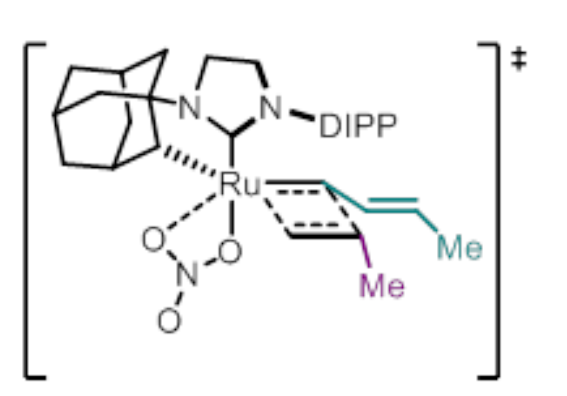

\title{
44-TS
}

SCF Energy (M06, n-octanol): $\quad-1731.679930$

SCF Energy (B3LYP, gas-phase):

Enthalpy Correction:

Free-Energy Correction:

\author{
$-1731.359476$ \\ 0.788290 \\ 0.682205
}

$$
\begin{array}{r}
0.57330000 \\
1.88740800 \\
1.30852200 \\
0.35387200 \\
-1.71680500 \\
-0.42916700 \\
-0.34199500 \\
-0.07321300 \\
-0.09855700 \\
-1.80652600 \\
-2.44557000 \\
-2.23666100 \\
1.81046300 \\
2.40915300 \\
1.89835900 \\
2.24046300 \\
3.91921000 \\
4.33299600 \\
4.61604800 \\
4.48915100 \\
5.69597700 \\
4.01879600 \\
4.50931300 \\
2.50537200 \\
2.06608300
\end{array}
$$

$$
\begin{array}{r}
0.42213000 \\
1.47717100 \\
-0.47109800 \\
-1.55741000 \\
-0.91360700 \\
-0.75420900 \\
-2.14669800 \\
-3.19721000 \\
-1.60344000 \\
-1.95681800 \\
-1.62775700 \\
-2.87410200 \\
-1.46995600 \\
-0.45256800 \\
0.51033800 \\
-0.80057200 \\
-0.29010400 \\
0.43599700 \\
-1.65338900 \\
-2.00719600 \\
-1.55261200 \\
-2.67330300 \\
-3.64701000 \\
-2.83558500 \\
-3.58096300
\end{array}
$$

0.73595000

2.17796900

2.95753300

$-1.36734600$

$-1.05472500$

$-0.60520900$

$-2.51182800$

$-2.65260800$

$-3.43582100$

$-2.10270300$

$-2.92594600$

$-1.68054200$

$-1.22546800$

$-2.22951700$

$-2.11829500$

$-3.25945900$

$-1.96728500$

$-2.67989600$

$-2.15217400$

$-3.18553700$

$-1.97901100$

$-1.16044400$

$-1.29161400$

$-1.44022700$

$-0.76405900$

\section{S104}




\begin{tabular}{|c|c|c|c|}
\hline $\mathrm{H}$ & 2.35948100 & -3.19703500 & -2.46962000 \\
\hline $\mathrm{C}$ & 4.24414000 & -2.16477800 & 0.27986400 \\
\hline $\mathrm{H}$ & 3.86011900 & -2.89219700 & 1.00719200 \\
\hline $\mathrm{H}$ & 5.32215900 & -2.06113300 & 0.46907400 \\
\hline $\mathrm{C}$ & 3.53357000 & -0.79897300 & 0.47470500 \\
\hline $\mathrm{H}$ & 3.70771100 & -0.45294800 & 1.49768100 \\
\hline $\mathrm{C}$ & 2.03069900 & -1.02200500 & 0.23206000 \\
\hline $\mathrm{H}$ & 1.69562000 & -1.84411700 & 0.88137000 \\
\hline C & 4.12586700 & 0.21598900 & -0.52435800 \\
\hline $\mathrm{H}$ & 3.65162200 & 1.19584300 & -0.39401800 \\
\hline $\mathrm{H}$ & 5.19867000 & 0.34988900 & -0.32719600 \\
\hline C & -2.92552800 & -0.58811900 & -0.34621300 \\
\hline $\mathrm{C}$ & -3.74525500 & 0.45607800 & -0.83492200 \\
\hline C & -4.92721400 & 0.74708400 & -0.14130200 \\
\hline $\mathrm{H}$ & -5.57004800 & 1.54926600 & -0.49268900 \\
\hline C & -5.29905800 & 0.01942400 & 0.98537500 \\
\hline C & -4.50673500 & -1.03888300 & 1.42110600 \\
\hline $\mathrm{H}$ & -4.82008400 & -1.61873200 & 2.28477200 \\
\hline $\mathrm{C}$ & -3.31729100 & -1.37721300 & 0.76292000 \\
\hline $\mathrm{C}$ & -3.42486100 & 1.20617100 & -2.12687200 \\
\hline $\mathrm{H}$ & -2.39907200 & 0.95008900 & -2.41184200 \\
\hline C & -2.53436200 & -2.60604100 & 1.23091700 \\
\hline $\mathrm{H}$ & -1.61763700 & -2.67799900 & 0.63918100 \\
\hline C & 0.29553400 & 1.96476700 & -0.38236200 \\
\hline $\mathrm{H}$ & -0.40043200 & 1.97253200 & -1.22893400 \\
\hline $\mathrm{C}$ & -1.05286800 & 0.99777100 & 1.98447000 \\
\hline $\mathrm{H}$ & -1.90670500 & 0.34118300 & 1.87644000 \\
\hline $\mathrm{C}$ & -1.01556300 & 2.19161300 & 1.16860500 \\
\hline $\mathrm{H}$ & -1.84619500 & 2.26074300 & 0.47046900 \\
\hline C & -0.63005700 & 3.50081400 & 1.83484700 \\
\hline $\mathrm{H}$ & -0.45595600 & 4.30182400 & 1.11110000 \\
\hline $\mathrm{H}$ & -1.44919600 & 3.81271600 & 2.49604200 \\
\hline $\mathrm{H}$ & 0.26982600 & 3.37588800 & 2.44291900 \\
\hline $\mathrm{N}$ & 1.96981400 & 0.59826500 & 3.12961200 \\
\hline 0 & 2.65445400 & 0.81236000 & 4.11940600 \\
\hline $\mathrm{C}$ & -3.49848800 & 2.73841200 & -1.98827300 \\
\hline $\mathrm{H}$ & -3.22090600 & 3.21290100 & -2.93681600 \\
\hline $\mathrm{H}$ & -4.50989400 & 3.07825600 & -1.73821200 \\
\hline $\mathrm{H}$ & -2.81970400 & 3.11333500 & -1.21594100 \\
\hline $\mathrm{C}$ & -4.36163500 & 0.74051600 & -3.26348800 \\
\hline $\mathrm{H}$ & -4.08621700 & 1.21990600 & -4.21051100 \\
\hline $\mathrm{H}$ & -4.32106200 & -0.34494000 & -3.40645900 \\
\hline $\mathrm{H}$ & -5.40346900 & 1.00347000 & -3.04486600 \\
\hline C & -3.35402500 & -3.89028300 & 0.97834400 \\
\hline $\mathrm{H}$ & -4.26438000 & -3.90778600 & 1.58904000 \\
\hline $\mathrm{H}$ & -3.66036200 & -3.97902200 & -0.07057500 \\
\hline $\mathrm{H}$ & -2.76238900 & -4.77611400 & 1.23719500 \\
\hline C & -2.10183000 & -2.52835200 & 2.70850500 \\
\hline $\mathrm{H}$ & -1.41881700 & -1.69377900 & 2.88754700 \\
\hline $\mathrm{H}$ & -2.96273000 & -2.42173000 & 3.37891800 \\
\hline $\mathrm{H}$ & -1.57793000 & -3.44920900 & 2.98972700 \\
\hline $\mathrm{H}$ & -6.21655200 & 0.26531600 & 1.51341900 \\
\hline $\mathrm{C}$ & 1.25244900 & 3.06875900 & -0.42172600 \\
\hline $\mathrm{H}$ & 1.95727800 & 3.15211900 & 0.40418200 \\
\hline $\mathrm{C}$ & 1.33850600 & 3.92966800 & -1.45551000 \\
\hline $\mathrm{H}$ & 0.62345100 & 3.83617600 & -2.27551900 \\
\hline $\mathrm{C}$ & 2.36618300 & 5.01353500 & -1.59017800 \\
\hline $\mathrm{H}$ & 2.98259900 & 4.86713800 & -2.48820100 \\
\hline $\mathrm{H}$ & 1.89133700 & 5.99881700 & -1.69664600 \\
\hline $\mathrm{H}$ & 3.03254400 & 5.04603600 & -0.72216100 \\
\hline $\mathrm{H}$ & -0.67863200 & 1.08596700 & 3.00292700 \\
\hline
\end{tabular}

\section{S105}




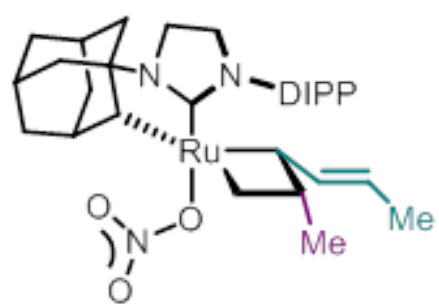

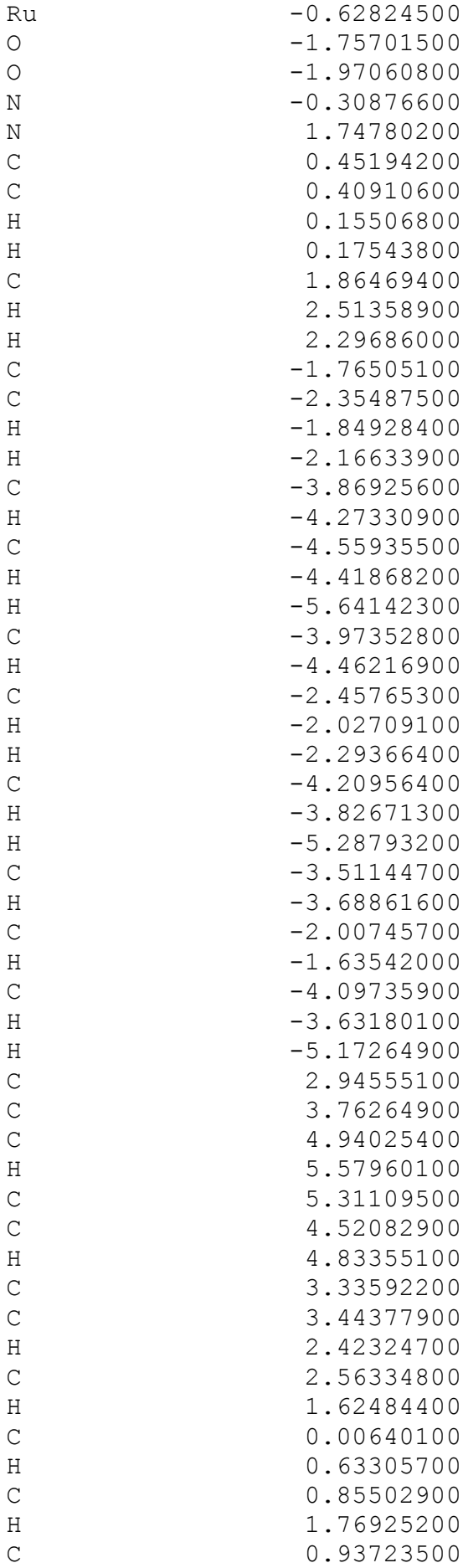

45

SCF Energy (M06, n-octanol): $\quad-1731.690010$

SCF Energy (B3LYP, gas-phase): $\quad-1731.366153$

Enthalpy Correction:

0.789787

Free-Energy Correction:
0.44574600
-0.07663900
1.69914300
-1.67709000
-0.99004400
-0.81079500
-2.32438300
-3.38460500
-1.83846500
-2.09273700
-1.80472300
-2.97845700
-1.58608100
-0.58743800
0.38214100

$-0.95395000$

$-0.42277500$

0.28320900

$-1.79294600$

$-2.17253700$

$-1.69041800$

$-2.78698500$

$-3.76392200$

$-2.95563300$

$-3.68279400$

$-3.34076600$

$-2.24328900$

$-2.95026300$

$-2.13674100$

$-0.86638100$

$-0.50526100$

$-1.08634200$

$-1.84012900$

0.12044800

1.10679400

0.25215000

$-0.60175000$

0.41208500

0.75440000

1. 53495300

0.10559200

$-0.92406600$

$-1.44472900$

$-1.31155100$

1.08238100

0.79863900

$-2.51684900$

$-2.60183300$

2.06597300

1.87917100

0.97616700

0.39152500

2.21546200
$-0.67244300$

$-3.12525500$

$-1.86508200$

1.25370000

0.94706400

0.53820100

2. 35214000

2. 43539100

3. 31012600

1.93069800

2. 76148400

1. 44904300

1.13025600

2. 16187100

2.06579800

3.18162300

1. 92633100

2.66378000

2.08561900

3.10785700

1. 92887900

1.06131600

1.17235100

1. 31551900

0.61433900

2.33318000

$-0.36308000$

$-1.11104100$

$-0.54625200$

$-0.52996700$

$-1.54491000$

$-0.30715700$

$-1.01662300$

0.50075200

0.38595500

0.31835400

0.24805600

0.80113300

0.12335600

0.52569100

$-1.05043900$

$-1.55339800$

$-2.45397500$

$-0.91467700$

2.13628800

2. 41347900

$-1.45512600$

$-0.90042400$

0.34295500

1. 21781800

$-1.92772100$

$-1.95951100$

$-0.98105500$

\section{S106}


2.19421800 3.52151400 4.38836300

3.64313200

3.50818300

$-3.81442800$

$-4.68738600$

$-3.80913800$

$-3.94693600$

$-2.38996300$

$-2.29973000$

$-3.28512100$

$-1.52613100$

0.56660700

$-0.52556700$

0.85399100

0.99036800

2. 62069700

3.04291500

2. 98458300

3.02369200

0.38998600

1.06766100

1.60894300

1.20457800

3.10784600

3.41477900

3.64667000

3.33352400

4.66635000

4.29715900

5.58920900

4.92744900
$-0.56737400$

$-1.74706800$

$-1.07935800$

$-2.52742800$

$-2.22607100$

$-1.22140300$

$-1.53759600$

$-1.79759000$

$-0.16672900$

$-2.94467600$

$-3.58247800$

$-3.26616400$

$-3.12936200$

3.23956900

3.32402000

3.02968500

4. 21286300

2.07725900

3.04659000

1.85203900

1.31969800

$-1.56387700$

$-2.97503700$

$-3.79970400$

$-2.92357700$

0.61199900

$-0.21620900$

1. 82696100

2.65610600

2.14976800

2.92025000

2.54776300

1. 26443900 


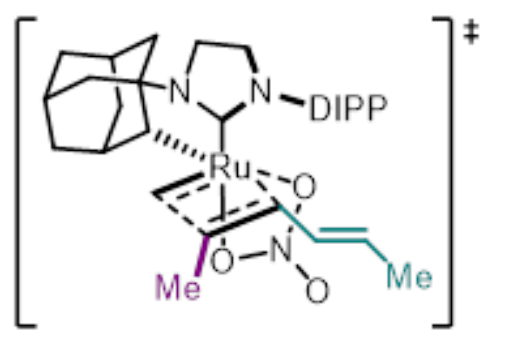

\section{6-TS}

SCF Energy (M06, n-octanol):

$-1731.659119$

SCF Energy (B3LYP, gas-phase):

$-1731.339581$

Enthalpy Correction:

0.787374

Free-Energy Correction:

0.682014

\begin{tabular}{|c|c|}
\hline $\mathrm{Ru}$ & -0.58028300 \\
\hline 0 & -1.82401300 \\
\hline O & -1.83818900 \\
\hline $\mathrm{N}$ & -0.64640000 \\
\hline $\mathrm{N}$ & 1.47620600 \\
\hline C & 0.22704000 \\
\hline C & -0.0477990 \\
\hline $\mathrm{H}$ & -0.41152100 \\
\hline $\mathrm{H}$ & -0.27431500 \\
\hline C & 1.43493700 \\
\hline $\mathrm{H}$ & 2.08799100 \\
\hline $\mathrm{H}$ & 1.76807500 \\
\hline C & -2.09047300 \\
\hline C & -2.6586210 \\
\hline $\mathrm{H}$ & -2.12120200 \\
\hline $\mathrm{H}$ & -2.49947300 \\
\hline C & -4.16573100 \\
\hline $\mathrm{H}$ & -4.55631000 \\
\hline C & -4.91179900 \\
\hline $\mathrm{H}$ & -4.8014900 \\
\hline $\mathrm{H}$ & -5.98661600 \\
\hline C & -4.34899100 \\
\hline $\mathrm{H}$ & -4.88231900 \\
\hline C & -2.85161400 \\
\hline $\mathrm{H}$ & -2.42738500 \\
\hline $\mathrm{H}$ & -2.74795800 \\
\hline $\mathrm{C}$ & -4.5277410 \\
\hline $\mathrm{H}$ & -4.16517200 \\
\hline $\mathrm{H}$ & -5.59723800 \\
\hline C & -3.75592500 \\
\hline $\mathrm{H}$ & -3.88622600 \\
\hline $\mathrm{C}$ & -2.26536200 \\
\hline $\mathrm{H}$ & -2.0359720 \\
\hline $\mathrm{C}$ & -4.35651000 \\
\hline $\mathrm{H}$ & -3.89349400 \\
\hline $\mathrm{H}$ & -5.42878500 \\
\hline C & 2.73500900 \\
\hline C & 3.03397900 \\
\hline $\mathrm{C}$ & 4.29731900 \\
\hline $\mathrm{H}$ & 4.54384300 \\
\hline C & 5.25108400 \\
\hline C & 4.95704600 \\
\hline $\mathrm{H}$ & 5.71232100 \\
\hline C & 3.70508200 \\
\hline C & 2.05392200 \\
\hline $\mathrm{H}$ & 1.0405550 \\
\hline C & 3.45722400 \\
\hline $\mathrm{H}$ & 2.38799900 \\
\hline C & 1.01445700 \\
\hline $\mathrm{H}$ & 1.37367300 \\
\hline 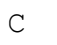 & 0.3037460 \\
\hline
\end{tabular}

$$
\begin{aligned}
& 0.53526700 \quad-0.81668400 \\
& 1.83668300 \quad 0.64905500 \\
& 2.26236600-1.48251800 \\
& -2.09926800 \quad 0.37781900 \\
& -1.56128700 \quad 0.42822800 \\
& -1.10329800 \quad 0.07205900 \\
& -3.18749800 \quad 1.15306800 \\
& -4.16430700 \quad 0.82464000 \\
& -3.07724800 \quad 2.22381100 \\
& -2.98035700 \quad 0.84933200 \\
& -3.15372400 \quad 1.70700800 \\
& -3.62707800 \quad 0.02771600 \\
& -1.86086000 \quad 0.28883000 \\
& -1.39609400 \quad 1.65405500 \\
& -0.49897400 \quad 1.98190000 \\
& -2.17870100 \quad 2.41072600 \\
& -1.09209800 \quad 1.52104900 \\
& -0.78106500 \quad 2.49914100 \\
& -2.35271300 \quad 1.04111200 \\
& -3.16829100 \quad 1.77084800 \\
& -2.14214100 \quad 0.95749800 \\
& -2.78627900-0.32747400 \\
& -3.67892800-0.68132800 \\
& -3.13177600-0.16054000 \\
& -3.49515800-1.10625500 \\
& \begin{array}{ll}
-3.94080100 & 0.57857500
\end{array} \\
& -1.63692500-1.34560000 \\
& -1.94863000 \quad-2.33486900 \\
& -1.40708700 \quad-1.45497700 \\
& -0.37670500-0.86240300 \\
& 0.43036500-1.59236700 \\
& -0.77689500-0.78764100 \\
& -1.25960000 \quad-1.74659700 \\
& 0.04415800 \quad 0.49817500 \\
& 0.95866700 \quad 0.87105900 \\
& 0.25022200 \quad 0.37081100 \\
& -0.87011900 \quad 0.47635600 \\
& -0.06033300 \quad 1.60304300 \\
& 0.54060800 \quad 1.66760300 \\
& 1.17422600 \quad 2.51332100 \\
& 0.33340500 \quad 0.67504700 \\
& -0.49169700 \quad-0.40438400 \\
& -0.66250000-1.16599400 \\
& -1.10978800 \quad-0.52701700 \\
& 0.11963500 \quad 2.76444700 \\
& \begin{array}{ll}
-0.01419500 & 2.37437400
\end{array} \\
& -2.04662800-1.70839500 \\
& -2.28149300 \quad-1.73124100 \\
& 2.23011700-0.32457000 \\
& 1.77847700 \quad 0.59320500 \\
& 0.17390500-2.43698100
\end{aligned}
$$

\section{S108}




\begin{tabular}{|c|c|c|c|}
\hline $\mathrm{H}$ & 1.08943800 & -0.55315800 & -2.66607000 \\
\hline C & 1.54862400 & 1.72276200 & -1.53549400 \\
\hline $\mathrm{H}$ & 2.33600900 & 0.98772500 & -1.41122600 \\
\hline $\mathrm{C}$ & 1.66724800 & 2.60002300 & -2.76994000 \\
\hline $\mathrm{H}$ & 2.36797900 & 3.42276300 & -2.57043800 \\
\hline $\mathrm{H}$ & 2.05008300 & 2.03540200 & -3.62488600 \\
\hline $\mathrm{H}$ & 0.70880800 & 3.04091800 & -3.05797600 \\
\hline $\mathrm{C}$ & 4.23425400 & -3.36968500 & -1.53357700 \\
\hline $\mathrm{H}$ & 4.00449100 & -4.06164100 & -2.35257300 \\
\hline $\mathrm{H}$ & 5.31589600 & -3.19019700 & -1.54016600 \\
\hline $\mathrm{H}$ & 3.98989700 & -3.86700700 & -0.58898200 \\
\hline C & 3.81735600 & -1.41080800 & -3.06590500 \\
\hline $\mathrm{H}$ & 4.89343900 & -1.22398200 & -3.15440400 \\
\hline $\mathrm{H}$ & 3.53537600 & -2.08546800 & -3.88255500 \\
\hline $\mathrm{H}$ & 3.29987000 & -0.45886600 & -3.22162400 \\
\hline C & 2.28776900 & -0.95324100 & 3.85127600 \\
\hline $\mathrm{H}$ & 2.18026300 & -1.97002800 & 3.46168800 \\
\hline $\mathrm{H}$ & 3.29668300 & -0.86371100 & 4.27166100 \\
\hline $\mathrm{H}$ & 1.56859300 & -0.82972400 & 4.66958200 \\
\hline C & 2.11568500 & 1.51445400 & 3.41552300 \\
\hline $\mathrm{H}$ & 1.27490400 & 1.63504700 & 4.10751500 \\
\hline $\mathrm{H}$ & 3.03450000 & 1.65408600 & 3.99728000 \\
\hline $\mathrm{H}$ & 2.06254500 & 2.31926100 & 2.67623100 \\
\hline $\mathrm{H}$ & 6.22613000 & 0.80752600 & 0.74965900 \\
\hline $\mathrm{N}$ & -2.27198600 & 2.56306500 & -0.30864200 \\
\hline 0 & -3.06086000 & 3.47596500 & -0.12233500 \\
\hline C & 0.47823100 & 3.58257900 & -0.20767600 \\
\hline $\mathrm{H}$ & 0.31781300 & 4.14139100 & -1.12659400 \\
\hline $\mathrm{C}$ & 0.15376900 & 4.16802900 & 0.95908300 \\
\hline $\mathrm{H}$ & 0.26936400 & 3.60037700 & 1.88202000 \\
\hline C & -0.42655300 & 5.54213100 & 1.09312200 \\
\hline $\mathrm{H}$ & -1.45386900 & 5.48262200 & 1.47705000 \\
\hline $\mathrm{H}$ & 0.14706600 & 6.15656600 & 1.80050500 \\
\hline $\mathrm{H}$ & -0.45943300 & 6.06183000 & 0.12991700 \\
\hline $\mathrm{H}$ & -0.04257000 & 0.70872800 & -3.33143200 \\
\hline
\end{tabular}

\section{Transition states with larger substrate models}

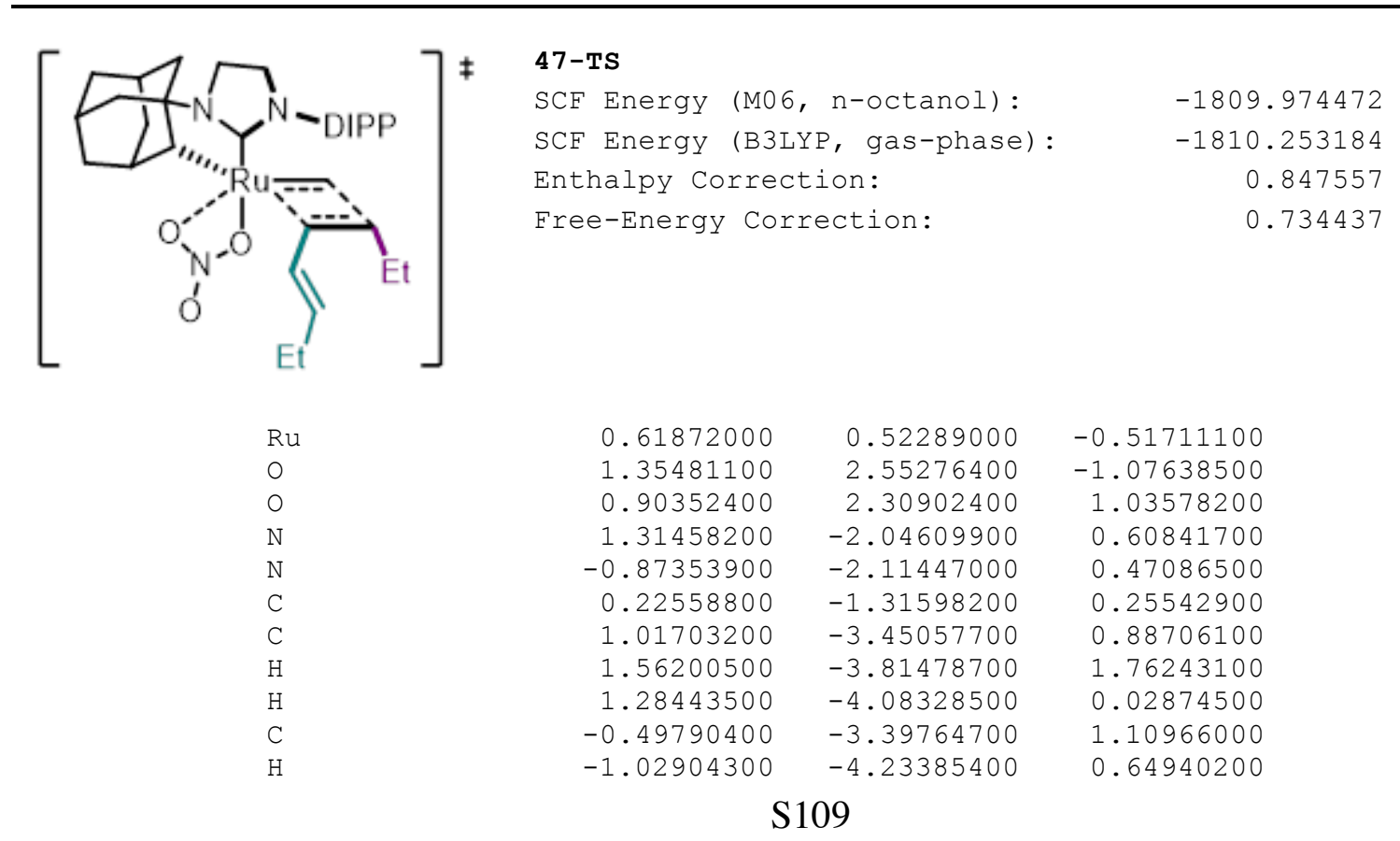




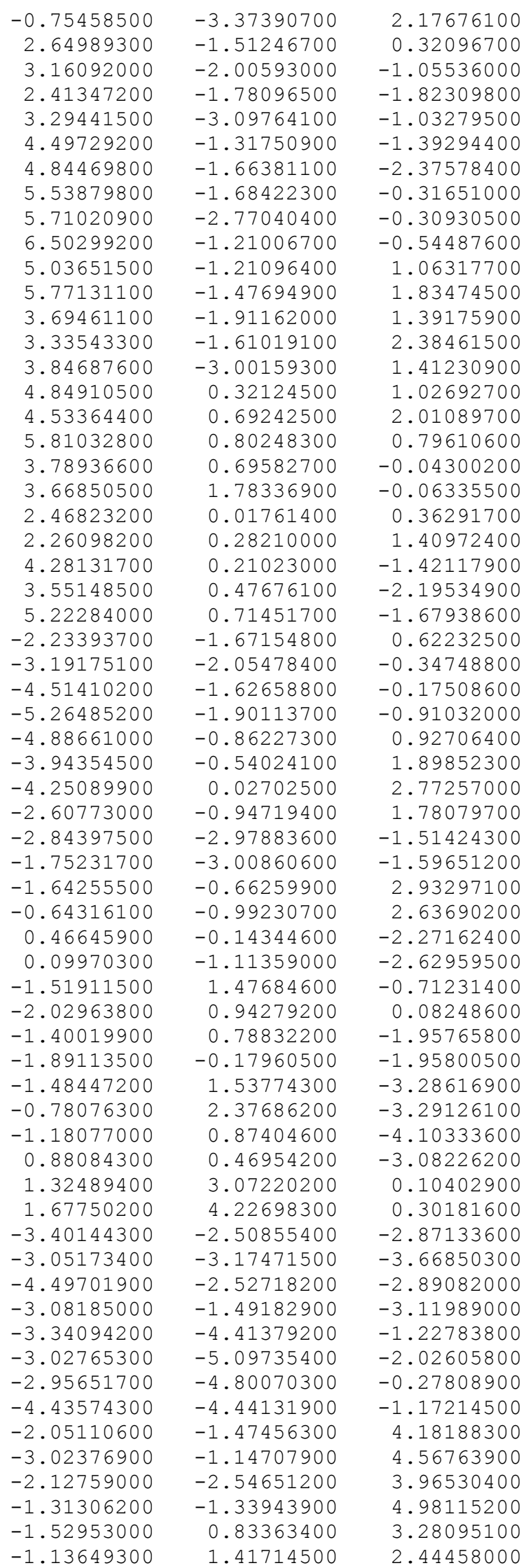

\section{S110}




$\begin{array}{rrrr}\mathrm{H} & -2.49776700 & 1.25468800 & 3.57794500 \\ \mathrm{H} & -0.84269000 & 0.96666700 & 4.12486500 \\ \mathrm{H} & -5.91665800 & -0.53413300 & 1.03848400 \\ \mathrm{C} & -1.55065600 & 2.93362100 & -0.60557200 \\ \mathrm{H} & -1.08315700 & 3.51146100 & -1.39933000 \\ \mathrm{C} & -2.10887400 & 3.59285900 & 0.42545900 \\ \mathrm{H} & -2.56076600 & 3.01686800 & 1.23491300 \\ \mathrm{C} & -2.11999100 & 5.08465300 & 0.59227600 \\ \mathrm{H} & -1.71140100 & 5.56057000 & -0.30785200 \\ \mathrm{H} & -3.15803200 & 5.43661000 & 0.69559400 \\ \mathrm{C} & -2.91221800 & 2.04141700 & -3.56228200 \\ \mathrm{H} & -3.24256300 & 2.74521400 & -2.79216000 \\ \mathrm{H} & -2.95692800 & 2.54870700 & -4.53292200 \\ \mathrm{H} & -3.62717800 & 1.21007400 & -3.58643400 \\ \mathrm{C} & -1.31251900 & 5.53438500 & 1.82577100 \\ \mathrm{H} & -1.35631700 & 6.62378800 & 1.94057400 \\ \mathrm{H} & -1.71073400 & 5.08371200 & 2.74321200 \\ \mathrm{H} & -0.26505100 & 5.23446300 & 1.72563600\end{array}$

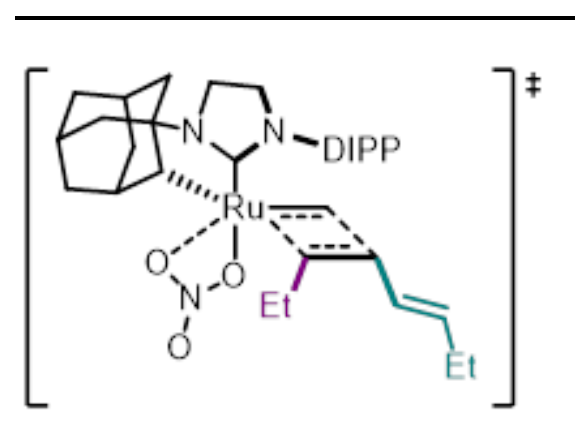

\section{8-TS}

SCF Energy (M06, n-octanol):

SCF Energy (B3LYP, gas-phase):

Enthalpy Correction:

Free-Energy Correction:

\author{
$-1809.971257$ \\ $-1810.248762$ \\ 0.847613 \\ 0.733726
}

$$
\begin{array}{r}
0.61801500 \\
1.36145600 \\
1.34313100 \\
1.34401300 \\
-0.83914800 \\
0.24673200 \\
1.03459100 \\
1.68299900 \\
1.15409800 \\
-0.42868400 \\
-1.05393700 \\
-0.52953900 \\
2.64820900 \\
2.94714800 \\
2.11594400 \\
3.03105400 \\
4.25826700 \\
4.45451100 \\
5.41503200 \\
5.53380400 \\
6.36188800 \\
5.12387000 \\
5.94125500 \\
3.80608700 \\
3.59742100 \\
3.90537300 \\
5.00491800 \\
4.83588200 \\
5.94842400 \\
3.83393600 \\
3.77025000
\end{array}
$$

0.63750400

2.56116600

0.96852700

$-1.94299500$

$-2.04943200$

$-1.25479900$

$-3.15186700$

$-3.98604500$

$-2.97264100$

$-3.38047800$

$-3.69057400$

$-4.12860700$

$-1.27500500$

$-0.61374500$

0.04324100

$-1.38747700$

0.19147000

0.66791000

$-0.76668800$

$-1.52285400$

$-0.21277700$

$-1.44625300$

$-2.13555600$

$-2.25115800$

$-2.76576700$

$-3.02221400$

$-0.36040300$

$-0.82058500$

0.19993900

0.60099400

1. 36781500
0.29723200

1.10574100

2.58688900

$-0.79886200$

$-0.67273700$

$-0.39181100$

$-1.56206900$

$-1.28065400$

$-2.64015000$

$-1.17636500$

$-2.01674200$

$-0.37911700$

$-0.79316600$

$-2.16294300$

$-2.44098600$

$-2.94034600$

$-2.07574400$

$-3.04576100$

$-1.72539000$

$-2.51486500$

$-1.66849200$

$-0.37120600$

$-0.12079200$

$-0.46966000$

0.47763200

$-1.24886800$

0.71861400

1.70112900

0.78529100

0.37929700

1.15675000

\section{S111}




$$
\begin{aligned}
& 2.54467900 \\
& 2.47148300 \\
& \text { 4. } 11519500 \\
& \text { 3. } 30145200 \\
& 5.03716000 \\
& -2.16936700 \\
& -3.22436200 \\
& -4.51795300 \\
& -5.34253600 \\
& -4.76877700 \\
& -3.72840200 \\
& -3.93884700 \\
& -2.41550100 \\
& -3.00724600 \\
& -1.92788700 \\
& -1.32818700 \\
& -0.37058900 \\
& 0.11144600 \\
& -0.39951600 \\
& -1.39303700 \\
& -1.88395000 \\
& -1.54020500 \\
& -2.16520800 \\
& 0.43509100 \\
& \text { 1. } 63106900 \\
& \text { 2. } 12892700 \\
& -3.64932500 \\
& -3.41661000 \\
& -4.74097100 \\
& -3.28104400 \\
& -3.53472700 \\
& -3.31028000 \\
& -3.09028700 \\
& -4.62192300 \\
& -1.60079900 \\
& -2.52423500 \\
& -1.70396800 \\
& -0.77927400 \\
& -1.16680100 \\
& -0.87373900 \\
& -2.09133800 \\
& -0.38447400 \\
& -5.77907900 \\
& -1.58542000 \\
& -0.89818500 \\
& -2.41930200 \\
& -3.09431700 \\
& -2.50661800 \\
& -3.52071500 \\
& -1.82281700 \\
& -1.45613000 \\
& -0.97728900 \\
& -0.90539600 \\
& -2.91710400 \\
& -3.49550000 \\
& -2.95717800 \\
& -3.41150900 \\
& -2.19227200 \\
& -2.87101600 \\
& -2.29882400 \\
& -1.16777500
\end{aligned}
$$

\section{S112}




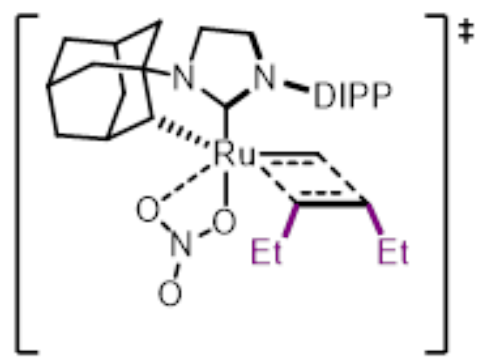

\section{9-TS}

SCF Energy (M06, n-octanol): $\quad-1732.570489$

SCF Energy (B3LYP, gas-phase): $\quad-1732.890950$

Enthalpy Correction:

0.812054

Free-Energy Correction:

0.705038

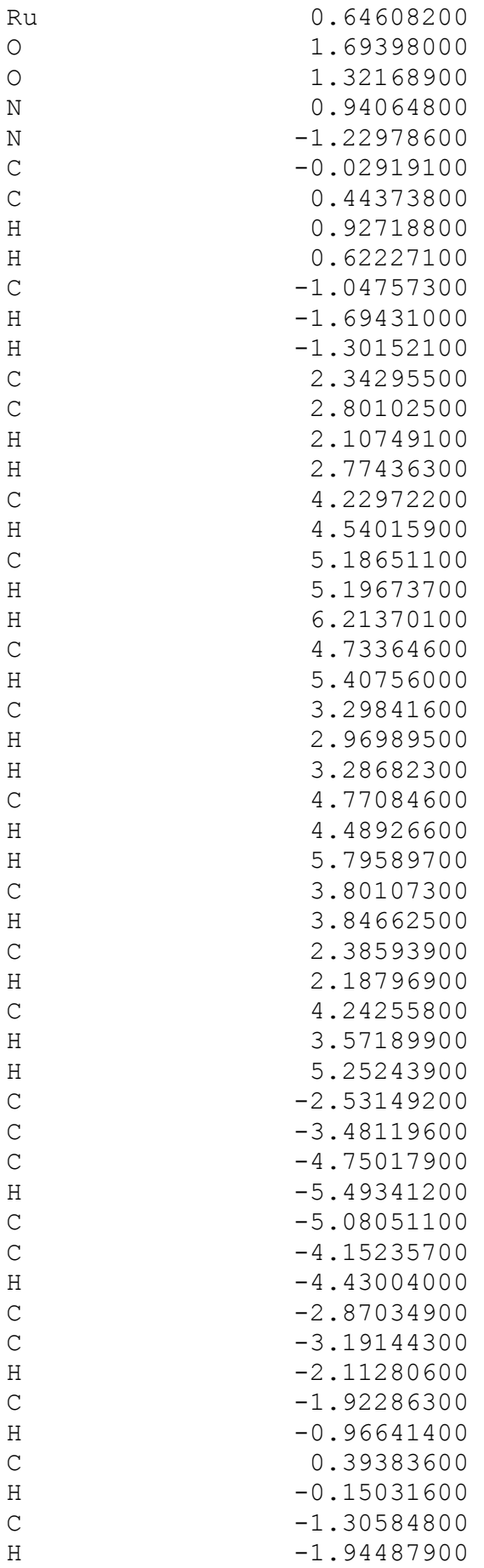

$$
0.80557900
$$

2.75897200

2.02608400

$-2.06744400$

$-1.78769200$

$-1.11859100$

$-3.43212300$

$-4.13103100$

$-3.78159200$

$-3.24567000$

$-3.80914600$

$-3.52632600$

$-1.66597200$

$-1.74867800$

$-1.17849300$

$-2.79394100$

$-1.18661100$

$-1.23684800$

$-2.03369300$

$-3.07418800$

$-1.65520500$

$-1.97575200$

$-2.58437200$

$-2.54210300$

$-2.53509900$

$-3.58798000$

$-0.50814900$

$-0.44182300$

$-0.11974000$

0.34839300

1. 38523300

$-0.22413800$

$-0.28090700$

0.27976100

0.88931300

0.69880300

$-1.24731200$

$-1.17005600$

$-0.65668400$

$-0.58071100$

$-0.25266500$

$-0.38775500$

$-0.10004100$

$-0.90029700$

$-1.70533700$

$-1.88073100$

$-1.11147000$

$-1.47582300$

0.74319900

0.01190400

1.96394000

1.20307900
$-0.15302600$

$-0.18516100$

1.82797000

0.08056500

0.01239500

0.02507400

$-0.09518500$

0.59275500

$-1.12217400$

0.19668000

$-0.47978200$

1.22747500

$-0.06597000$

$-1.54391700$

$-2.17106400$

$-1.88543200$

$-1.67789000$

$-2.73031300$

$-0.81455600$

$-1.17021200$

$-0.90472600$

0.65918200

1.27677100

0.78115900

1.82891600

0.43858300

1.13577900

2.19508400

1.05242300

0.27916000

0.62512000

0.47211100

1.55235600

$-1.19637300$

$-1.81417400$

$-1.30538700$

0.29478700

$-0.75182800$

$-0.45534200$

$-1.24382200$

0.83523700

1.86381600

2.87400900

1.62498700

$-2.15337600$

$-2.22212600$

2.80651600

2. 42190400

$-2.01316100$

$-2.62301300$

0.14682600

0.58186300

\section{S113}


$-1.25079800$

$-1.89416900$

0.92267700

1. 83779500

2.43287200

$-3.57741800$

$-3.29942600$

$-4.65650300$

$-3.07146600$

$-3.90710200$

$-3.63931900$

$-3.64572900$

$-4.99615100$

$-2.48206000$

$-3.41763300$

$-2.68816300$

$-1.76345600$

$-1.62314000$

$-1.12256200$

$-2.53672200$

$-0.95872100$

$-6.06872200$

$-1.25829800$

$-0.83005300$

$-0.60890800$

$-1.02429700$

$-0.01601400$

$-1.71227900$

$-2.67206600$

$-2.63351700$

$-3.13604200$

$-3.33267600$

$-1.27491800$

$-1.15179500$

$-0.58300100$

$-2.29575000$
1.96345600

1.22881200

1.48885800

2.92850900

3.89547400

$-0.73250900$

$-1.15892700$

$-0.54285800$

0.23283300

$-3.05747500$

$-3.48196500$

$-3.78859100$

$-2.93168100$

$-2.18921200$

$-1.86094300$

$-3.12972200$

$-2.39533300$

0.18764000

0.92357700

0.63985700

$-0.02521700$

0.14884900

3.24422900

3.03844200

3. 98712400

3. 24813600

3.62949100

4.00863100

3.82963100

4.73273500

4.09798900

3.10795300

3.11348100

4.08150700

2.40741200

2.76579500
$-1.27435000$

$-1.75174900$

$-2.61976400$

1.08851900

1.53806500

$-3.28285600$

$-4.25372800$

$-3.30806800$

$-3.18495400$

$-2.37071200$

$-3.34564000$

$-1.59800900$

$-2.34693000$

3. 76046000

4.22826700

3.23621100

4.56217200

3.57815600

2. 94361800

3. 98189100

4. 42362800

1.04335700

0.95966100

1.94397800

0.48846000

$-2.06572100$

$-1.87282000$

$-1.67072300$

1.14791900

1.76796400

0.19126400

1.64304600

$-3.57177200$

$-4.06952000$

$-4.04332100$

$-3.77488700$

\section{S114}




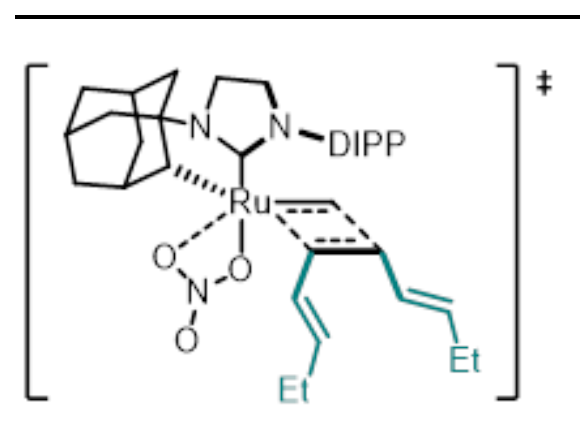

50-TS

SCF Energy (M06, n-octanol): $\quad-1887.373081$

SCF Energy (B3LYP, gas-phase): $\quad-1887.609875$

Enthalpy Correction:

0.883151

Free-Energy Correction:

0.763473

$$
\begin{array}{rr}
0.59387200 & -0.07721800 \\
2.72155200 & 0.27393900 \\
1.52603900 & 2.08514200 \\
-2.12360200 & -0.38177600 \\
-2.33554300 & -0.32270100 \\
-1.42589000 & -0.22875000 \\
-3.51104700 & -0.79817800 \\
-4.19433400 & -0.27354200 \\
-3.62122200 & -1.87839500 \\
-3.72303700 & -0.42651900 \\
-4.28911800 & -1.17757200 \\
-4.23465800 & 0.53880500 \\
-1.39152500 & -0.47374200 \\
-1.10148300 & -1.94810400 \\
-0.60197400 & -2.44509900 \\
-2.04732800 & -2.47857600 \\
-0.21378400 & -1.99857100 \\
-0.00336000 & -3.04671400 \\
-0.95888600 & -1.32401000 \\
-1.88980200 & -1.86668600 \\
-0.34639800 & -1.36047900 \\
-1.26721300 & 0.14225100 \\
-1.80445800 & 0.62483200 \\
-2.16088300 & 0.17979000 \\
-2.41649000 & 1.21540000 \\
-3.10293500 & -0.35160700 \\
0.06219700 & 0.88228900 \\
-0.12610000 & 1.94043800 \\
0.68366000 & 0.85328100 \\
0.81080900 & 0.21740600 \\
1.75245600 & 0.74826000 \\
-0.09878900 & 0.32938300 \\
-0.39360800 & 1.38261900 \\
1.10696100 & -1.25306400 \\
1.64595400 & -1.73644800 \\
-1.75944200 & -1.29853000 \\
-2.15743300 & 0.18191700 \\
-2.12577300 & -0.73162000 \\
-1.95206900 & -0.21968600 \\
-1.91374200 & -0.90131800 \\
-1.83508700 & 1.14937800 \\
-1.92908700 & 2.03702400 \\
-1.87421800 & 3.10560900 \\
-2.10936000 & 1.58102500 \\
-2.35920200 & -2.22878000 \\
-12800 & -2.44493700 \\
-18000 & 2.06361000 \\
-1.0682900 \\
-1.92355600
\end{array}
$$

\section{S115}




\begin{tabular}{|c|c|c|}
\hline & & \\
\hline $\begin{array}{l}-0.15715900 \\
-1.55961300\end{array}$ & $\begin{array}{l}0.05542700 \\
1.27857800\end{array}$ & $\begin{array}{r}-2.64326600 \\
0.38227600\end{array}$ \\
\hline-2.00575000 & 0.36798400 & 0.76704700 \\
\hline-1.53956900 & 1.39523100 & -1.05731200 \\
\hline-2.07696500 & 0.59366700 & -1.55547400 \\
\hline 0.55090100 & 1.71417100 & -2.41175600 \\
\hline 1.26261800 & 2.66389100 & 1.56026100 \\
\hline 1.55946400 & 3.65085200 & 2.21937700 \\
\hline-3.64948700 & -1.37198500 & -3.12202900 \\
\hline-3.39365200 & -1.54199200 & -4.17427100 \\
\hline-4.73374400 & -1.49988700 & -3.02738400 \\
\hline-3.41697500 & -0.32958500 & -2.88370500 \\
\hline-3.27527300 & -3.80459300 & -2.60256400 \\
\hline-3.06641200 & -4.00073700 & -3.66104700 \\
\hline-2.73683300 & -4.54827000 & -2.00560500 \\
\hline-4.34705700 & -3.96552600 & -2.43561400 \\
\hline-1.29775300 & -3.64889500 & 3.35959700 \\
\hline-2.21043400 & -3.62146000 & 3.96646800 \\
\hline-1.38489800 & -4.49935000 & 2.67314900 \\
\hline-0.45495000 & -3.83960100 & 4.03427600 \\
\hline-0.94452400 & -1.15195900 & 3.59908300 \\
\hline-0.69329200 & -0.21613200 & 3.09355200 \\
\hline-1.86306800 & -1.00062600 & 4.17916200 \\
\hline-0.14009500 & -1.36901900 & 4.31150400 \\
\hline-5.59504300 & -1.69191300 & 1.52562800 \\
\hline-1.68444100 & 2.44066900 & 1.25903700 \\
\hline-1.34734900 & 3.40257400 & 0.88177600 \\
\hline-2.18734800 & 2.38670200 & 2.50502900 \\
\hline-2.51472200 & 1.42432300 & 2.90143900 \\
\hline-2.27733500 & 3.55704700 & 3.44116700 \\
\hline-2.06913700 & 4.48478800 & 2.89389300 \\
\hline-3.30208000 & 3.64110000 & 3.83320600 \\
\hline-1.64987700 & 2.71868100 & -1.71827800 \\
\hline-1.05576800 & 3.52632600 & -1.29503000 \\
\hline-2.42716000 & 2.95689800 & -2.78296100 \\
\hline-3.00596400 & 2.13591600 & -3.21326900 \\
\hline-2.57092600 & 4.28930800 & -3.46593700 \\
\hline-3.62123800 & 4.61388400 & -3.41576000 \\
\hline-1.98540500 & 5.04446300 & -2.92677500 \\
\hline-1.29350800 & 3.42754200 & 4.62143800 \\
\hline-0.26280000 & 3.39398100 & 4.25518200 \\
\hline-1.39533200 & 4.27797400 & 5.30592400 \\
\hline-1.48139800 & 2.51090200 & 5.19385600 \\
\hline-2.13792100 & 4.25053200 & -4.94238700 \\
\hline-2.71755000 & 3.50926800 & -5.50613500 \\
\hline-2.28681100 & 5.22525200 & -5.42087300 \\
\hline-1.07869500 & 3.98483100 & -5.03286700 \\
\hline
\end{tabular}

\section{S116}




\section{Other Vinylcarbene Complexes}

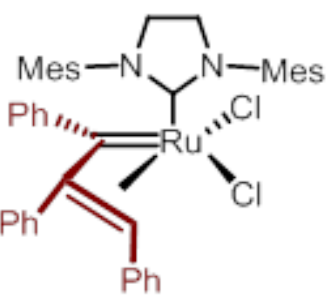

$\mathrm{Ru}$

$\mathrm{Cl}$

$\mathrm{Cl}$

$\mathrm{N}$

$\mathrm{N}$

C

C

$\mathrm{H}$

$\mathrm{H}$

C

$\mathrm{C}$

$\mathrm{C}$

C
31

SCF Energy (M06, n-octanol): $\quad-2749.758820$

SCF Energy (B3LYP, gas-phase): $\quad-2749.584244$

Enthalpy Correction:

0.779107

Free-Energy Correction:
0.651433

$$
\begin{array}{r}
0.51251200 \\
0.91954700 \\
1.44474700 \\
0.04260000 \\
2.12706100 \\
-1.32017000 \\
-1.11020200 \\
-0.26658900 \\
-2.54340600 \\
-2.52781100 \\
-1.57128700
\end{array}
$$

$-3.72119200$

$-3.69414000$

$-4.94809800$

$-5.87663200$

$-4.97817100$

$-5.92828300$

$-3.78900100$

$-3.81436100$

$-1.67687600$

$-2.28367000$

$-2.26012400$

$-2.92124900$

$-3.38837900$

$-2.95861800$

$-3.45137700$

$-2.35966200$

$-2.37836900$

$-1.72972700$

$-1.24622100$

0.43065600

0.57295400

0.14771600

1. 23843100

1. 32881800

1.79024900

2.31458900

1. 67545300

2. 11969300

1.00421100

0.95256900

0.84851200

$-1.39081000$

$-2.14863900$

$-3.53658300$

$-4.12732800$

$-4.17412500$

$-3.38301100$

$-3.85867900$

$-1.99945500$
$-0.02714000$

$-1.71801800$

1.57026700

$-2.07116400$

$-1.80261400$

0.21459100

1.50556000

1.52998500

$-0.25895300$

$-1.16725900$

$-1.50942500$

$-1.59499800$

$-2.28458100$

$-1.13719100$

$-1.47136900$

$-0.23970800$

0.12795500

0.19976500

0.90304200

2.73864200

3.69946700

3.56793100

4.81142700

5.54112500

4.98522100

5.85415100

4.03620700

4.16608300

2.91724000

2.19748400

2.70870700

2.83375400

2.07159700

3.92356000

4.00462500

4.90403800

5.75201700

4.78126100

5.52990100

3.69813300

3.58771200

$-1.43751600$

$-2.20010400$

$-1.55498200$

$-1.75254300$

$-1.24491400$

$-2.58589200$

$-3.25754500$

$-3.93074900$

$-3.09293700$
$-0.77498600$

$-2.44297300$

$-2.29611400$

1.60591600

0.97559600

$-0.66641400$

$-0.01139500$

1. 10052300

$-1.29323900$

$-2.37221700$

$-2.75199500$

$-2.94699800$

$-3.78637500$

$-2.45633800$

$-2.91221500$

$-1.38482400$

$-1.00613600$

$-0.81036700$

0.01550500

$-0.64297200$

0.18531900

1. 26299700

$-0.36226600$

0.29397900

$-1.74741200$

$-2.17571400$

$-2.57842900$

$-3.65704300$

$-2.03474200$

$-2.68500800$

1. 64486600

3.03944500

3.68876700

3.59989600

4.68007900

2.77307400

3.20560000

1. 38466200

0.73416500

0.82192900

$-0.25614400$

0.70853800

1. 59680400

2.58958500

2. 60483200

3. 36516700

1.68771800

0.74714400

0.03766300

0.68515900

\section{S117}




$\begin{array}{rrr}-1.53441100 & -0.69355100 & 3.67123200 \\ -1.77102400 & -1.09647200 & 4.66390200 \\ -1.93371200 & 0.32708700 & 3.63786700 \\ -0.44758900 & -0.62918300 & 3.58643000 \\ -5.67352000 & -2.76394700 & 1.69659900 \\ -6.12514000 & -2.33469200 & 2.59667600 \\ -5.95254700 & -3.82314100 & 1.64898100 \\ -6.12625100 & -2.27141900 & 0.82665300 \\ -1.19268900 & -3.87755900 & -0.32032800 \\ -0.47023600 & -4.54217100 & 0.17209200 \\ -0.62455600 & -3.23013300 & -0.99593900 \\ -1.85128200 & -4.50602000 & -0.92687700 \\ 3.32598300 & -1.37765600 & 0.29491500 \\ 3.91896100 & -0.14101900 & 0.59965000 \\ 5.09974100 & 0.21026300 & -0.06953300 \\ 5.55239200 & 1.17515400 & 0.14966800 \\ 5.70506000 & -0.63523600 & -0.99586200 \\ 5.12088400 & -1.88773700 & -1.22751800 \\ 5.59308700 & -2.57283600 & -1.92812100 \\ 3.94395900 & -2.28496500 & -0.59636700 \\ 3.36451900 & 0.81142300 & 1.63095200 \\ 2.51887800 & 0.39138200 & 2.17880900 \\ 3.01965600 & 1.73898400 & 1.16394000 \\ 4.14096400 & 1.08040700 & 2.35743300 \\ 6.95050900 & -0.21718500 & -1.74050800 \\ 7.64906000 & -1.05352700 & -1.85740400 \\ 7.47343000 & 0.59541200 & -1.22564100 \\ 6.69922300 & 0.13950200 & -2.74774700 \\ 3.37058200 & -3.65466100 & -0.86642800 \\ 3.95985100 & -4.16852000 & -1.63152600 \\ 2.34053600 & -3.58069100 & -1.22537000 \\ 3.37804500 & -4.28891600 & 0.03054300 \\ 0.79457100 & -3.01825800 & 2.46184200 \\ 0.58046800 & -4.04785900 & 2.15086500 \\ 0.49654700 & -2.90789900 & 3.50798400 \\ 2.24600200 & -2.61575500 & 2.20097100 \\ 2.66667700 & -2.00378100 & 3.00982900 \\ 2.90938100 & -3.46838300 & 2.03859900 \\ -0.27651100 & 0.66498800 & 1.74937700 \\ & & \end{array}$

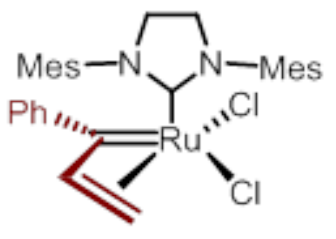

Ru
$\mathrm{Cl}$
$\mathrm{Cl}$
$\mathrm{N}$
$\mathrm{N}$
$\mathrm{C}$
$\mathrm{C}$
$\mathrm{C}$
$\mathrm{C}$
$\mathrm{C}$
$\mathrm{H}$
$\mathrm{C}$
$\mathrm{H}$
$\mathrm{C}$
32

SCF Energy (M06, n-octanol): SCF Energy (B3LYP, gas-phase): Enthalpy Correction:

Free-Energy Correction:

$$
\begin{array}{r}
-2287.894386 \\
-2287.486239 \\
0.607318 \\
0.499915
\end{array}
$$

$$
\begin{array}{rrr}
0.51402100 & -1.00638000 & -0.26107600 \\
0.61771200 & -1.28082700 & 2.14364800 \\
1.50896100 & -3.17000500 & -0.49812700 \\
-0.34384100 & 2.02966000 & -0.00029900 \\
1.81207900 & 1.65189600 & 0.13159100 \\
-1.25215100 & -1.25824100 & -0.77447500 \\
-0.65121900 & -1.45938100 & -2.07440900 \\
0.25997600 & -0.53745600 & -2.57419000 \\
-2.52725900 & -1.70196100 & -0.26711200 \\
-2.73213100 & -1.96174000 & 1.10500900 \\
-1.90229400 & -1.82249100 & 1.79045400 \\
-3.96754700 & -2.42138100 & 1.54882700 \\
-4.11328600 & -2.63617400 & 2.60377500 \\
-5.01701300 & -2.61728400 & 0.64402300
\end{array}
$$

\section{S118}




\begin{tabular}{|c|c|c|c|}
\hline $\mathrm{H}$ & -5.97840200 & -2.98064700 & 0.99792300 \\
\hline $\mathrm{C}$ & -4.82845800 & -2.35702500 & -0.71717600 \\
\hline $\mathrm{H}$ & -5.64039300 & -2.51586300 & -1.42166600 \\
\hline C & -3.59265800 & -1.90658800 & -1.17160500 \\
\hline $\mathrm{H}$ & -3.43741900 & -1.70623300 & -2.22803000 \\
\hline $\mathrm{C}$ & 0.60297800 & 1.05821600 & -0.01106100 \\
\hline C & -1.77586700 & 1.88972900 & 0.02858900 \\
\hline C & -2.51696600 & 2.09629400 & -1.14871500 \\
\hline $\mathrm{C}$ & -3.91345200 & 2.00860300 & -1.08150000 \\
\hline $\mathrm{H}$ & -4.48991300 & 2.16013600 & -1.99217300 \\
\hline C & -4.58092200 & 1.74034400 & 0.11325100 \\
\hline $\mathrm{C}$ & -3.81549100 & 1.59391300 & 1.27601900 \\
\hline $\mathrm{H}$ & -4.31843900 & 1.41252000 & 2.22320100 \\
\hline C & -2.42174800 & 1.67321300 & 1.26564300 \\
\hline $\mathrm{C}$ & -1.87754200 & 2.42017200 & -2.48056100 \\
\hline $\mathrm{H}$ & -2.42241800 & 3.22839300 & -2.98119500 \\
\hline $\mathrm{H}$ & -1.89916200 & 1.55388600 & -3.15278000 \\
\hline $\mathrm{H}$ & -0.83414800 & 2.72860200 & -2.37961600 \\
\hline $\mathrm{C}$ & -6.08364600 & 1.59753300 & 0.15494000 \\
\hline $\mathrm{H}$ & -6.56023700 & 2.10032700 & -0.69306400 \\
\hline $\mathrm{H}$ & -6.50197300 & 2.01440400 & 1.07772700 \\
\hline $\mathrm{H}$ & -6.37154900 & 0.53872700 & 0.11626600 \\
\hline $\mathrm{C}$ & -1.65092400 & 1.53739900 & 2.55618100 \\
\hline $\mathrm{H}$ & -1.06686200 & 2.44016600 & 2.77815300 \\
\hline $\mathrm{H}$ & -0.95044500 & 0.69535300 & 2.52969100 \\
\hline $\mathrm{H}$ & -2.33768100 & 1.38267400 & 3.39376500 \\
\hline C & 3.09729300 & 0.99834900 & 0.10516800 \\
\hline $\mathrm{C}$ & 3.73659800 & 0.74650500 & -1.12331900 \\
\hline $\mathrm{C}$ & 4.98444000 & 0.11157200 & -1.10474600 \\
\hline $\mathrm{H}$ & 5.47353600 & -0.10338100 & -2.05283600 \\
\hline $\mathrm{C}$ & 5.61603300 & -0.24337100 & 0.08650100 \\
\hline $\mathrm{C}$ & 4.98903200 & 0.09469700 & 1.29161500 \\
\hline $\mathrm{H}$ & 5.48347400 & -0.13905200 & 2.23163100 \\
\hline $\mathrm{C}$ & 3.74271400 & 0.72070600 & 1.33177500 \\
\hline $\mathrm{C}$ & 3.17771900 & 1.19091700 & -2.45602800 \\
\hline $\mathrm{H}$ & 2.17965300 & 1.62303200 & -2.37122800 \\
\hline $\mathrm{H}$ & 3.12100700 & 0.35231400 & -3.15760700 \\
\hline $\mathrm{H}$ & 3.83410100 & 1.94537400 & -2.90875200 \\
\hline C & 6.93651900 & -0.97557100 & 0.08175100 \\
\hline $\mathrm{H}$ & 7.57717200 & -0.65477000 & 0.91060600 \\
\hline $\mathrm{H}$ & 7.48215300 & -0.81600500 & -0.85415700 \\
\hline $\mathrm{H}$ & 6.78031100 & -2.05655300 & 0.19103200 \\
\hline C & 3.13348400 & 1.09792200 & 2.65955300 \\
\hline $\mathrm{H}$ & 3.81799600 & 0.84660300 & 3.47509400 \\
\hline $\mathrm{H}$ & 2.19410000 & 0.56180000 & 2.82563300 \\
\hline $\mathrm{H}$ & 2.92701100 & 2.17430200 & 2.72379900 \\
\hline $\mathrm{C}$ & 0.22441000 & 3.37337500 & 0.25772800 \\
\hline $\mathrm{H}$ & -0.08588600 & 3.72503000 & 1.24783100 \\
\hline $\mathrm{H}$ & -0.13954600 & 4.08882900 & -0.48575400 \\
\hline $\mathrm{C}$ & 1.73494100 & 3.12537300 & 0.16759400 \\
\hline $\mathrm{H}$ & 2.17875800 & 3.54268900 & -0.74492900 \\
\hline $\mathrm{H}$ & 2.28683600 & 3.52058300 & 1.02464100 \\
\hline $\mathrm{H}$ & 0.08840000 & 0.52738000 & -2.52168700 \\
\hline $\mathrm{H}$ & -0.59996100 & -2.49016900 & -2.43477500 \\
\hline $\mathrm{H}$ & 0.99063200 & -0.87680900 & -3.30215500 \\
\hline
\end{tabular}

\section{S119}




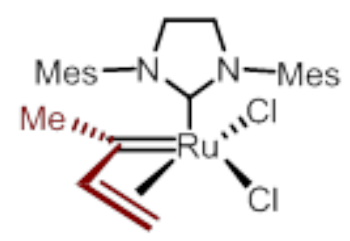

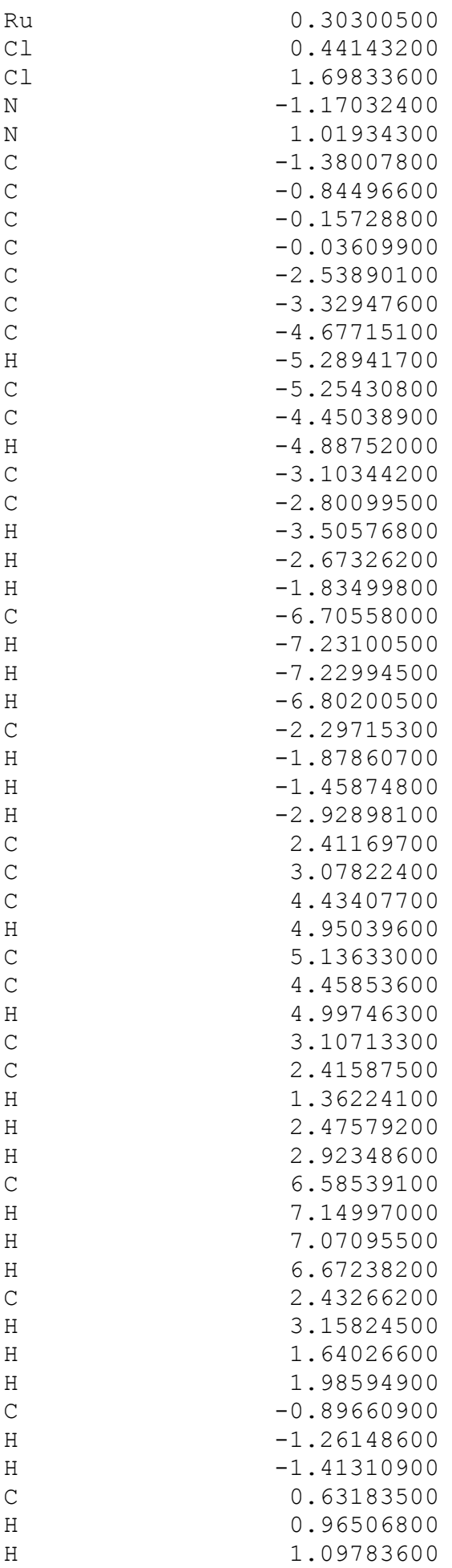

33

SCF Energy (M06, n-octanol): $\quad$-2096.250354

SCF Energy (B3LYP, gas-phase): $\quad-2095.746593$

Enthalpy Correction:

0.550615

0.449992

Free-Energy Correction: $\begin{array}{rr}1.25620200 & 0.00633800 \\ 1.07387700 & -2.39818800 \\ 3.19614300 & -0.10015800 \\ -1.52463800 & 0.25418400 \\ -1.63109200 & 0.17836500 \\ 1.90770600 & 0.31417300 \\ 2.28665500 & 1.60260100 \\ 1.36362500 & 2.37588300\end{array}$

$-0.78578300$

$-1.10112100$

$-0.91799900$

$-0.56612000$

$-0.41870100$

$-0.41751000$

$-0.65256100$

$-0.56865700$

$-1.00315600$

$-1.09977200$

$-1.68486300$

$-0.13409000$

$-1.60975400$

$-0.02779100$

$-0.03575000$

$-0.71119000$

0.98001100

$-1.27192500$

$-2.28645200$

$-0.57338100$

$-1.17787500$

$-1.25887500$

$-0.89621700$

$-0.55525900$

$-0.25770600$

$-0.59063900$

$-1.01877900$

$-1.08173100$

$-1.36412300$

$-0.90522000$

$-1.18435100$

0.08201200

$-1.61333600$

$-0.17201700$

$-0.79662600$

$-0.23286900$

0.86585300

$-1.84199000$

$-1.90780800$

$-1.14991900$

$-2.83703900$

$-2.98053600$

$-3.44602300$

$-3.44870700$

$-3.03673800$

$-3.35835600$

$-3.69101900$
0.14353800

0.11477700

1. 26322900

1.09847400

1.98596700

$-0.16217500$

$-1.28546100$

$-2.27818600$

2.66842500

3.26983700

3.17181300

2.68821600

$-0.32144500$

0.63860900

$-0.99970100$

$-0.74510200$

$-2.42515600$

$-2.42905100$

$-2.52651900$

$-3.31342700$

0.14004400

1. 32514600

1. 24613600

2.15667300

0.04226300

$-1.10569000$

$-2.04824200$

$-1.08593700$

2.68394300

2.63395300

3.15391400

3.35106900

$-0.02910100$

$-0.73026300$

0.95043400

$-0.37561500$

$-2.34800000$

$-3.16402300$

$-2.65089500$

$-2.22421600$

0.28104200

$-0.64123200$

1.12435800

0.40422200

1.39889400

$-0.33757000$
$-1.17854400$

\section{S120}




$\begin{array}{rrrr}\mathrm{H} & -0.49627600 & 0.34588100 & 2.49840900 \\ \mathrm{H} & -0.63800800 & 3.35047800 & 1.75365600 \\ \mathrm{H} & 0.56919400 & 1.72609100 & 3.09701200 \\ \mathrm{C} & -2.50870400 & 2.45203100 & -0.47289400 \\ \mathrm{H} & -3.46006200 & 2.05759200 & -0.09130700 \\ \mathrm{H} & -2.53489500 & 3.54620900 & -0.35855300 \\ \mathrm{H} & -2.41027500 & 2.20365400 & -1.53068700\end{array}$

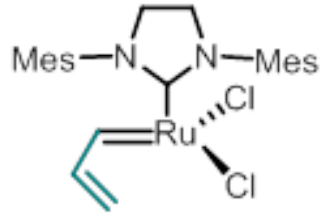

$\mathrm{Ru}$

$\mathrm{Cl}$

CI

$\mathrm{N}$

N

C

C

C

C

C

C

H

C

C

$\mathrm{H}$

$\mathrm{H}$
34

SCF Energy (M06, n-octanol): $\quad$-2056.940529

SCF Energy (B3LYP, gas-phase):

Enthalpy Correction:

Free-Energy Correction:

0.520586

0.420141

\subsection{0 \\ 0.46918900 \\ 1.73369100 \\ $-1.18662300$ \\ 1.00442800 \\ $-1.35784700$ \\ $-2.11146900$ \\ $-1.57113900$ \\ $-0.05034500$ \\ $-2.54979400$ \\ $-3.22293500$ \\ $-4.57587900$ \\ $-5.10294700$ \\ $-5.26451800$}

$-4.56759800$

$-5.09066900$

$-3.21752900$

$-2.51198500$

$-1.97137500$

$-3.22592200$

$-1.78024500$

$-6.71837900$

$-7.21574300$

$-7.26494900$

$-6.82153600$

$-2.51566300$

$-2.17643800$

$-1.63466600$

$-3.19263200$

2. 38251300

2. 89663100

4.24962600

4. 65137700

5.09066300

4.55358900

5.19700600

3.21036200

2. 03986100

1. 21294500

1. 60717600

2.64300100

6.53625000

7.16785000

6.93190200

6.64336300

2.67168100
1.11981800

0.26125400

3.05707400

$-1.42383200$

$-1.52843300$

1.74190700

2. 51883900

3.37136200

$-0.73973700$

$-1.00455500$

$-0.45893300$

$-0.13120600$

0.28885400

$-0.33026500$

$-0.88913600$

$-1.06558400$

$-1.24441700$

$-0.20550000$

$-1.08885100$

0.08528100

0.60493600

0.05821400

0.10209200

$-0.65243700$

1.04730600

$-1.86931800$

$-2.88912100$

$-1.29196200$

$-1.93239500$

$-1.11478300$

$-0.28889500$

0.06267000

0.70839000

$-0.38443300$

$-1.22708600$

$-1.59377500$

$-1.60695500$

0.24391300

$-0.42585500$

1.21726000

0.39588100

0.04842600

$-0.72080200$

0.26832900

0.95978500

$-2.50592000$
$-0.49360500$

$-2.69248100$

$-0.45131400$

0.48459100

0.53309300

$-0.72566300$

0.24740500

1. 14135000

0.21205800

0.32058800

1. 42963700

1.29155200

2.14559400

0.09095000

$-0.98585300$

$-1.92329000$

$-0.89672600$

2.73799500

3.09700200

3.51448400

2.63721100

$-0.04655400$

0.92780800

$-0.67599600$

$-0.51213500$

$-2.07785100$

$-1.85388900$

$-2.37843600$

$-2.93499700$

0.49428300

1.51357100

1.46033100

2.23808700

0.43978200

$-0.54012300$

$-1.33667600$

$-0.53717800$

2. 63989200

2.89366700

2.37345200

3. 54085800

0.37985000

$-0.07729200$

1.37702000

$-0.22265800$

$-1.62316900$

\section{S121}




$\begin{array}{rrrr}\mathrm{H} & 3.46628800 & -2.77396700 & -2.32561700 \\ \mathrm{H} & 1.87577700 & -2.00309100 & -2.18260700 \\ \mathrm{H} & 2.25961600 & -3.44035600 & -1.22080400 \\ \mathrm{C} & -0.91383300 & -2.80693100 & 0.93653100 \\ \mathrm{H} & -1.20532700 & -3.51868800 & 0.15547300 \\ \mathrm{H} & -1.48898400 & -3.03611400 & 1.83820100 \\ \mathrm{C} & 0.60251400 & -2.79191600 & 1.17715700 \\ \mathrm{H} & 0.86166700 & -2.76947300 & 2.24389400 \\ \mathrm{H} & 1.11522800 & -3.64280900 & 0.72096500 \\ \mathrm{H} & -0.50210900 & 3.56534300 & 1.16507900 \\ \mathrm{H} & -1.84606300 & 1.56158700 & -1.69035600 \\ \mathrm{H} & -3.19734900 & 2.41170500 & 0.20399300 \\ \mathrm{H} & -2.19992700 & 3.93876100 & 1.82241300\end{array}$

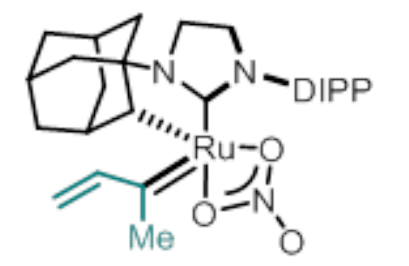

$35 a$

SCF Energy (M06, n-octanol): $\quad-1613.821061$

SCF Energy (B3LYP, gas-phase): $\quad-1613.447044$

Enthalpy Correction: $\quad 0.700402$

Free-Energy Correction: $\quad 0.597143$

$\mathrm{O}$
$\mathrm{O}$
$\mathrm{N}$
$\mathrm{N}$
$\mathrm{C}$
$\mathrm{C}$
$\mathrm{H}$
$\mathrm{H}$
$\mathrm{C}$
$\mathrm{H}$
$\mathrm{H}$
$\mathrm{C}$
$\mathrm{C}$
$\mathrm{H}$
$\mathrm{H}$
$\mathrm{C}$
$\mathrm{H}$
$\mathrm{C}$
$\mathrm{H}$
$\mathrm{H}$
$\mathrm{C}$
$\mathrm{H}$
$\mathrm{C}$
$\mathrm{H}$
$\mathrm{H}$
$\mathrm{C}$
$\mathrm{H}$
$\mathrm{H}$
$\mathrm{C}$
$\mathrm{H}$
$\mathrm{C}$
$\mathrm{H}$
$\mathrm{C}$
$\mathrm{H}$
$\mathrm{H}$
$\mathrm{C}$
$\mathrm{C}$
$\mathrm{C}$
$\mathrm{H}$

-1.37417500
-1.85243300
-0.57780700
1.53172100
0.29525400
0.02029100
-0.05885700
-0.47895600
1.48112800
1.73106800
2.20130600
-2.01238600
-2.72818200
-2.23837800
-2.64574900
-4.21053700
-4.70438400
-4.89470600
-4.86102900
-5.95486600
-4.18390000
-4.66725600
-2.70457300
-2.18676900
-2.65921700
-4.27391200
-3.81154500
-5.32958200
-3.56781000
-3.64192000
-2.09055600
-1.72539100
-4.28413400
-3.83662300
-5.33356900
2.76567500
3.01488100
4.24974100
4.46149200
2.38951000

2.54205400

$-1.54610900$

$-0.94996200$

$-0.66846600$

$-2.46287400$

$-3.50086200$

$-2.39023900$

$-1.96883300$

$-1.50380800$

$-2.77012800$

$-1.42496300$

$-0.62708100$

0.34350800

$-1.17505300$

$-0.41916700$

0.14160300

$-1.78889400$

$-2.35642100$

$-1.65231300$

$-2.57324800$

$-3.55009000$

$-2.80006300$

$-3.39327100$

$-3.36604500$

$-1.76581500$

$-2.32689600$

$-1.61696000$

$-0.39311400$

0.17174500

$-0.69081100$

$-1.40111900$

0.38214800

1.36969700

0.54677300

$-0.27573600$

1.01029600

1.60984300

2.59957000

\begin{abstract}
0.85565500
$-1.25233800$

0.99354500

0.94897600

0.45599500

1.96837800

1.62445800

2.94090000

2.02194300

2.98349000

1.83808300

0.69404900

1.81396100

1.94703700

2.76433400

1. 44796000

2. 25192200

1.27079300

2. 21178000

1. 01807400

0.14902900

0.01547200

0.54469000

$-0.22072200$

1. 48720100

$-1.16567600$

$-1.98907300$

$-1.43298900$

$-0.99177300$

$-1.92865100$

$-0.67259800$

$-1.42518500$

0.13307200

0.27279300

$-0.14741200$

0.65900500

1.19218000

0.90732900

1. 30089800
\end{abstract}

\section{S122}


0.95883300

$-0.31645200$

$-0.82134800$

$-0.95803900$

1.74367200

1.20920800

$-2.36488500$

$-2.64143900$

$-3.38968800$

$-4.40539800$

$-3.18559800$

$-3.36982000$

$-2.44109300$

$-2.21369500$

$-3.45390700$

$-1.74055500$

1.74490700

0.72686600

2. 28797100

2.23272600

3.18137200

3. 62452100

3.82119000

3.19978700

1. 44287600

3.05291300

4.07687800

0.79355000

0.54199700

0.71097700

0.47872000

0.73445100

0.81991800

$-0.52396000$

1.20573900

0.65016800

0.61696200

0.85851700
0.14417600

$-0.35294500$

$-0.94209700$

$-0.11117300$

2.09367300

2.06621300

$-0.66257000$

$-0.46107100$

0.04214500

$-0.30787000$

$-0.17005200$

1.13107600

$-2.18934000$

$-2.47979000$

$-2.54467500$

$-2.70860500$

3.55637200

3.93012300

3. 65525000

4.20548900

1.61590900

2. 21180200

1.71128600

0.56902000

$-0.05904700$

$-0.07699800$

0.13611600

$-0.63137800$

$-2.35769200$

$-2.60166200$

$-3.55431500$

$-3.80921900$

$-1.72058000$

$-4.00595700$

$-4.33194700$

$-3.27072000$

$-4.74011700$

$-3.89383100$

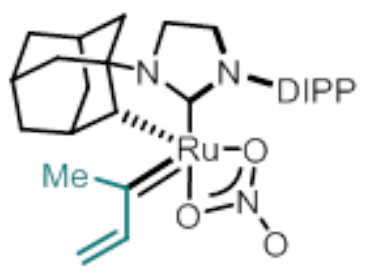

$35 b$

SCF Energy (M06, n-octanol):

$-1613.824309$

SCF Energy (B3LYP, gas-phase):

$-1613.449028$

Enthalpy Correction:

0.701063

Free-Energy Correction:

0.600762

$\mathrm{O}$
$\mathrm{O}$
$\mathrm{N}$
$\mathrm{N}$
$\mathrm{C}$
$\mathrm{C}$
$\mathrm{H}$
$\mathrm{H}$
$\mathrm{C}$
$\mathrm{H}$
$\mathrm{H}$
$\mathrm{C}$

-1.01794700
-1.25833800
-0.65191400
1.45105000
0.23488500
-0.09122600
-0.34911100
-0.46150900
1.42053900
1.92960700
1.92420700
-2.07012500

1.13816700

$-2.23275200$

$-0.91021700$

$-2.92781000$

0.35548200

1.86834000

0.59595600

1.25253300

0.13658700

0.85808700

1.20828300

2.92364100

0.83869500

3.92024600

2.23837200

2. 83310700

1.11700800

2.63481400

2.08244700

2. 69867000

0.41761300

3. 31383300

0.33000000

1.47127600

\section{S123}




\begin{tabular}{|c|c|c|}
\hline 50721900 & 1.71030000 & 0.91495000 \\
\hline-1.83 & 2.01065500 & 0.10699500 \\
\hline 2.44 & 2.47063800 & 1.70666700 \\
\hline 39 & 1.63275800 & 0.38725300 \\
\hline 4.24981000 & 2.61853500 & 0.0056140 \\
\hline 4.89560500 & 1.21442700 & 1.533074 \\
\hline 4.87290900 & 1.96328800 & 2.337841 \\
\hline 5.93173300 & 1.16496400 & 1.1718 \\
\hline 4.46 & -0.163045 & 2.073 \\
\hline & -0.4715 & 2.8868 \\
\hline-3.01720900 & -0.06176900 & 2.626442 \\
\hline 2 . & -1.020564 & \\
\hline 82892 & 0.68601400 & 3.4331 \\
\hline 9129 & -1.196300 & 0.9 \\
\hline 4. & -2.196049 & 1.29 \\
\hline 5. & -1.2569 & 0.5 \\
\hline-3 . & -0.7 & -0.22 \\
\hline 3. & -1 & -1.0 \\
\hline-2 . & -0.7690 & 0.3871 \\
\hline-2.02816 & -1.72299000 & 0.908 \\
\hline 4. & 0.5 & -0.7 \\
\hline-3 . & 0. & -1.5 \\
\hline-5 & 0. & $-1 \cdot 1$ \\
\hline 2.63086 & 0.6836 & $0.432 \varepsilon$ \\
\hline 2.812 & 1.829 & -0.3788 \\
\hline 3.97 & 1.90823600 & -1.15486 \\
\hline 4.133518 & 2.77 & -1.7895 \\
\hline 4 . & 0. & -1.11 \\
\hline 4. & -0 & -0 \\
\hline 5.52 & -0 . & -0.2 \\
\hline 3.611 & -0 . & 0.5020 \\
\hline 1.8382 & 3.00633 & -0.3822610 \\
\hline 0.933642 & 50700 & 0.15156 \\
\hline 3.4 & -1.5 & 1.4 \\
\hline 2 & -1 & 1.8 \\
\hline 4 . & -1 & 2.6767 \\
\hline 4.2733 & -2.16430700 & 3.384846 \\
\hline 5.4592 & -1.31457300 & 2.3782 \\
\hline 4.193168 & -0.40046400 & 3.2049990 \\
\hline 3. & -2.866799 & 0.7 \\
\hline 4 . & -2 . & 0.4 \\
\hline 3. & -3 . & 1.4 \\
\hline 3.1646 & -3.01353100 & -0.1276360 \\
\hline 2.4457 & 4.20604100 & 0.3778870 \\
\hline 2.735391 & 3.93930100 & 1.40129700 \\
\hline 3.342844 & 4.57983100 & -0.1299340 \\
\hline 1 . & 5. & 0 . \\
\hline 1. & 3. & -1.7984 \\
\hline 0.68 & 4.25257300 & -1.7348420 \\
\hline 2.257276 & 3.78914700 & -2.3915660 \\
\hline 0.926022 & 2.60918300 & -2.33413100 \\
\hline 5.832321 & 0.98541000 & -1.73093500 \\
\hline 1338645 & 0.34518700 & -3.2011590 \\
\hline-1.69906 & 0.75498300 & -4.2859610 \\
\hline-0.437644 & -0.62584600 & -0.8551720 \\
\hline 0.066973 & -2.39307700 & -0.4118630 \\
\hline 0.416544 & -3.20357300 & -1.5856810 \\
\hline 5473 & -3.09858700 & 0.9214480 \\
\hline 0.797479 & -4.49811600 & -1.5808080 \\
\hline 0.3435210 & -2.70359200 & -2.5510930 \\
\hline 1.024413 & -3.64226600 & 1.0838950 \\
\hline-0.717673 & -3.85037600 & 0.9533280 \\
\hline $.06 / 1 / 36$ & - & 1 \\
\hline
\end{tabular}

\section{S124}




$\begin{array}{llll}\mathrm{H} & 0.88482100 & -5.08196300 & -0.66905400 \\ \mathrm{H} & 1.02860700 & -5.01645200 & -2.50752400\end{array}$

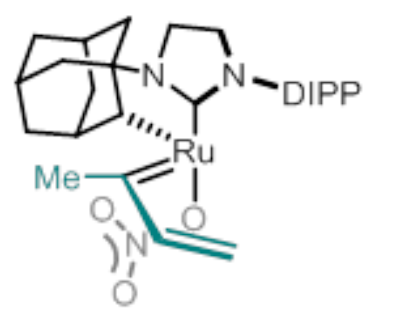

$35 \mathrm{c}$

SCF Energy (M06, n-octanol): $\quad-1613.818753$

SCF Energy (B3LYP, gas-phase): $\quad-1613.430501$

Enthalpy Correction: $\quad 0.699911$

Free-Energy Correction: $\quad 0.595317$

3.35684800

2.23699300

$-1.95546200$

$-1.36773900$

$-0.90536300$

$-3.25287500$

$-3.87579500$

$-3.80097200$

$-2.82837400$

$-3.30714300$

$-3.04099000$

$-1.73141500$

$-1.92933500$

$-1.27969700$

$-2.96594200$

$-1.58771900$

$-1.74427300$

$-2.50562400$

$-3.55853000$

$-2.27625000$

$-2.29477400$

$-2.94358800$

$-2.66916200$

$-2.56354600$

$-3.72225800$

$-0.81618700$

$-0.65618300$

$-0.56372500$

0.10458900

1.14921400

$-0.26464100$

$-0.23033900$

$-0.11313200$

0.54747300

0.14820500

$-0.62526800$

$-0.41529300$

0.27192400

0.45621900

0.71327200

0.47840800

0.82507600

$-0.18859300$

$-0.91697000$

$-1.45375000$

$-0.42968200$

$-0.80065200$

$-1.50111200$

$-1.70175500$
0.49892800

$-1.24214700$

0.34726600

0.12647700

0.14486100

0.31168600

1.16520900

$-0.60636500$

0.35483100

$-0.42100400$

1. 32596000

0.28147800

$-1.16622500$

$-1.84847600$

$-1.49048100$

$-1.23868800$

$-2.26569700$

$-0.27396300$

$-0.56918100$

$-0.32434600$

1.16472200

1.85515900

1.22552700

2. 24901800

0.93236200

1. 56445800

2.59924300

1. 53046500

0.59984500

0.89551200

0.73480400

1.80134500

$-0.83405000$

$-1.53711700$

$-0.87861600$

$-0.16713000$

$-1.51859400$

$-1.78203300$

$-2.81208600$

$-0.74851900$

0.57523100

1.37457000

0.89504700

$-2.68621300$

$-2.27460000$

2. 35815300

2. 39022500

2. 98845100

4.02399800

\section{S125}




$\begin{array}{lrrr}\mathrm{H} & -5.28081300 & -1.16672100 & 3.00138300 \\ \mathrm{H} & -4.20187000 & -2.44632800 & 2.43484500 \\ \mathrm{C} & -3.36576900 & 0.86673000 & 3.19024300 \\ \mathrm{H} & -4.38882700 & 1.24699900 & 3.29342000 \\ \mathrm{H} & -2.98354000 & 0.67912100 & 4.20047000 \\ \mathrm{H} & -2.76071900 & 1.65397100 & 2.73133500 \\ \mathrm{C} & -3.15677600 & -1.91249900 & -3.56430600 \\ \mathrm{H} & -3.54081200 & -2.75289600 & -2.97428600 \\ \mathrm{H} & -4.01284000 & -1.43362200 & -4.05355000 \\ \mathrm{H} & -2.50974700 & -2.31666500 & -4.35163300 \\ \mathrm{C} & -1.81508100 & 0.24589400 & -3.53036100 \\ \mathrm{H} & -1.18754600 & -0.13939700 & -4.34259400 \\ \mathrm{H} & -2.62056100 & 0.83631500 & -3.98266700 \\ \mathrm{H} & -1.20318400 & 0.92059900 & -2.92107900 \\ \mathrm{H} & -6.15513000 & 1.23918000 & -0.97484100 \\ \mathrm{~N} & 2.60304000 & 3.13230400 & -0.71873700 \\ \mathrm{O} & 3.36641000 & 3.69423400 & -1.49627000 \\ \mathrm{Ru} & 0.58828800 & 0.88577200 & -0.20657600 \\ \mathrm{C} & -0.06569400 & 1.88290000 & 1.19814000 \\ \mathrm{C} & -0.89152700 & 3.02200700 & 0.76298200 \\ \mathrm{C} & 0.39234000 & 1.89422200 & 2.63282600 \\ \mathrm{C} & -1.92628600 & 2.98115900 & -0.09389600 \\ \mathrm{H} & -0.58527400 & 3.98846200 & 1.17337500 \\ \mathrm{H} & -0.29904500 & 2.44628800 & 3.28236900 \\ \mathrm{H} & 1.36808300 & 2.39846800 & 2.66861600 \\ \mathrm{H} & 0.52346700 & 0.88611700 & 3.03301900 \\ \mathrm{H} & -2.30416500 & 2.04707400 & -0.49557000 \\ \mathrm{H} & -2.43865200 & 3.89144500 & -0.39334200\end{array}$

\section{Alkenes}

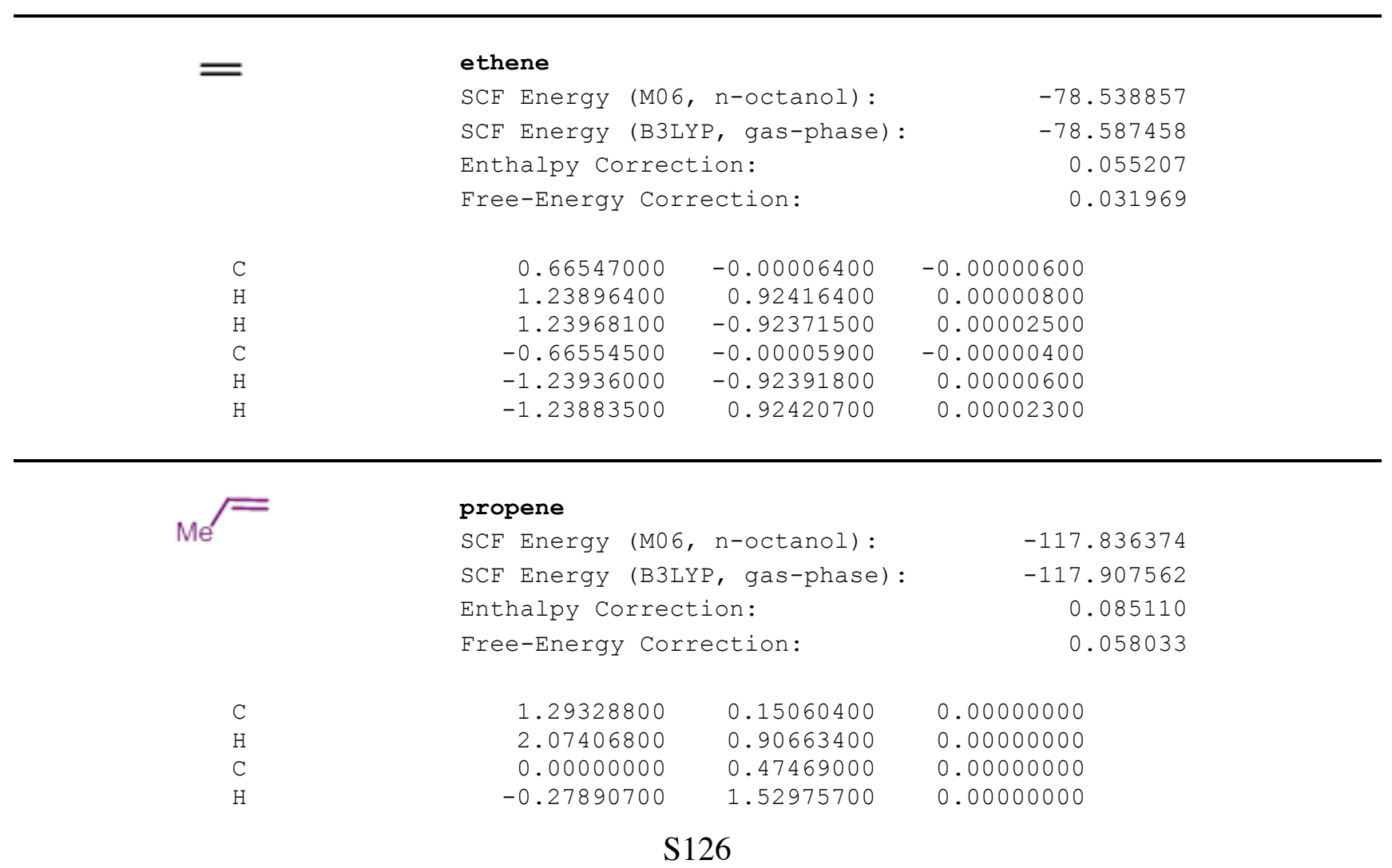




$\begin{array}{lrrr}\mathrm{C} & -1.13904800 & -0.50467900 & 0.00000000 \\ \mathrm{H} & -0.77952200 & -1.53936200 & 0.00000000 \\ \mathrm{H} & -1.78145800 & -0.36674800 & 0.88045800 \\ \mathrm{H} & -1.78145800 & -0.36674800 & -0.88045800 \\ \mathrm{H} & 1.62184100 & -0.88722600 & 0.00000000\end{array}$

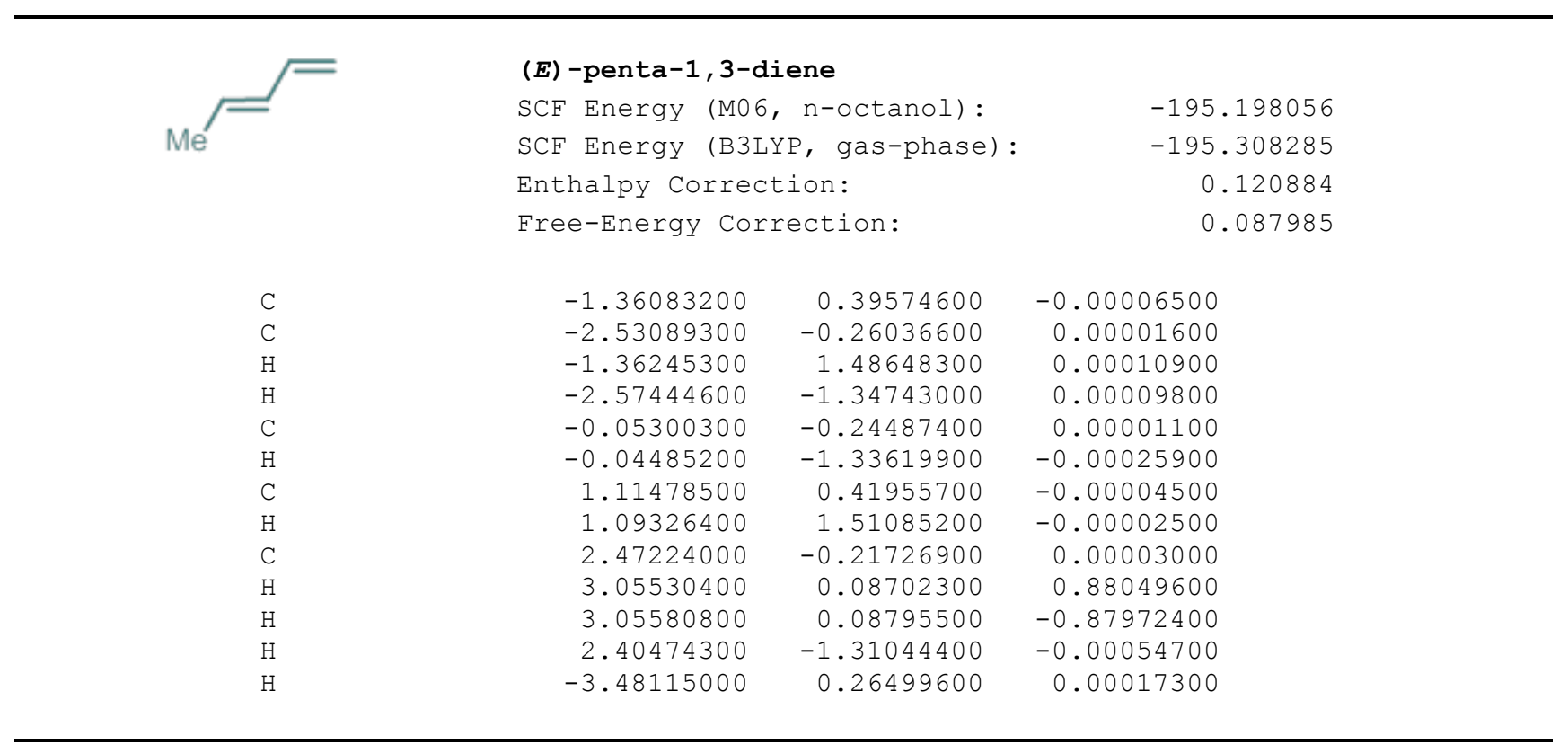

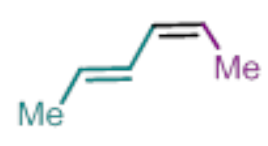

$\mathrm{C}$
$\mathrm{C}$
$\mathrm{H}$
$\mathrm{H}$
$\mathrm{C}$
$\mathrm{H}$
$\mathrm{H}$
$\mathrm{H}$
$\mathrm{C}$
$\mathrm{H}$
$\mathrm{C}$
$\mathrm{H}$
$\mathrm{C}$
$\mathrm{H}$
$\mathrm{H}$
$\mathrm{H}$

\section{$(2 Z, 4 E)$-hexa-2 , 4-diene}

SCF Energy (M06, n-octanol): $\quad-234.493331$

SCF Energy (B3LYP, gas-phase):

Enthalpy Correction:

Free-Energy Correction:

$$
\begin{array}{r}
-234.493331 \\
-234.625085 \\
0.150894 \\
0.114007
\end{array}
$$

$$
\begin{array}{r}
-0.03242600 \\
0.39157100 \\
-1.10533600 \\
1.46604700 \\
-0.49831700 \\
-0.31419800 \\
-0.31461400 \\
-1.55742700 \\
0.86203700 \\
1.92559500 \\
0.51853500 \\
1.32770600 \\
-0.86486500 \\
-1.01834700 \\
-1.01811600 \\
-1.65051700
\end{array}
$$

$$
\begin{array}{r}
-0.00007600 \\
-0.00006600 \\
0.00002100 \\
0.00008900 \\
0.00004400 \\
0.88000000 \\
-0.88020500 \\
0.00033100 \\
0.00003900 \\
0.00002300 \\
-0.00002800 \\
0.00010100 \\
-0.00000600 \\
0.88033100 \\
-0.87962000 \\
-0.00051500
\end{array}
$$

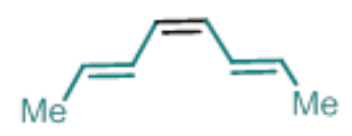

$(2 E, 4 Z, 6 E)$-octa-2, 4, 6-triene

$\mathrm{SCF}$ Energy (M06, n-octanol): SCF Energy (B3LYP, gas-phase): Enthalpy Correction:

Free-Energy Correction:

$$
\begin{array}{r}
-311.856289 \\
-312.029564 \\
0.186578 \\
0.143970
\end{array}
$$

\section{S127}




$\begin{array}{lrrr}\text { C } & -0.67830200 & 1.19048200 & 0.00001500 \\ \mathrm{C} & 0.67830100 & 1.19047600 & -0.00000300 \\ \mathrm{H} & -1.18004800 & 2.15795400 & -0.00008300 \\ \mathrm{H} & 1.18005600 & 2.15794300 & 0.00010000 \\ \mathrm{C} & 1.55645000 & 0.03531300 & 0.00001200 \\ \mathrm{H} & 1.09936800 & -0.95379200 & -0.00006300 \\ \mathrm{C} & 2.90023300 & 0.11823600 & 0.00005700 \\ \mathrm{H} & 3.36087900 & 1.10778600 & -0.00003000 \\ \mathrm{C} & 3.83868400 & -1.05067200 & -0.00002700 \\ \mathrm{H} & 4.49736700 & -1.03449300 & 0.87982600 \\ \mathrm{H} & 4.49722900 & -1.03458500 & -0.87999600 \\ \mathrm{H} & 3.29754200 & -2.00300300 & 0.00006300 \\ \mathrm{C} & -1.55645300 & 0.03532100 & -0.00002300 \\ \mathrm{H} & -1.09936600 & -0.95378200 & 0.00005200 \\ \mathrm{C} & -2.90023600 & 0.11823700 & -0.00007100 \\ \mathrm{H} & -3.36088900 & 1.10778500 & 0.00000700 \\ \mathrm{C} & -3.83868000 & -1.05067700 & 0.00003500 \\ \mathrm{H} & -4.49718300 & -1.03462100 & 0.88003800 \\ \mathrm{H} & -4.49740400 & -1.03447700 & -0.87978400 \\ \mathrm{H} & -3.29753000 & -2.00300400 & -0.00010600\end{array}$
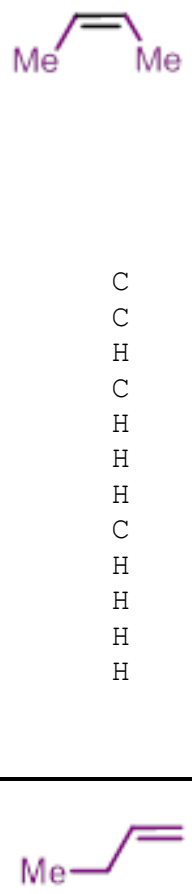

$\mathrm{C}$
$\mathrm{H}$
$\mathrm{C}$
$\mathrm{H}$
$\mathrm{C}$
$\mathrm{H}$
$\mathrm{H}$
$\mathrm{H}$
$\mathrm{C}$
$\mathrm{H}$

\section{( $z$ ) -but-2-ene}

SCF Energy (M06, n-octanol): SCF Energy (B3LYP, gas-phase): Enthalpy Correction:

Free-Energy Correction:

$$
\begin{array}{r}
-0.66950300 \\
0.66914700 \\
1.16974300 \\
1.59339500 \\
1.06097700 \\
2.25092000 \\
2.25062600 \\
-1.59321200 \\
-2.25190800 \\
-1.05993700 \\
-2.24949100 \\
-1.16989600
\end{array}
$$

$$
\begin{array}{r}
-157.131025 \\
-157.221726 \\
0.115112 \\
0.083868
\end{array}
$$
$-0.00004200$
0.00000300
0.00011000
$-0.00004600$
$-0.00019500$
$-0.88010900$
0.88023400
0.00003200
$-0.87921000$
$-0.00172800$
0.88115500
0.00006800

\section{1-butene}

SCF Energy (M06, n-octanol): SCF Energy (B3LYP, gas-phase): Enthalpy Correction:

Free-Energy Correction:

$$
\begin{array}{r}
-157.2180532 \\
-157.1272270 \\
0.115231 \\
0.084472
\end{array}
$$

-1.86133200
-2.74072200
-0.72357600
-0.67346300
0.54047400
0.36659400
0.80195100
-1.95808400
1.72883800
2.64085400

0.01723000

$-0.61897300$

$-0.29513200$

$-1.22033900$

0.52134100

1.44442900

0.82775700

0.92699400

$-0.24733700$

0.36016900

\section{S128}

$$
\begin{array}{r}
-0.28037400 \\
-0.22307800 \\
0.34170700 \\
0.91981200 \\
0.30814900 \\
-0.25936500 \\
1.33225900 \\
-0.87023900 \\
-0.29500900 \\
-0.27626000
\end{array}
$$




$\begin{array}{rrrr}\mathrm{H} & 1.92991100 & -1.16895900 & 0.26475600 \\ \mathrm{H} & 1.52654000 & -0.52769000 & -1.33472200\end{array}$

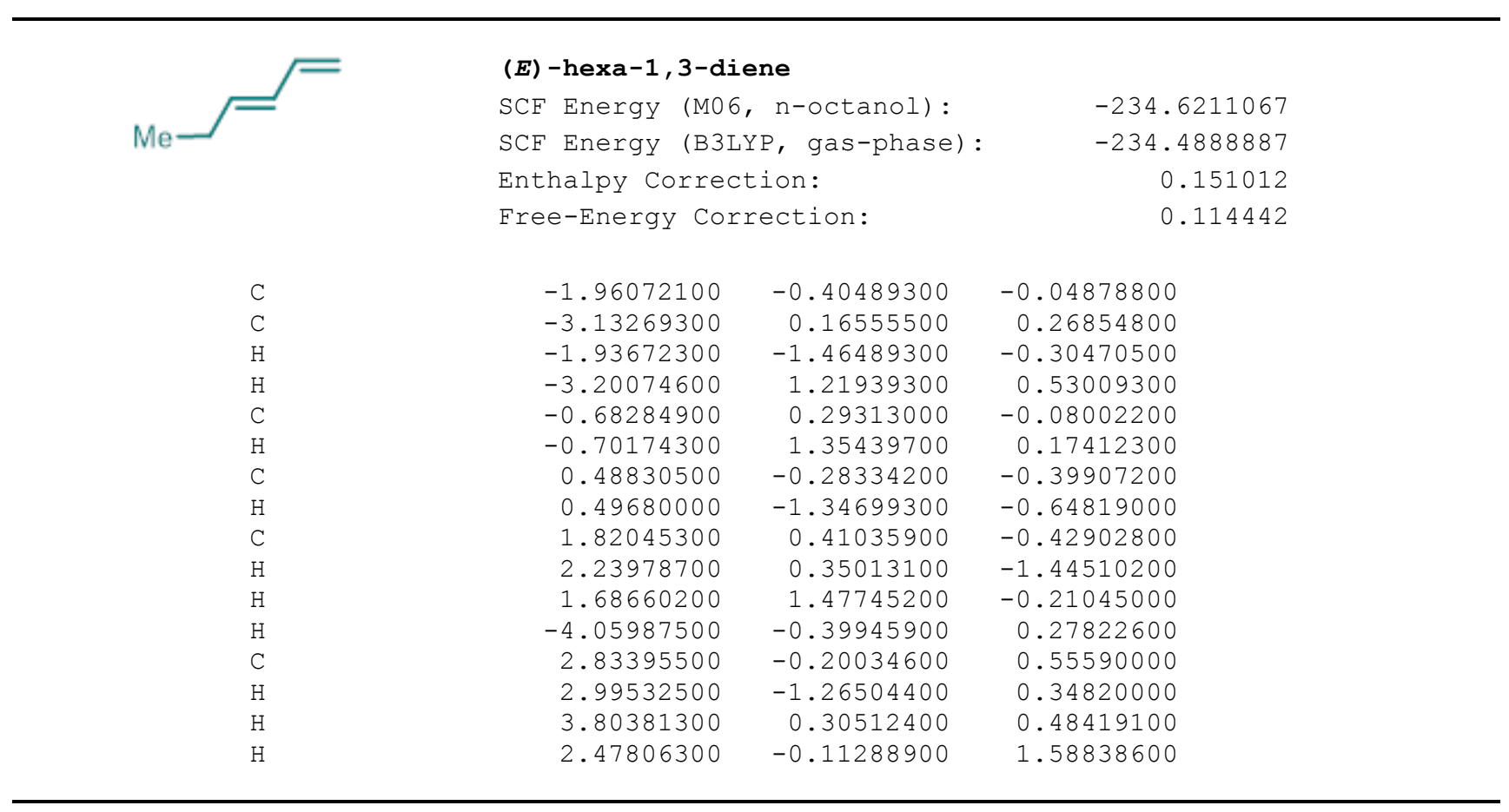




\section{References}

(1) Johns, A. M.; Ahmed, T. S.; Jackson, B. W.; Grubbs, R. H.; Pederson, R. L. Org. Lett. 2016, 18 , $772-775$.

(2) Aponick, A.; Li, C.-Y.; Biannic B. Org. Lett. 2008, 10, 669-671.

(3) Hodgson, D. M.; Fleming, M. J.; Stanway, S. J. J. Org. Chem. 2007, 72, 4763-4773.

(4) Lebel, H.; Davi, M.; Díez-González, S.; Nolan S. P. J. Org. Chem. 2007, 72, 144-149.

(5) Gaussian 09, Revision D.01, M. J. Frisch, G. W. Trucks, H. B. Schlegel, G. E. Scuseria, M. A. Robb, J. R. Cheeseman, G. Scalmani, V. Barone, B. Mennucci, G. A. Petersson, H. Nakatsuji, M. Caricato, X. Li, H. P. Hratchian, A. F. Izmaylov, J. Bloino, G. Zheng, J. L. Sonnenberg, M. Hada, M. Ehara, K. Toyota, R. Fukuda, J. Hasegawa, M. Ishida, T. Nakajima, Y. Honda, O. Kitao, H. Nakai, T. Vreven, J. A. Montgomery, Jr., J. E. Peralta, F. Ogliaro, M. Bearpark, J. J. Heyd, E. Brothers, K. N. Kudin, V. N. Staroverov, T. Keith, R. Kobayashi, J. Normand, K. Raghavachari, A. Rendell, J. C. Burant, S. S. Iyengar, J. Tomasi, M. Cossi, N. Rega, J. M. Millam, M. Klene, J. E. Knox, J. B. Cross, V. Bakken, C. Adamo, J. Jaramillo, R. Gomperts, R. E. Stratmann, O. Yazyev, A. J. Austin, R. Cammi, C. Pomelli, J. W. Ochterski, R. L. Martin, K. Morokuma, V. G. Zakrzewski, G. A. Voth, P. Salvador, J. J. Dannenberg, S. Dapprich, A. D. Daniels, O. Farkas, J. B. Foresman, J. V. Ortiz, J. Cioslowski, and D. J. Fox, Gaussian, Inc., Wallingford CT, 2010.

(6) Legault, C. Y. CYLView, 1.0b; Université de Sherbrooke, Canada, 2009; http://www.cylview.org.

(7) (a) Becke, A. D. J. Chem. Phys. 1993, 98, 5648-5652. (b) Lee, C.; Yang, W.; Parr, R. G. Phys. Rev. B 1988, 37, 785-789.

(8) (a) Zhao,Y.; Truhlar, D. G. Theor. Chem. Acc. 2008, 120, 215-241. (b) Zhao, Y.; Truhlar, D. G. Acc. Chem. Res. 2008, 41, 157-167.

(9) Marenich, A. V.; Cramer, C. J.; Truhlar, D. G. J. Phys. Chem. B 2009, 113, 6378-6396.

(10) Trnka, T. M.; Day, M. W.; Grubbs, R. H. Organometallics 2001, 20, 3845-3845. 
${ }^{1} \mathrm{H}$ NMR $\left(400 \mathrm{MHz}, \mathrm{CDCl}_{3}\right.$ ) spectrum of compound 8c.

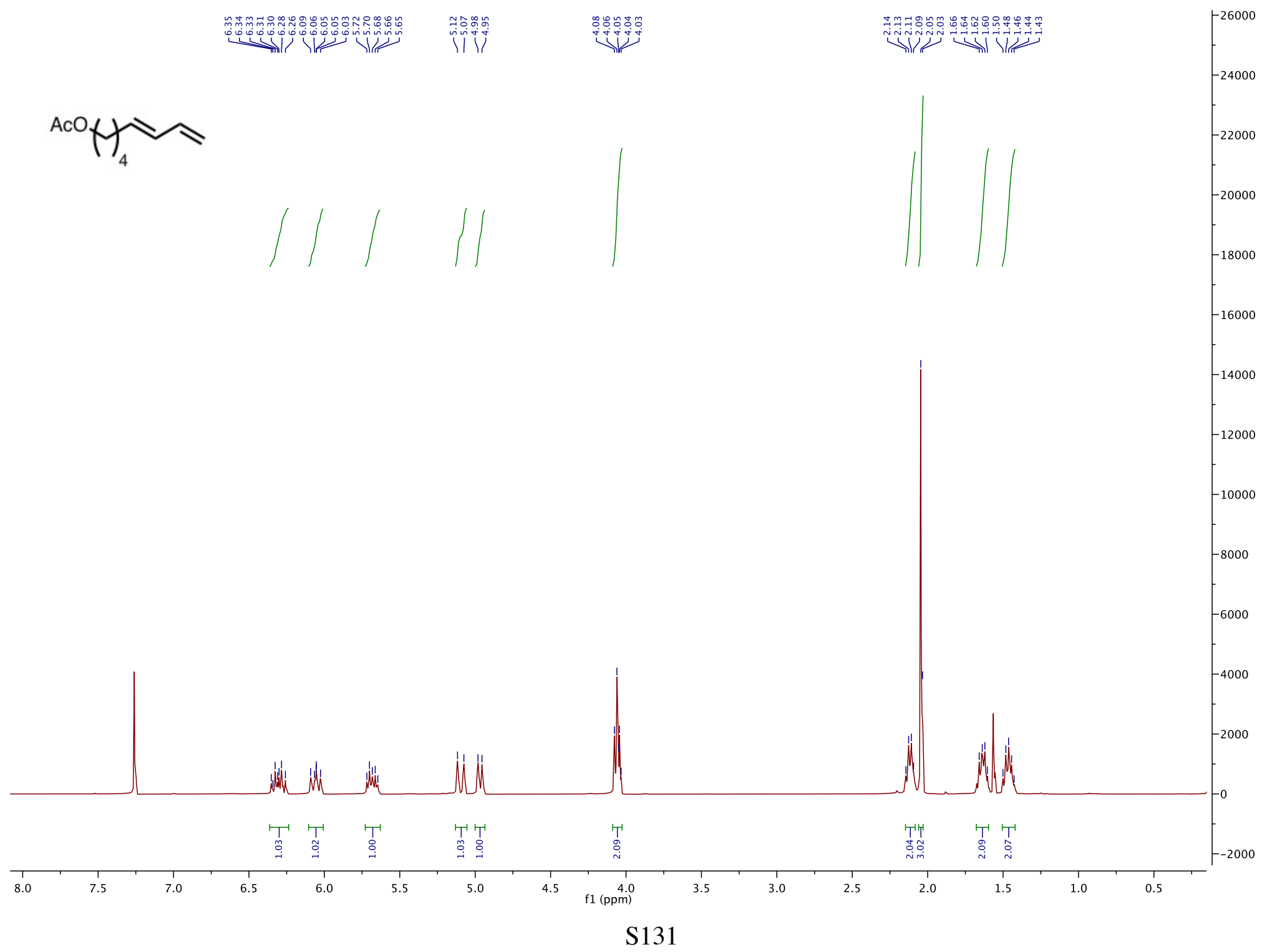


${ }^{13} \mathrm{C}$ NMR (101 MHz, $\mathrm{CDCl}_{3}$ ) spectrum of compound 8c.

$\stackrel{\substack{i \\ \hat{i}}}{\hat{i}}$<smiles>C=CC=CC(C)C(C)OC(C)=O</smiles>
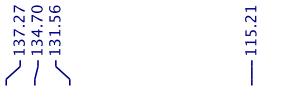

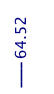

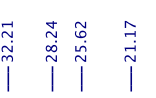

$-6000$

5500

5000

4500

$-3500$

$-3000$

2500

2000

1500

1000

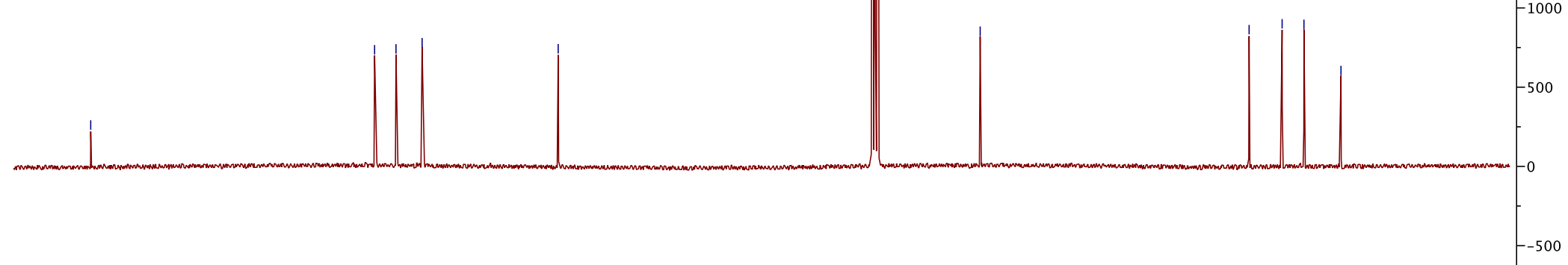

$-500$

$160 \quad 150$

$140 \quad 130$

$120 \quad 110$

100 
${ }^{1} \mathrm{H}$ NMR $\left(400 \mathrm{MHz}, \mathrm{CDCl}_{3}\right.$ ) spectrum of compound 8e.

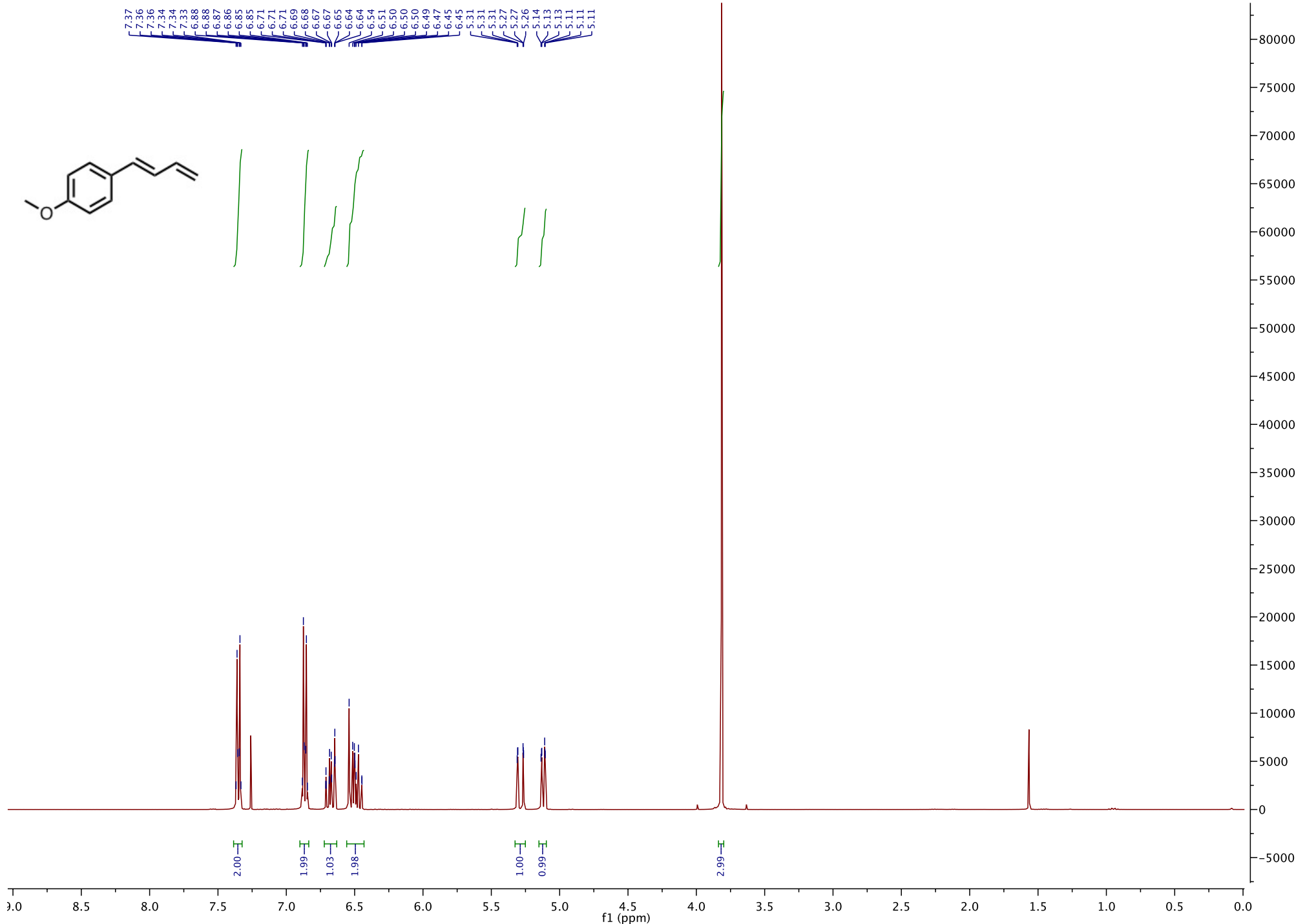

S133 
${ }^{13} \mathrm{C}$ NMR (101 MHz, $\mathrm{CDCl}_{3}$ ) spectrum of compound 8e.

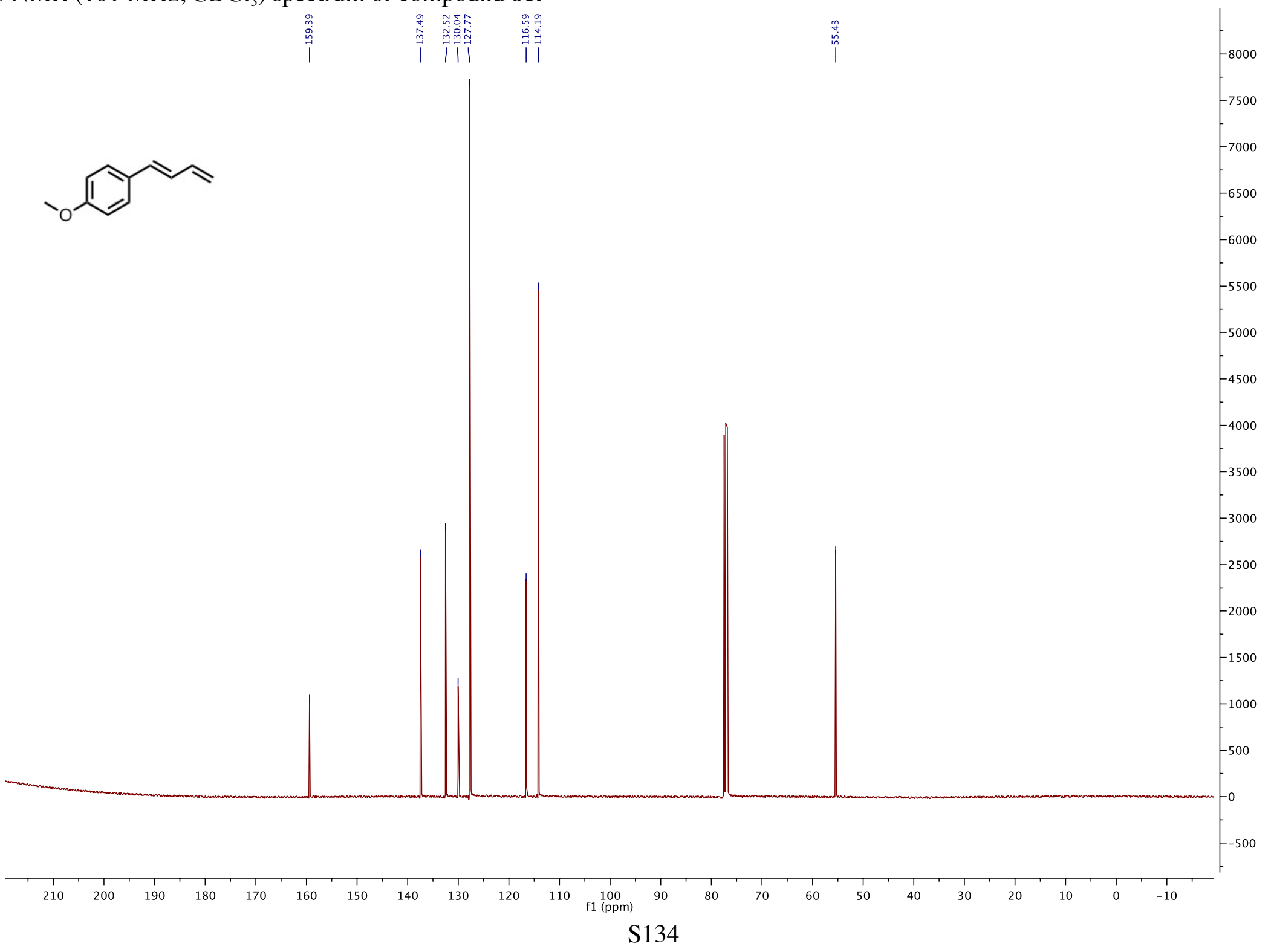


${ }^{1} \mathrm{H}$ NMR (400 MHz, $\mathrm{CDCl}_{3}$ ) spectrum of compound $8 \mathbf{8 f}$.

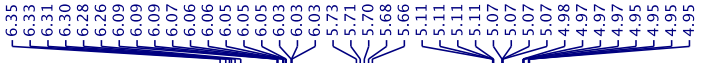

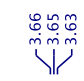

$\mathrm{HOH}_{4} \mathrm{~N}$

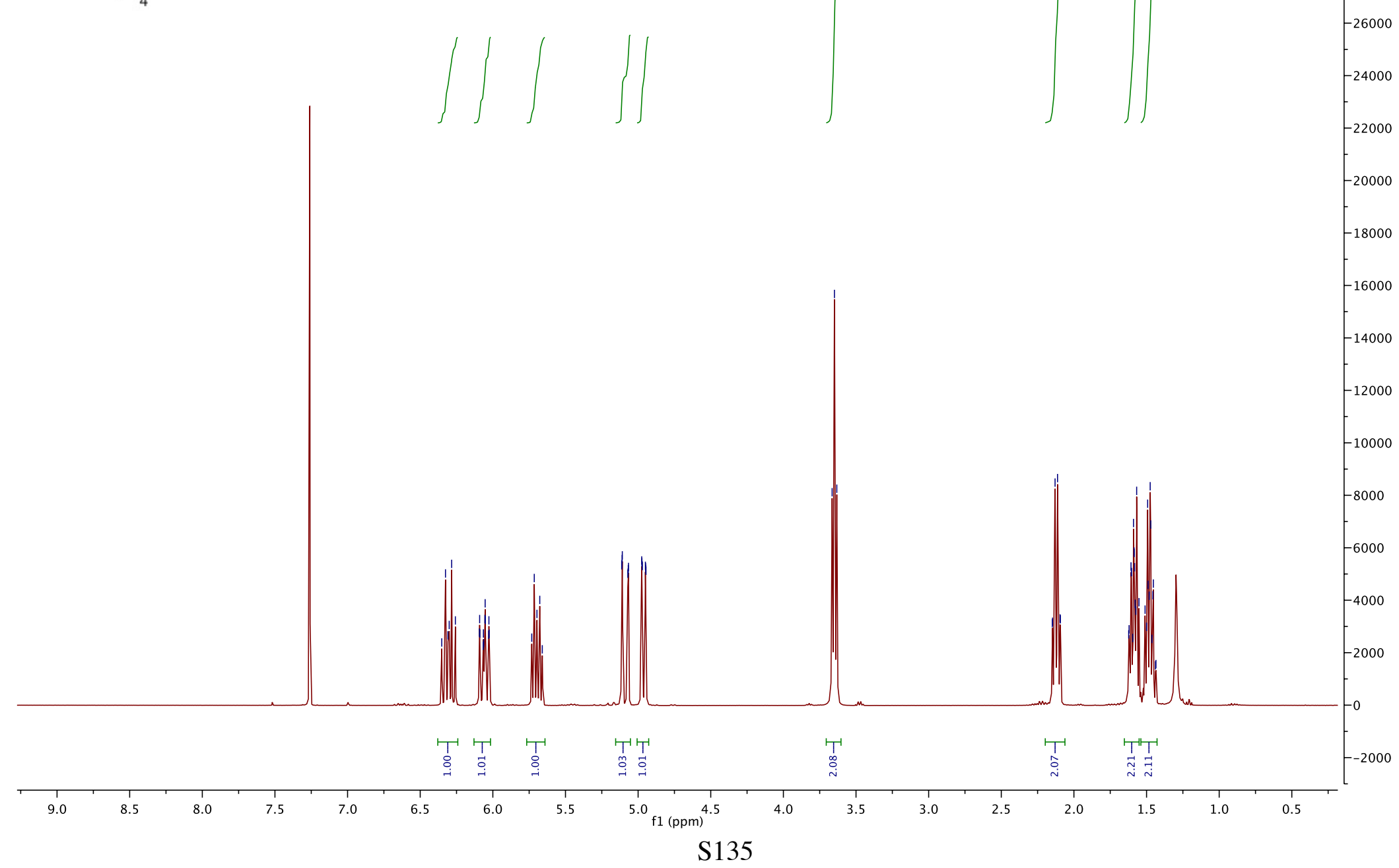


${ }^{13} \mathrm{C}$ NMR (101 MHz, $\mathrm{CDCl}_{3}$ ) spectrum of compound $\mathbf{8 f}$.

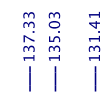

$\stackrel{\hat{\dot{\theta}}}{\vec{i}}$

$\mathrm{HO}_{4} \mathrm{~N}$

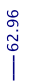

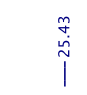

5000

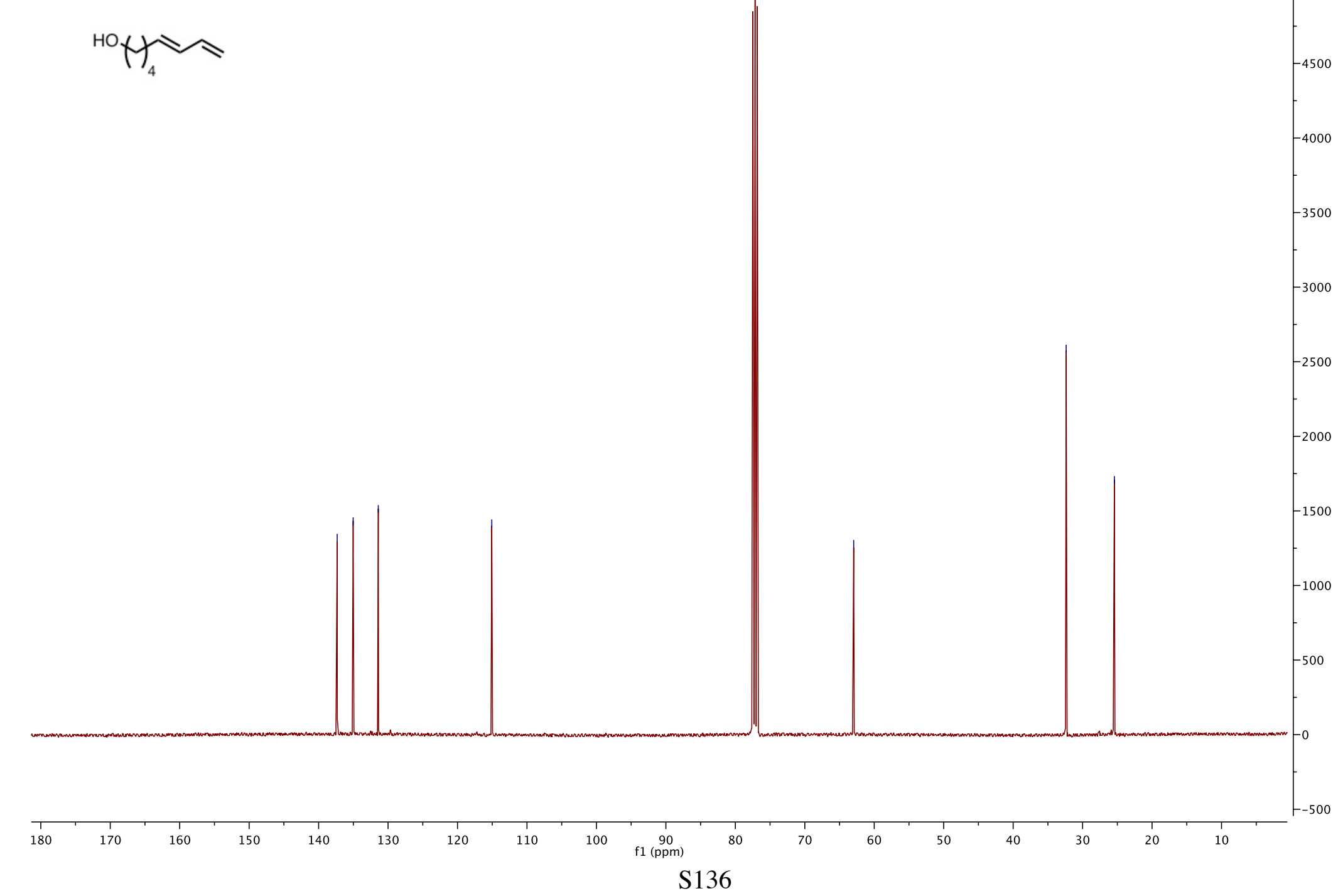


${ }^{1} \mathrm{H}$ NMR (500 MHz, $\mathrm{CDCl}_{3}$ ) spectrum of compound 9a.

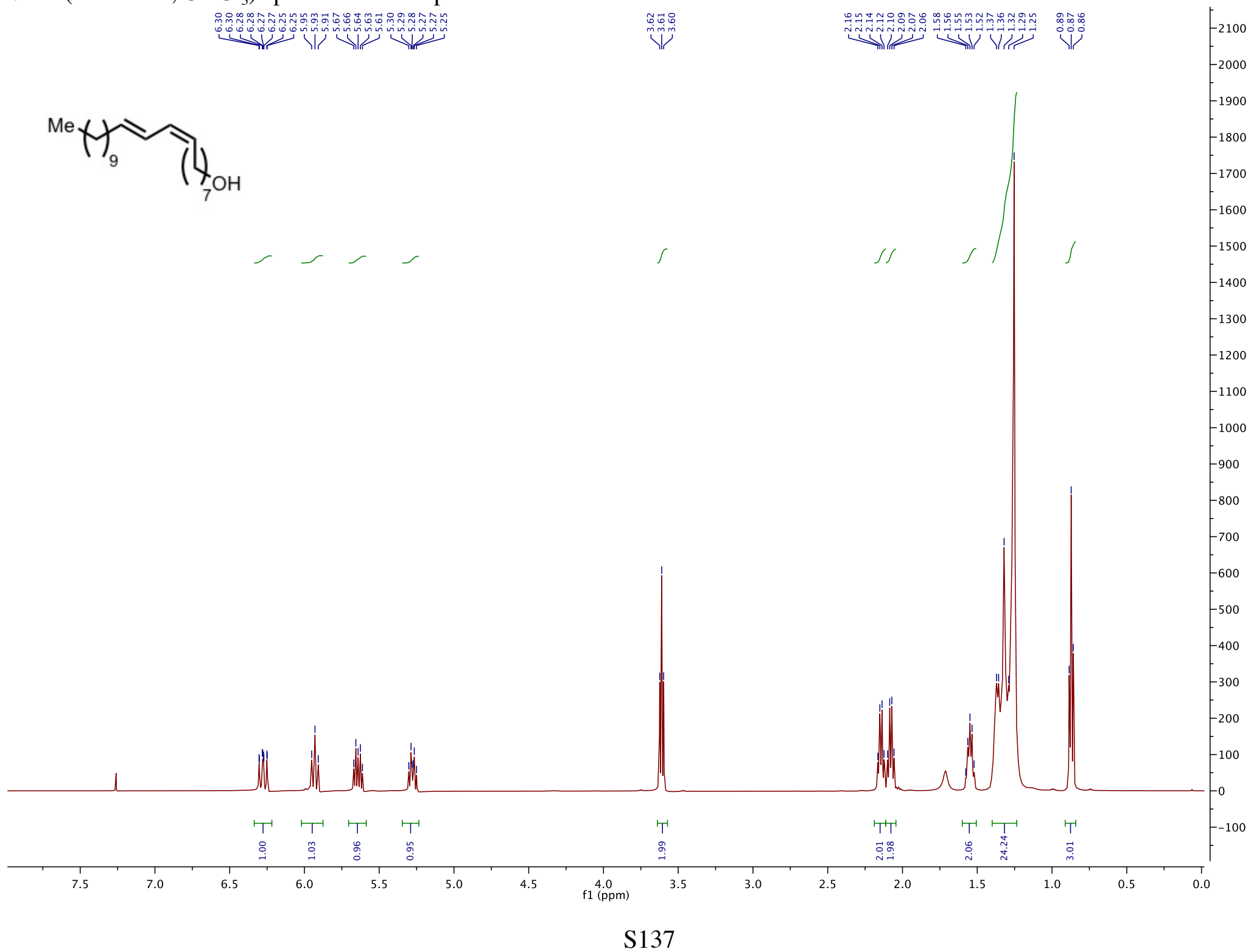


${ }^{13} \mathrm{C}$ NMR (126 MHz, $\mathrm{CDCl}_{3}$ ) spectrum of compound 9a.

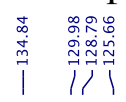

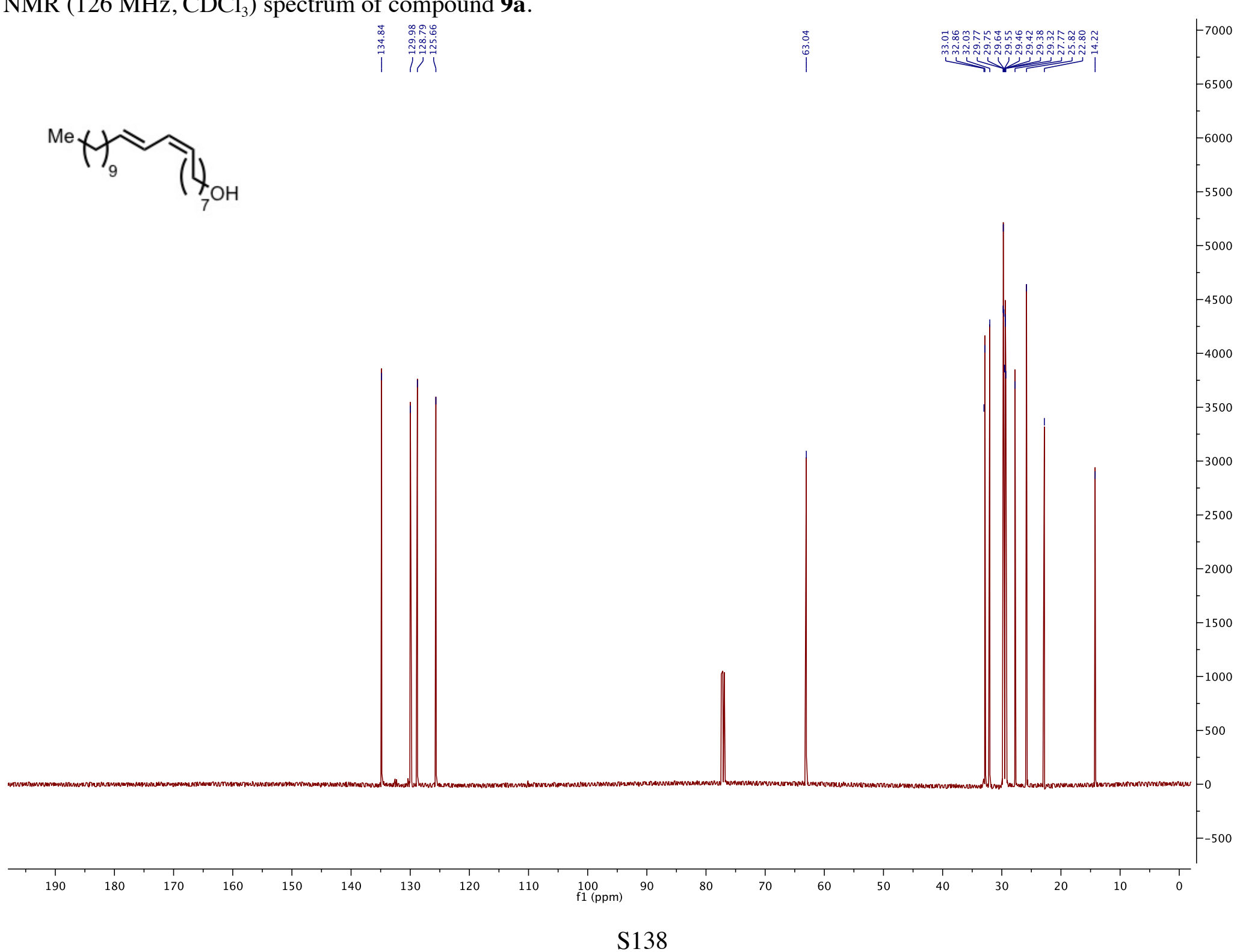


gCOSY spectrum of compound 9a.

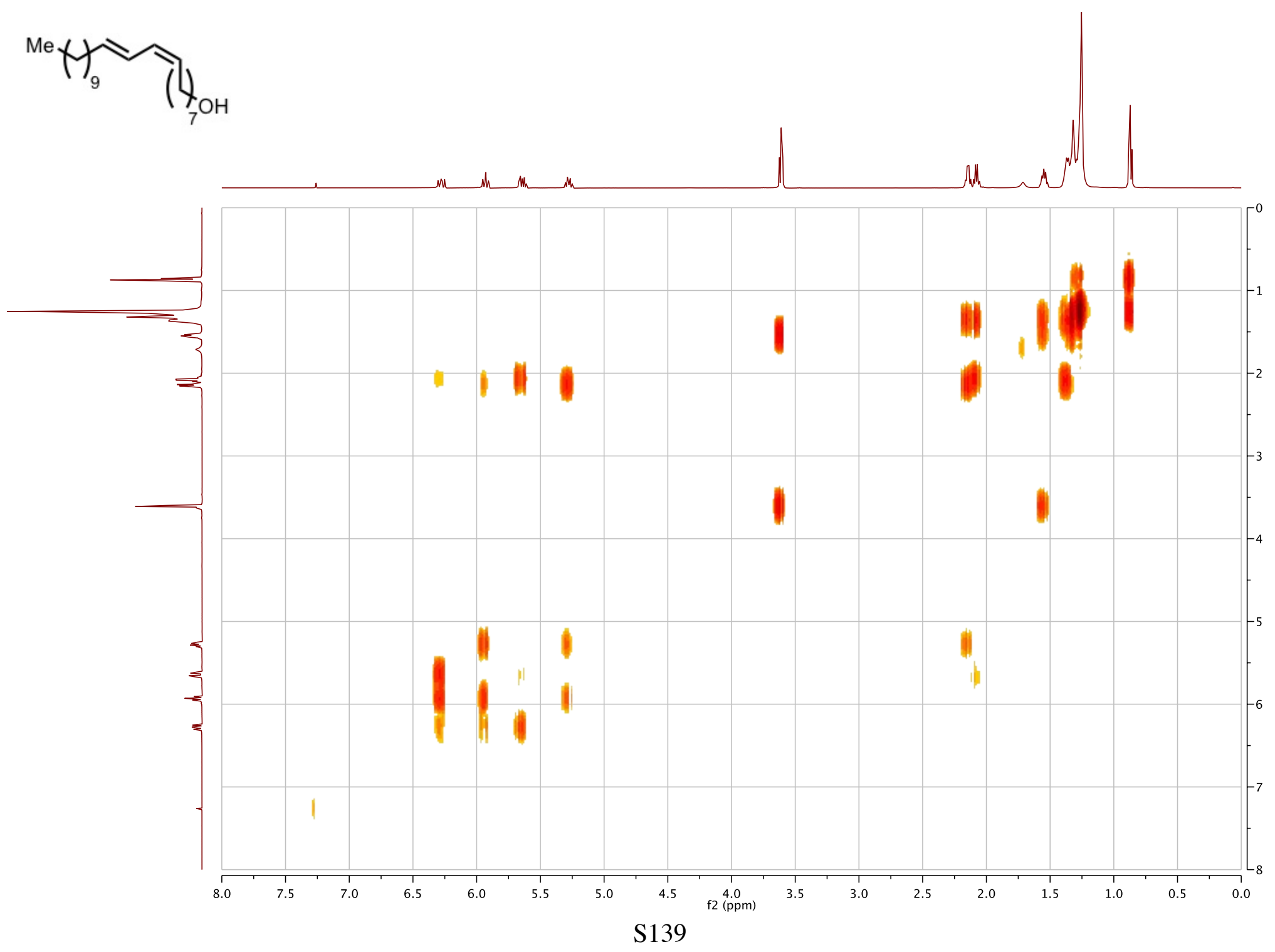


HSQC spectrum of compound $\mathbf{9 a}$.

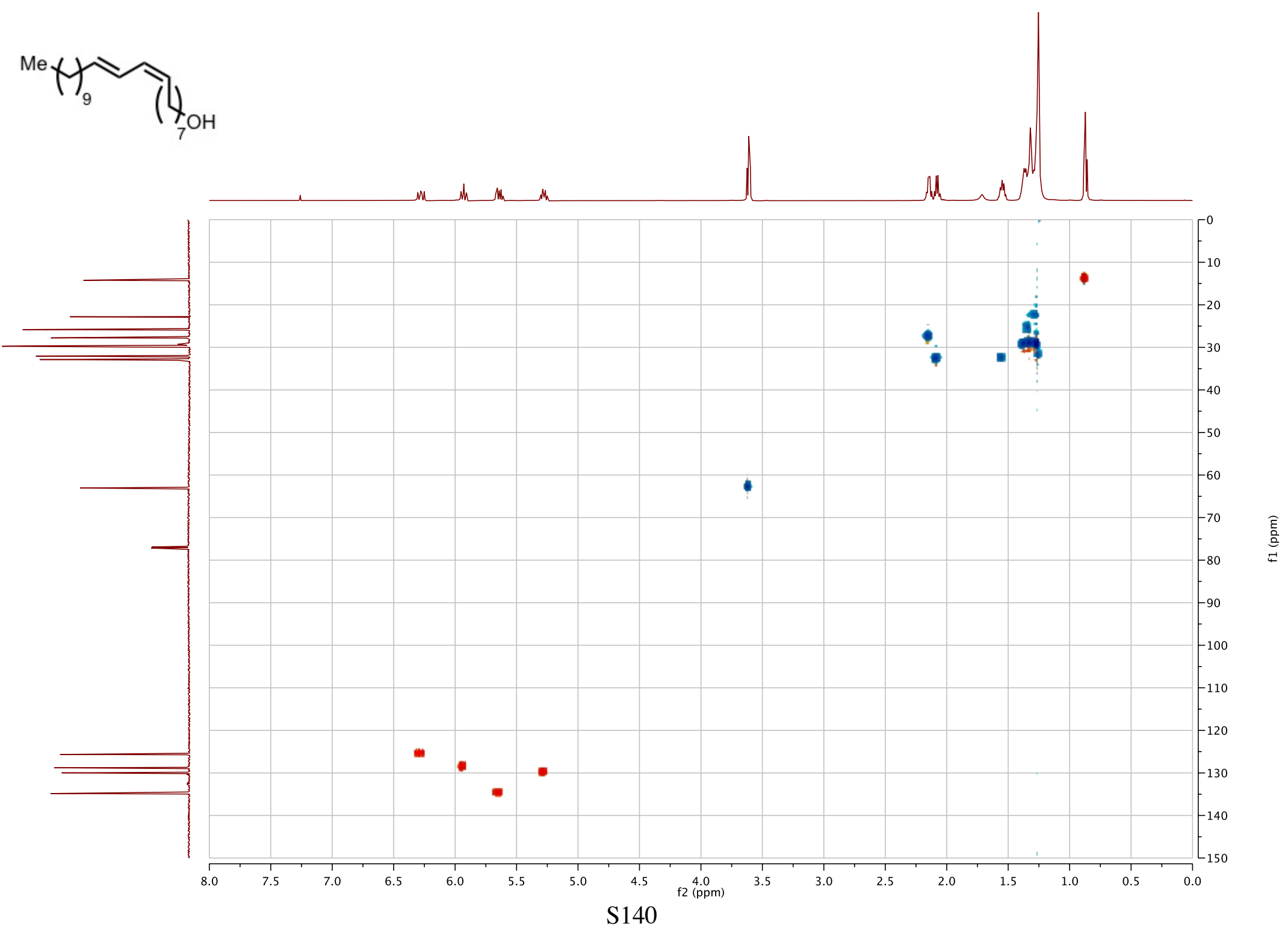


${ }^{1} \mathrm{H}$ NMR $\left(500 \mathrm{MHz}, \mathrm{CDCl}_{3}\right.$ ) spectrum of compound $9 \mathbf{b}$.

管<smiles>CC(C)(C)/C=C/C=C\COC/C=C/c1ccccc1</smiles>
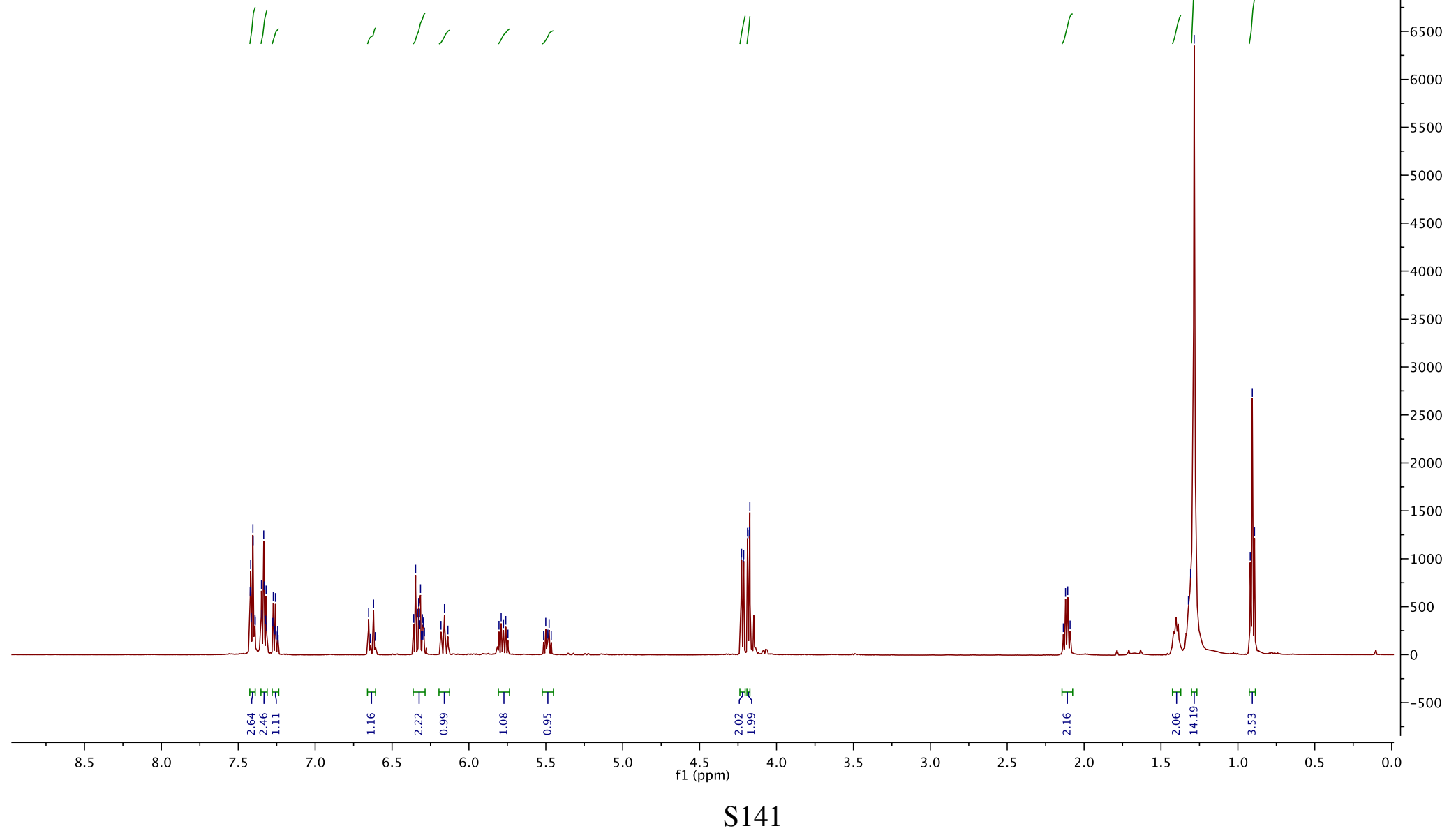
${ }^{13} \mathrm{C}$ NMR (126 MHz, $\mathrm{CDCl}_{3}$ ) spectrum of compound $\mathbf{9 b}$.

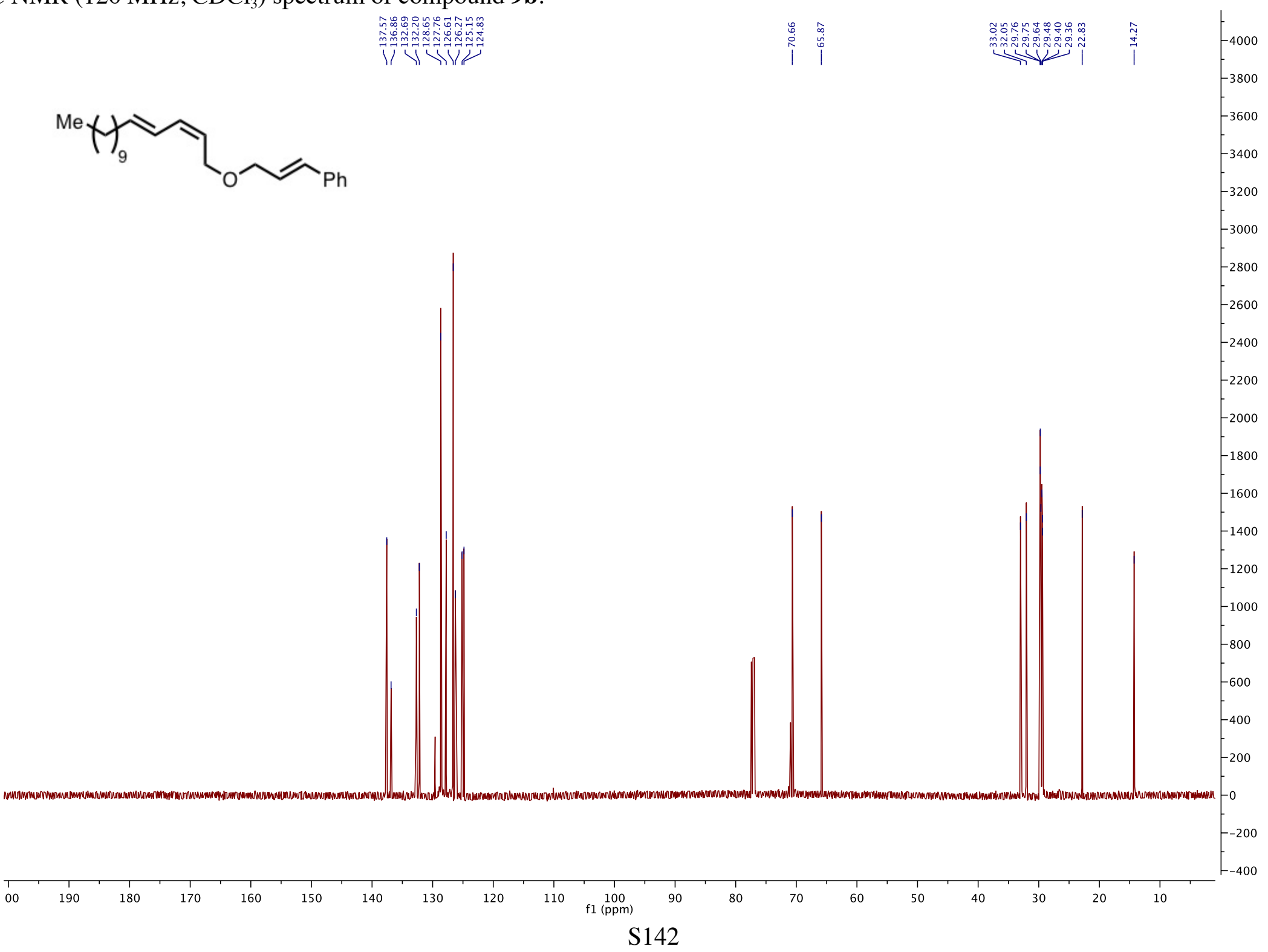


gCOSY spectrum of compound $\mathbf{9 b}$.

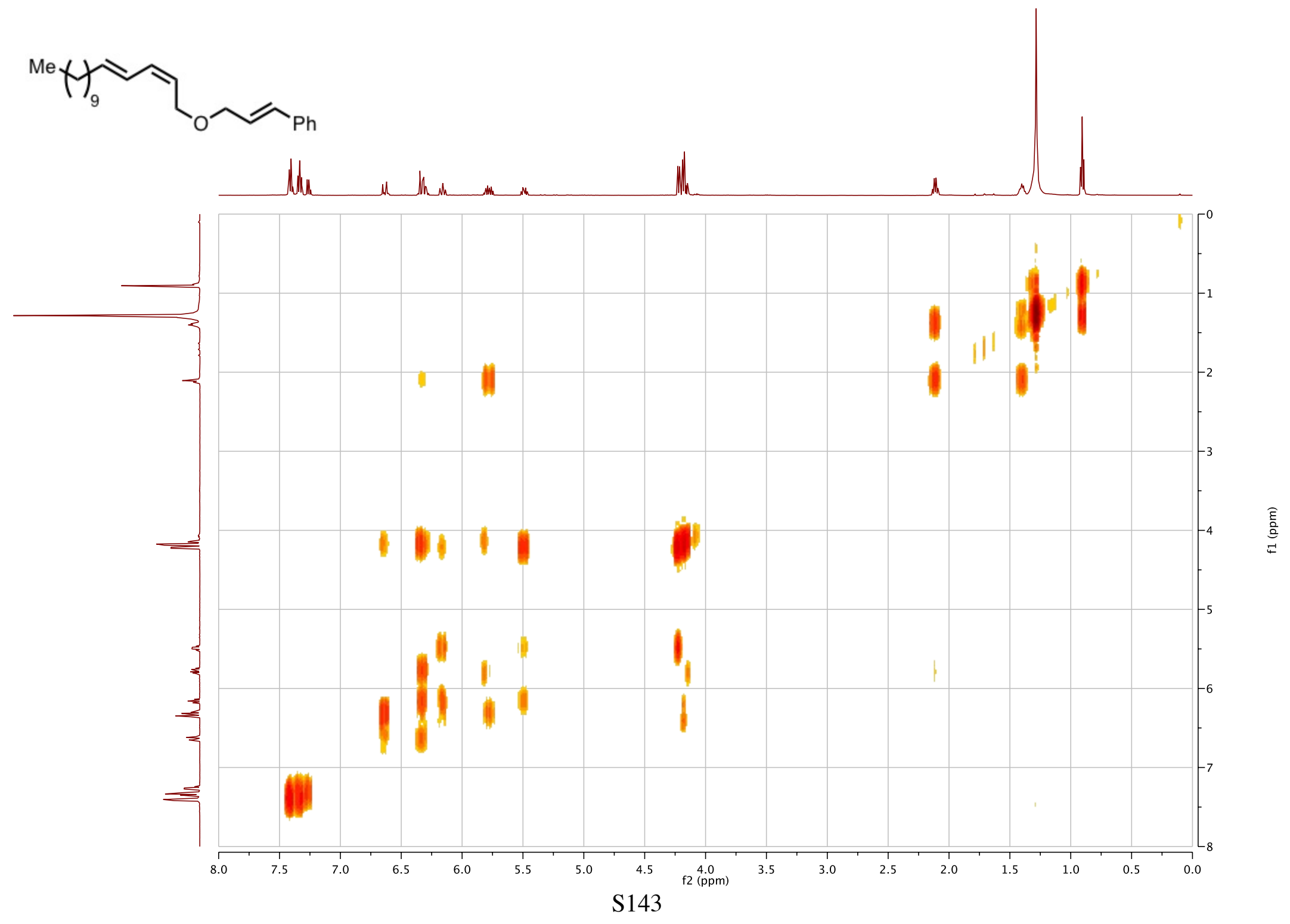


HSQC spectrum of compound $\mathbf{9 b}$.

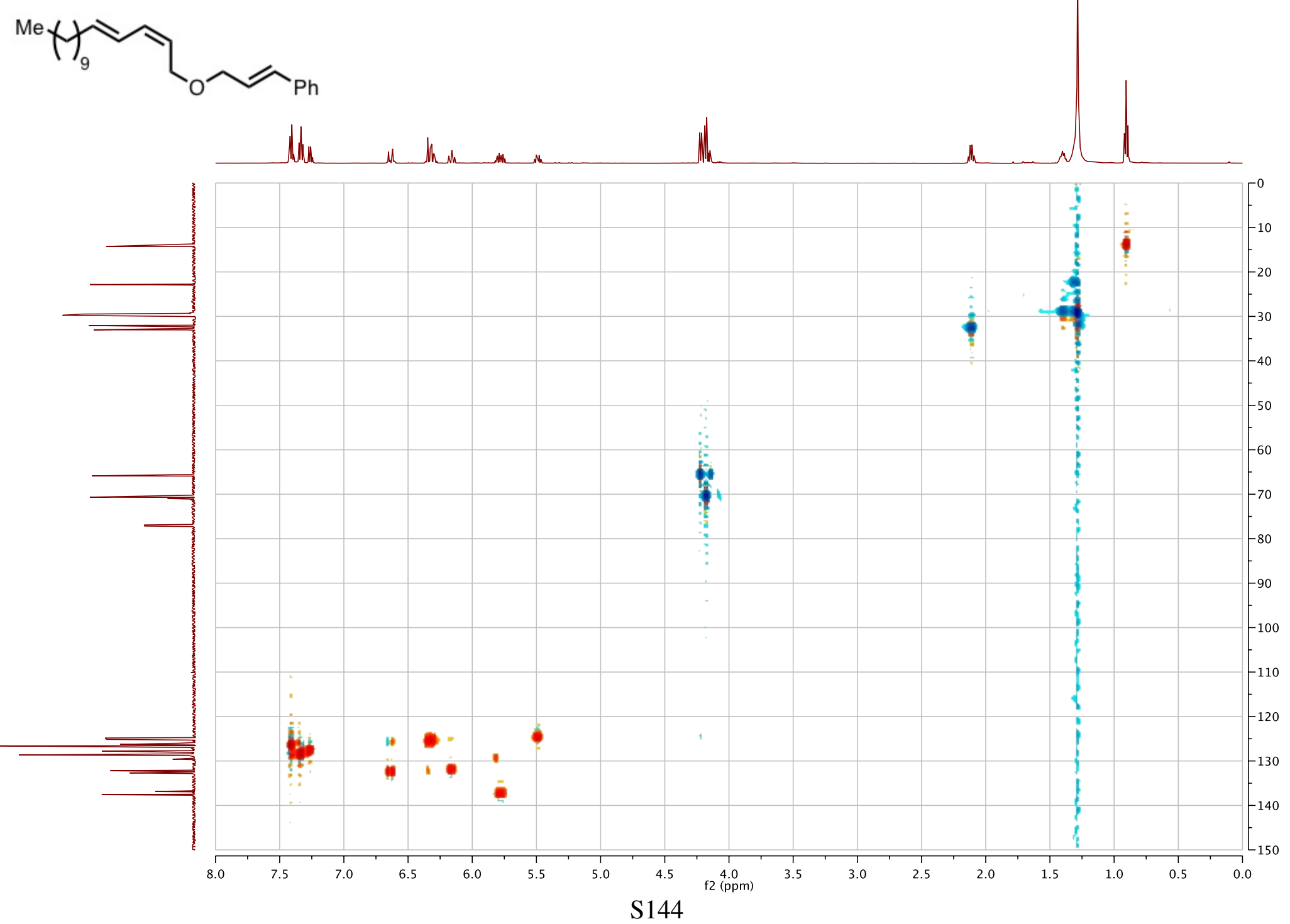


${ }^{1} \mathrm{H}$ NMR (400 MHz, $\mathrm{CDCl}_{3}$ ) spectrum of compound 9c.

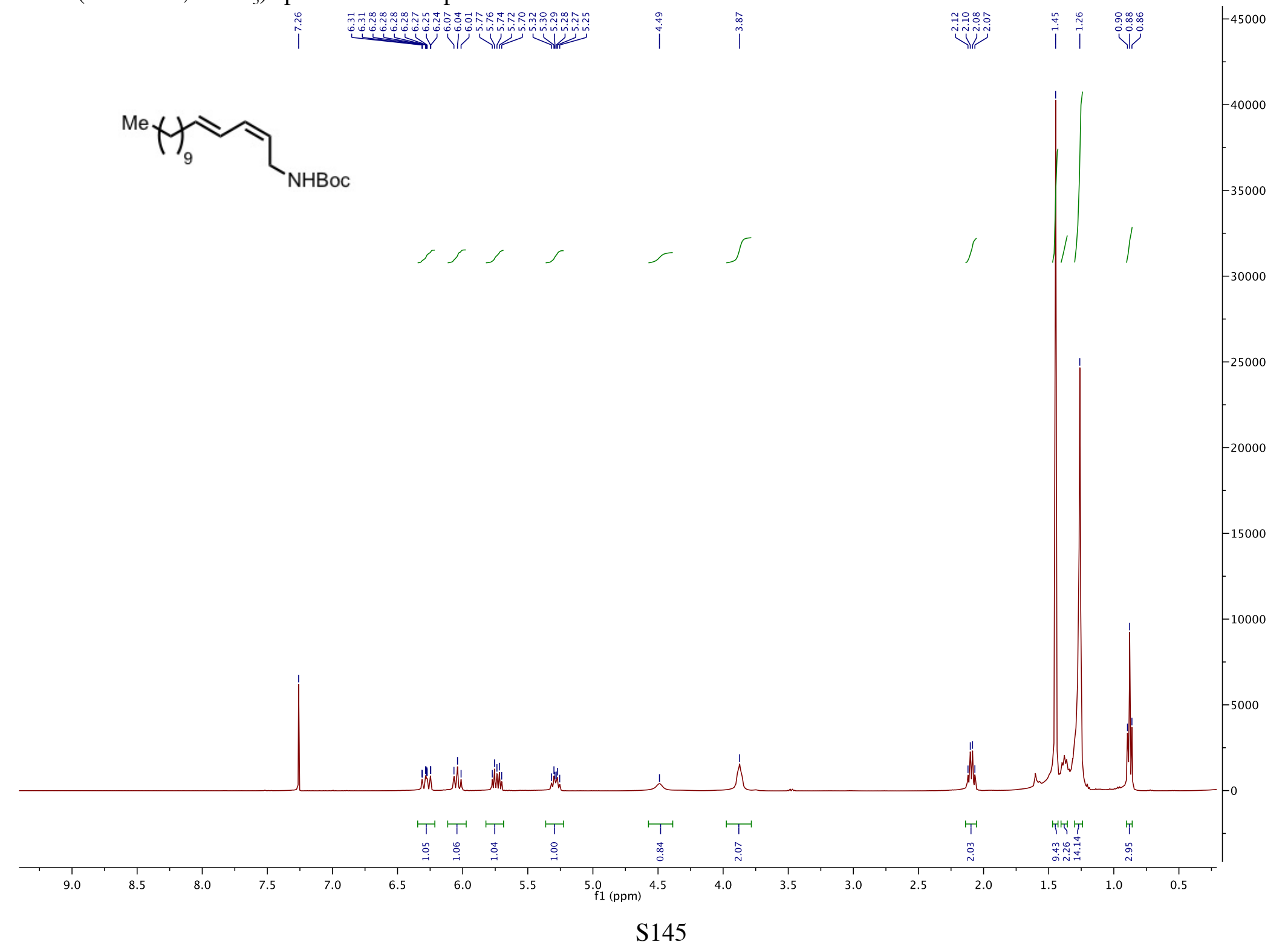


${ }^{13} \mathrm{C} \mathrm{NMR}\left(101 \mathrm{MHz}, \mathrm{CDCl}_{3}\right)$ spectrum of compound 9c.

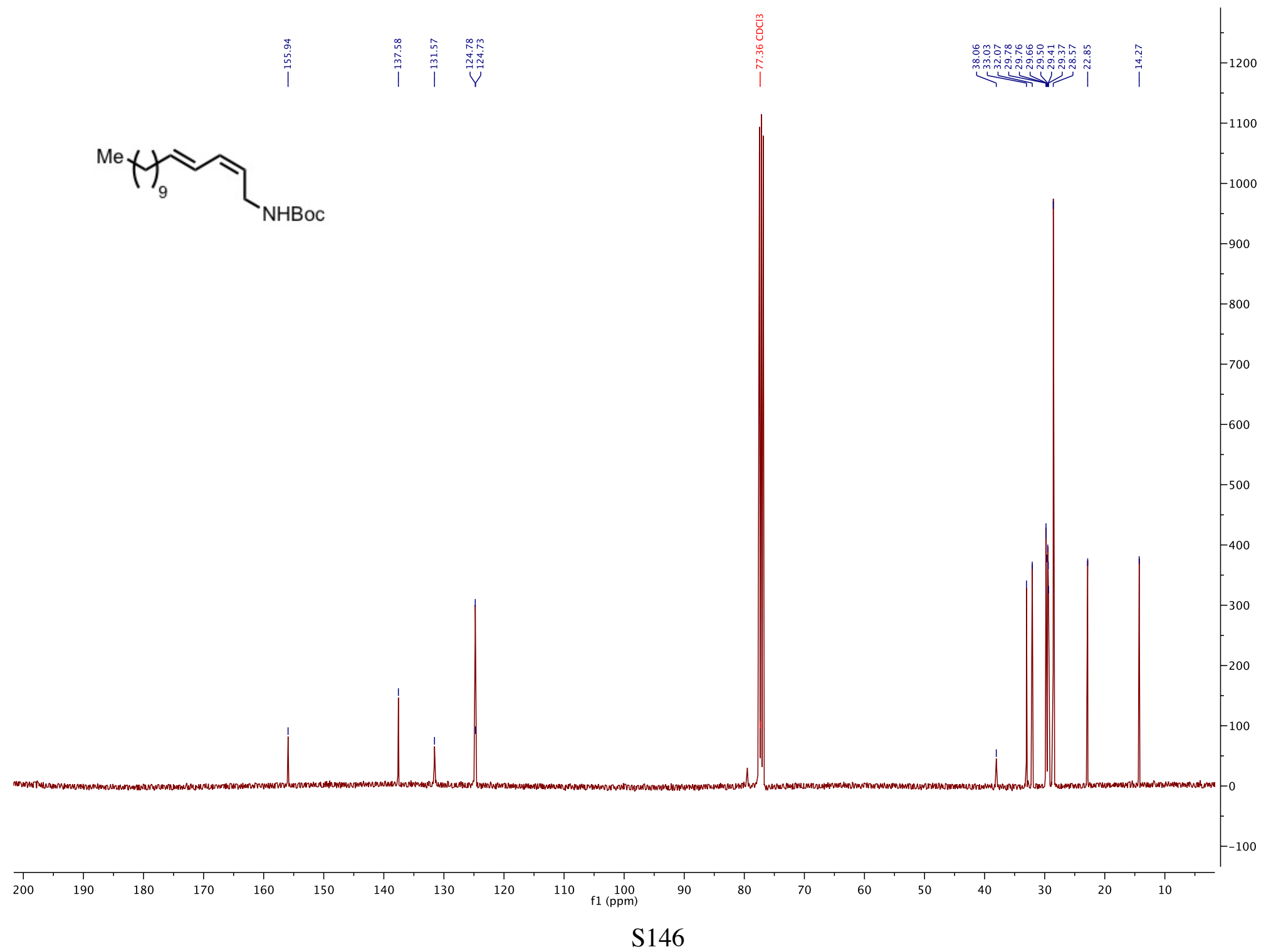


${ }^{1} \mathrm{H}$ NMR (400 MHz, $\mathrm{CDCl}_{3}$ ) spectrum of compound 9d.

ฟị̂

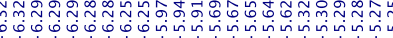

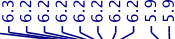<smiles>CC(C)C=CC=CCC(C)C=O</smiles>

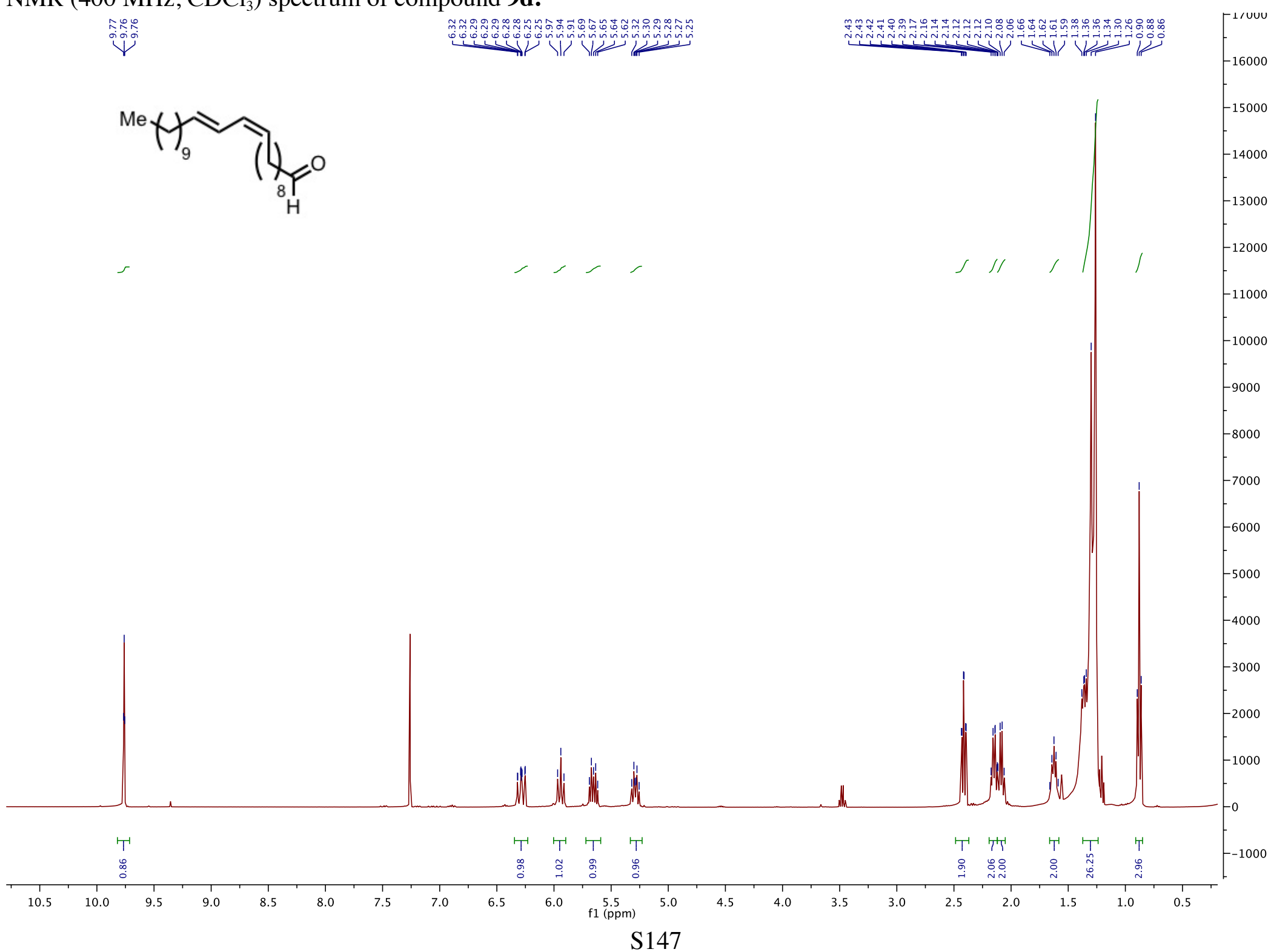


${ }^{13} \mathrm{C}$ NMR (101 MHz, $\mathrm{CDCl}_{3}$ ) spectrum of compound 9d.

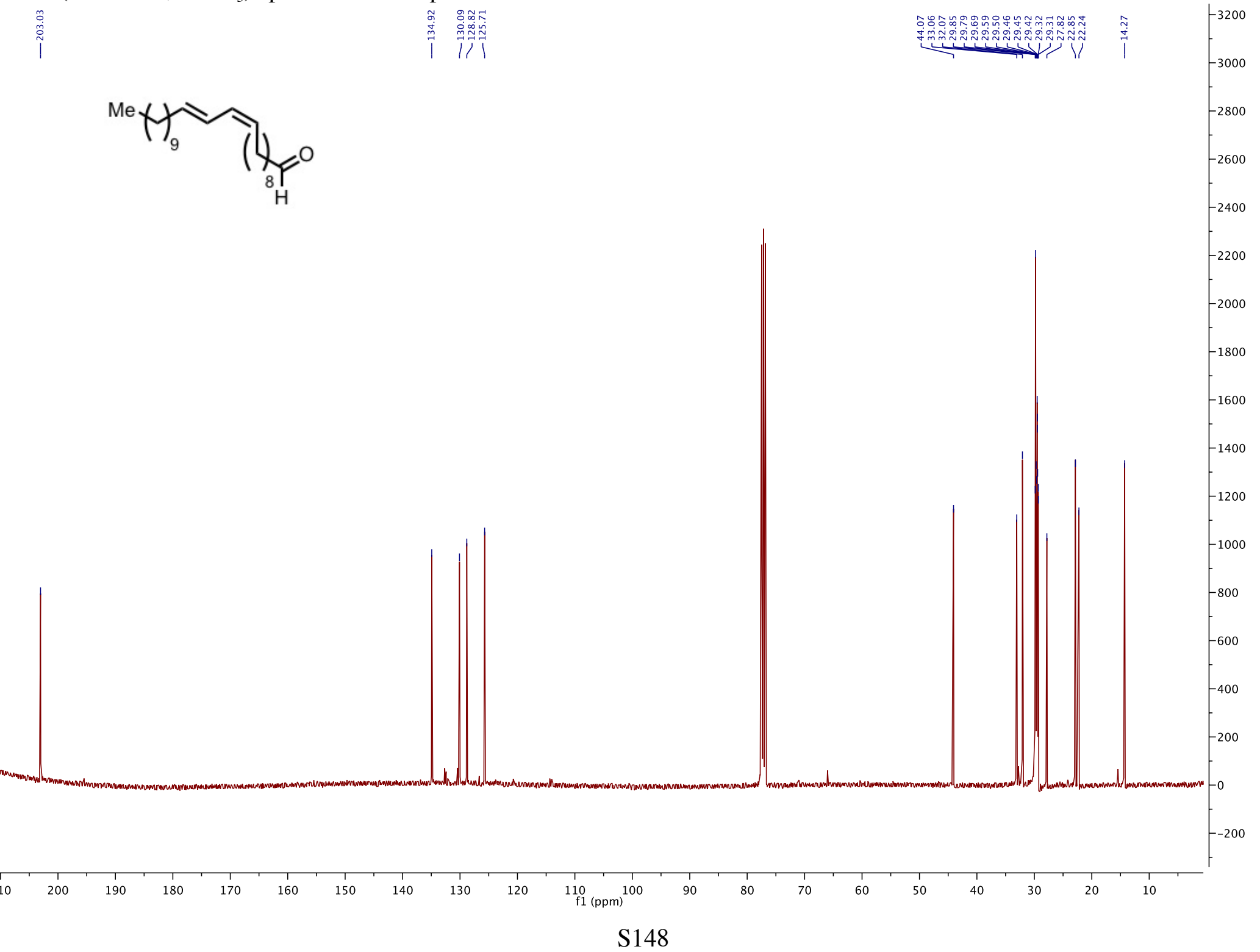


${ }^{1} \mathrm{H}$ NMR (400 MHz, $\mathrm{CDCl}_{3}$ ) spectrum of compound 9e. ( 94\% purity)

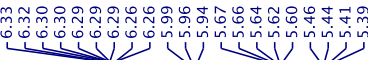
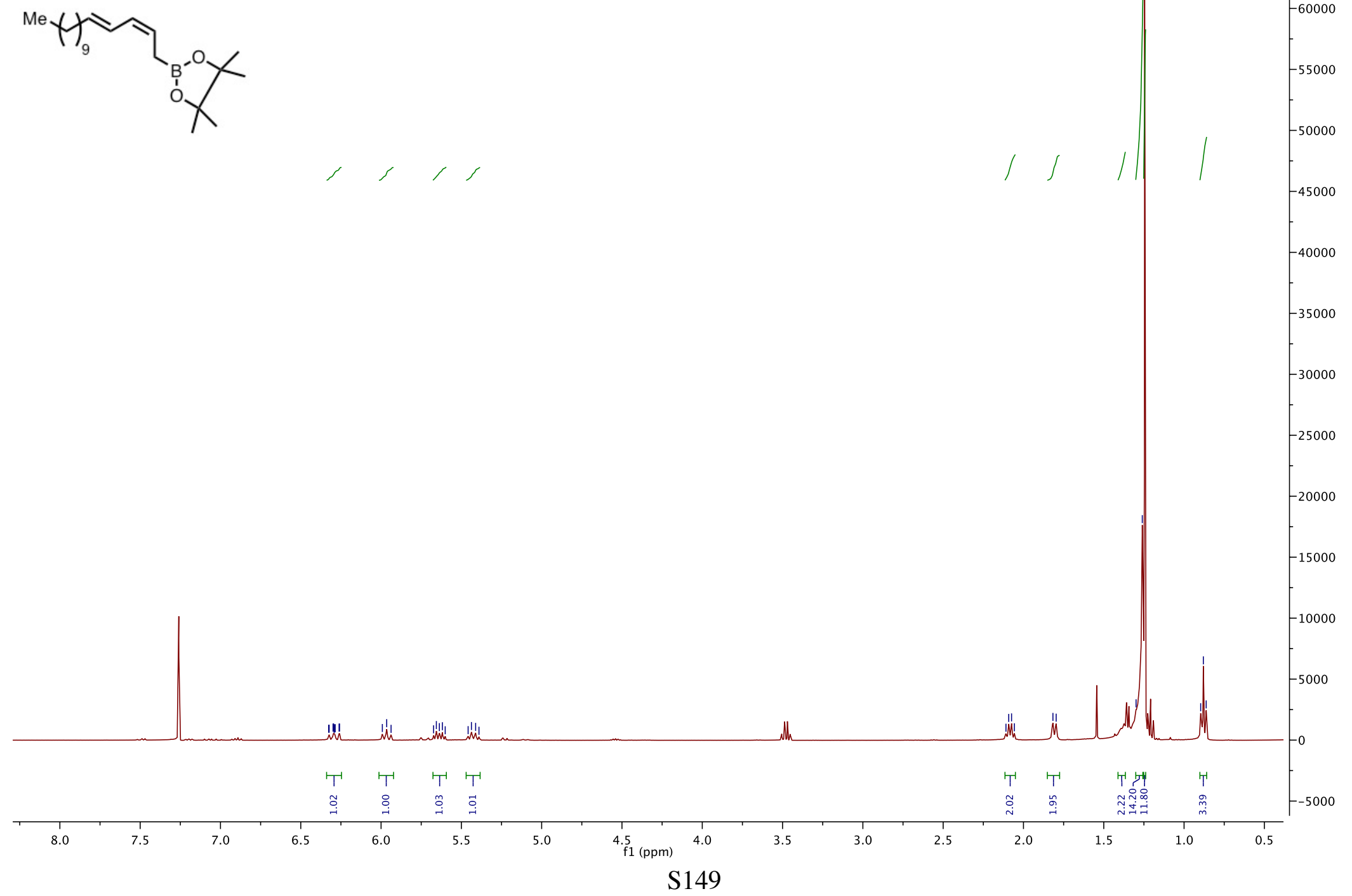
${ }^{1} \mathrm{H}$ NMR (400 MHz, $\mathrm{CDCl}_{3}$ ) spectrum of crude reaction mixture of compound 9e with nitrobenzene internal standard.

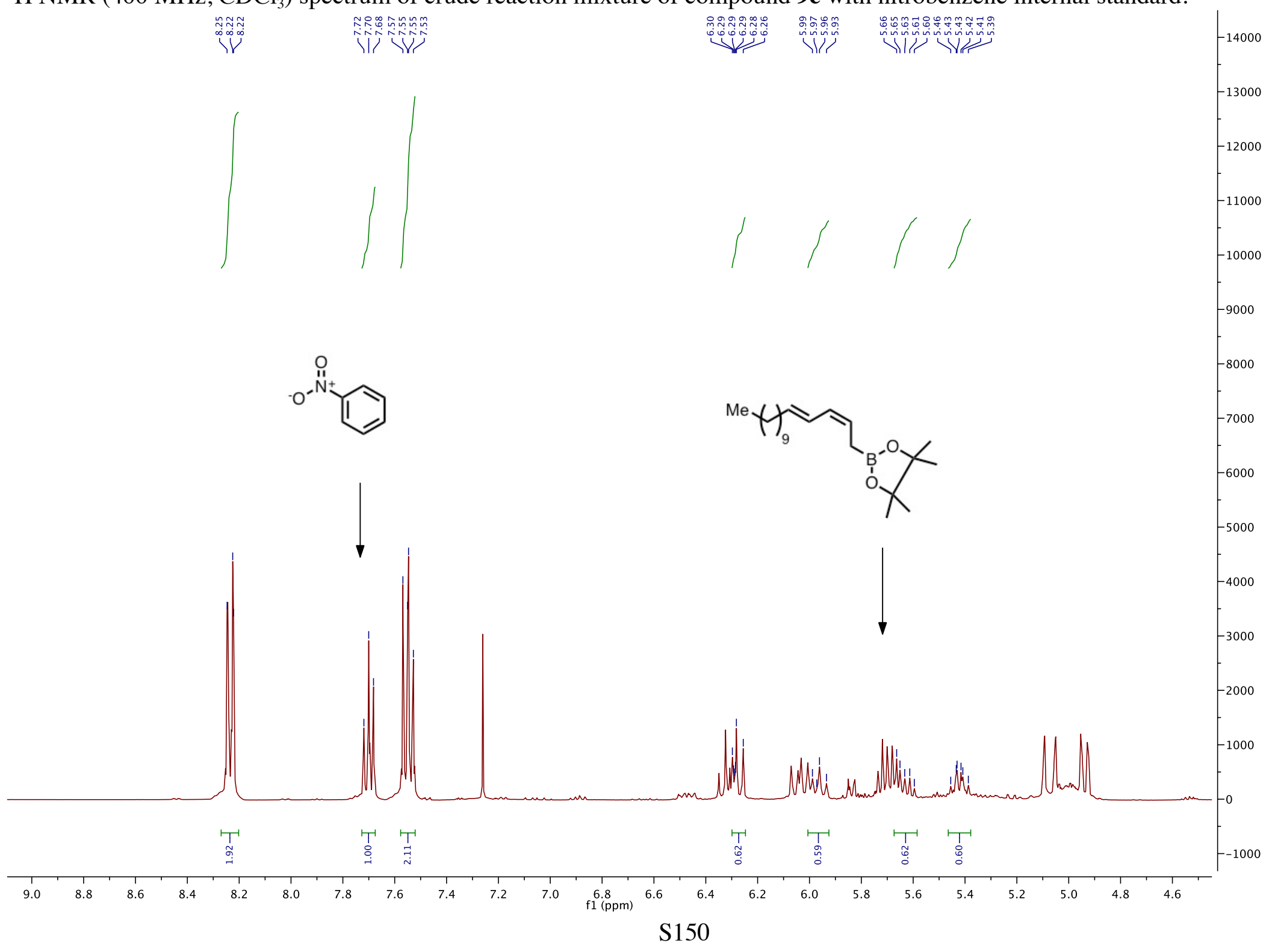


${ }^{1} \mathrm{H} \mathrm{NMR}\left(400 \mathrm{MHz}, \mathrm{CDCl}_{3}\right.$ ) spectrum of compound 9 f.

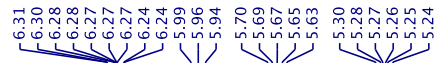<smiles>COC(=O)C=CC=CC=CC(C)(C)C</smiles>

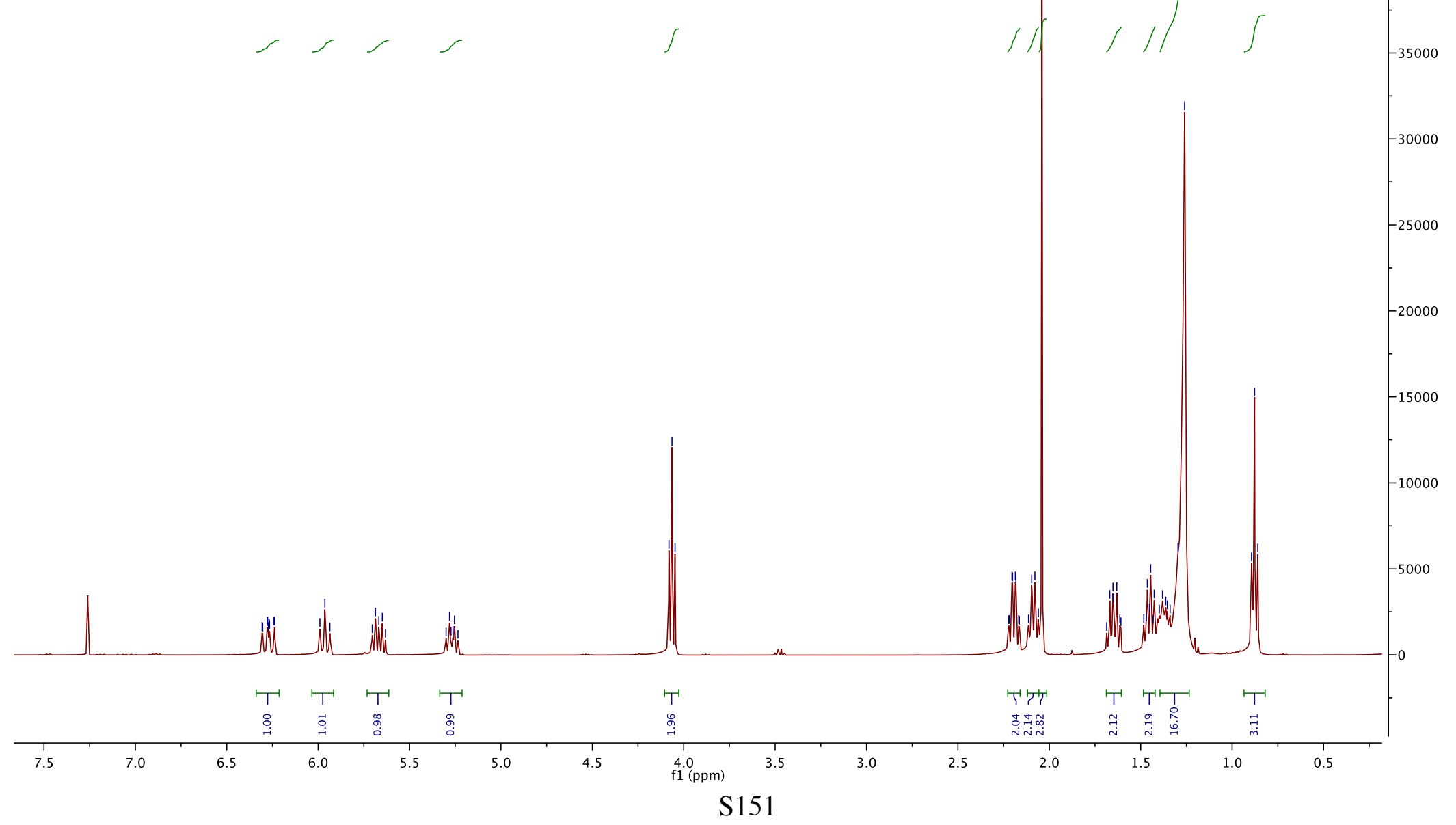

$-50000$ 
${ }^{13} \mathrm{C}$ NMR (101 MHz, $\mathrm{CDCl}_{3}$ ) spectrum of compound 9 f.

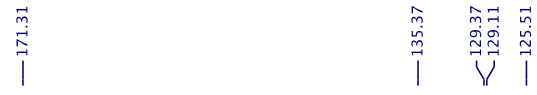

$\mathrm{Me}+\mathrm{l}_{4}$

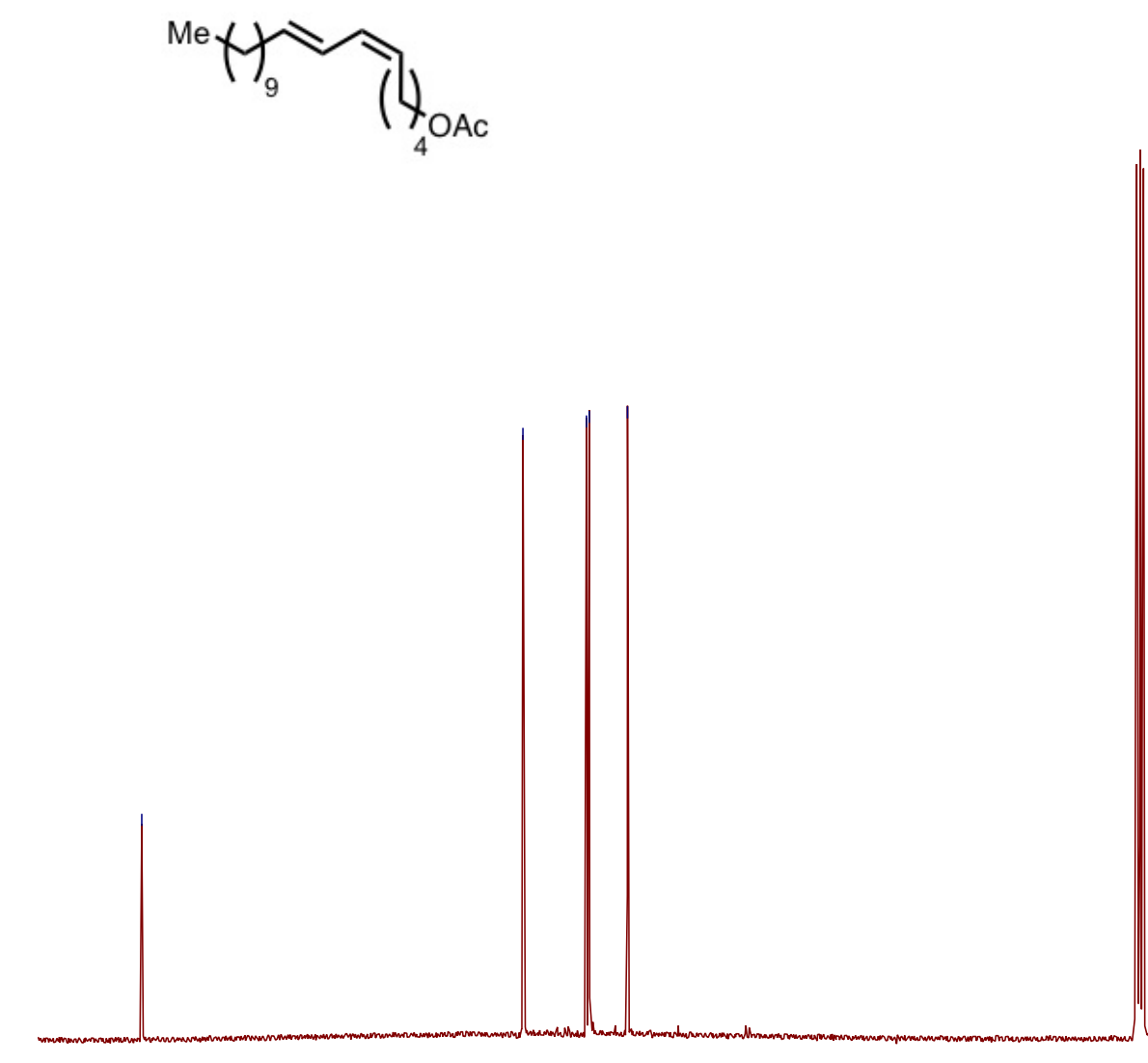

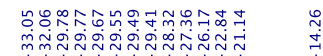

$-5000$

4500

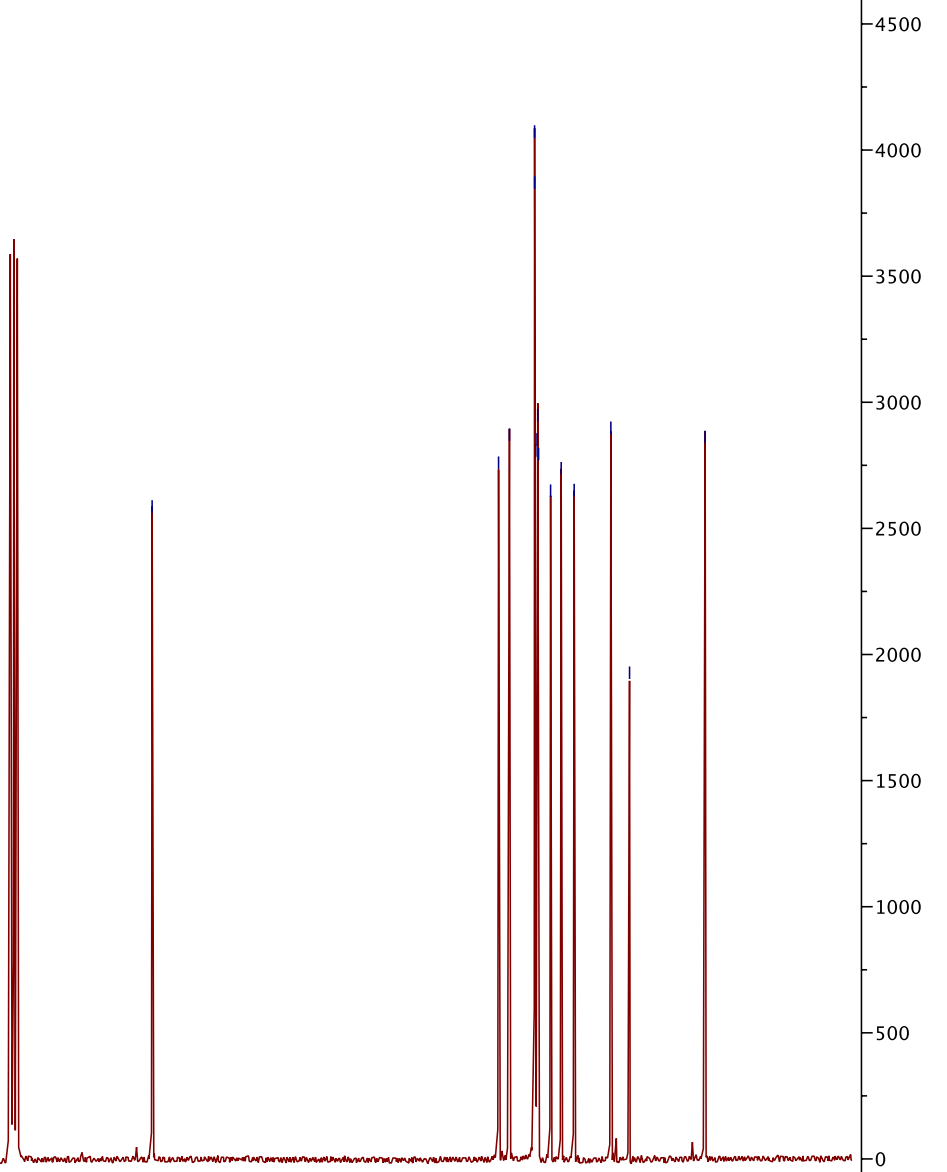

$\underset{180}{\top}$

170

160

150

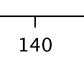

130

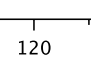

110

100 
${ }^{1} \mathrm{H}$ NMR (400 MHz, $\mathrm{CDCl}_{3}$ ) spectrum of compound 9g.

舟
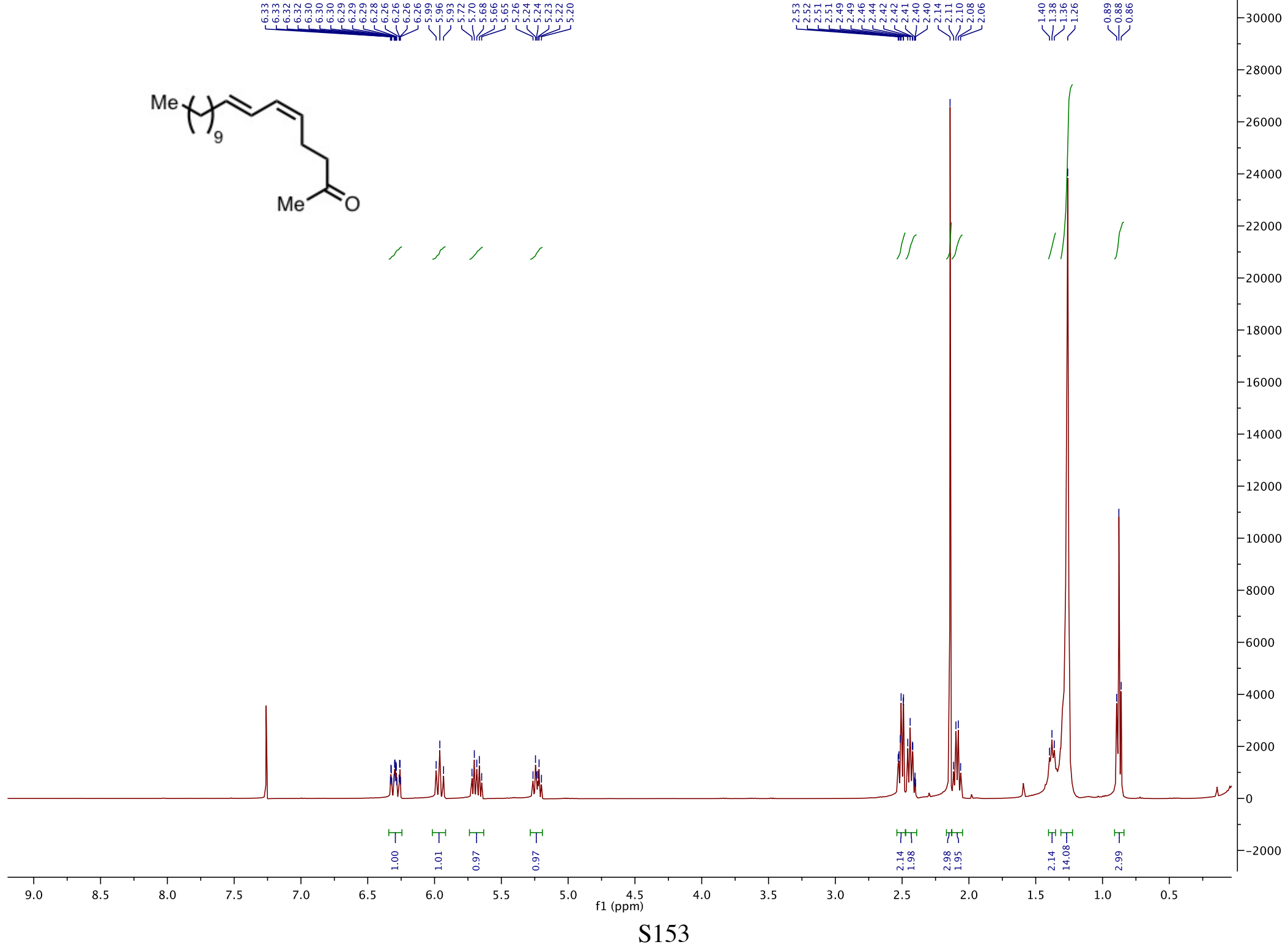
${ }^{13} \mathrm{C}$ NMR (101 MHz, $\mathrm{CDCl}_{3}$ ) spectrum of compound 9g.

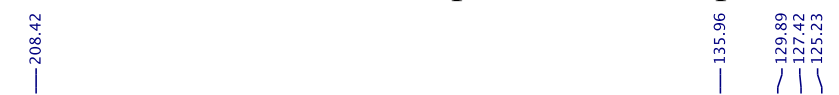

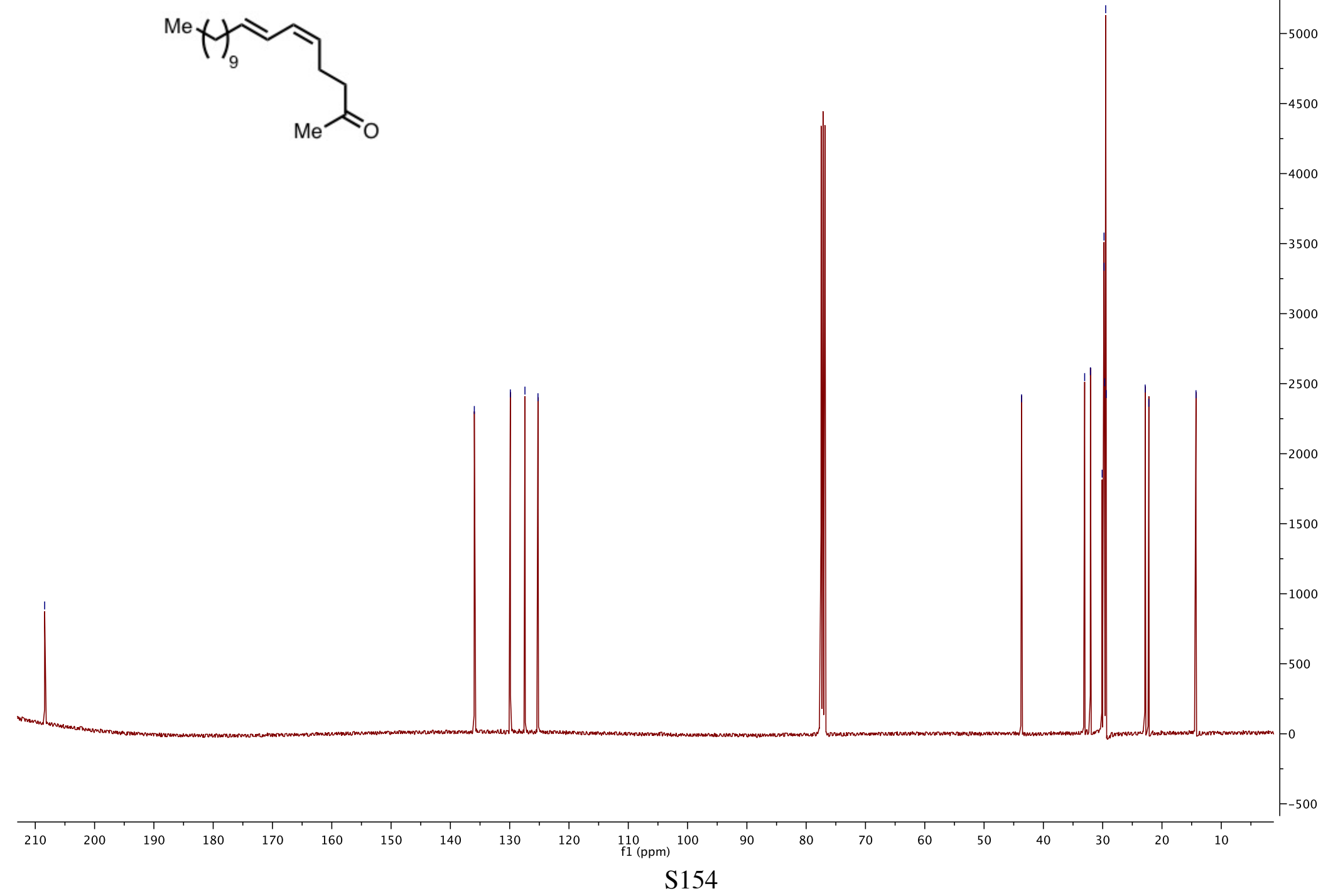


${ }^{1} \mathrm{H}$ NMR (400 MHz, $\mathrm{CDCl}_{3}$ ) spectrum of compound $\mathbf{9 h}$.

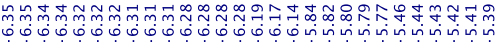<smiles>COC(=O)OC/C=C\C=C\C(C)(C)C</smiles>

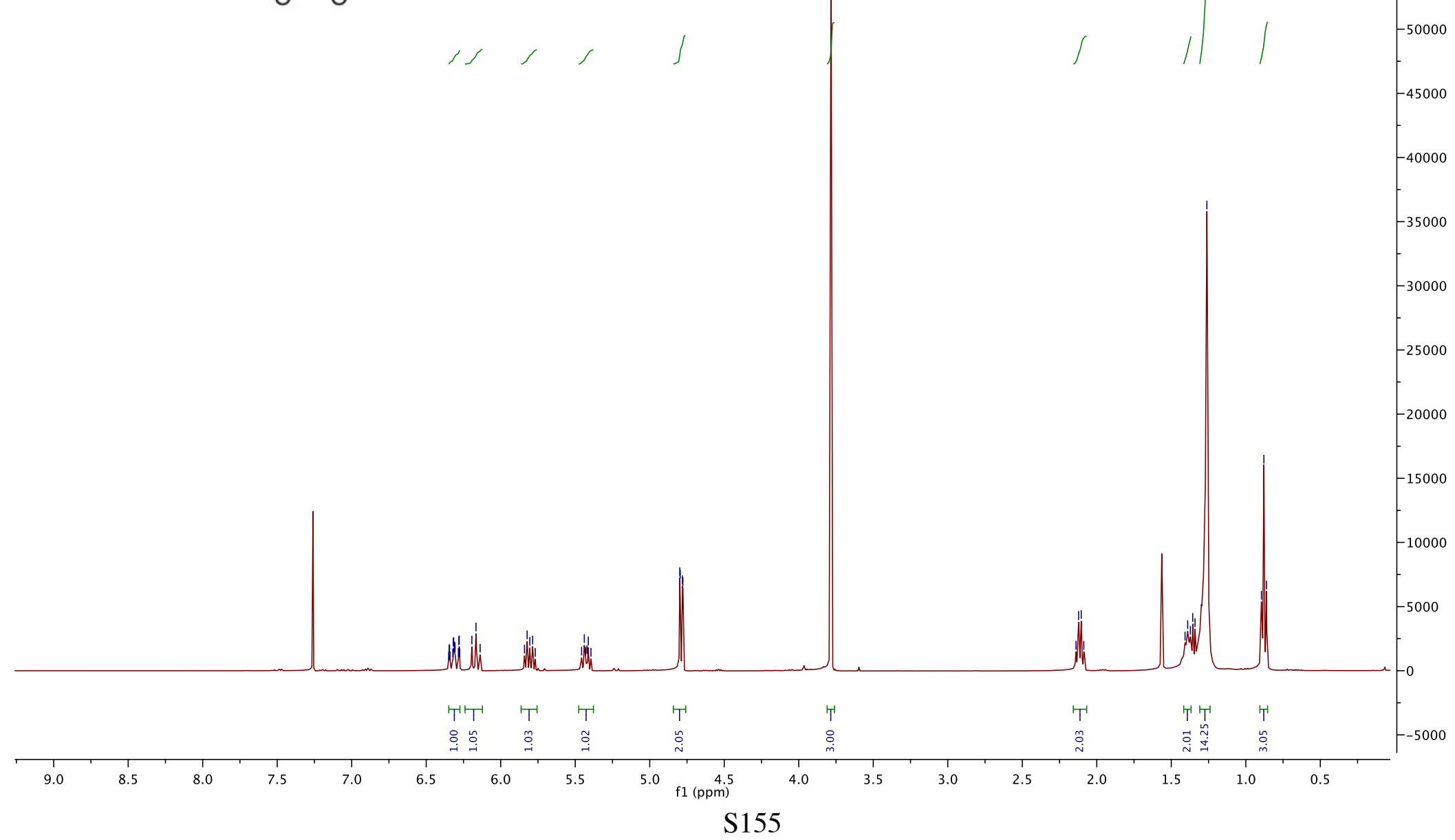


${ }^{13} \mathrm{C}$ NMR (101 MHz, $\mathrm{CDCl}_{3}$ ) spectrum of compound $\mathbf{9 h}$.

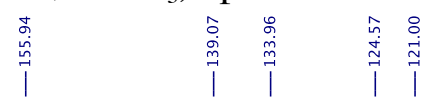<smiles>COC(=O)OC/C=C\C=C\C(C)C(C)C</smiles>

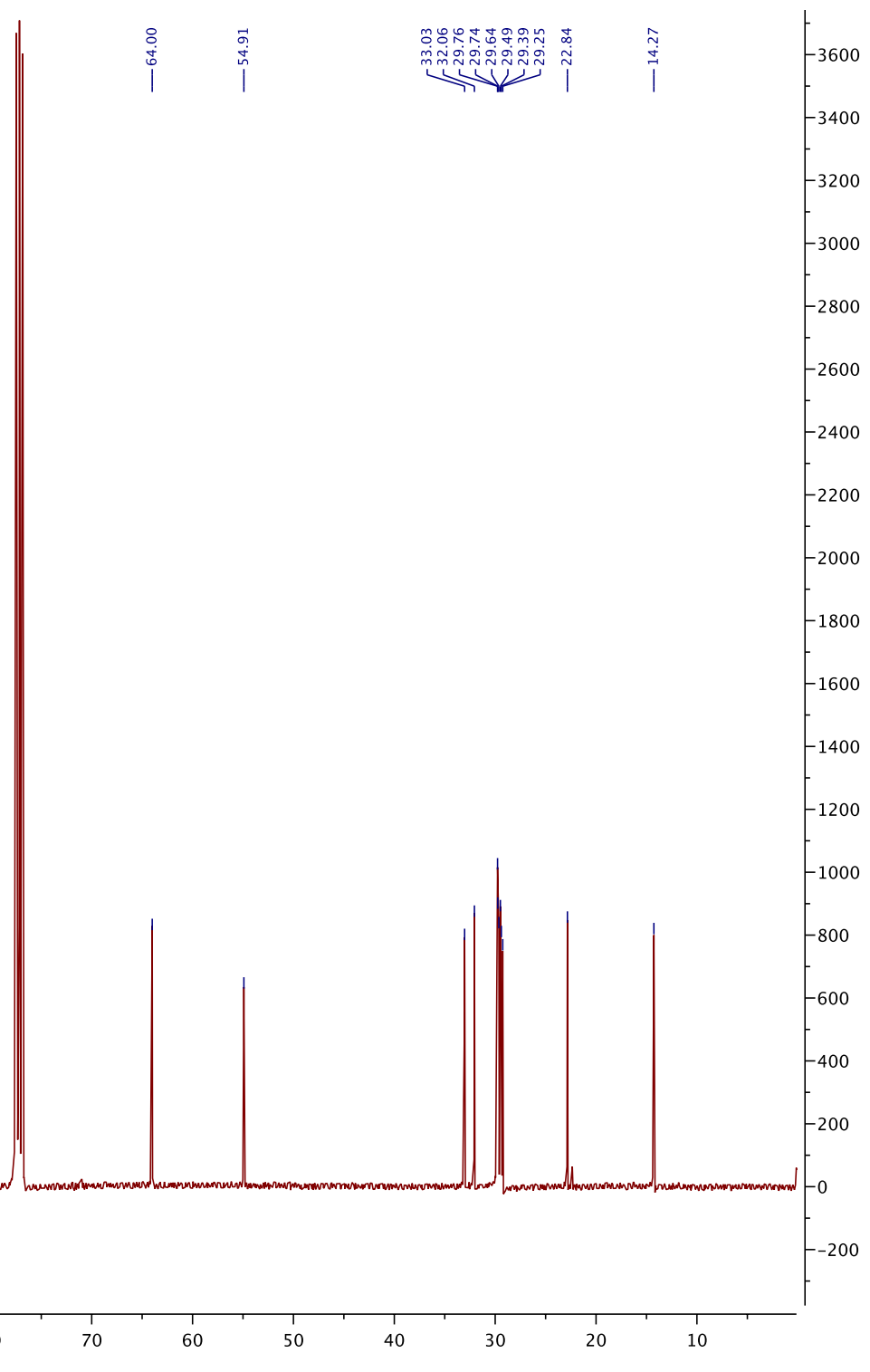


${ }^{1} \mathrm{H}$ NMR (400 MHz, $\mathrm{CDCl}_{3}$ ) spectrum of compound 9i.

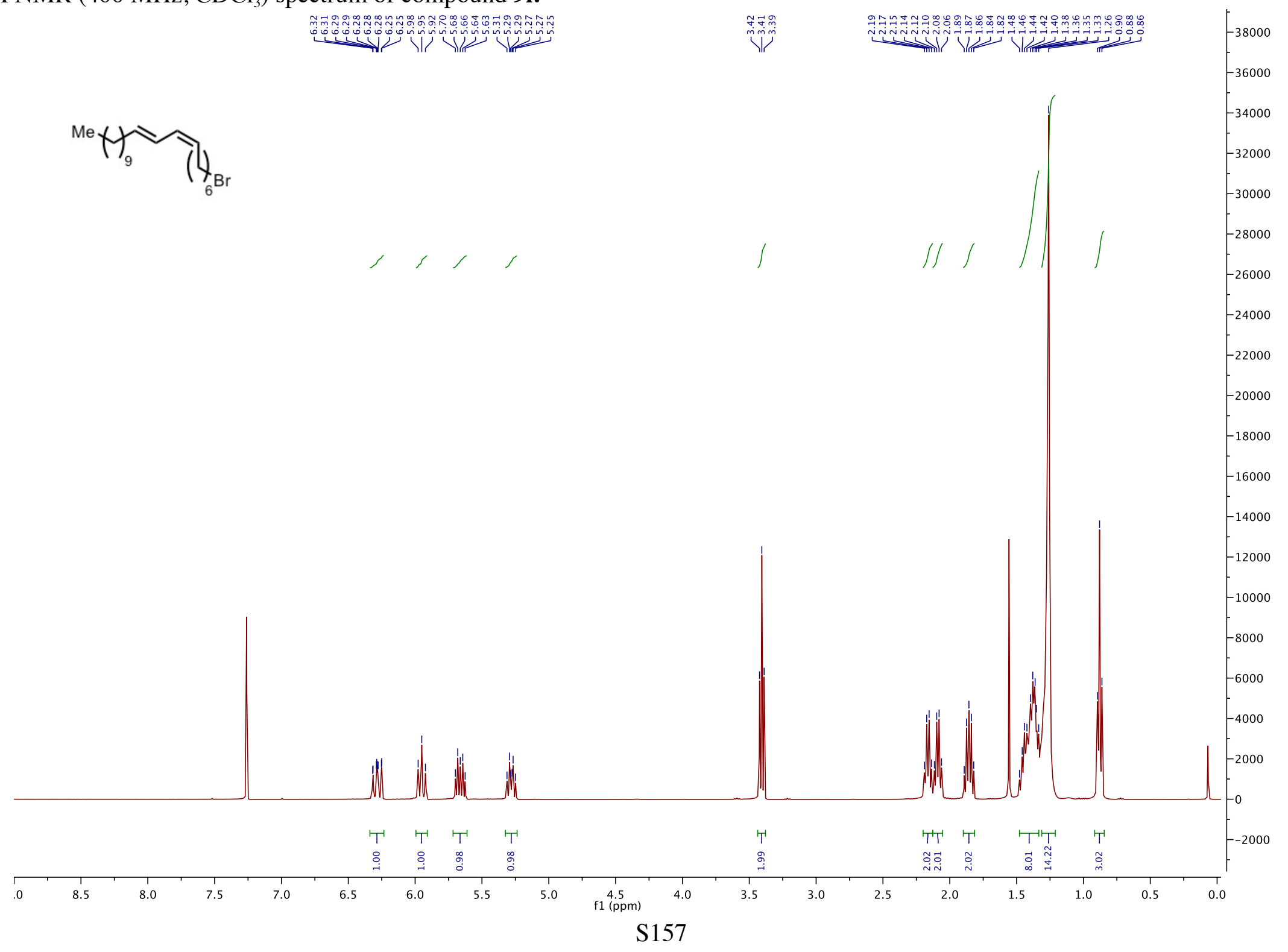


${ }^{13} \mathrm{C}$ NMR (101 MHz, $\mathrm{CDCl}_{3}$ ) spectrum of compound $\mathbf{9 i}$.

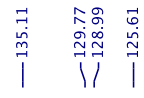

$\mathrm{Me}+\mathrm{H}_{6 \mathrm{Br}}$
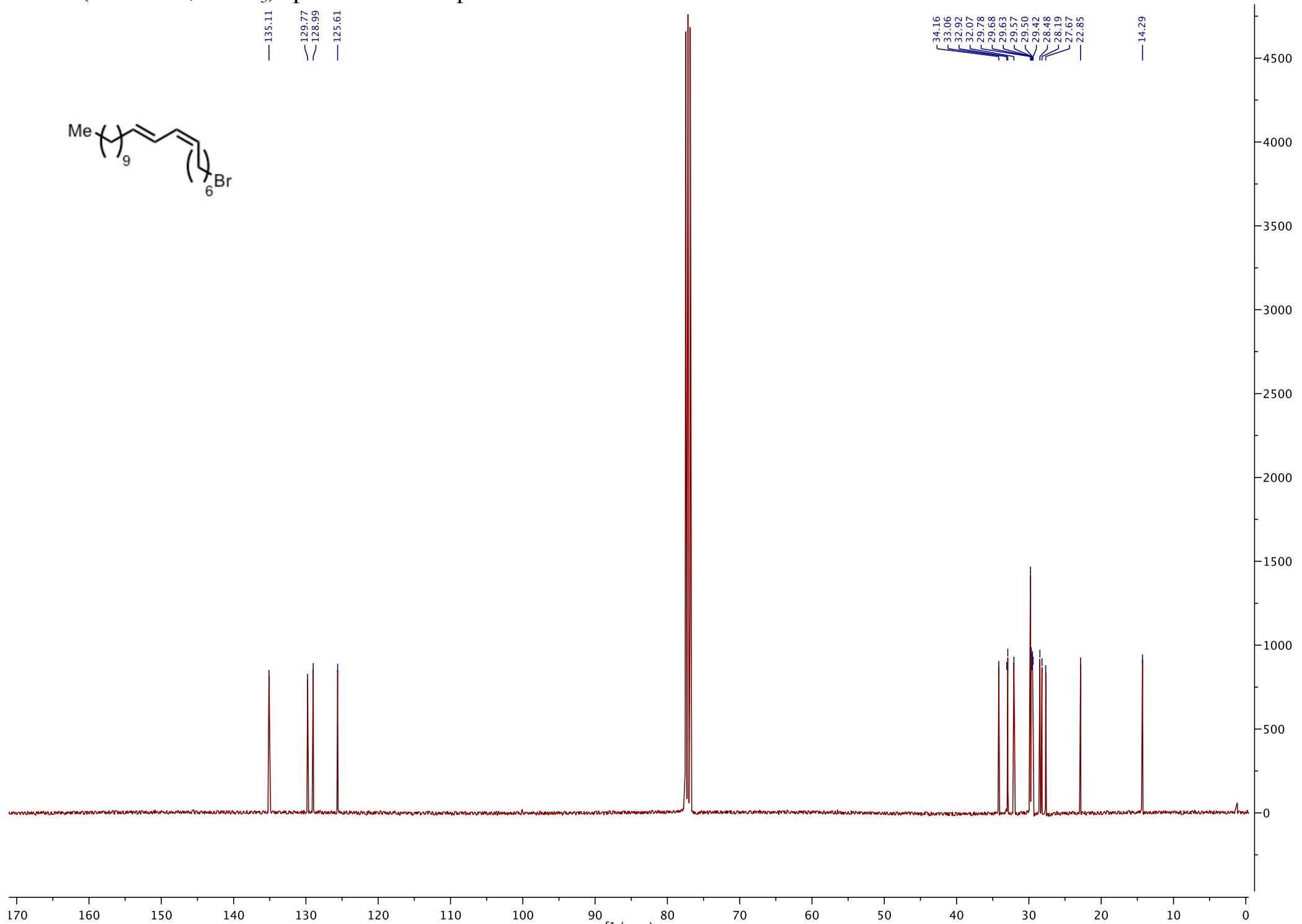

160

150

140

$130 \quad 120$

110

100

${ }_{90}^{10} 80$

S158 
${ }^{1} \mathrm{H}$ NMR (400 MHz, $\mathrm{CDCl}_{3}$ ) spectrum of compound $\mathbf{9 j}$.

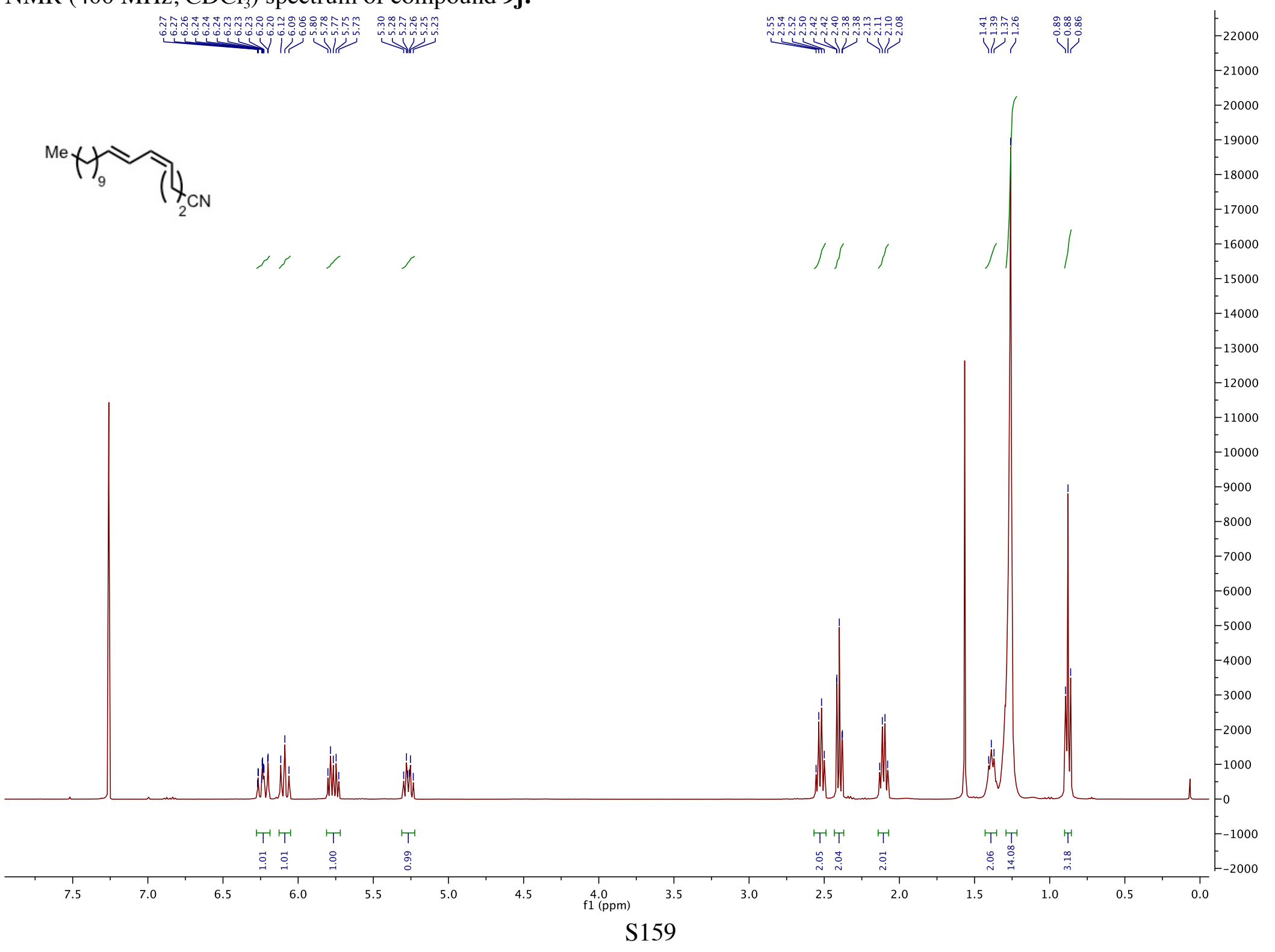


${ }^{13} \mathrm{C}$ NMR (101 MHz, $\mathrm{CDCl}_{3}$ ) spectrum of compound 9j.

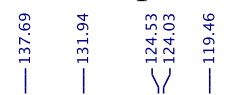<smiles>CC(C)C=CC=CCC#N</smiles>
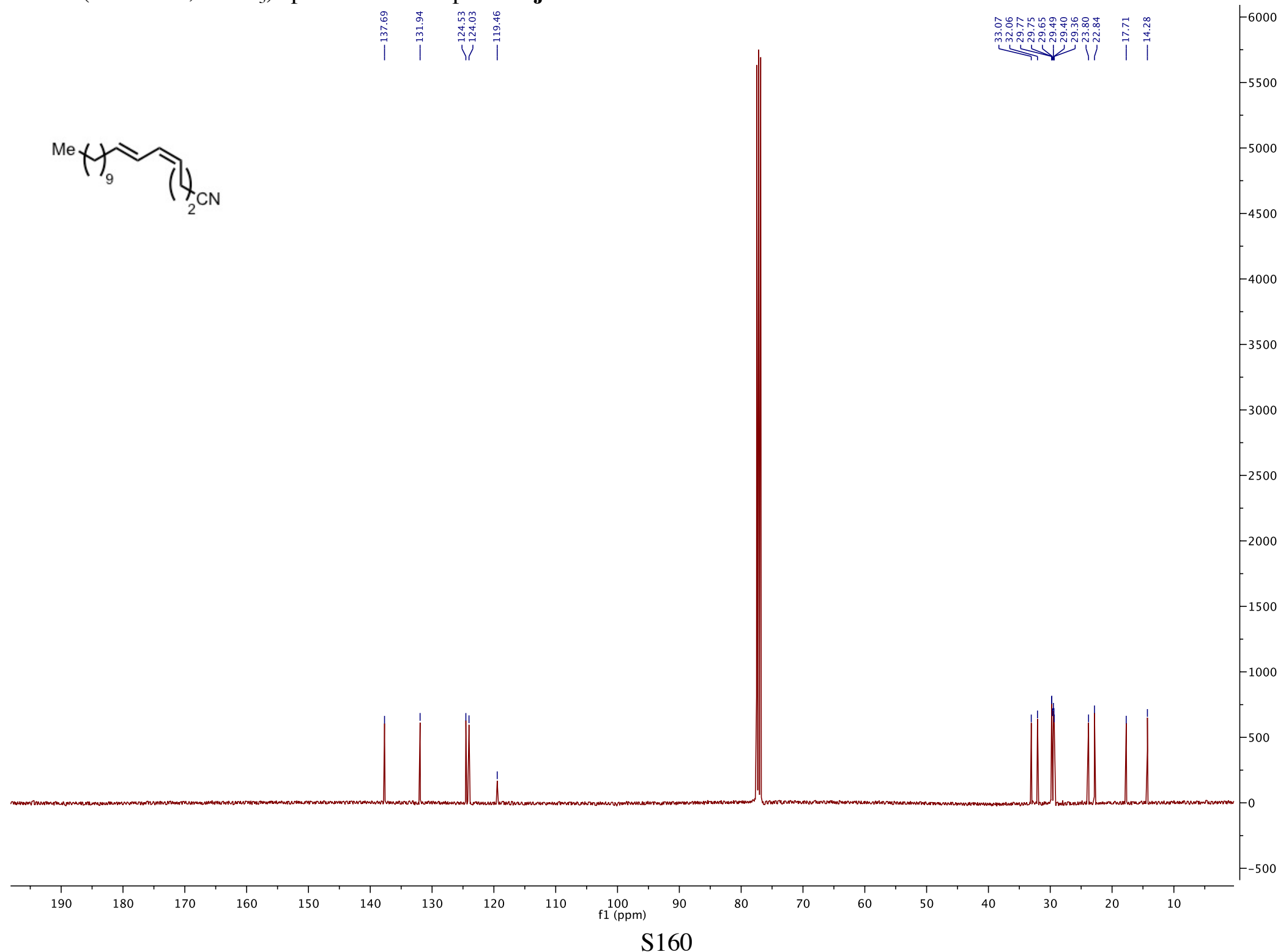
${ }^{1} \mathrm{H}$ NMR (500 MHz, $\mathrm{CDCl}_{3}$ ) spectrum of compound 9k.

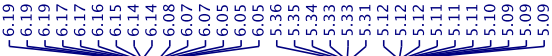<smiles>CC(C)=CCC/C(C)=C/C=C\C(=O)O</smiles>
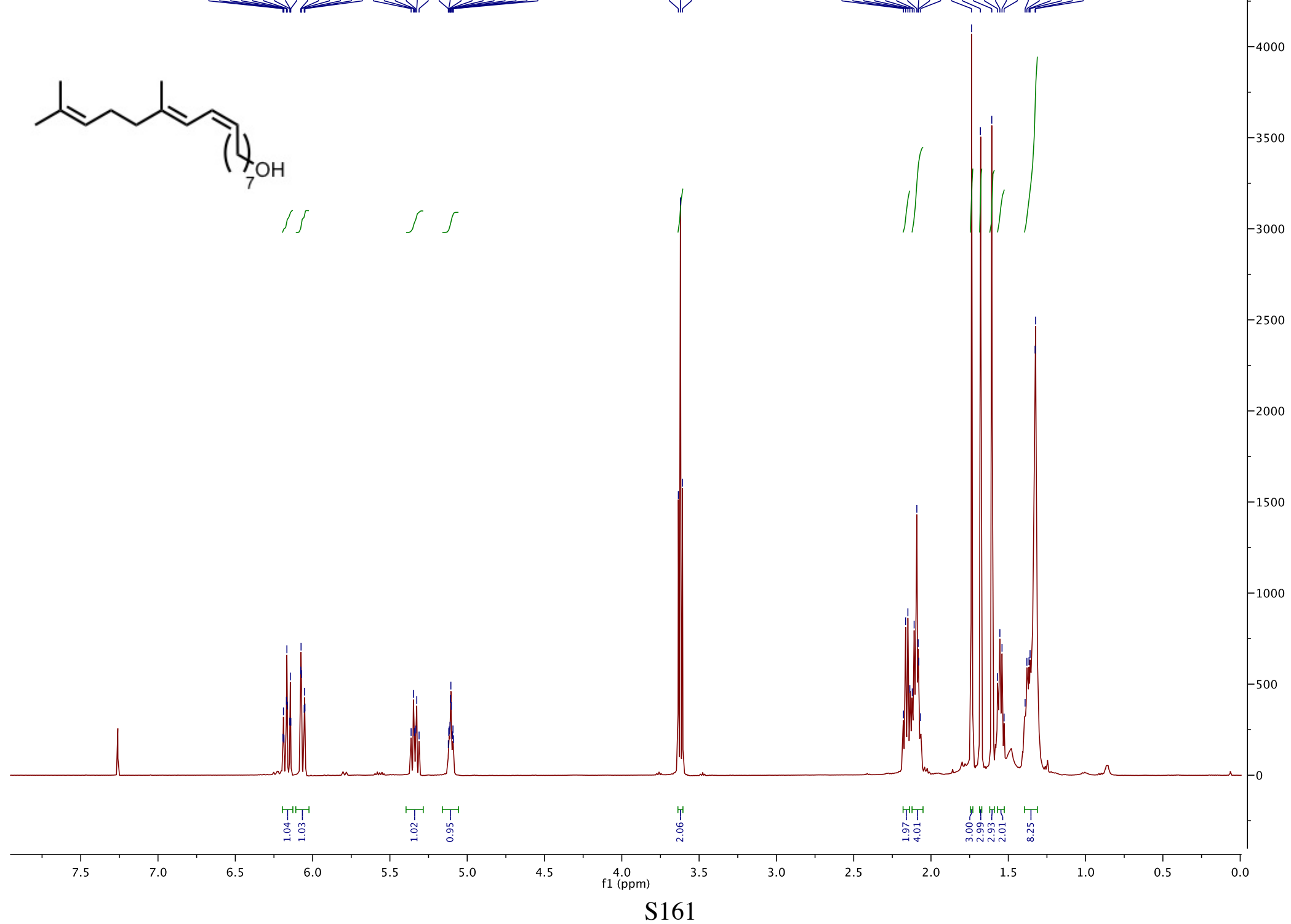
${ }^{13} \mathrm{C}$ NMR (126 MHz, $\mathrm{CDCl}_{3}$ ) spectrum of compound 9k.

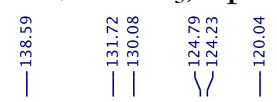
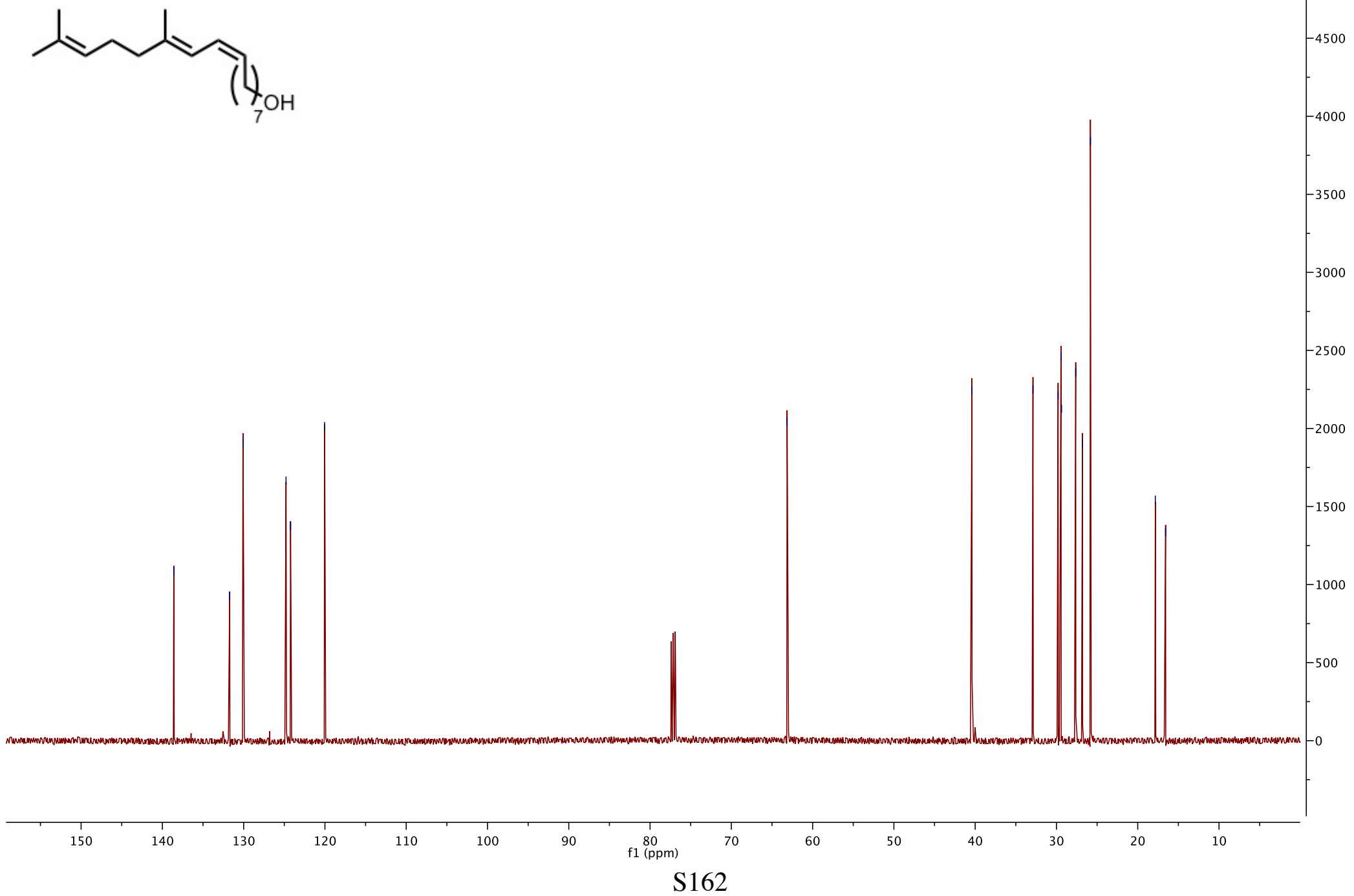
gCOSY spectrum of compound 9k.

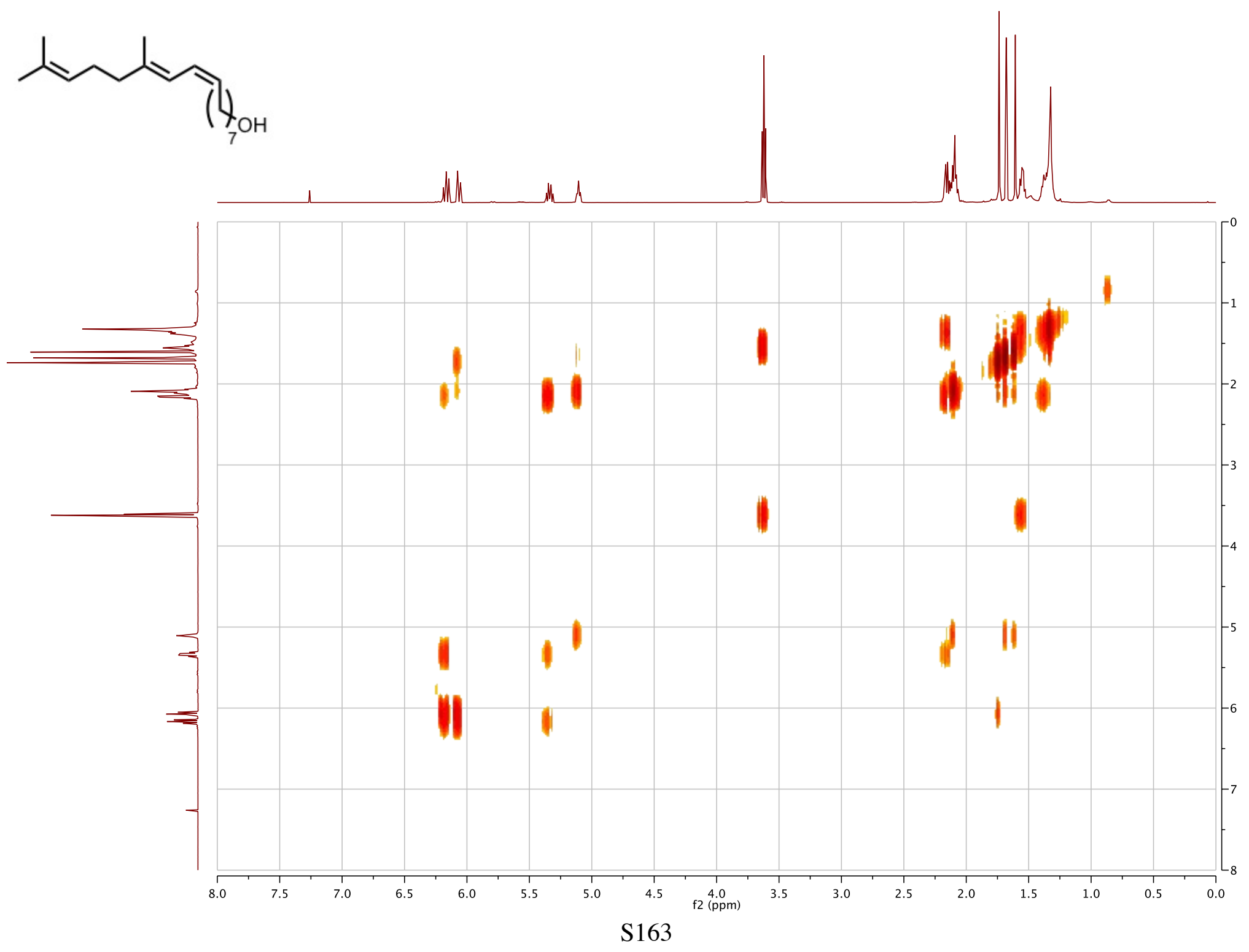


HSQC spectrum of compound 9k.

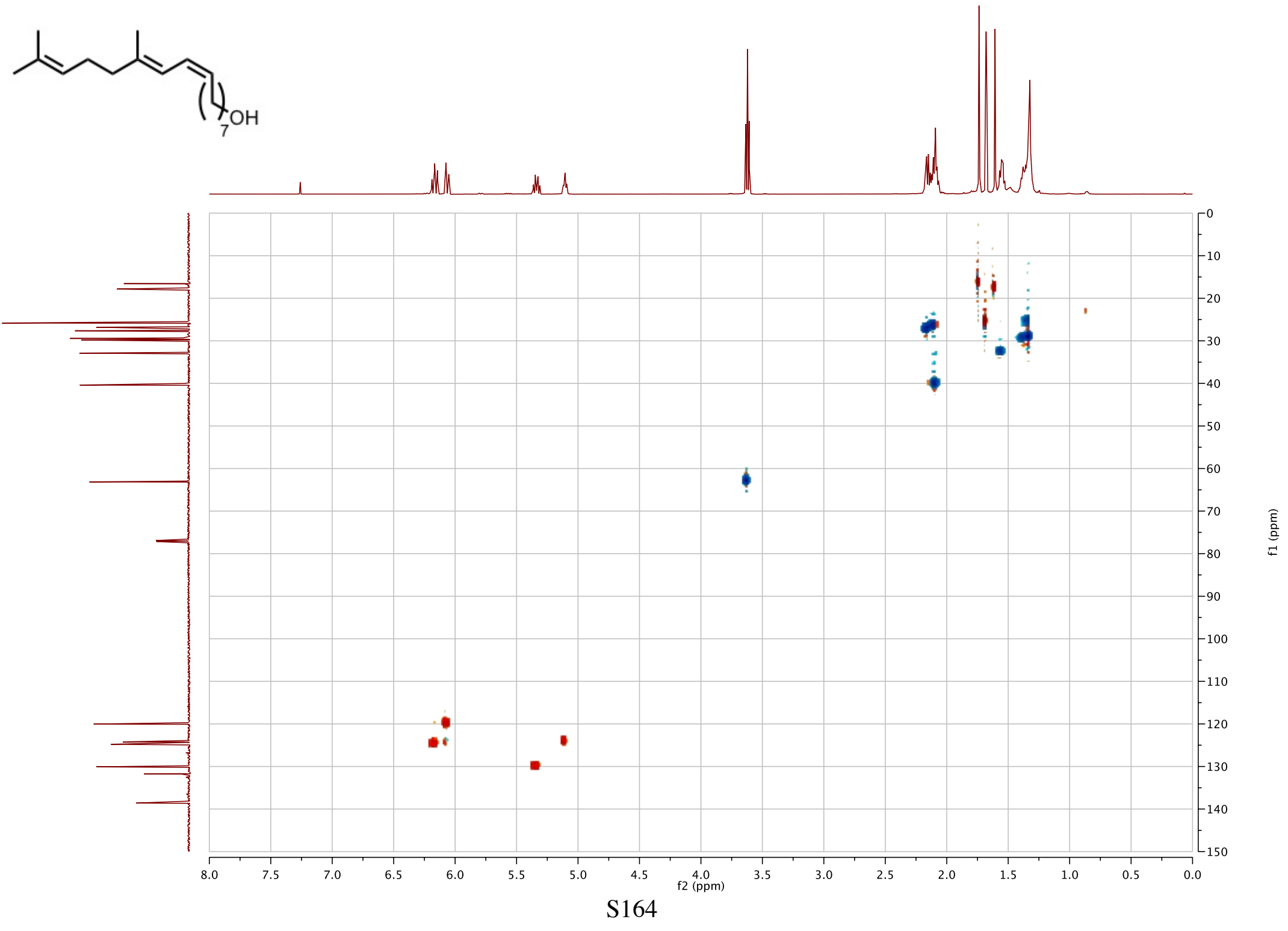


${ }^{1} \mathrm{H}$ NMR (400 MHz, $\mathrm{CDCl}_{3}$ ) spectrum of compound 91.

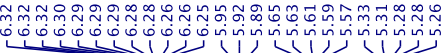

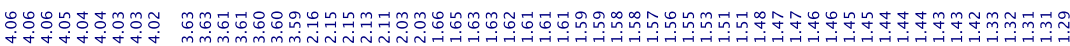

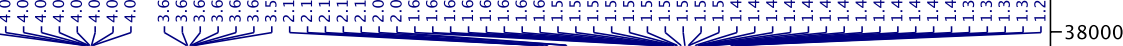

(4)

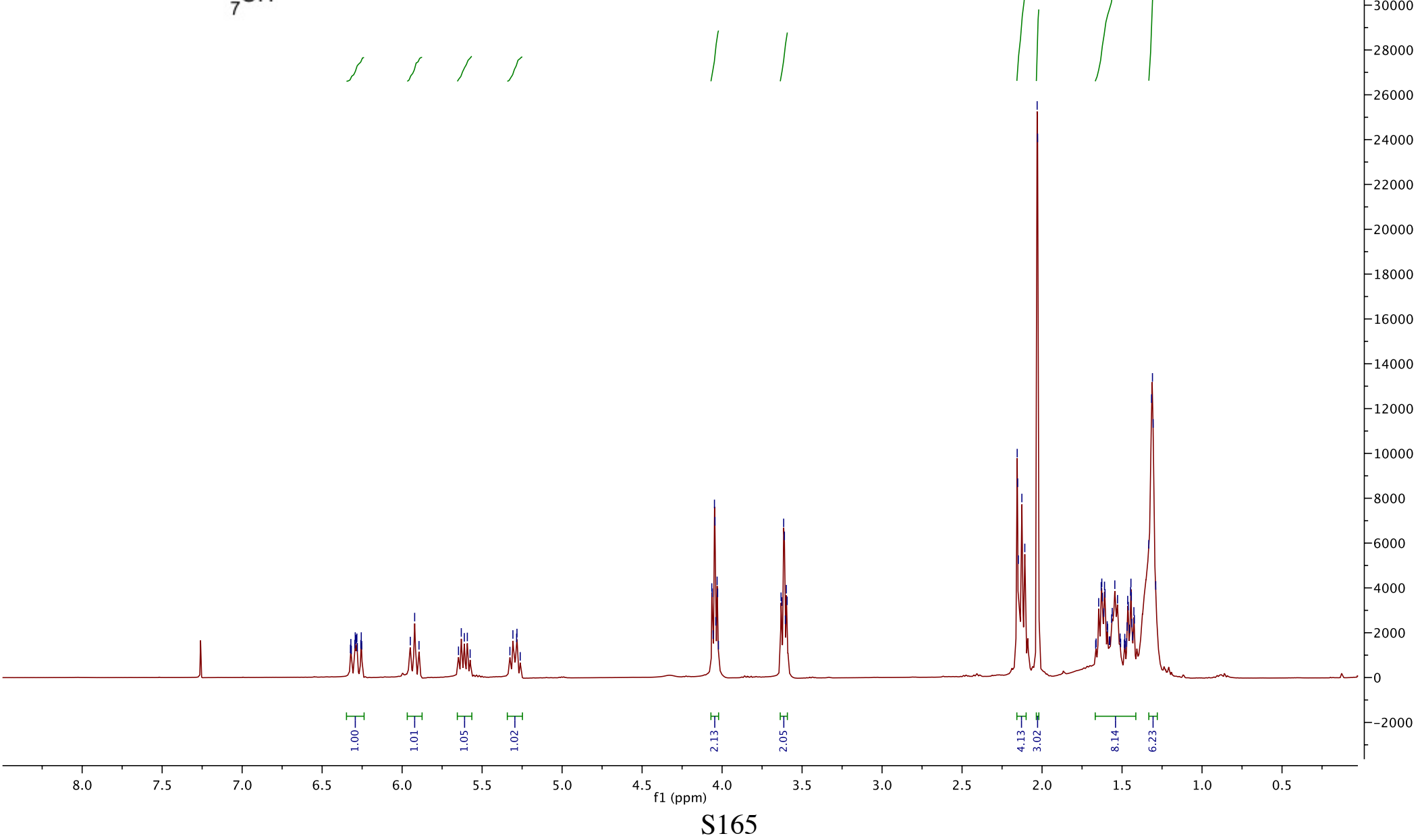


${ }^{13} \mathrm{C}$ NMR (101 MHz, $\mathrm{CDCl}_{3}$ ) spectrum of compound 91.

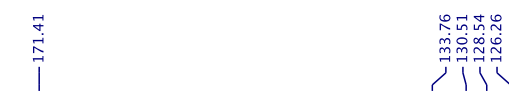



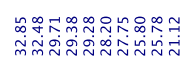

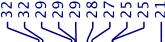

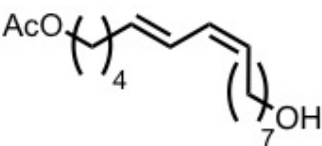
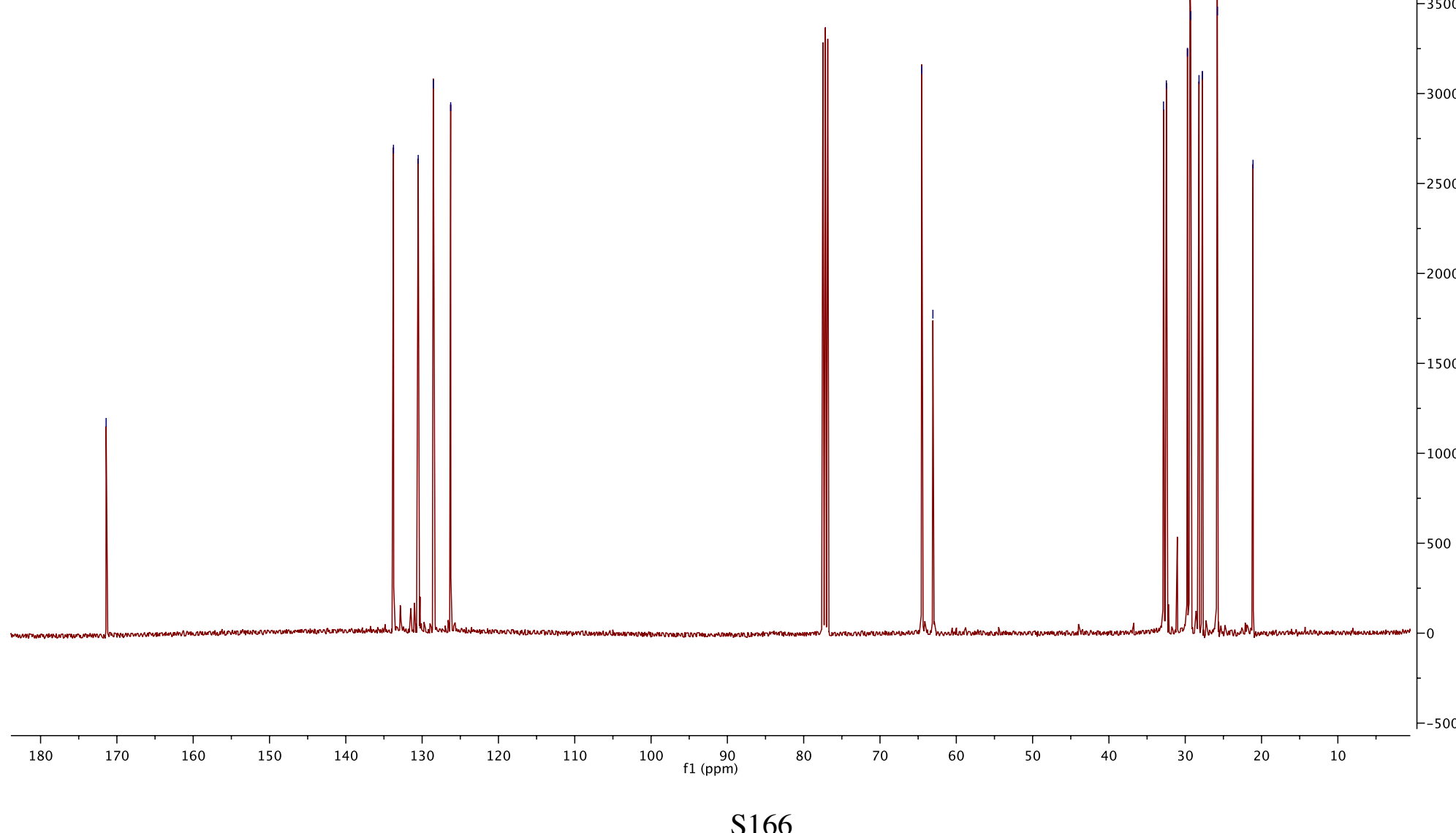
${ }^{1} \mathrm{H}$ NMR (400 MHz, $\mathrm{CDCl}_{3}$ ) spectrum of compound $\mathbf{9 m}$.

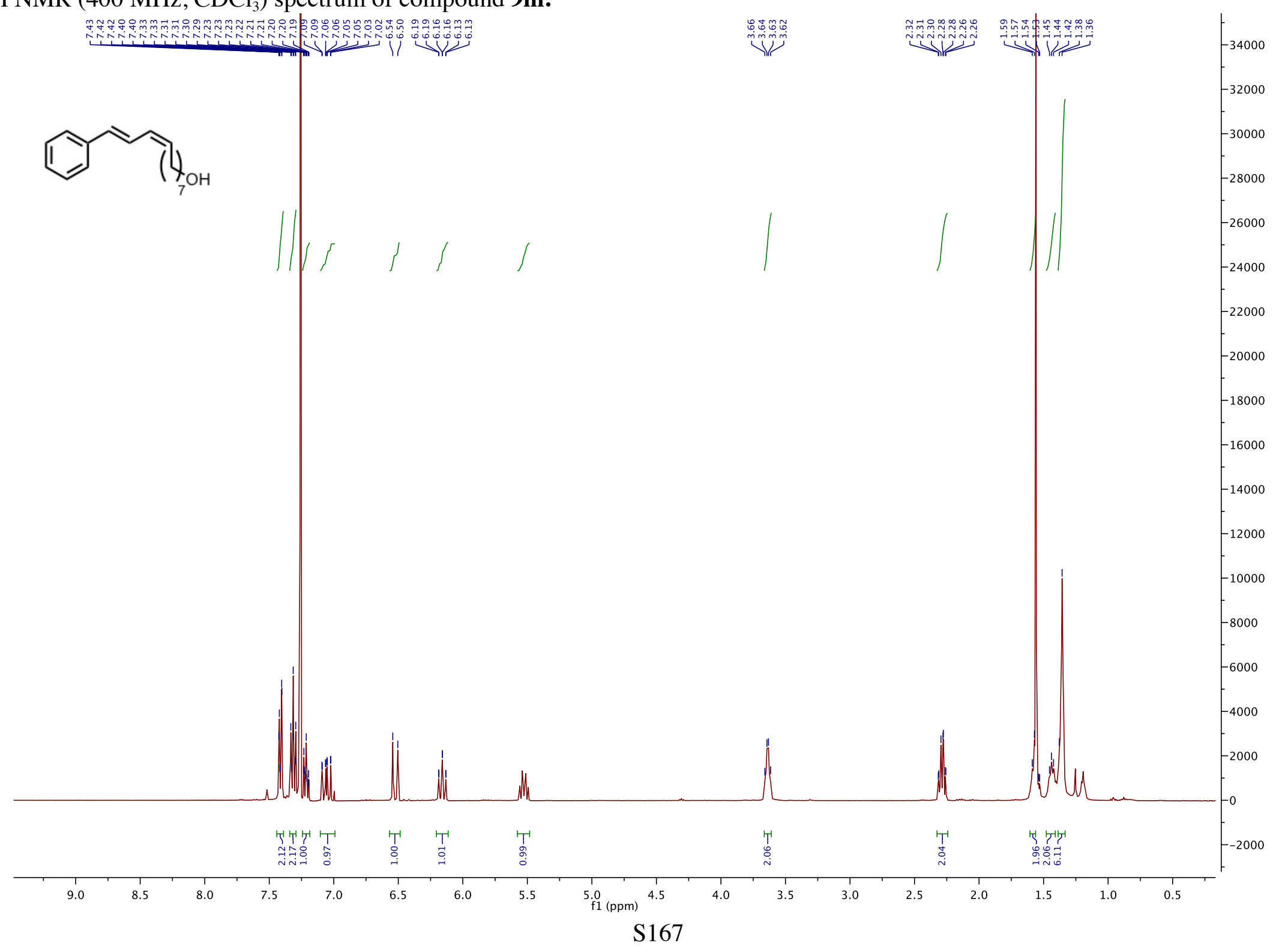


${ }^{13} \mathrm{C}$ NMR (101 MHz, $\left.\mathrm{CDCl}_{3}\right)$ spectrum of compound 9m.

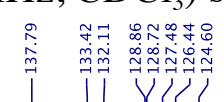

1 (1) $_{7}$
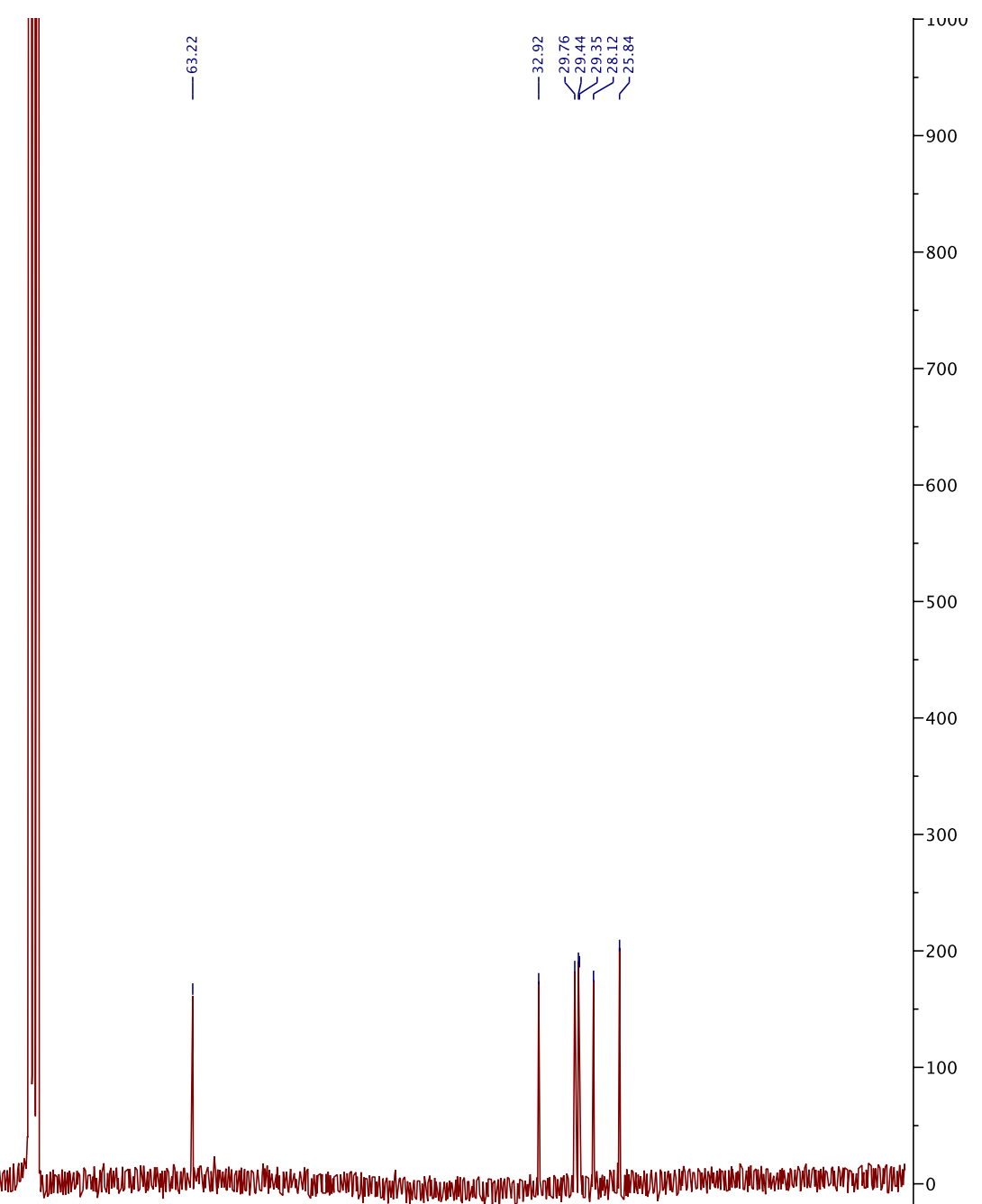

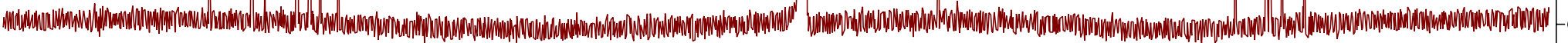

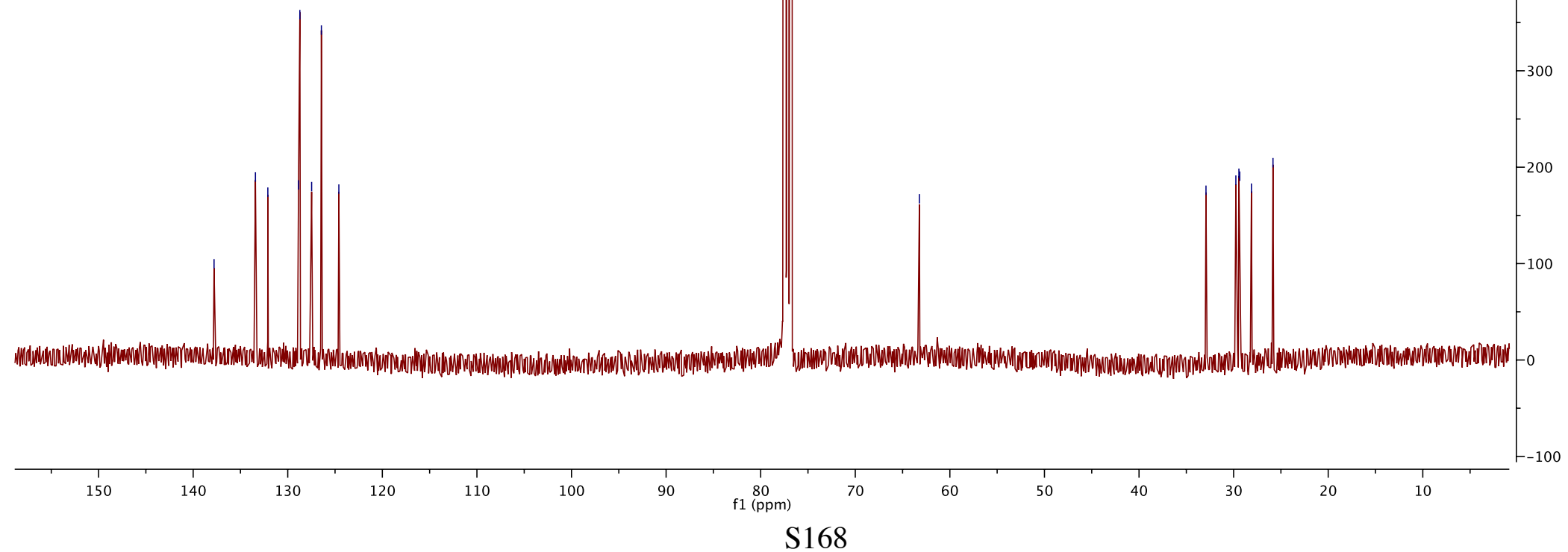


${ }^{1} \mathrm{H}$ NMR (400 MHz, $\mathrm{CDCl}_{3}$ ) spectrum of compound 9n.

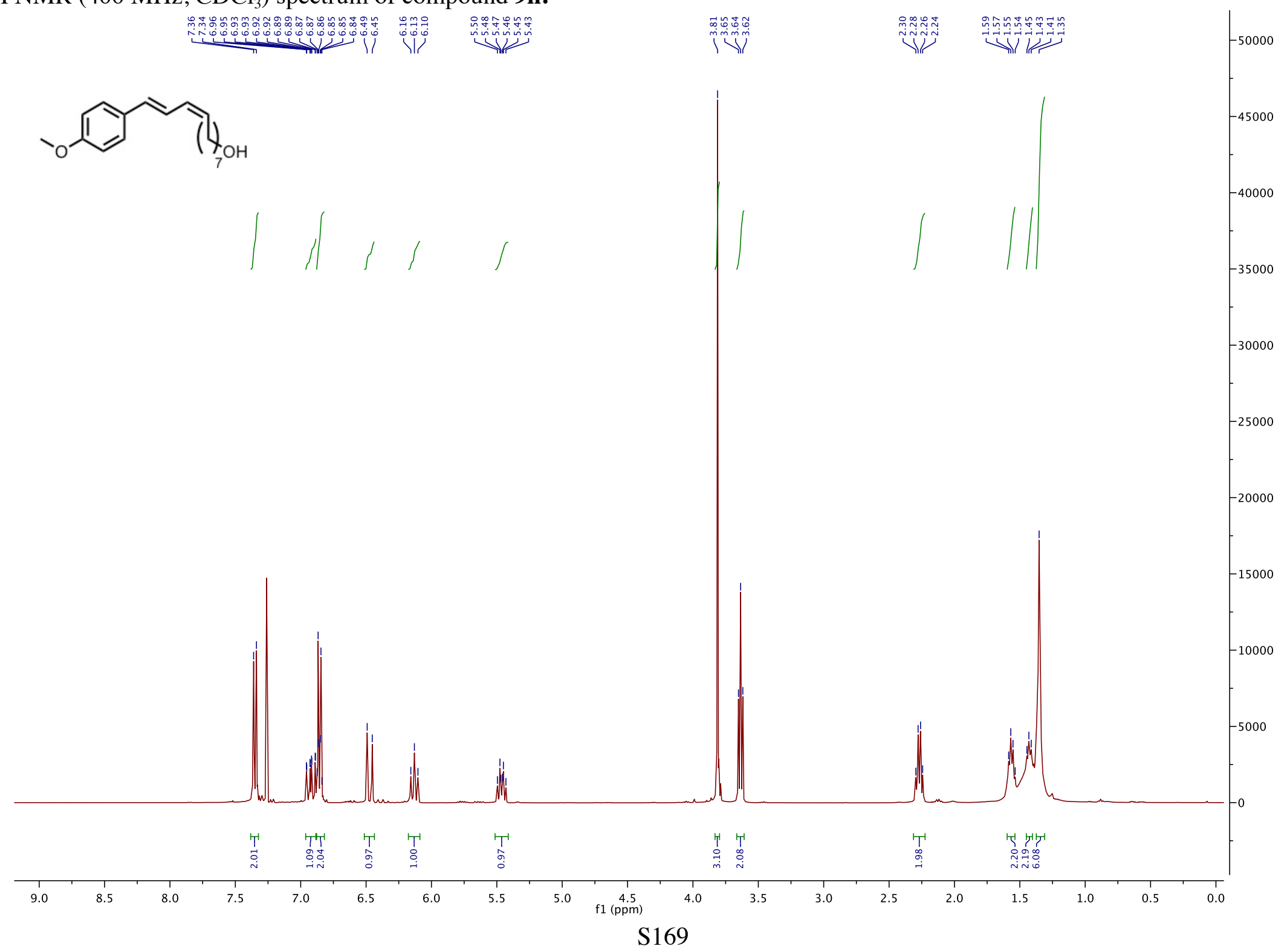


${ }^{13} \mathrm{C}$ NMR (101 MHz, $\mathrm{CDCl}_{3}$ ) spectrum of compound 9n.

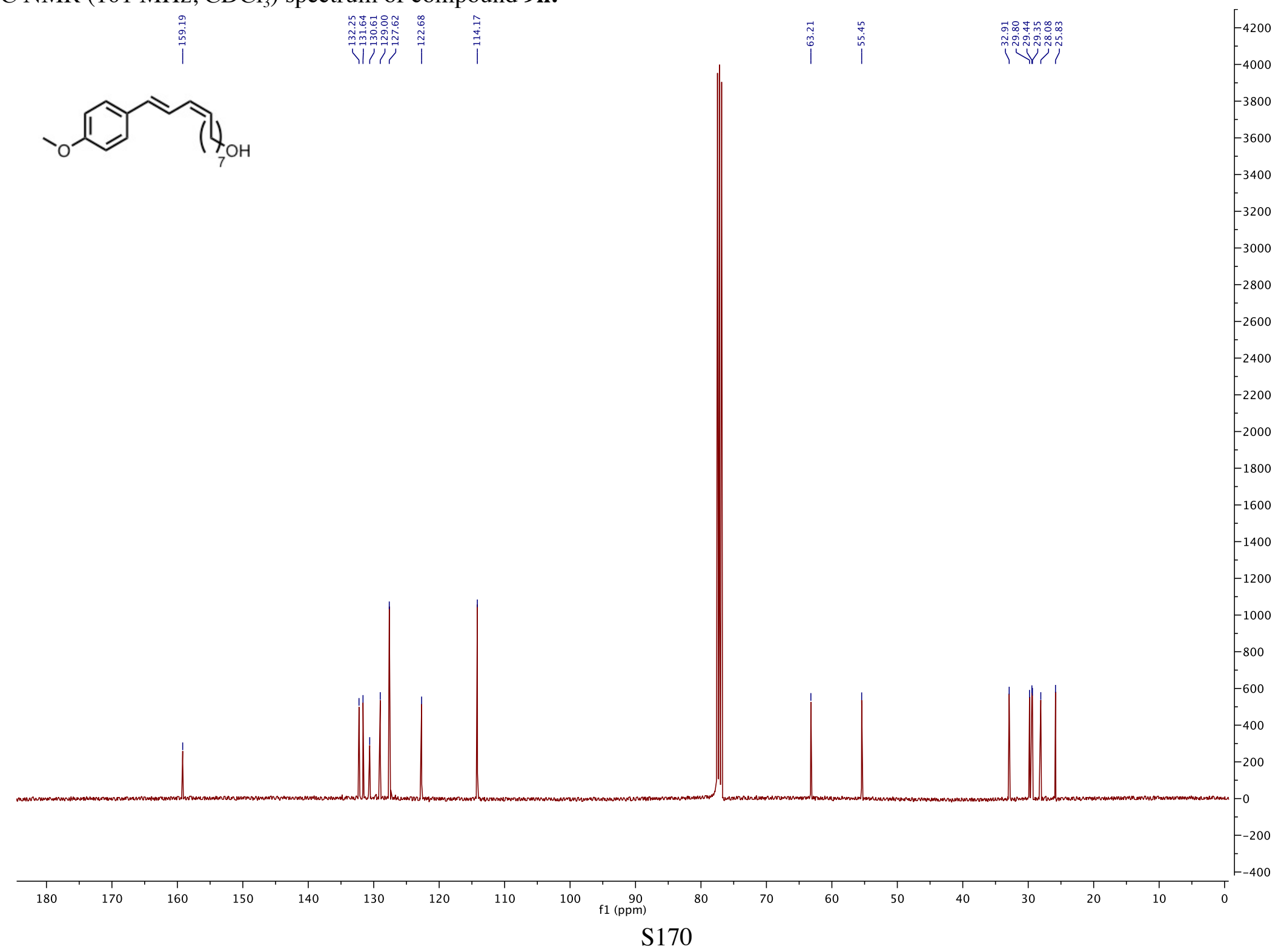


${ }^{1} \mathrm{H}$ NMR (400 MHz, $\mathrm{CDCl}_{3}$ ) spectrum of compound 9o.

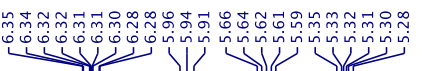<smiles></smiles>
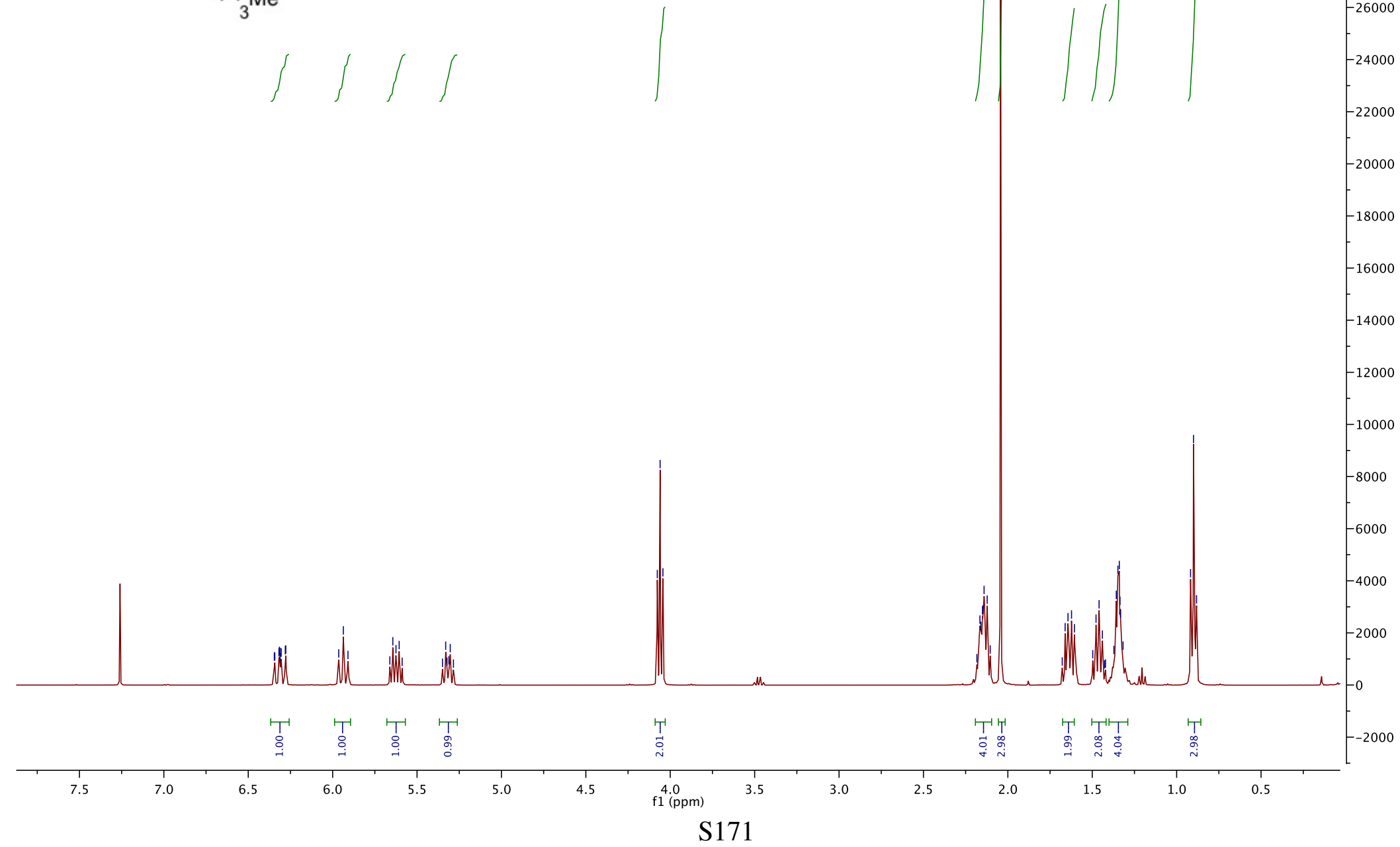
${ }^{13} \mathrm{C}$ NMR (101 MHz, $\mathrm{CDCl}_{3}$ ) spectrum of compound 90.
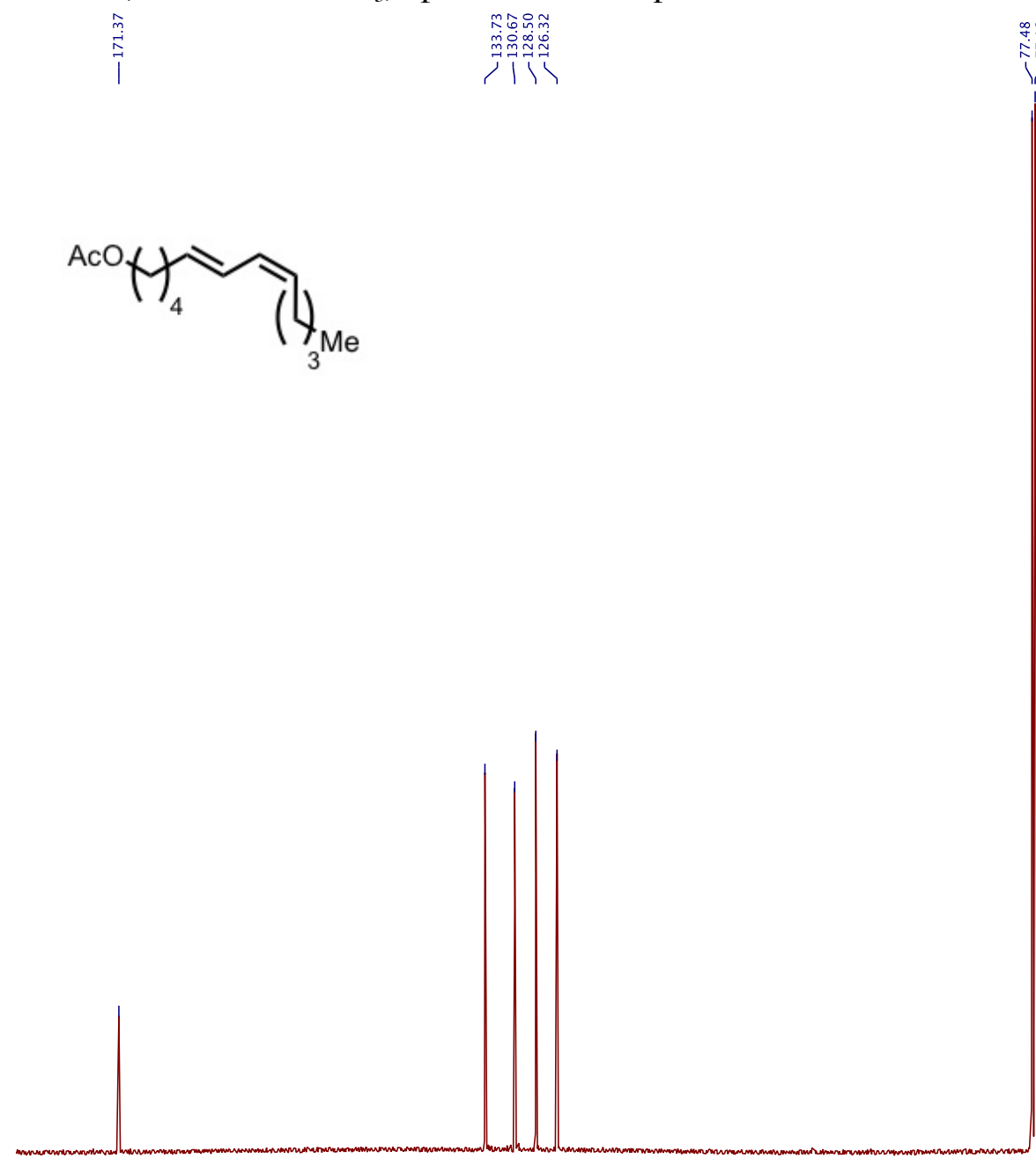

180

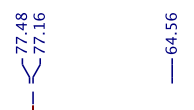

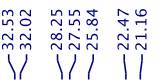

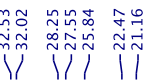

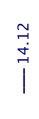

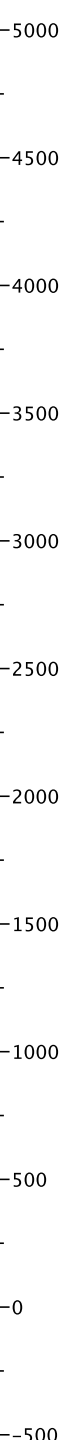

-500

fl $\stackrel{9}{90}$

S172 
${ }^{1} \mathrm{H}$ NMR (400 MHz, $\mathrm{CDCl}_{3}$ ) spectrum of compound 9p.

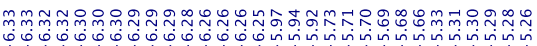

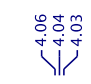<smiles>COC(C)=O</smiles>

${ }_{8} \mathrm{OAC}$

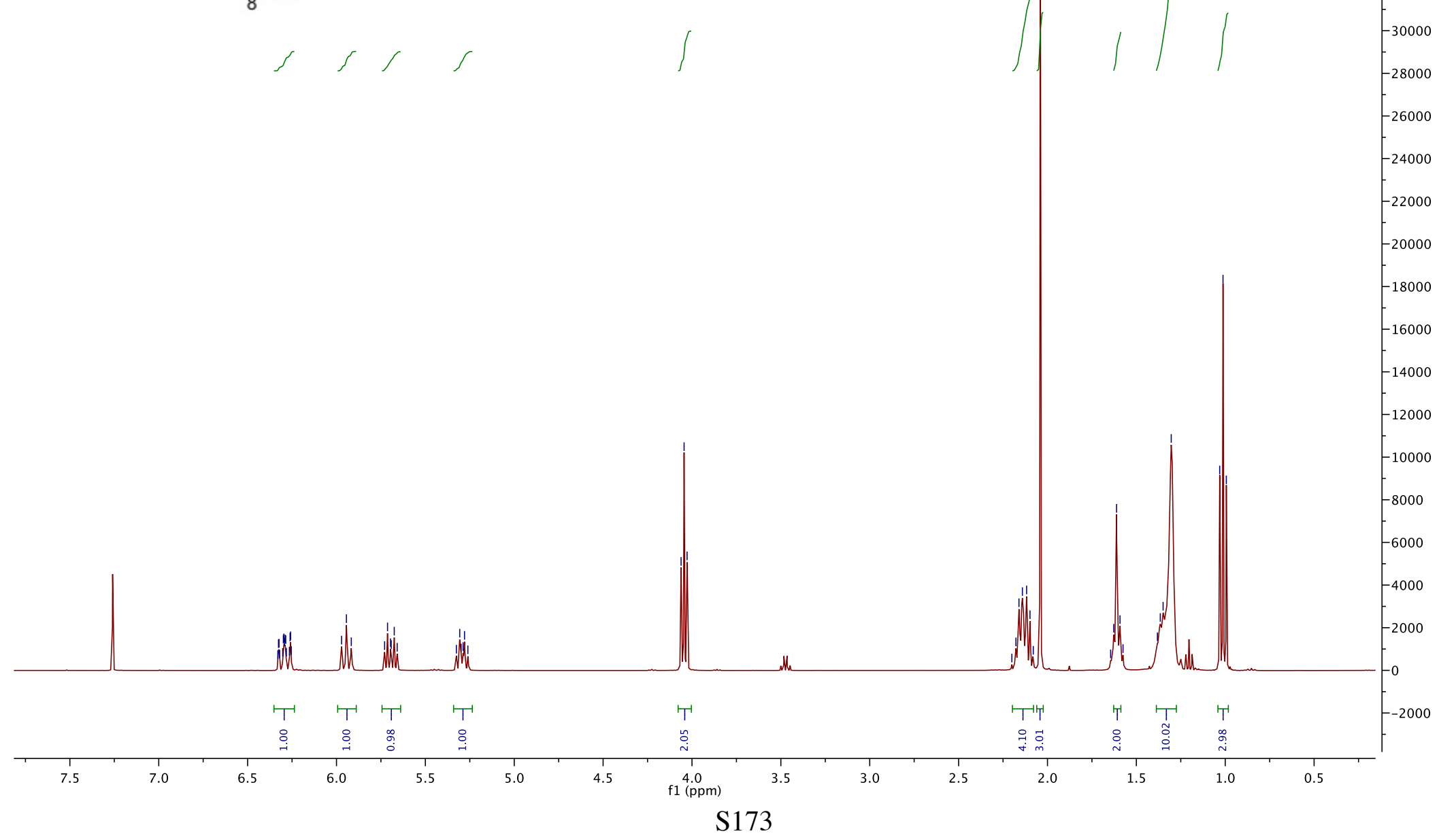


${ }^{13} \mathrm{C}$ NMR (101 MHz, $\mathrm{CDCl}_{3}$ ) spectrum of compound 9p.

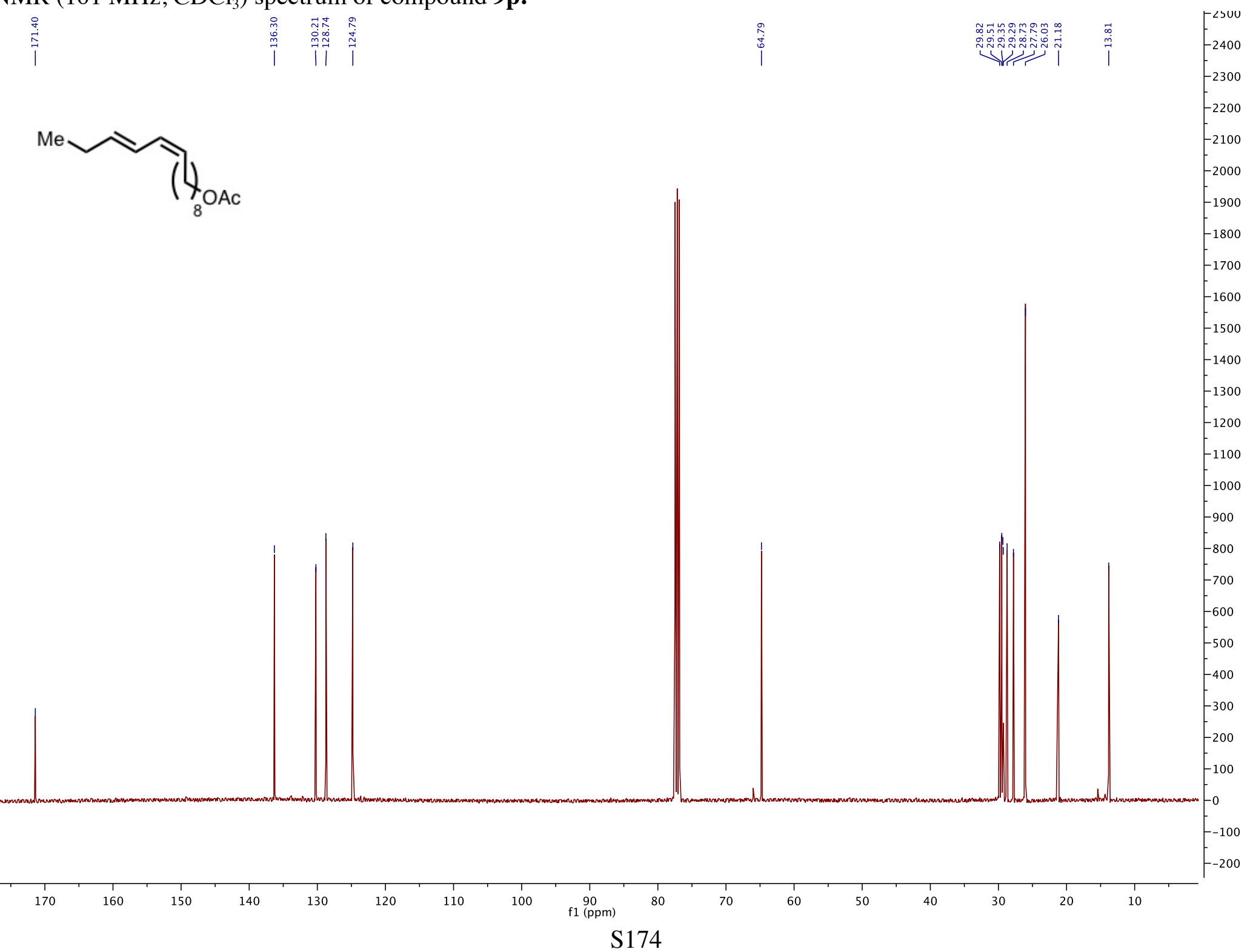


${ }^{1} \mathrm{H}$ NMR $\left(500 \mathrm{MHz}, \mathrm{CDCl}_{3}\right.$ ) spectrum of compound 28a.

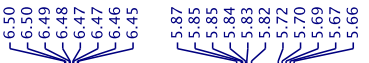
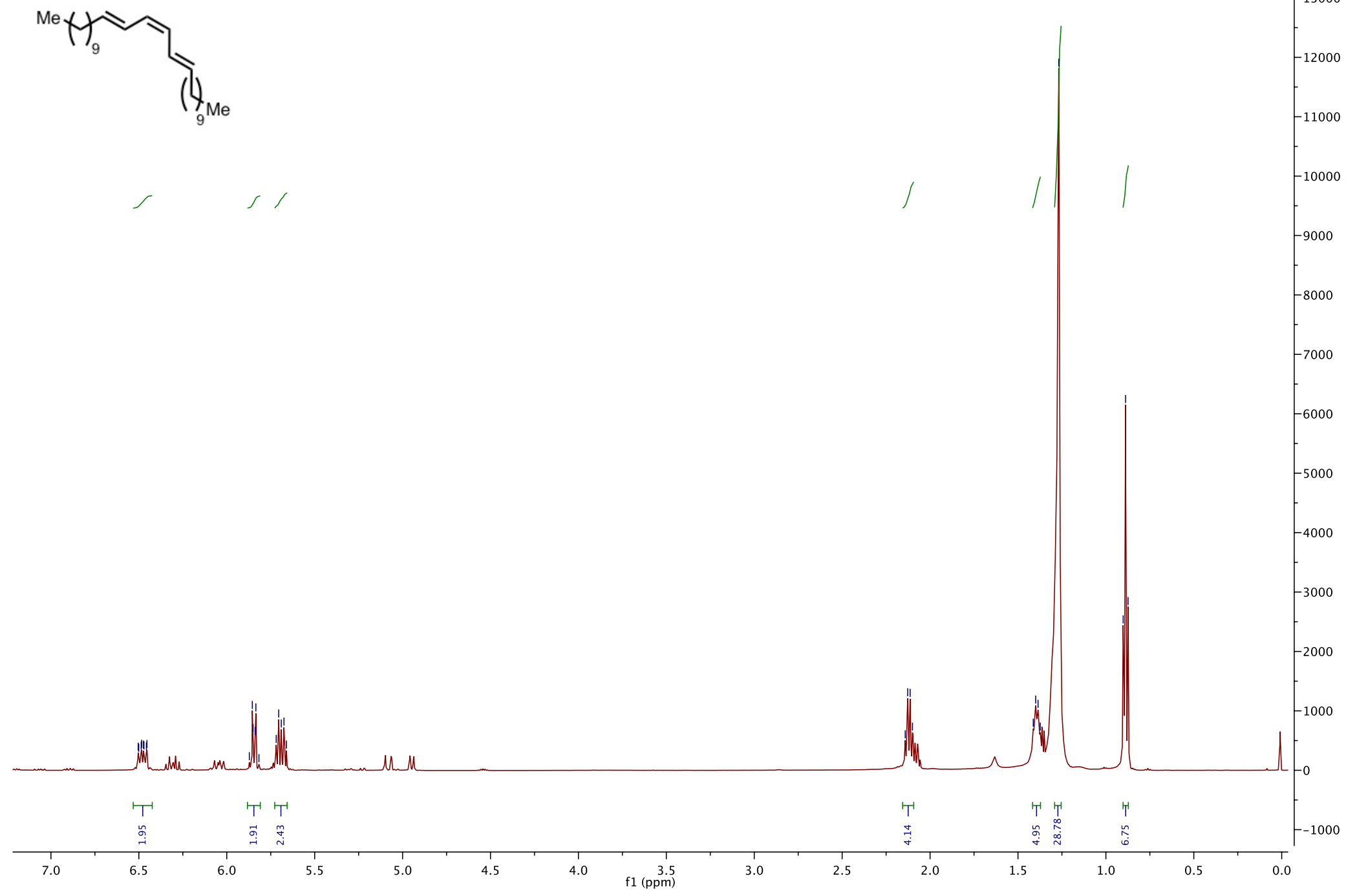
${ }^{1} \mathrm{H}$ NMR (400 MHz, $\mathrm{CDCl}_{3}$ ) spectrum of compound $28 \mathbf{b}$.

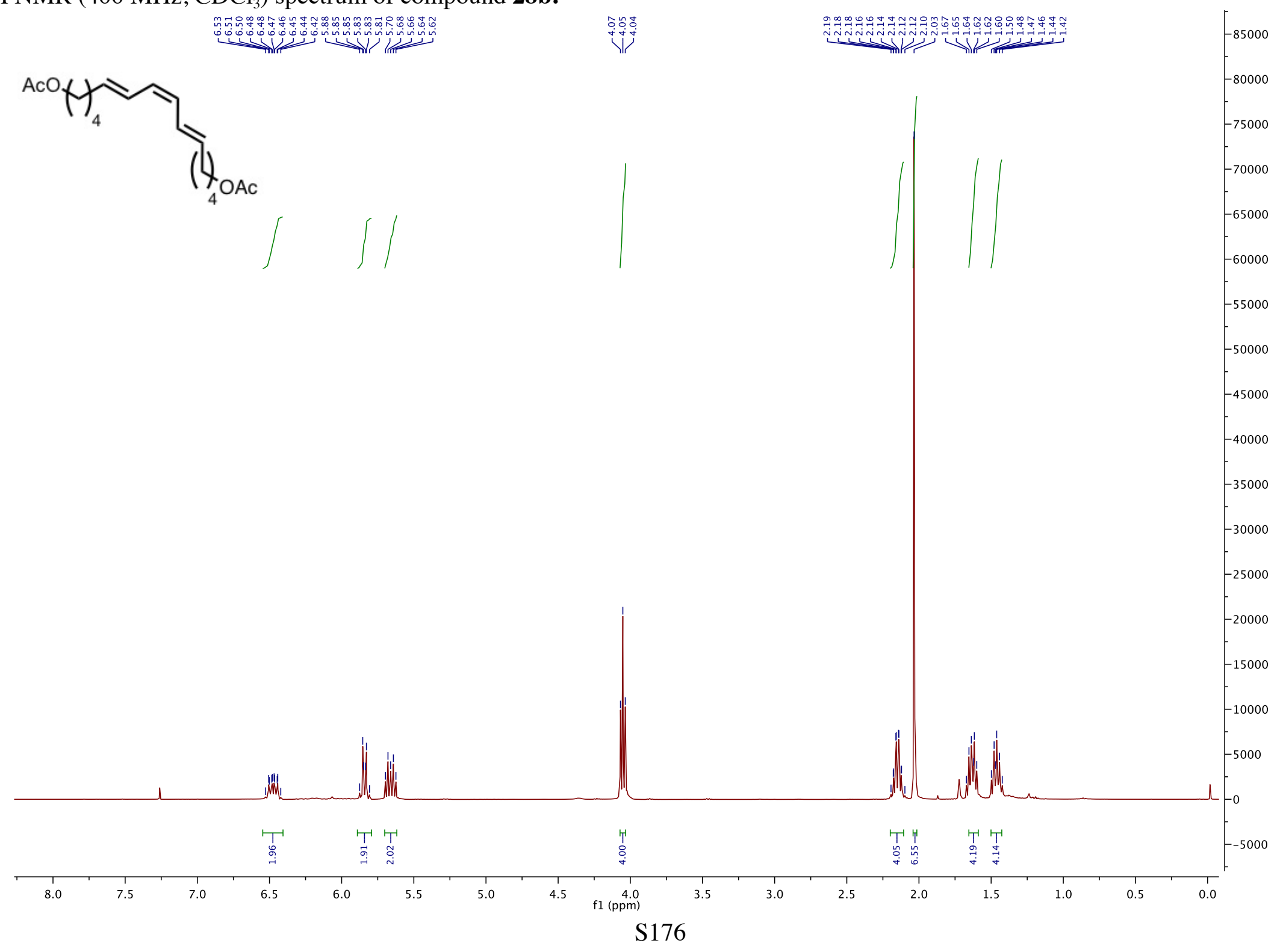


${ }^{13} \mathrm{C}$ NMR (101 MHz, $\mathrm{CDCl}_{3}$ ) spectrum of compound $\mathbf{2 8 b}$.

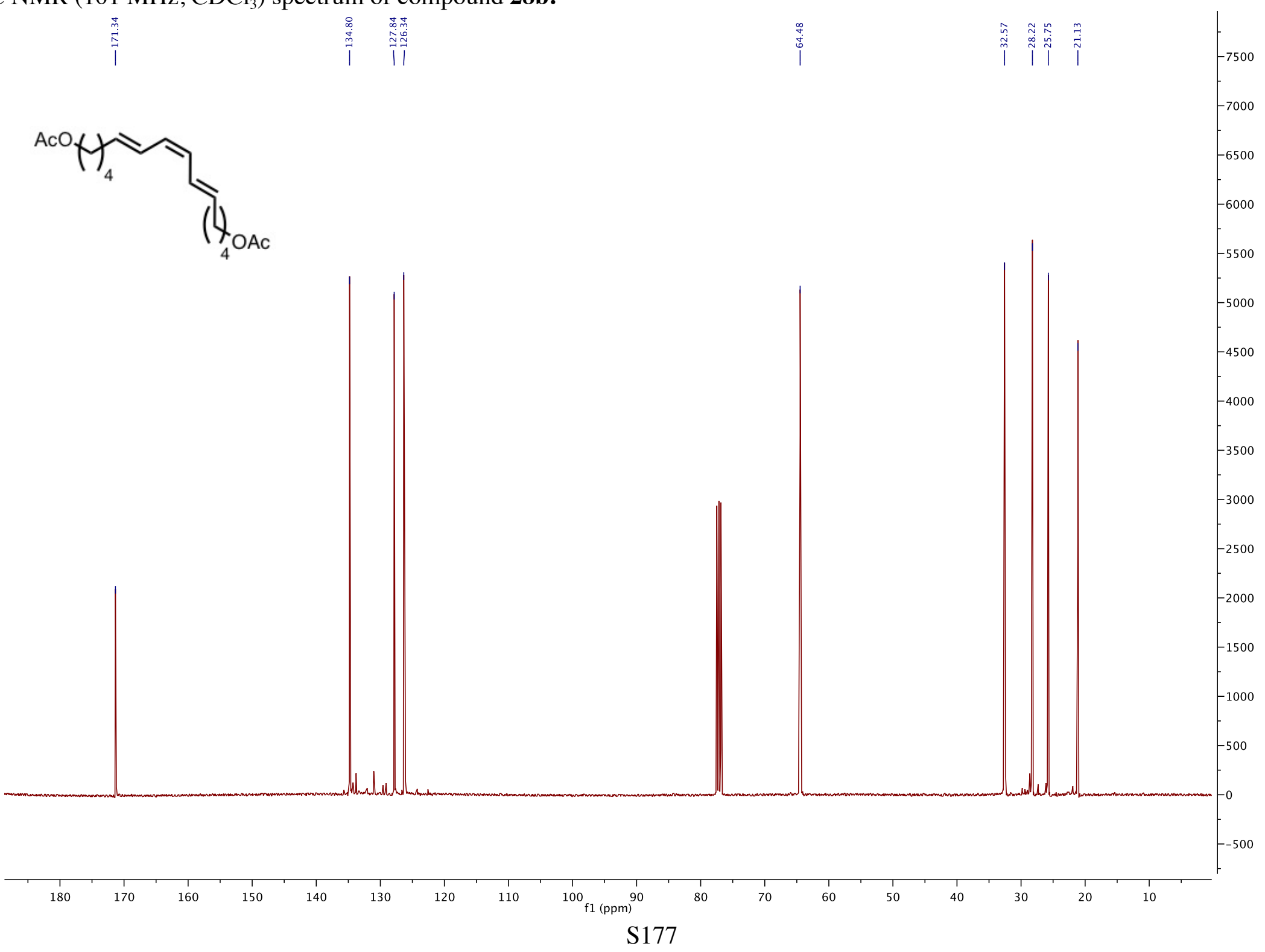


${ }^{1} \mathrm{H}$ NMR (400 MHz, $\mathrm{CDCl}_{3}$ ) spectrum of compound 28c.

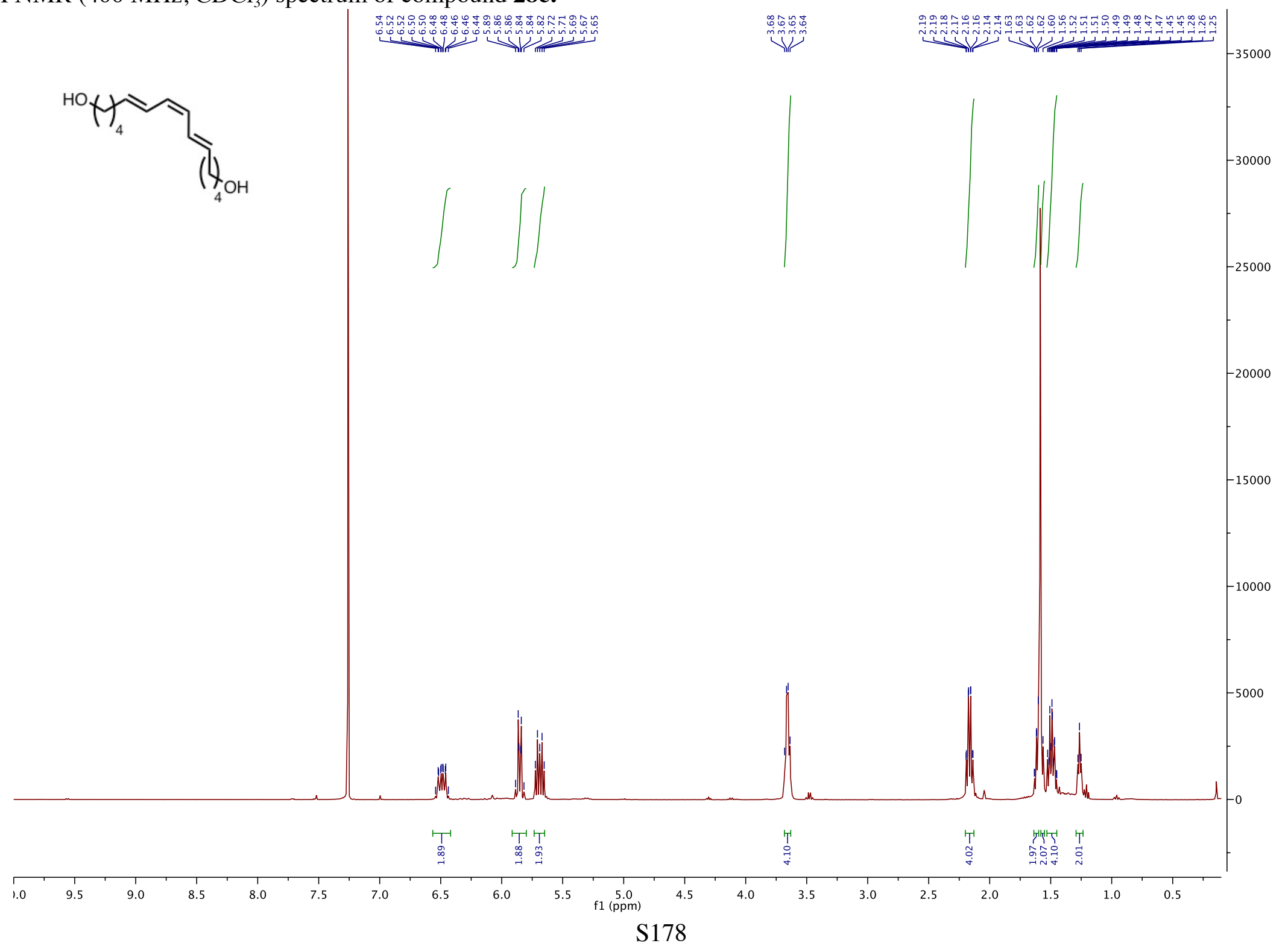


${ }^{13} \mathrm{C}$ NMR (101 MHz, $\mathrm{CDCl}_{3}$ ) spectrum of compound 28c.

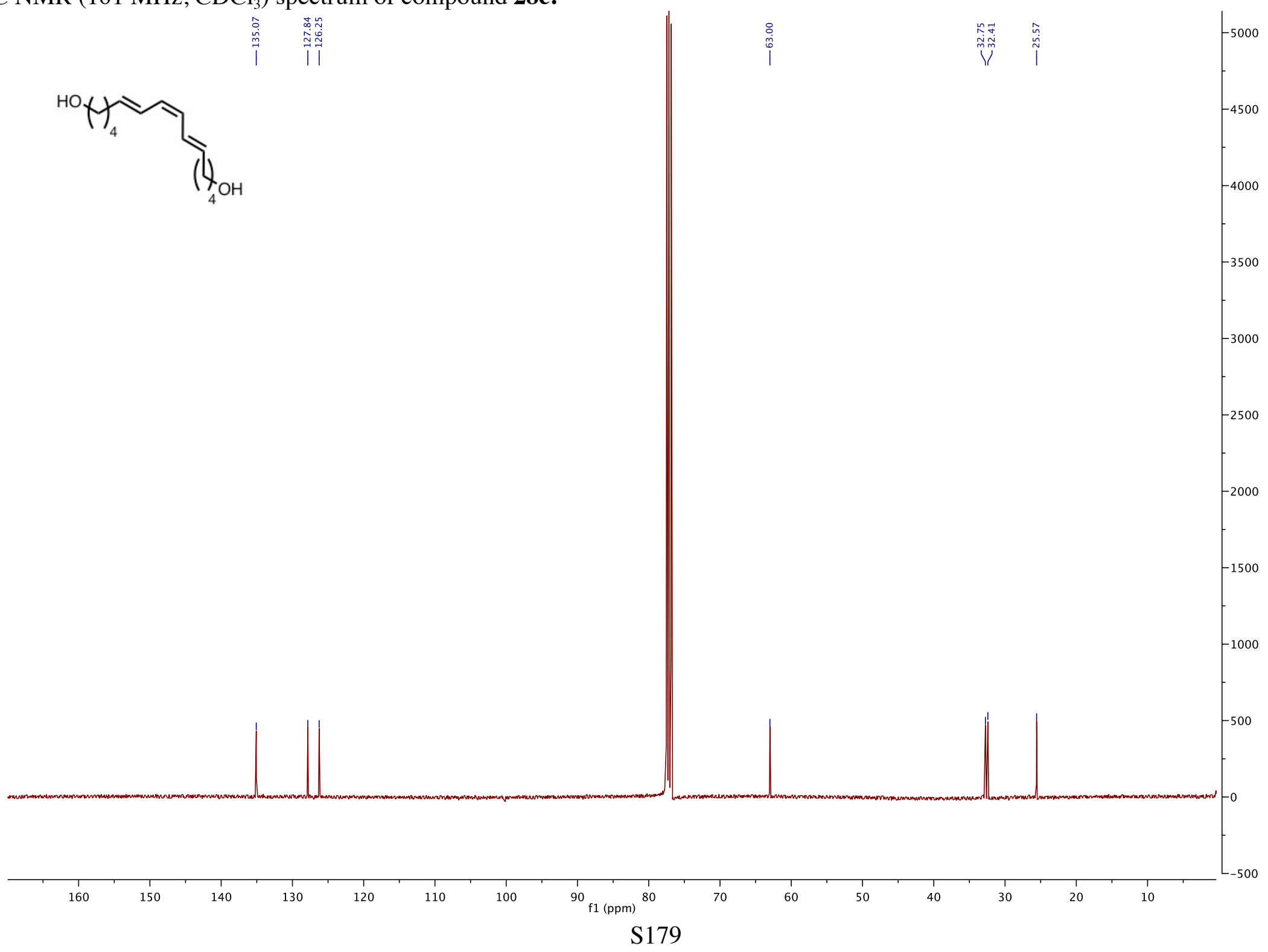


${ }^{1} \mathrm{H} \mathrm{NMR}\left(500 \mathrm{MHz}, \mathrm{CDCl}_{3}\right)$ spectrum of compound 30a.

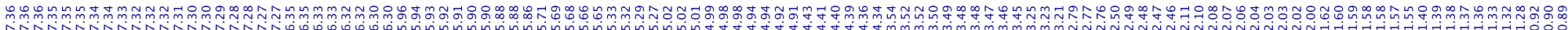

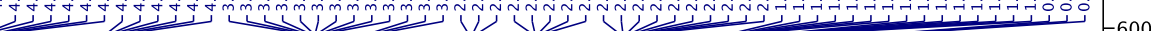

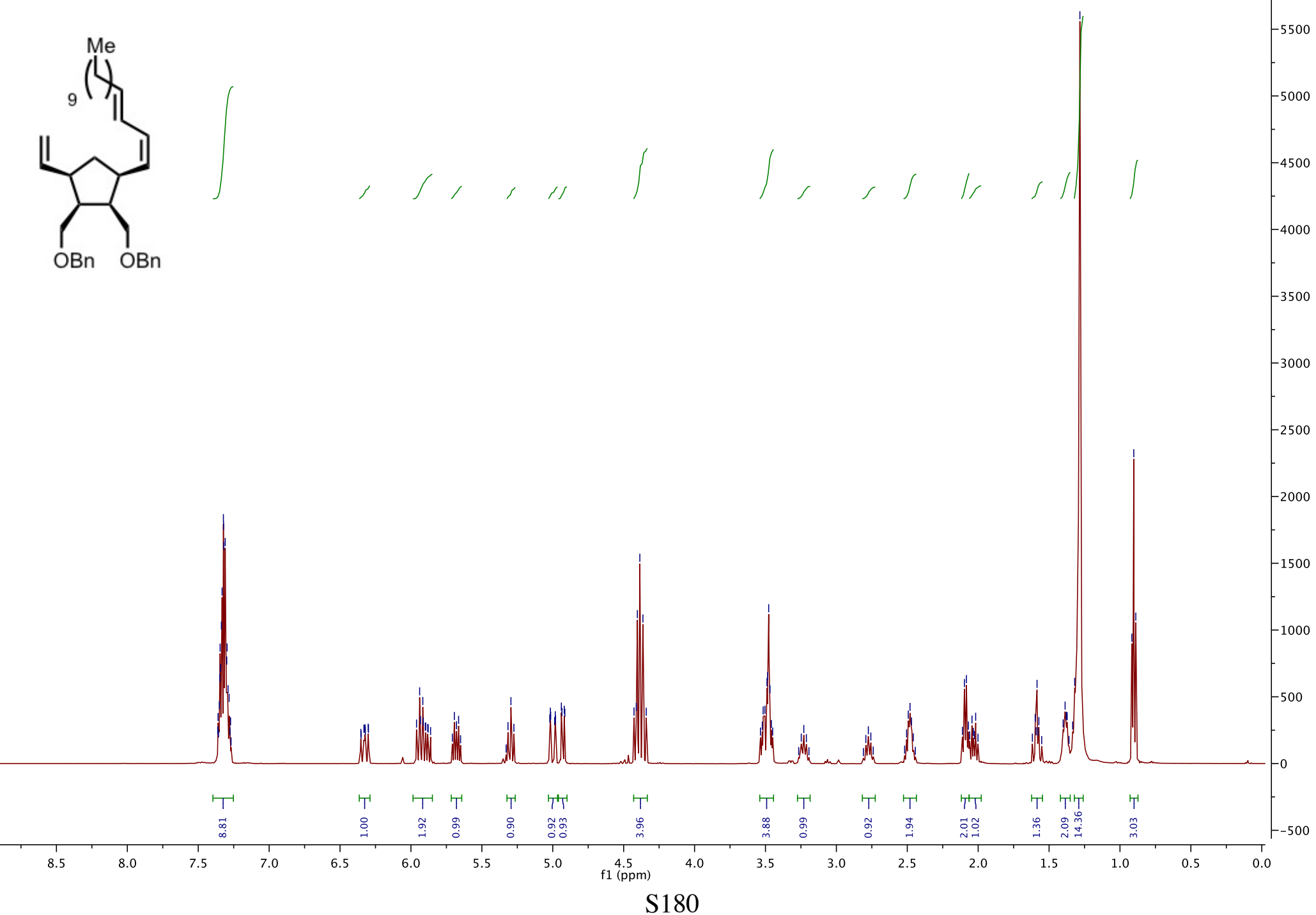


${ }^{13} \mathrm{C}$ NMR (126 MHz, $\mathrm{CDCl}_{3}$ ) spectrum of compound 30a.

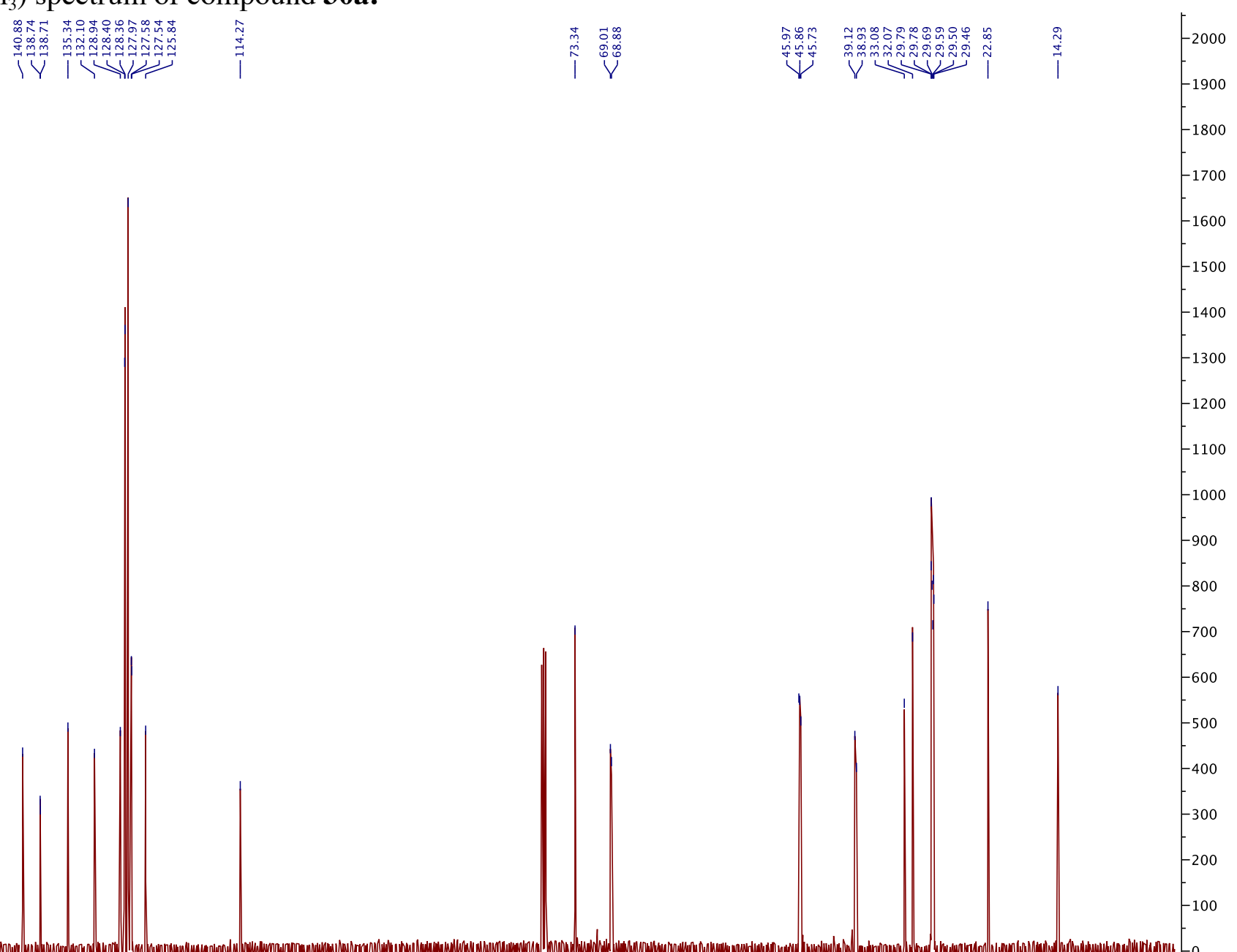

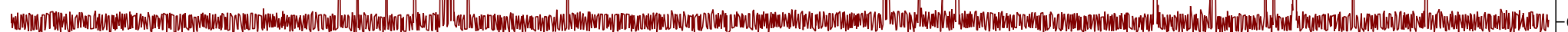
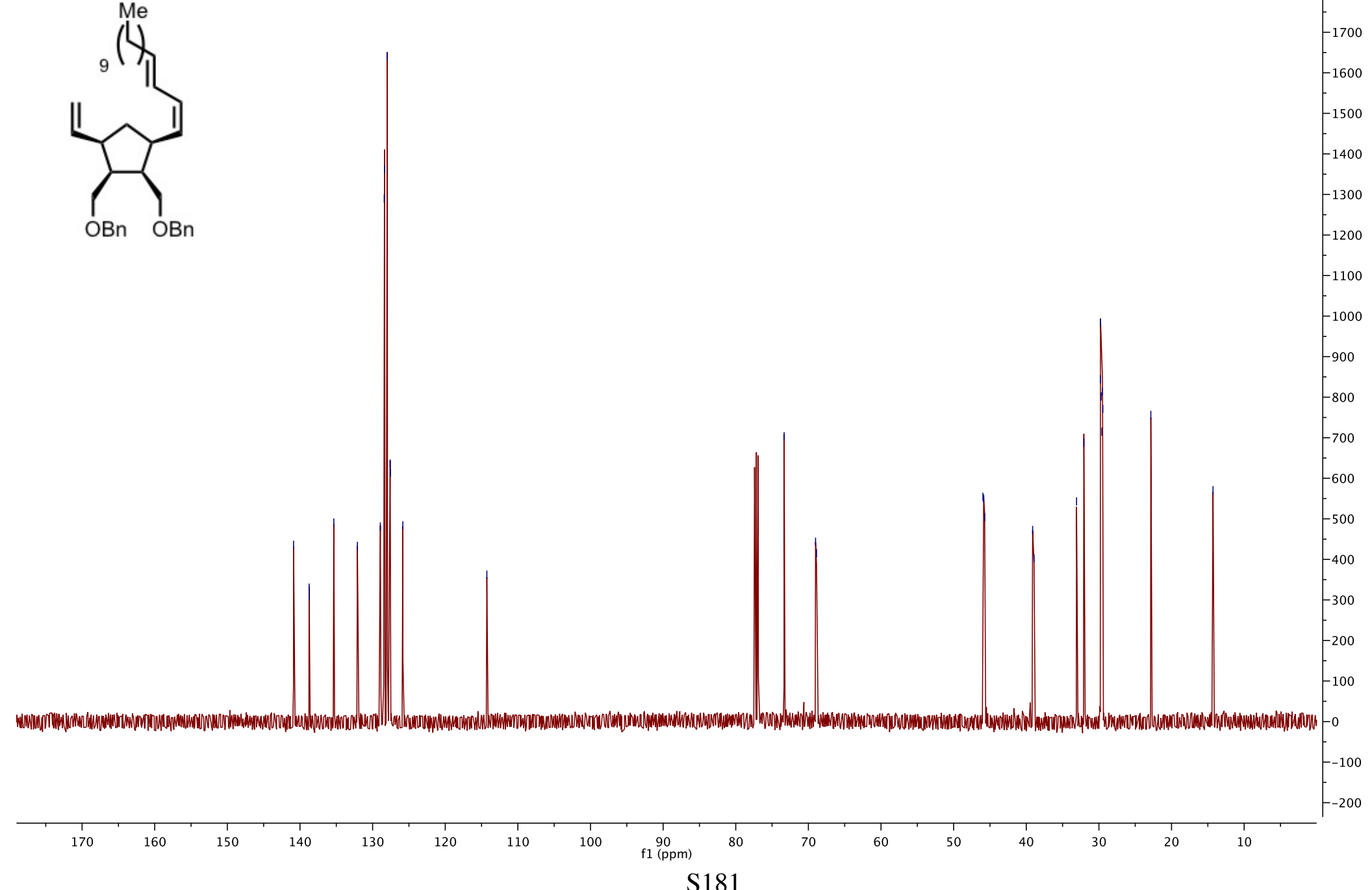
gCOSY spectrum of compound 30a.

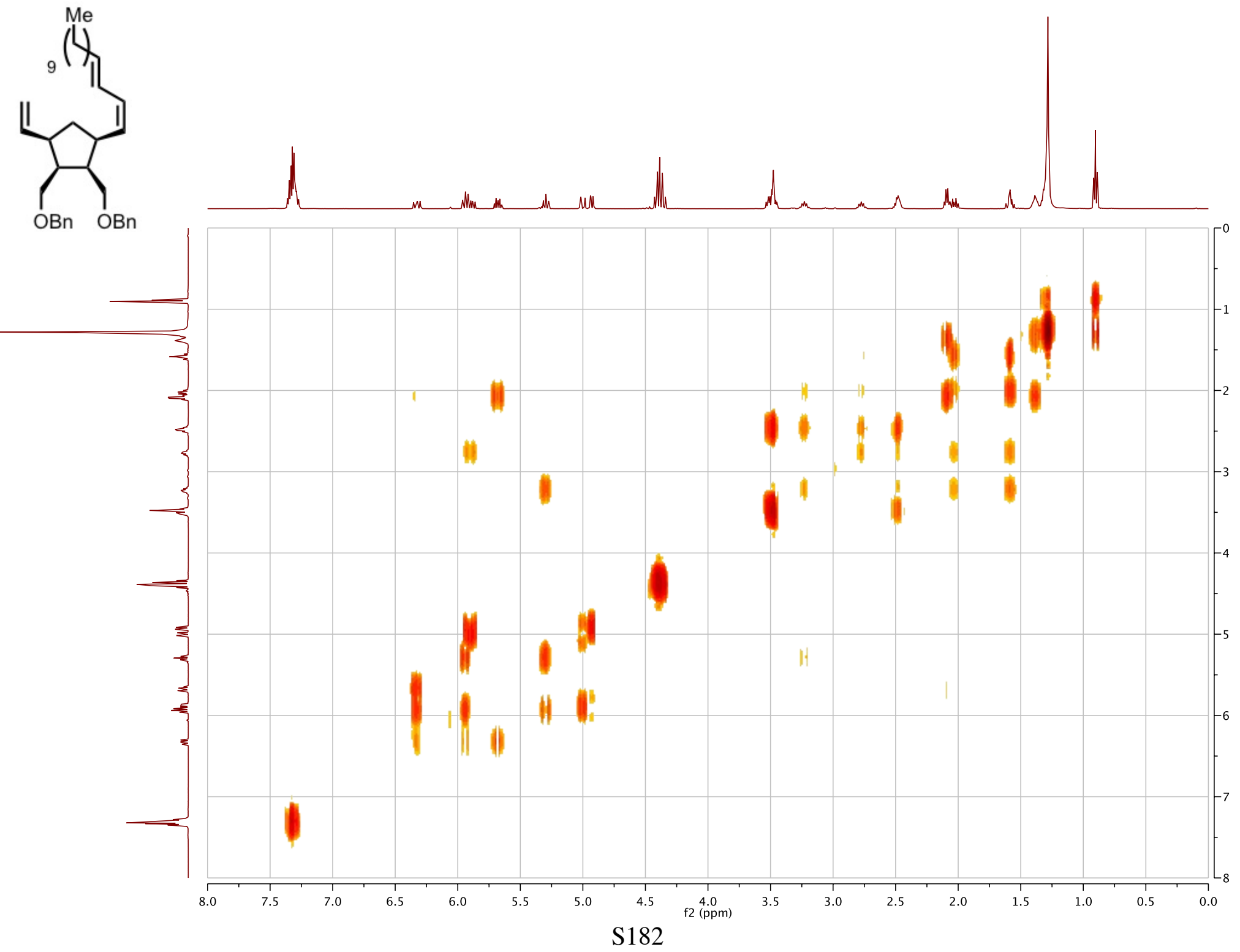


HSQC spectrum of compound 30a.

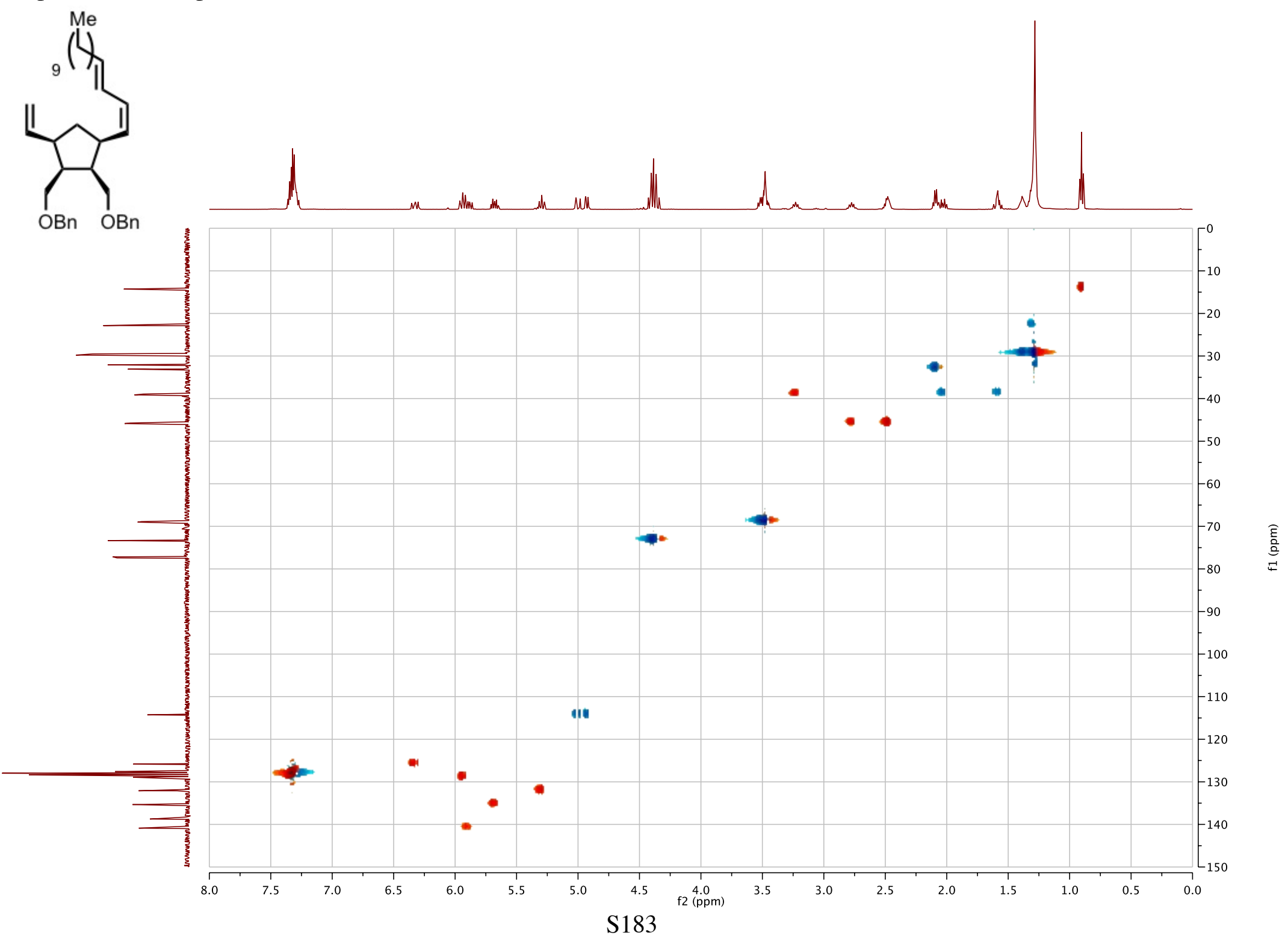


${ }^{1} \mathrm{H}$ NMR (500 MHz, $\mathrm{CDCl}_{3}$ ) spectrum of compound $\mathbf{3 0 b}$.

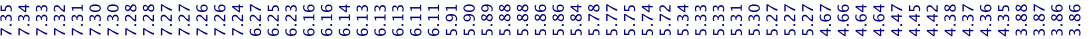
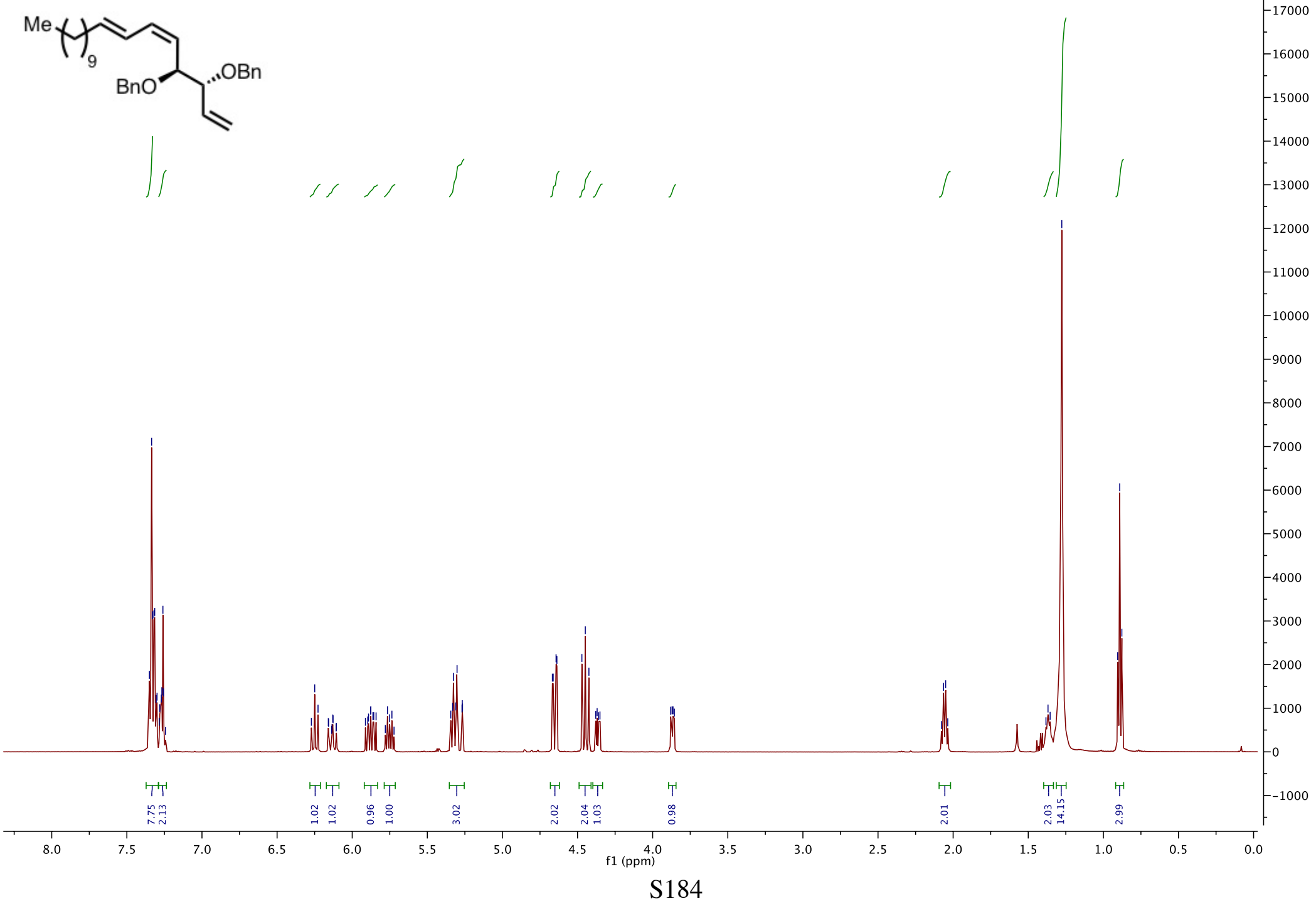
${ }^{13} \mathrm{C}$ NMR (126 MHz, $\mathrm{CDCl}_{3}$ ) spectrum of compound $\mathbf{3 0 b}$.

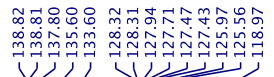<smiles>C=C[C@@H](OBr)C(/C=C\C=C/C(C)(C)C)OBr</smiles>

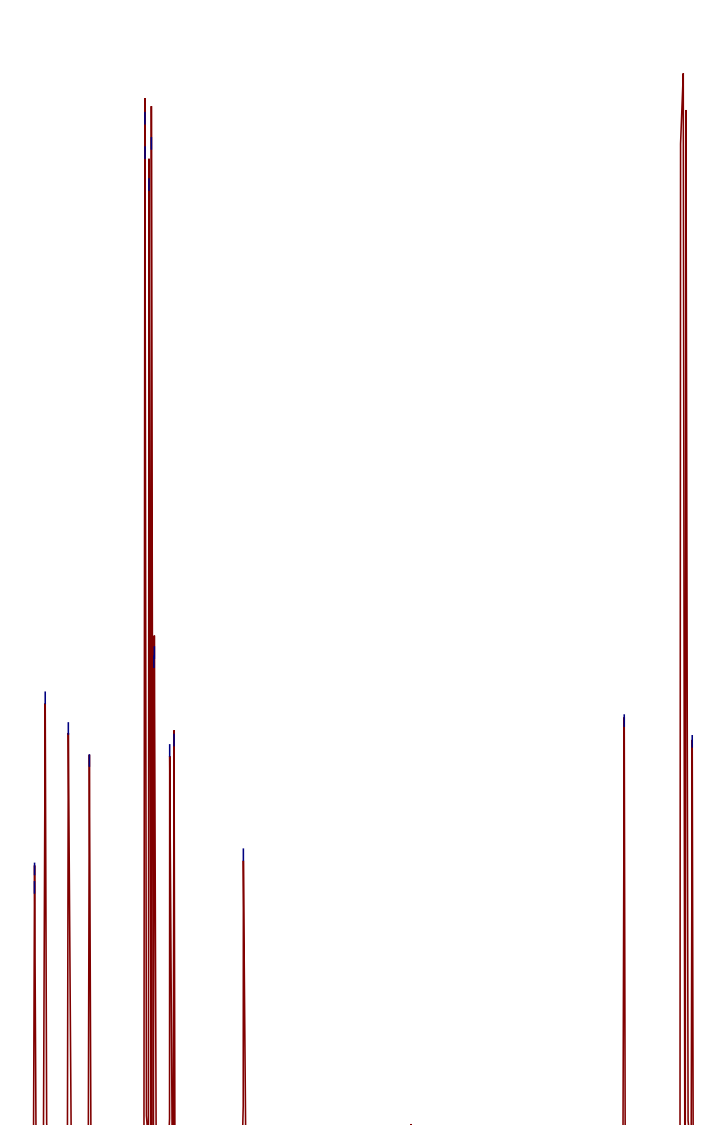

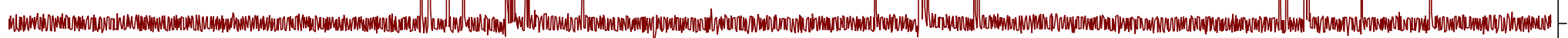

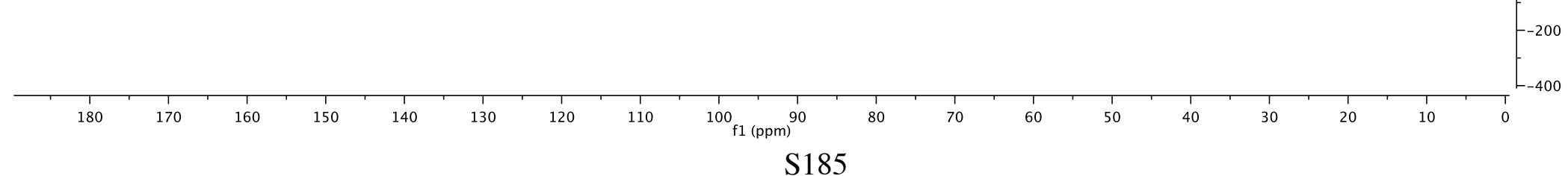


gCOSY spectrum of compound $\mathbf{3 0 b}$.

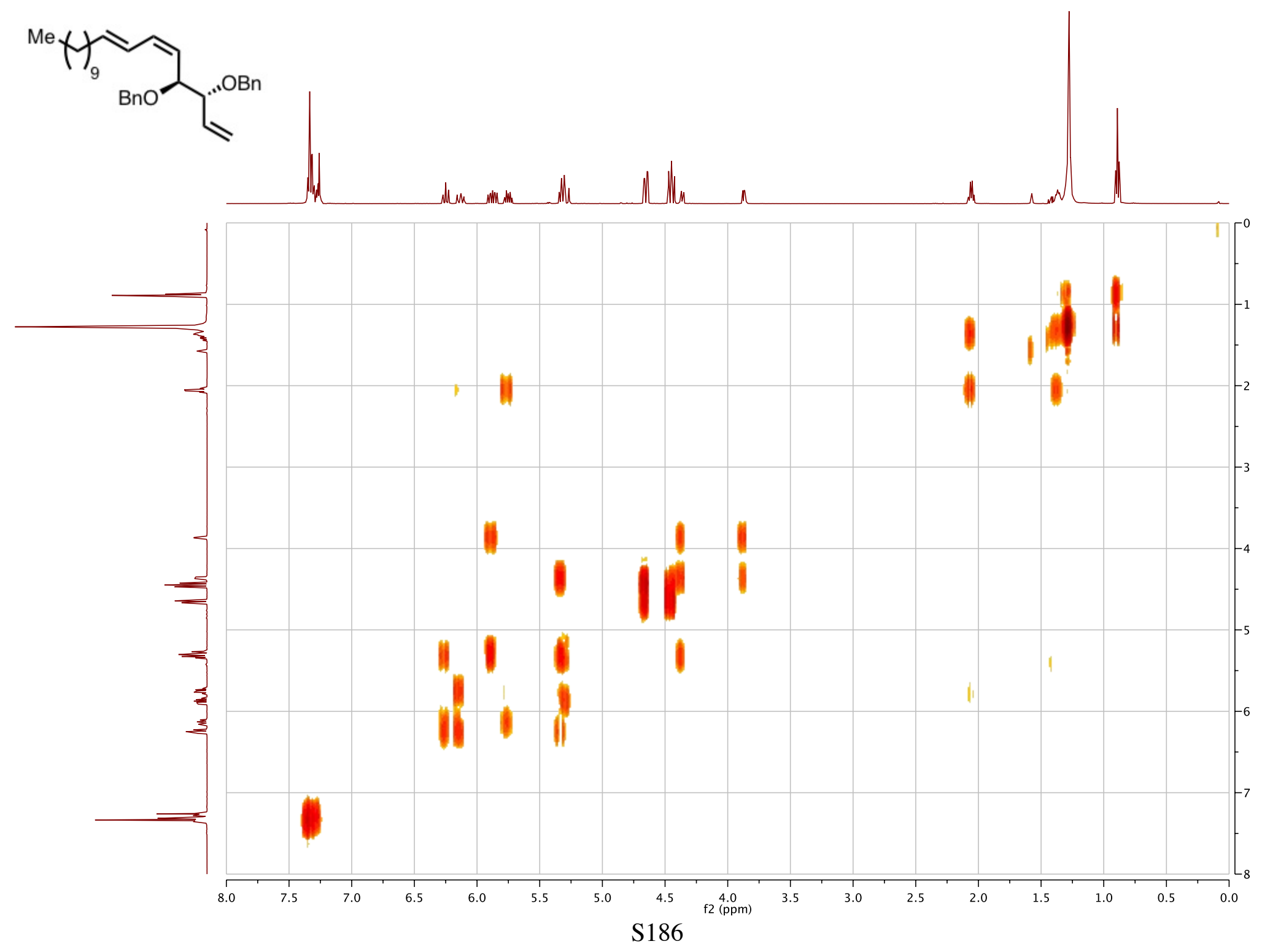


HSQC spectrum of compound $\mathbf{3 0 b}$.

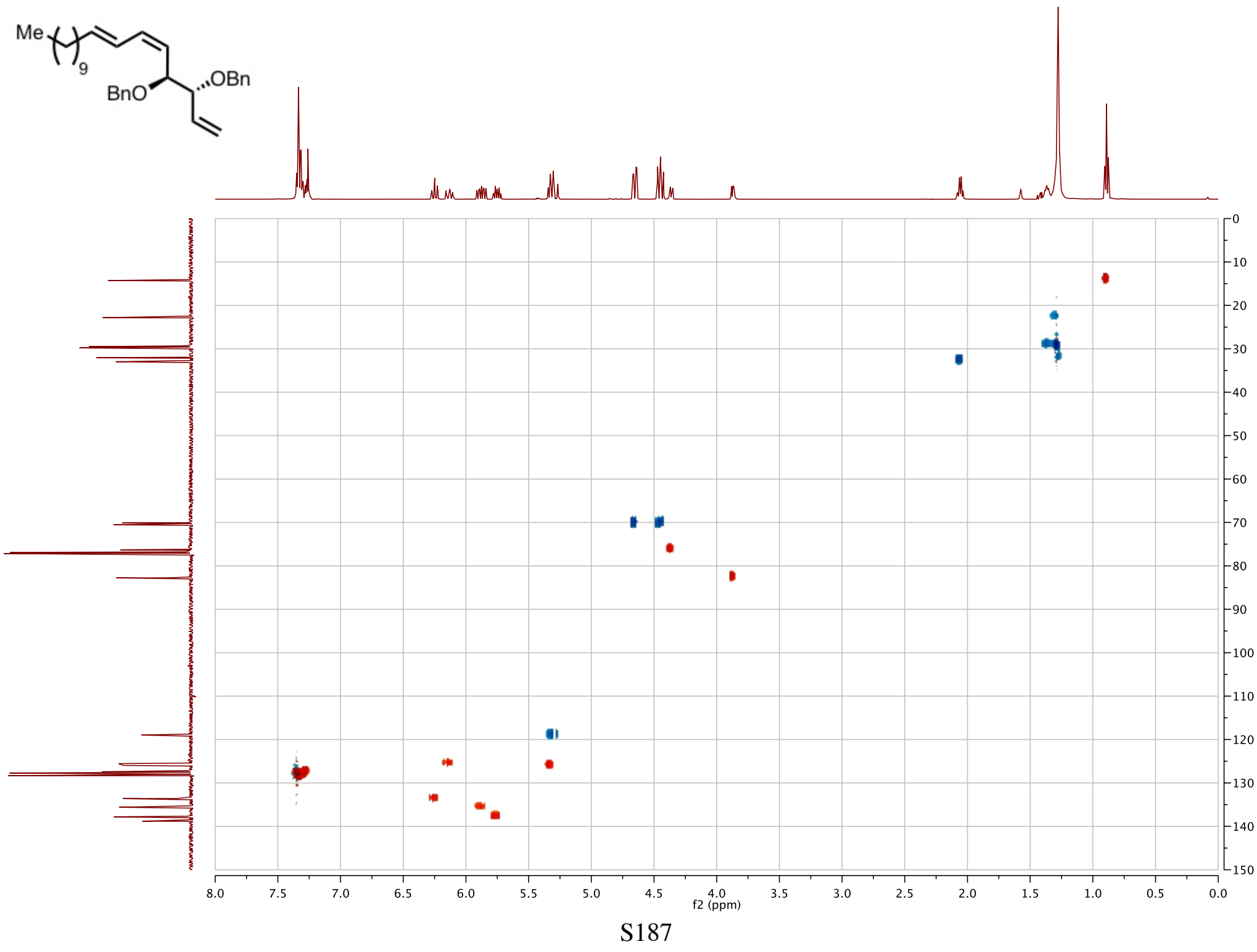

